
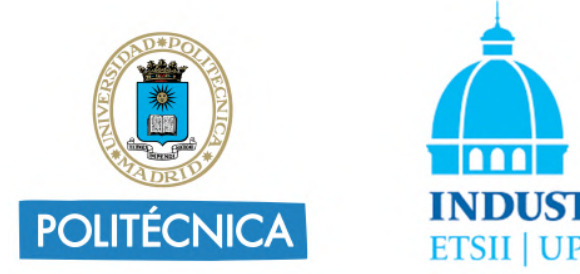

INDUSTRIALES

ETSII | UPM

Universidad Politécnica de Madrid

Escuela Técnica Superior de Ingenieros Industriales

\title{
DEEP AND REINFORCEMENT LEARNING IN Perception AND CONTROL FOR Autonomous AERIAL RoBots
}

Ph.D. Thesis

\author{
Alejandro Rodríguez Ramos \\ M.Sc. in Telecommunication Engineering
}

2020 



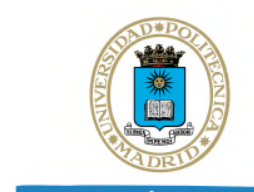

\title{
POLITÉCNICA
}

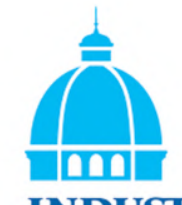

INDUSTRIALES

ETSII | UPM

Departamento de Automática, Ingeniería Electrónica e Informática Industrial

Escuela Técnica Superior de Ingenieros Industriales Universidad Politécnica de Madrid

\section{DEEP AND REINFORCEMENT LEARNING IN PERCEPTION AND CONTROL FOR Autonomous Aerial Robots}

\author{
A thesis submitted for the degree of \\ Doctor of Philosophy in Automation and Robotics
}

Author:

Alejandro Rodríguez Ramos

M.Sc. in Telecommunication Engineering

Director:

Pascual Campoy Cervera

Prof. Dr. in Automation and Robotics 

Título:

\title{
Deep and Reinforcement Learning in Perception and Control for Autonomous Aerial Robots
}

Autor:

Alejandro Rodríguez Ramos

M.Sc. in Telecommunication Engineering

B.Sc. in Telecommunication Engineering

Tribunal nombrado por el Mgfco. y Excmo. Sr Rector de la Universidad Politécnica de Madrid el día. de. de 2020 .

\section{Tribunal}

\author{
Presidente: NOMBRE APELLIDO \\ Vocal: NOMBRE APELLIDO \\ Vocal: NOMBRE APELLIDO \\ Vocal: NOMBRE APELLIDO \\ Secretario: NOMBRE APELLIDO \\ Suplente: NOMBRE APELLIDO \\ Suplente: NOMBRE APELLIDO
}

Realizado el acto de lectura y defensa de la tesis el día de

de 2020 .

Calificación de la Tesis......

El Presidente: Los Vocales: $\quad$ El Secretario: 

A mi amor, Andrius,

A mi querida hermanita, Marta, A mis queridos padres, Mari y Prudencio, $Y$ a todos mis amigos.

A todos ellos, que siguieron ahi, incluso cuando yo he ido $y$ venido durante estos años.

Chacho, ique ya acabé! 



\section{Resumen}

La expansión de los métodos de aprendizaje automático ha seguido un ritmo sin precedentes durante la última década. Su habilidad para resolver problemas de diversa naturaleza ha colocado a las técnicas de aprendizaje automático en el foco de numerosas líneas de investigación y proyectos industriales. Paralelamente, la investigación e innovación en sistemas robóticos ha estado constantemente creciendo, en términos de desarrollo de hardware y algoritmos clásicos. Sin embargo, el nivel de autonomía robótica proporcionado por algoritmos ajustados manualmente, con modelos del problema comúnmente simplificados, está alcanzando su límite tecnológico. En este contexto, los métodos de aprendizaje automático, tales como aprendizaje profundo y por refuerzo, han proporcionado resultados sobresalientes en escenarios complejos (p.ej. tareas de visión por computador), que requieren el tratamiento de información de alta dimensionalidad o fuentes heterogéneas de datos.

Siguiendo estas ideas, la presente tesis doctoral está enmarcada dentro de un novedoso paradigma que el campo de la robótica está actualmente explorando, donde los robots pueden aprender comportamientos de alto nivel en un entorno simulado, para posteriormente ser desplegados en un entorno real relevante. Por primera vez, se han usado métodos de aprendizaje profundo y por refuerzo para resolver aplicaciones complejas basadas en visión para robótica aérea, tales como aterrizaje de un multirrotor en una plataforma móvil o seguimiento de un multirrotor no cooperativo. Adicionalmente, han sido utilizadas técnicas de aprendizaje profundo y por refuerzo en el marco de detección de objetos en secuencias de vídeo. Todas las técnicas empleadas han sido diseñadas, implementadas y validadas exhaustivamente en una amplia variedad de escenarios reales y relevantes. Las mencionadas aplicaciones han sido formuladas como problemas basados en visión, con el objetivo de ser resueltas con un multirotor y/o sistema empotrado de bajo coste y comerciales.

En este trabajo doctoral, la aplicación de aterrizaje de un multirrotor sobre una plataforma móvil ha sido explorada haciendo uso de métodos de aprendizaje profundo por refuerzo. La técnica completa ha sido aprendida en un entorno simulado y desplegada en un entorno real. La estrategia ha sido validada de manera exhaustiva y comparada con numerosas técnicas clásicas para el aterrizaje de multirrotores, verificando aún más a fondo la efectividad de la misma. Además, la aplicación de seguimiento de un multirrotor no cooperativo ha sido solucionada mediante aprendizaje profundo y por refuerzo, lo cuál involucró un reto adicional debido a su naturaleza de alta dimensionalidad y al riesgo de colisión de la maniobra de seguimiento, entre otros. En una tendencia similar, se ha propuesto un método para la utilización de imágenes sintéticas fotorrealistas en el contexto de detección de objetos, con el requerimiento final de ser utilizado para imágenes reales.

Finalmente, en esta tesis, han sido investigadas diversas técnicas de aprendizaje profundo y por refuerzo para detección de objetos en vídeo, con el objetivo global de aprovechar la información temporal presente en los fotogramas de una secuencia de vídeo, para así re- 
ducir la latencia de procesamiento. La técnica ha sido inspirada por la falta de atención al contexto de un objeto que la visión humana exhibe mientras realiza un seguimiento focalizado. La implementación derivó en una reutilización dinámica de contexto a lo largo de los fotogramas, junto con una estructura temporal especial que disminuyó aún más el coste computacional durante el procesamiento del vídeo. A este respecto, se ha propuesto una técnica innovadora, donde una política generada por aprendizaje por refuerzo puede ser entrenada con una distribución de funciones de recompensa, siendo capaz de encapsular numerosos comportamientos en una única política. En tiempo de inferencia, la política puede ser condicionada a un único comportamiento dependiendo de los requerimientos de la aplicación. La técnica propuesta ha sido validada en el marco de la aplicación de detección de objetos en vídeo. Sin embargo, el método es suficientemente genérico para ser aplicado en otros escenarios relacionados con el aprendizaje por refuerzo.

La presente tesis por compendio está compuesta por tres publicaciones en revistas científicas peer-reviewed. Dichas publicaciones contribuyen de manera equitativa a satisfacer los objetivos de esta tesis doctoral, siguiendo una unidad temática clara y progresiva. Además, estas publicaciones han extendido el estado del arte en las mencionadas aplicaciones de robótica aérea y han contribuido al uso de información sintética en el marco de la robótica para el mundo real. 


\section{Abstract}

The expansion of machine learning methods has followed an unprecedented pace during the last decade. Their ability to solve problems in domains of diverse nature has placed machine learning techniques on the focus of several research lines and industrial projects. Besides, the research and innovation in robotic systems have been constantly growing, in terms of hardware and classical algorithmic development. Nevertheless, the level of robotic autonomy provided by hand-engineered algorithms, with commonly simplified models of the problem, is reaching a technological limit. In this context, machine learning methods, such as deep and reinforcement learning, have provided outstanding results in complex scenarios (e.g. computer vision tasks), which require the treatment of high-dimensional information or heterogeneous sources of data.

Following these ideas, the present doctoral thesis is framed in a novel paradigm which the robotics field is currently exploring, where robots can learn high-level behaviors in a simulated environment, in order to be finally deployed in a real-world relevant environment. For the first time, deep and reinforcement learning methods and simulated environments have been used to solve challenging vision-based applications in aerial robotics, such as multirotor landing on a moving platform and non-cooperative multirotor following. Additionally, deep and reinforcement learning techniques have been utilized in the scope of object detection in video sequences. All of the techniques have been designed, implemented, and thoroughly validated in a wide variety of real and relevant scenarios. The stated applications have been formulated as vision-based problems, in order to be solved with a low-cost and off-the-shelf multirotors and/or embedded systems.

In this doctoral work, the application of autonomous multirotor landing on top of a moving platform has been explored by making use of deep reinforcement learning methods. The complete approach has been learned in a simulated environment and deployed in a real environment. The strategy has been exhaustively validated and compared to several classical multirotor landing techniques, further verifying its effectiveness. In addition, the task of autonomous non-cooperative multirotor following has been solved through deep and reinforcement learning, which involved an increased challenge due to its higher dimensional nature and complexity of the maneuver, among others. In a similar trend, the complete application has been solely aided by synthetic information, such as low-level simulated states and images. Moreover, a method for the utilization of synthetic photorealistic images in the context of object detection has been proposed, with the final requirement of performing with real-world images.

Finally, in this thesis, deep and reinforcement learning techniques have been researched for video object detection, with the global aim of taking advantage of the temporal information present in the frames of a video sequence, in order to reduce the processing latency. The approach has been inspired by the lack of attention to the context of an object which the human vision can exhibit when performing a focused tracking. The implementation led to dynamic context reuse across frames, along with a special temporal structure that 
further reduced the computational cost during the video computation. On this subject, an innovative technique has been proposed, where a reinforcement learning policy can be trained with a distribution of reward functions, being able to encapsulate several behaviors in one unique policy. At inference time, the policy can be conditioned on one unique behavior depending on the requirements of the application. The proposed technique has been validated under the scope of the video object detection application. However, the method is generic enough to be applied in further reinforcement learning related applications.

This thesis by compendium is composed of three peer-reviewed scientific journal publications. Said publications equally contribute to satisfying the objectives of this doctoral thesis, following a clear and progressive thematic unity. Moreover, these publications have extended the state of the art in the stated aerial applications and have contributed to the usage of synthetic information in the scope of real-world robotics. 


\section{Preface}

The work for my doctoral research was carried out at the Center for Automation and Robotics (CAR) at the Universidad Politécnica de Madrid (UPM). First of all, I would like to thank the director of my doctoral thesis Prof. Pascual Campoy. I am profoundly grateful to you for giving me the opportunity to discover a field of science that I have loved during these years. Also, I would like to express my gratitude for providing both financial and strategic support, as well as the opportunity to take part in key projects and international collaborations which have pushed me to become a better student, researcher, and academic during the course of these years. I also would like to thank Prof. Martín Molina and Prof. Sergio Domínguez for their advice and help during the realization of this thesis.

Any great achievement in life, such as a doctoral thesis, entails outstanding people to support and contribute to its success. In this regard, I am proud to have become part of the Computer Vision and Aerial Robotics (CVAR) group which has gathered amazing researchers, colleagues, and friends. I would especially like to thank Hriday Bavle, for his unstoppable energy in every challenging and tough project or competition, part of my great successes are his too. I also would like to thank Carlos Sampedro, for his long-lasting and interesting talks about reinforcement learning, and Ramón Suárez Fernández, for his always-available support in any respect during the course of the thesis. A special thank to Javier Rodríguez Vázquez, for his proactivity, commitment, and energetic help, Adrián Álvarez, one of the best master students I have ever had the pleasure of supervising and an outstanding research/competition partner, and Lu Liang, always very supportive and interested in helping. I have to express my gratitude to Zorana Milosevic, Adrián Carrio, Miguel Fernández Cortizas, Ignacio Gil Moreno, Alejandro Garcia-Vaquero, Andrés Parra, Juan Cely, Ángel, Rosa Ortiz, Carlos Sánchez and all the people who were there to help and generate joyful moments during the long days in the lab.

Additionally, I would like to mention and especially thank the members of the Aerospace Controls Laboratory (ACL) of the Massachusetts Institute of Technology (MIT), where I conducted my research stay. I want to thank Prof. Jonathan P. How, for welcoming me to his outstanding research group, and Jesús Tordesillas Torres, for his active guidance and politeness during the process of my research stay. Also, I would like to thank Samir Wadhwania, Dong-Ki Kim, Michael Everett, Kris Frey, Parker Lusk, Aleix Paris, and Britton Bradley for their warm assistance during the stay.

Building a life and pursuing a doctoral degree out of the Canary Islands have been an incredible but, at the same time, tough journey. I would like to thank my friends (you know who you are) for the amazing moments we have shared. Of course, I want to thank my family for all of their support, encouragement, and love throughout my entire academic career. Mari and Prudencio, thank you for always being there for me, none of my accomplishments would ever have been possible without your constant sacrifices in several ways. Marta, my little sister, thank you for always being so supportive and caring. 
Andrea, my beloved girlfriend, thank you for always supporting and believing in me, even in the moments I made the things more difficult. Sorry for the missed moments and long days. You have inspired me during these eight years, and you keep on making me believe, everything is possible if shared with you. 



\section{Contents}

Resumen viii

\begin{tabular}{ll}
\hline Abstract & $\mathrm{x}$
\end{tabular}

Preface xii

$\begin{array}{ll}\text { List of Figures } & \text { xvi }\end{array}$

$\begin{array}{ll}\text { List of Tables } & \text { xxi }\end{array}$

List of Publications xxii

\begin{tabular}{ll}
\hline Acronyms & 1
\end{tabular}

\begin{tabular}{lll}
\hline & Introduction & 4
\end{tabular}

1.1 Motivation . . . . . . . . . . . . . . . . . . . 5

1.2 Historical Background $\ldots \ldots \ldots \ldots \ldots$. . . . . . . . . . . 6

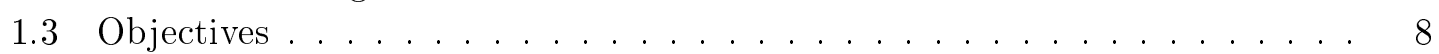

1.3 .1 Requirements . . . . . . . . . . . . . . . . . . . . . . . . . . . 9

1.4 Contributions $\ldots \ldots \ldots \ldots \ldots \ldots$

$1.5 \quad$ Outline $\ldots \ldots \ldots \ldots \ldots \ldots$

\begin{tabular}{llr}
\hline & Literature Review & 12
\end{tabular}

2.1 Classical Control Approaches for Autonomous UAV Landing and Object

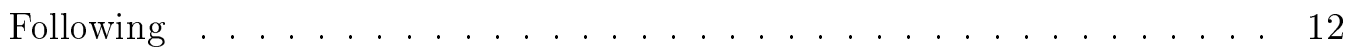

2.2 Machine Learning Approaches for UAV Control . . . . . . . . . . . . . . . . 14

2.3 Reinforcement Learning Frameworks for Robotics . . . . . . . . . . . . . . . 16

2.4 Synthetic Data for Real-World Robotics . . . . . . . . . . . . . . . . . . 17

2.5 Detection Methods for Flying UAVs $\ldots \ldots \ldots$. . . . . . . . . . . . . . . 19

2.6 Reinforcement Learning for Object Detection $\ldots \ldots \ldots$. . . . . . . . . . 20

\begin{tabular}{lll}
\hline 3 & Methodology & 22
\end{tabular}

3.1 Technology Readiness Levels . . . . . . . . . . . . . . . . . . . . . . . . . 25

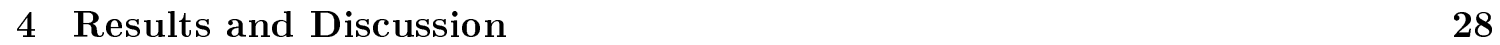

4.1 Overall Concepts . . . . . . . . . . . . . . . . . . . . . . . . . . . . . . . . . . . . 29

4.2 Reinforcement Learning Framework . . . . . . . . . . . . . . . . . . . . . . . 31

4.2 .1 Introduction . . . . . . . . . . . . . . . . . . . 31 
4.2 .2 Deep Deterministic Policy Gradients . . . . . . . . . . . . . . . . . 32

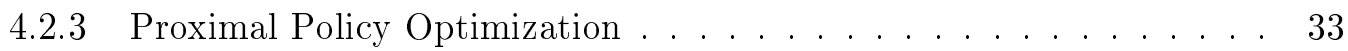

4.2 .4 Framework Design $\ldots \ldots \ldots \ldots$. . . . . . . . . . . . . . 33

$4.3 \quad$ Autonomous Landing on a Moving Platform (Publication P.I) . . . . . . . . 35

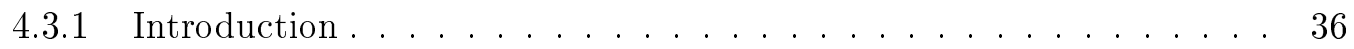

4.3 .2 Classical Methods . . . . . . . . . . . . . . . . . . 36

4.3 .3 Reinforcement Learning Based Methods . . . . . . . . . . . . . . . . 41

4.4 Autonomous Multirotor Following (Publication P.II) . . . . . . . . . . . . 47

4.4 .1 Introduction . . . . . . . . . . . . . . . . . . 47

4.4 .2 Deep Learning Based Methods . . . . . . . . . . . . . . . . . . . 48

4.4 .3 Reinforcement Learning Based Methods . . . . . . . . . . . . . . . . 50

4.5 Adaptive Video Object Detection (Publication P.III) . . . . . . . . . . . . . 52

4.5 .1 Introduction . . . . . . . . . . . . . . . . . 54

4.5 .2 Deep Learning Based Methods . . . . . . . . . . . . . . . . . 55

4.5 .3 Reinforcement Learning Based Methods . . . . . . . . . . . . . . . 56

$\begin{array}{lll}5 & \text { Conclusions and Future Work } & 63\end{array}$

$\begin{array}{lc}\text { Publications } & 66\end{array}$

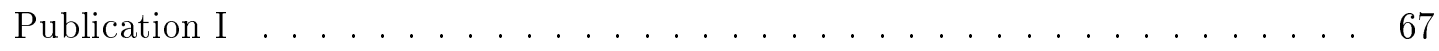

Publication II . . . . . . . . . . . . . . . . . . . . . . . . . . . . . . . 84

Publication III . . . . . . . . . . . . . . . . . . . 105

\begin{tabular}{lr}
\hline Appendices & 122
\end{tabular}

\begin{tabular}{|r|r|}
\hline A International Competitions & $\mathbf{1 2 3}$
\end{tabular}

A.1 2020 Mohamed Bin Zayed International Robotics Competition (MBZIRC) . 123

A.2 2018 IROS Autonomous Drone Race (IROS ADR) Competition . . . . . . . . 125

A.3 2017 International Micro Aerial Vehicles (IMAV) Competition . . . . . . . . . 126

A.4 2016 International Micro Aerial Vehicles (IMAV) Competition . . . . . . . . 127

\begin{tabular}{lr}
\hline B Industrial Project & $\mathbf{1 3 0}$
\end{tabular}

B.1 Autonomous UAV Power Plant Inspection . . . . . . . . . . . . . . . . . 130

\begin{tabular}{|r|r|}
\hline C Scientific Dissemination & 133 \\
\hline
\end{tabular}

C.1 Journal Publications . . . . . . . . . . . . . . . . . . . . . . . 133

C.2 Conference Publications . . . . . . . . . . . . . . . . . . . . . . 134

\begin{tabular}{lr}
\hline D Unpublished Results & $\mathbf{1 3 6}$
\end{tabular}

D.1 Domain Adaptation for Collision Detection . . . . . . . . . . . . . . . 136

\begin{tabular}{lr}
\hline Bibliography & 140
\end{tabular} 


\section{List of Figures}

1.1 Artificial intelligence partial taxonomy, with a special interest in the machine learning methods, primary techniques explored in this dissertation. . . . . . 5

1.2 Brief graphical history of milestones in machine learning research and development over the last century until nowadays. The year and authors of the contributions have been included for clarity. . . . . . . . . . . . . . 7

3.1 Methodology followed by the present thesis. It has resulted from a combination of SIMILAR methodology (Bahill and Gissing (1998)) and the spiral model of the software process $($ Boehm $(1988)) . \ldots \ldots \ldots 23$

3.2 Technology readiness levels as a reference of the state of the developed technology, during the course of the presented thesis study. . . . . . . . . . 26

4.1 Flow chart of the publications included in this thesis by compendium. The key differentiators and interconnections have been summarized. . . . . . . . 28

4.2 The base UAV system with four rotors (quadrotor) distributed in "X" shape. $T_{i}$ corresponds to the $i$-th thrust and $M_{i}$ to the $i$-th heading torque. $\ldots .29$

4.3 Basic cascaded control scheme for multirotor control. The publications P.I and P.II have been framed in the scope of velocity and attitude control,

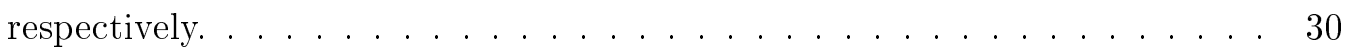

4.4 Reinforcement learning problem formulation. The basic structures are the agent and the environment. The agent provide actions $a_{t}$ to the environment which responds with the next state $s_{t}$ and the reward $r_{t} . \ldots \ldots 31$

4.5 Diagram of the proposed reinforcement learning framework. The theoretical concept of the reinforcement learning environment is highlighted in pale red. Simulation systems or multi-purpose architectures are highlighted in

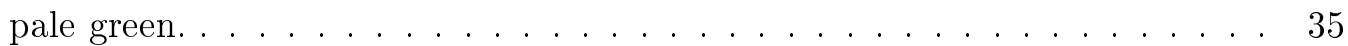

4.6 A real image composition of a multirotor landing on a moving platform using Rodriguez-Ramos et al. (2018) approach. . . . . . . . . . . . . . 36

4.7 All the components involved in the Rodriguez-Ramos et al. (2017) approach: estimation (Helipad Detector, Image-based Pose Extractor, Altimeter-based Velocity Extractor, Flight Altitude Estimator and Motion Estimator), prediction (Motion Predictor) and fast landing (Fast Landing). The preliminary components developed for the IMAV 2016 competition are highlighted

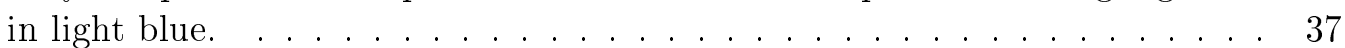


4.8 (a) Experiment conducted during the execution of a simulated flight with rectilinear and periodic trajectory of the MP. (Top) Ground truth and estimated velocity of the MP. The final value is $v_{M P}^{W}=0.66 \mathrm{~m} / \mathrm{s}$. (Bottom) Estimated absolute distance from the ground and 1D-LIDAR altimeter measurements. $t_{\text {land }}=58.37 \mathrm{~ms}$ is the instant of time when landing is triggered. (b) Experiment conducted during the execution of a real flight with rectilinear and periodic trajectory of the MP. (Top) Estimated velocity of the MP. The final value is $v_{M P}^{W}=0.3788 \mathrm{~m} / \mathrm{s}$. (Bottom) Estimated absolute distance from the ground and 1D-LIDAR altimeter measurements. $t_{\text {land }}=$ $153.68 \mathrm{~ms}$ is the instant of time when landing is triggered. . . . . . . . . . 38

4.9 Experiment conducted during the execution of a simulated flight with nonperiodic trajectory of the MP. (Top) Ground truth and estimated velocity of the MP. The converged value $\left(v_{M P}^{W}=0.278 \mathrm{~m} / \mathrm{s}\right)$ is taken to enable the fast landing stage. (Bottom) Estimated absolute distance from the ground and 1D-LIDAR measurements. $t_{\text {land }}=269.75 \mathrm{~ms}$ is the instant of time when

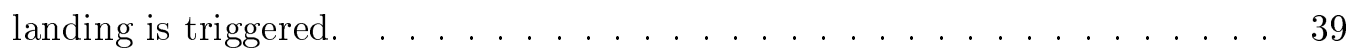

4.10 Simulation results corresponding to four different landing scenarios for PBVS approach. (a)(b) Rectilinear periodic trajectory of the MP with a maximum velocity of $0.4 \mathrm{~m} / \mathrm{s}$ (Slow). (c)(d) Rectilinear periodic trajectory of the MP with a maximum velocity of $1.2 \mathrm{~m} / \mathrm{s}$ (Fast). (e)(f) 8-Shape periodic trajectory (with constant angular velocity of $0.1 \mathrm{rad} / \mathrm{s}$ ) of the MP with a maximum velocity of $0.628 \mathrm{~m} / \mathrm{s}$ (8-Shape). (g)(h) Rectilinear non-periodic trajectory of the MP with a maximum velocity of $1.5 \mathrm{~m} / \mathrm{s}$ (Runaway). . . . 40

4.11 Real-flight results corresponding to two different landing scenarios. (a)(b) Rectilinear periodic trajectory of the MP with a maximum velocity of 0.8 $\mathrm{m} / \mathrm{s}$ (Fast). (c)(d) Rectilinear non-periodic trajectory of the MP with a maximum velocity of $0.86 \mathrm{~m} / \mathrm{s}$. Ground truth data was not available in this experiment. Instead, relative position with respect to the MP has been

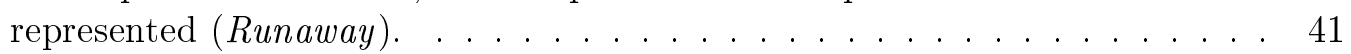

4.12 Architecture of the proposed P.I reinforcement learning architecture for the case of the experiment of study . . . . . . . . . . . . . . . 42

4.13 Average and standard deviation of the accumulated reward, for both simulated training and testing phases. . . . . . . . . . . . . . . . 42

4.14 Partial state and actions signals of policy network from episode 4500 in simulated environment. (Top) Position of the multirotor with respect to the $\mathrm{MP}$, in $\mathrm{x}$ and $\mathrm{y}$ axes. (Middle) Velocity of the multirotor with respect to the MP, in $\mathrm{x}$ and $\mathrm{y}$ axes. (Bottom) Velocity reference commands (actions) generated by the agent, in world coordinates and in $\mathrm{x}$ and $\mathrm{y}$ axes. . . . . . . 43

4.15 Partial state and actions signals of policy network from episode 4500 in a real-flight environment, for (a) a periodic trajectory of the MP and (b) an arbitrary trajectory of the MP. (Top) Position of the multirotor with respect to the MP, in $\mathrm{x}$ and $\mathrm{y}$ axes. (Middle) Velocity of the multirotor with respect to the MP, in $\mathrm{x}$ and $\mathrm{y}$ axes. (Bottom) Velocity reference commands (actions) generated by the agent, in world coordinates and in $\mathrm{x}$ and $\mathrm{y}$ axes. . . . . . . 44

4.16 Architecture of the proposed DRL based landing system in Rodriguez-Ramos et al. [2018). All the frames of reference involved and the networks structure

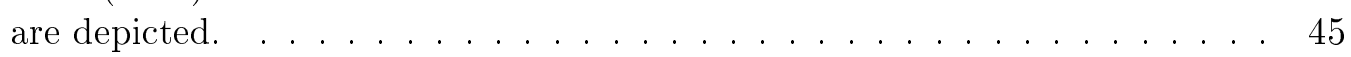


4.17 Simulation results corresponding to four different landing scenarios for DRL approach. (a)(b) Rectilinear periodic trajectory of the MP with a maximum velocity of $0.4 \mathrm{~m} / \mathrm{s}$ (Slow). (c)(d) Rectilinear periodic trajectory of the MP with a maximum velocity of $1.2 \mathrm{~m} / \mathrm{s}$ (Fast). (e)(f) 8-Shape periodic trajectory (with constant angular velocity of $0.1 \mathrm{rad} / \mathrm{s}$ ) of the MP with a maximum velocity of $0.628 \mathrm{~m} / \mathrm{s}(8-$ Shape $)$. (g)(h) Rectilinear non-periodic trajectory of the MP with a maximum velocity of $2 \mathrm{~m} / \mathrm{s}$ (Runaway). $\ldots . .45$

4.18 Real-flight results corresponding to two different landing scenarios. (a)(b) Rectilinear periodic trajectory of the MP with a maximum velocity of 0.8 $\mathrm{m} / \mathrm{s}$ (Fast). (c)(d) Rectilinear non-periodic trajectory of the MP with a maximum velocity of $0.86 \mathrm{~m} / \mathrm{s}$. Ground truth data was not available in this experiment. Instead, relative position with respect to the MP has been

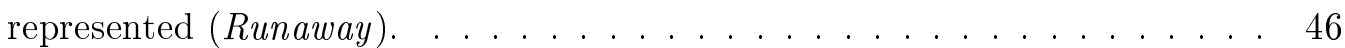

4.19 Image composition of a non-cooperative multirotor (light-blue) followed by another multirotor that implemented the P.II synthetic-learning based approach (light-green) in the real experimentation environment. . . . . . . . . 47

4.20 Architecture of proposed multirotor detector, based on RetinaNet architecture, with ResNet50 backbone and five levels for Feature Pyramid Network (FPN; fewer levels represented in figure for the sake of simplicity). Backbone weights were frozen during training. Final subnetworks were fine-tuned with pure synthetic multirotor images. . . . . . . . . . . . . . 48

4.21 Examples of the synthetic images corresponding to the generated dataset. . 49

4.22 Examples of the real images corresponding to the validation dataset. Both \begin{tabular}{|l}
\hline true positives and false negatives have been shown for the multirotor, bird \\
\hline \hline and car classes. . . . . . . . . . . . . . . . . 50
\end{tabular}

4.23 Architecture of the proposed DRL-based autonomous multirotor following task, included in P.II. All the frames of reference involved and the networks structure are depicted. . . . . . . . . . . . . . . . 51

4.24 Simulation results corresponding to four different scenarios for the P.II multirotor following approach. (a)(b)(e)(f) Rectilinear periodic trajectory of the Non-Cooperative Multirotor (NC-M) along the $\mathrm{x}$ and $\mathrm{y}$ axes with a maximum velocity of $1.3 \mathrm{~m} / \mathrm{s}(X$-Axis and $Y$-Axis, respectively). (i)(j) Rectilinear periodic trajectory of the NC-M along $\mathrm{z}$ axis with a maximum velocity of 0.5 $\mathrm{m} / \mathrm{s}($ Z-Axis $) .(\mathrm{m})(\mathrm{n})$ Arbitrary non-periodic trajectory of the NC-M with a maximum velocity of $0.47 \mathrm{~m} / \mathrm{s}($ Arbitrary). $(\mathrm{c})(\mathrm{d})(\mathrm{g})(\mathrm{h})(\mathrm{k})(\mathrm{l})(\mathrm{o})(\mathrm{p})$ Currenttarget RoI center and area error corresponding to each of the four stated experiments. Multirotor trajectories have been generated by a groundtruth

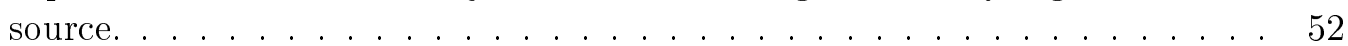

4.25 Real-flight results corresponding to four different scenarios for the P.II multirotor following approach. (a)(b)(e)(f)(i)(j) Rectilinear periodic trajectory of Noncooperative Multirotor (NC-M) along $\mathrm{x}, \mathrm{y}$, and $\mathrm{z}$ axes with a maximum velocity of $0.3 \mathrm{~m} / \mathrm{s}(X$-Axis, $Y$-Axis and Z-Axis, respectively). $(\mathrm{m})(\mathrm{n})$ Arbitrary nonperiodic trajectory of NC-M with maximum velocity of 0.3 $\mathrm{m} / \mathrm{s}($ Arbitrary). (c) (d)(g)(h)(k)(l)(o)(p) Current-target RoI center and area error corresponding to each of four stated experiments. Multirotor trajectories have been generated by an Extended Kalman Filter (EKF) that utilizes optical-flow velocities and measured accelerations as only input source. . . . 53 
4.26 A schematic diagram of the P.III proposed approach. The keyframe con\begin{tabular}{|l|}
\hline text is dynamically reused to reduce computation overhead, and temporal \\
\hline \hline information is propagated by the temporal aggregator. Grey region in an \\
\hline \hline image frame denotes pixels which are not being processed for the given frame. 54 \\
\hline 4.27 A block diagram of our adaptive keyframe selection technique with all the \\
\hline components of the framework included, where $f$ stands for feature extrac- \\
\hline \hline tor, $c$ is the context aggregator, $a$ depicts the temporal aggregator, and $d$ \\
\hline \hline represents the detector. For clarity, coloured blocks correspond to tensors \\
\hline \hline and grey blocks correspond to components of the system. The length of \\
\hline \hline the tensor denotes time consumption but it is not to scale. The context \\
\hline of a keyframe $F_{k}^{c}$ is not being modified throughout the pipeline of context \\
\hline aggregators. $\ldots \ldots \ldots \ldots \ldots \ldots \ldots \ldots \ldots \ldots \ldots \ldots \ldots \ldots \ldots \ldots \ldots \ldots \ldots$ \\
\hline
\end{tabular}

4.28 Representation of the trade-off between the mAP and the percentage of inattentional frames executed. Four cases with two feature extractors (MobileNetV1 and MobileNetV2) and two datasets (Imagenet VID 2015 and MAV-VID) have been evaluated. The random baseline policy is depicted in pale blue (circles) and the proposed inattentional policy in pale green (triangles). The original mAP (with no inattentional frames involved) is represented with a grey dashed line. The inattentional policy predominates allover the tests, resulting in a higher mAP for every percentage of inattentional frames. Also, the inattentional policy incurs less accuracy degradation even at extreme ratios of inattentional frames, suggesting that P.III method is superior at capturing the temporal dynamics inherent to videos. Every test has been performed 5 times each, for both policies, and the average results have been plotted. . . . . . . . . . . . . . . . 60

4.29 Images corresponding to four different scenarios for both datasets. Every image example has been processed by MobileNetV1-ConvLSTM-SSDLite $\left[\lambda_{0}=1.2\right]$ model (MobileNetV2-ConvLSTM-SSDLite provides a similar behavior). Shaded regions correspond to non-processed pixels. (a)-(b) and (c)-(d) image examples correspond to Imagenet VID 2015 and MAV-VID dataset, respectively. (a) and (c) show complex detection examples, corresponding to an object with high-motion speed within the image plane, and to an increased environmental complexity (camera pointing to the sun), respectively. In this context, the inattentional policy executes a lower percentage of inattentional frames. (b) and (d) illustrate objects which remain more static throughout the sequence. In this scenario, the number of inattentional frames is higher. . . . . . . . . . . . . . 61

A.1 (a) Designed UAV platform with hardware components highlighted. (b) Gripper CAD model. . . . . . . . . . . . . . . . . . . 124

A.2 Results from an autonomous grasping maneuver test in an outdoor experimentation environment. (a)(c)(e) Sequence view of the aerial vehicle grasping maneuver. (b)(d)(f) Images from the on-board camera used for detection of the target UAV and ball. . . . . . . . . . . . . . . . . . . . 124

A.3 (a) (b) UAV in the real competition environment. . . . . . . . . . . . . . . 125

A.4 Utilized UAV platform (Parrot Bebop 2) with hardware components (Intel

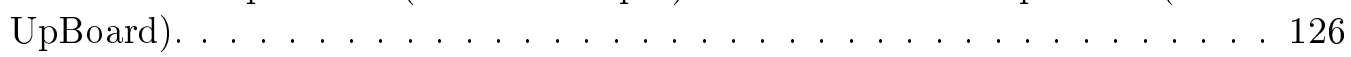

A.5 Simulated and real replica of the IROS ADR 2018 environment (a) Simulated environment. (b) Real environment. . . . . . . . . . . . . 126 
A.6 Utilized UAV platform (Parrot Bebop 2) with hardware components (Parrot Slam Dunk). . . . . . . . . . . . . . . . . . 127

A.7 Real competition environment for the IMAV 2017 indoor competition. . . . 127

A.8 Designed UAV platform with hardware components highlighted. . . . . . . . . 128

A.9 Simulated replica of the IMAV 2016 indoor competition environment. . . . . 128

A.10 Real partial replica of the IMAV 2016 indoor environment (a) The UAV is autonomously navigating through an unstructured and cluttered indoor environment. (b) The UAV is autonomously landing on a moving platform, utilizing technique provided in Section 4.3 .2 . . . . . . . . . . . . 129

B.1 Representative image of the developed UAV platform (DJI Matrice 100) in the outdoor surroundings of the power plant. . . . . . . . . . . 131

B.2 Implemented UAV platform with hardware components. . . . . . . . . . . . 131

B.3 Simulated and real-flight experiments of the autonomous UAV navigation in a power plant boiler. (a) Simulated trajectory of the UAV (ground-truth and estimated) and 1D-LIDAR altimeter measurements. (b) Real-flight trajectory of the UAV (estimated) and 1D-LIDAR altimeter measurements. . . 132

D.1 Unpaired training images from two domains. $X$ corresponds to the realdomain images extracted from a public dataset. $Y$ corresponds to the synthetic-domain images generated under Unreal game engine. . . . . . . . . 137

D.2 Domain adaptation model architecture. The model contains two mapping functions $G: X \rightarrow Y$ and $F: Y \rightarrow X$, and associated discriminators $D_{Y}$ and $D_{X} . D_{Y}$ encourages $G$ to translate $X$ into outputs indistinguishable from domain $Y$, and vice versa for $D_{X}$ and $F$. To further regularize the mappings, two cycle consistency losses were introduced, which capture the intuition that whether one domain is translated to the other and back again it should arrive at where it started: (b) forward cycle-consistency loss: $x \rightarrow$ $G(x) \rightarrow F(G(x)) \approx x$, and (c) backward cycle-consistency loss: $y \rightarrow F(y) \rightarrow$

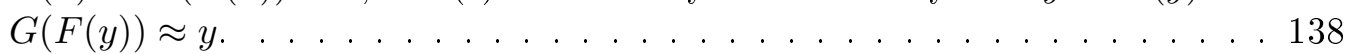

D.3 Examples of domain-adapted images. The synthetic images have been translated into the real domain in order to train a model for collision detection. . 138 


\section{List of Tables}

3.1 Technology readiness levels table of definitions. . . . . . . . . . . . . . . 27

4.1 Average Precision (AP) was computed for a minimum Intersection over Union (IoU) of [0.5:0.05:0.95], 0.5 and 0.75 (AP, $\mathrm{AP}_{50}$ and $\mathrm{AP}_{75}$, respectively). The network of epoch 0 corresponds to a special case, where network was not yet trained with synthetic images (only with real images of non-multirotor classes from COCO dataset) and was listed to make more salient the effect of our synthetic dataset. The network of epoch 4 was selected for experimentation due to its capability of generalization. . . . . . 49

4.2 Results using the MobileNetV1 feature extractor on the ImageNet VID 2015

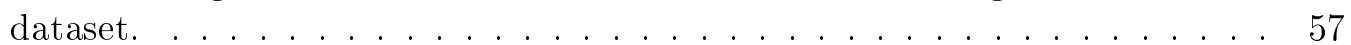

4.3 Results using the MobileNetV1 feature extractor on the MAV-VID dataset. 58

4.4 Results using the MobileNetV2 feature extractor on the ImageNet VID 2015

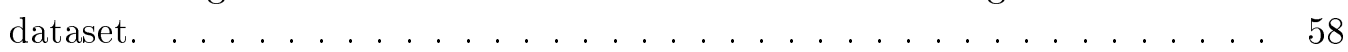

4.5 Results using the MobileNetV2 feature extractor on the MAV-VID dataset. 59

D.1 Accuracy results on the test dataset. The real dataset corresponds to a public dataset. The synthetic and domain-adapted dataset were generated based on Unreal game engine. . . . . . . . . . . . . . . . . . . . . . . . 139 


\section{List of Publications}

This thesis by compendium is based on the work and results presented in the following three journal publications, which best summarize and highlight the entire scientific dissemination shown in Appendix C, hereby referenced in the text as P.I - P.III:

P.I Rodriguez-Ramos, A., Sampedro, C., Bavle, H., De La Puente, P., and Campoy, P. (2018). A deep reinforcement learning strategy for UAV autonomous landing on a moving platform. Journal of Intelligent \& Robotic Systems, 93(1-2), 351366. doi:10.1007/s10846-018-0891-8. JCR (2019): 2.890, Manufacturing Engineering (Q1), Artificial Intelligence (Q2).

P.II Rodriguez-Ramos, A., Alvarez-Fernandez, A., Bavle, H., Campoy, P., and How, J. P. (2019). Vision-based multirotor following using synthetic learning techniques. Sensors, 19(21), 4794. doi: 10.3390/s19214794. JCR (2019): 3.275, Instruments and Instrumentation (Q1), Engineering, Electrical \& Electronic (Q2).

P.III Rodriguez-Ramos, A., Rodriguez-Vazquez, J., Sampedro, C., and Campoy, P. (2020). Adaptive Inattentional Framework for Video Object Detection With Reward-Conditional Training. IEEE Access, 8, 124451-124466. doi: 10.1109/ACCESS.2020.3006191. JCR (2019): 4.670, Computer Science (miscellaneous) (Q1), Engineering (miscellaneous) (Q1).

Publication P.I proposed an original deep reinforcement learning based approach for autonomous multirotor landing on top of a moving platform. The publication laid the foundations of a novel reinforcement learning framework, which facilitates the research and prototyping of deep reinforcement learning algorithms for real-world aerial robotics applications. Also, the publication introduced the basic formulation for the successful training of an aerial robot in a simulated environment in order to be deployed in real environments. A wide variety of experiments were performed to validate the approach in both simulated and real environments.

Publication P.II continued the research and development of the deep reinforcement learning based approach in the context of the application of non-cooperative multirotor following. The application involved an increased complexity, due to the high amount of degrees of freedom and collision risk of the maneuver. Additionally, a novel technique for the usage of synthetic photorealistic images in a conventional deep learning based object detector training has been proposed. The complete application was approached from a vision-based perspective. A wide variety of experiments in both simulated and real-flight scenarios have been successfully carried out in order to validate the technique.

Publication P.III continued the research and development of the deep learning based object detection approach by exploiting the temporal information normally available in video 
sequences. The approach was inspired by the human vision, which presents some lack of attention to the context of an object when performing a focused tracking. The implementation led to context reuse across frames, incurring a latency reduction of the video processing. In addition, a novel technique within the reinforcement learning paradigm has been proposed, where an agent can be trained not only with a unique reward function but with a complete distribution of reward functions, allowing for the encapsulation of several behaviors in one unique agent. The complete approach has been tested and validated in a wide variety of conditions, datasets, and system architectures.

In all publications P.I - P.III, the author of this thesis was responsible for the design, implementation, testing, and validation of the approaches, as well as of the design and writing of the manuscripts. The co-authors in every publication have greatly contributed to the discussion and implementation in every stage of the development, as well as in the assistance in the development and revision of the manuscripts, further increasing the quality of the techniques and their dissemination. 


\section{Acronyms}

ACER Actor-Critic with Experience Replay

AGI Artificial General Intelligence

AI Artificial Intelligence

AP Average Precision

API Application Programming Interface

AUV Autonomous Underwater Vehicles

CAD Computer-Aided Design

cGAN cycle Generative Adversarial Network

CMT Consensus-based Matching Tracker

CNN Convolutional Neural Network

COCO Common Objects in COntext

CPU Central Processing Unit

DDPG Deep Deterministic Policy Gradients

DL Deep Learning

DNN Deep Neural Network

DOF Degrees Of Freedom

DPM Deformable Part Model

DQN Deep Q-Network

DQRN Deep Recurrent Q-Network

DRL Deep Reinforcement Learning

FCN Fully Convolutional Network

FCRN Fully Convolutional Regression Network

FPS Frames Per Second

GAN Generative Adversarial Network 
GPS Global Positioning System

GPT Generative Pre-trained Transformer

GPU Graphical Processing Unit

HOG Histogram Of Gradients

IBVS Image-Based Visual Servoing

IMAV International Micro Aerial Vehicles

IMU Inertial Measurement Unit

IoU Intersection over Union

LBP Local Binary Patterns

LGVC Laser Gazed Viewing Camera

LIDAR Laser Imaging Detection and Ranging

LQR Linear-Quadratic Regulator

LSPI Least Square Policy Iteration

LSTM Long Short-Term Memory

mAP mean Average Precision

MAV Micro Aerial Vehicle

MBZIRC Mohammed Bin Zayed International Robotics Challenge

ML Machine Learning

MP Moving Platform

MPC Model Predictive Controller

NC-M Non-Cooperative Multirotor

PBVS Position Based Visual Servoing

PID Proportional-Integral-Derivative

PPO Proximal Policy Optimization

RGB Red Green Blue

RoI Region of Interest

ROS Robotic Operating System

RPT Robust and Perfect Tracking

SLAM Simultaneous Localization And Mapping

SotA State of the Art 
SITL Software In The Loop

SNN Spinking Neural Network

TLD Tracking-Learning-Detection

TRL Technology Readiness Level

TRPO Trust Region Policy Optimization

UAV Unmanned Aerial Vehicle

UGV Unmanned Ground Vehicle

YOLO You Only Look Once

YOTO You Only Train Once 


\title{
Chapter 1
}

\section{Introduction}

\author{
All progress is born of inquiry. Doubt is often \\ better than overconfidence, for it leads to \\ inquiry, and inquiry leads to invention
}

Hudson Maxim

The present Doctoral Thesis aims at contributing to several aspects of autonomous aerial-robots navigation by working in the cutting-edge technologies of Deep Learning (DL) and Deep Reinforcement Learning (DRL), part of the growing Artificial Intelligence (AI) field. This thesis is framed in a novel paradigm which the robotics field is currently exploring, where robots can learn high-level behaviors in a simulated environment, in order to be finally deployed in a real-world relevant environment. For the first time, DL and DRL methods and simulated environments have been used to solve challenging visionbased applications in aerial robotics, such as multirotor landing on a moving platform, non-cooperative multirotor following, and adaptive video object detection.

Originally, AI term, which is broadly used nowadays in several research and industry related topics, was coined in the 1956 Dartmouth Summer Research Project McCarthy et al. (2006) as a formal field. Currently, the term is considered over-utilized and covers a wide spectrum of techniques and algorithms, from symbolic methods, such as linguistics, formal logic, automated reasoning, or ontologies, to statistical methods, such as machine learning, pattern recognition, data mining, or probabilistic models. In the scope of the present dissertation, AI term is particularly related to Machine Learning (ML) techniques, and more concretely to DL and DRL methods and algorithms (see Fig. 1.1).

Particularly, in this dissertation, the DL methods are referred to supervised learning techniques in which multiple levels of simple but non-linear modules are composed (i.e. deep neural networks) to generate an abstraction of a representation LeCun et al. (2015). The DRL methods correspond to state-of-the-art reinforcement learning techniques in which the representation of non-linear functions is carried out by a deep neural network. For both DL and DRL methods, the non-linear modules present in a deep neural network vary from a wide spectrum, for instance, from fully connected or convolutional LeCun et al. (1998) to residual modules He et al. (2016). 


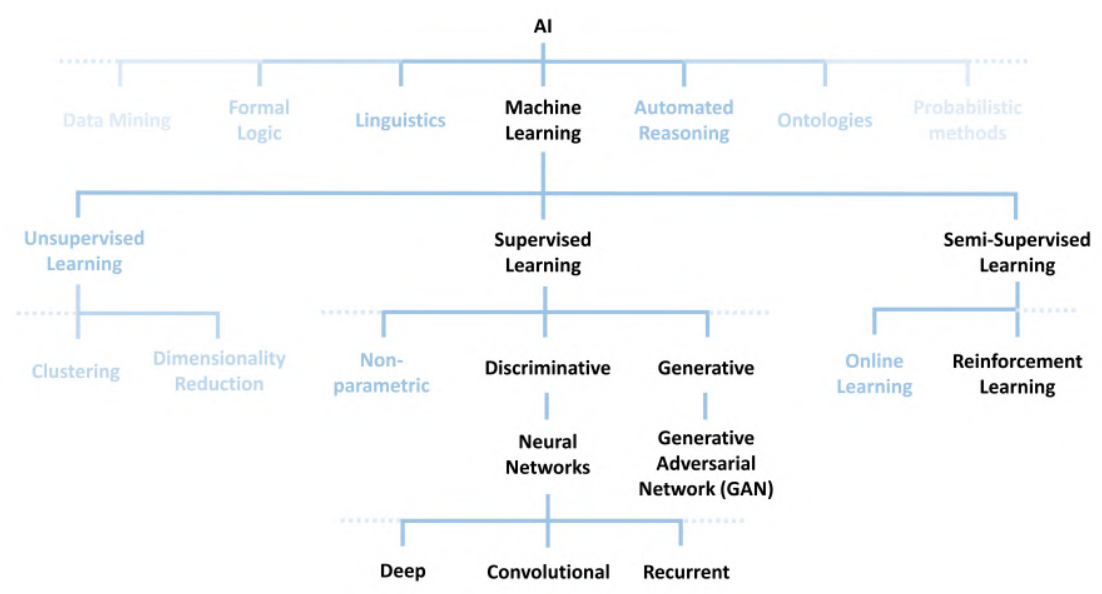

Figure 1.1: Artificial intelligence partial taxonomy, with a special interest in the machine learning methods, primary techniques explored in this dissertation.

\subsection{Motivation}

The expansion of AI, and in particular of ML methods, has followed an unprecedented pace which is believed to continue increasing in the upcoming years Mou (2019). Their ability to solve problems of diverse nature has placed ML techniques on the focus of different research topics in the community. Indeed, their advancements have evolved from purely theoretical aspects to more real-world solving algorithms. In the context of robotics, ML has been playing a growing role over the last decade.

The robotics field has also separately evolved but, as usual in hardware-related research and development, the evolution of the field takes more time to consolidate. On this basis, the robotics field has been aided by various techniques, from advanced control and planning to novel state estimation and Simultaneous Localization and Mapping (SLAM) approaches. All stated methods have shown outstanding results when solving real-world particular problems, and have provided an immense amount of knowledge during their course. However, robotics may seem to be approaching a limit where heuristics, handengineered methods, or simplified models of reality are not enough for a certain degree of autonomy. In this trend, ML techniques have revolutionized some fields, such as, for instance, computer vision, where traditional methods failed to fully absorb the high variability found in real-world imaging. ML approaches are able to learn from experience, in order to build a custom highly non-linear model of the problem under research, potentially able to solve it more accurately. In addition, on the way to Artificial General Intelligence (AGI), a subgroup of the research community believes that AI techniques must be deployed on robots in order to achieve a higher level of intelligence, since computer-simulated problems for AI are simpler and, thus, the AI can find shortcuts to the solution, incurring in a lower level of consciousnes: 1 . These facts, among others, constitute the primary aspects that motivate the present thesis study.

Furthermore, theoretical or computer-simulated ML research, albeit tackling other complex problems, skips the challenges of implementation, integration, and deployment of algorithms for real-world robotics, which normally incurs additional problems due to the required robustness against the wide variability of the environment and scarce computational resources. Concretely, the present dissertation not only aims at the research and

1 https://youtu.be/eKaYnXQUb2g 
development of practical ML methods for robotics but also the validation of stated methods in real and relevant experimentation environments. In addition, the complete dissertation is framed in the scope of aerial robotics (i.e. multirotor aerial robots). These aerial robots, also known as Unmanned Aerial Vehicles (UAVs), constitute a matter of special interest due to their high maneuverability and versatility in the context of complex tasks. The 6-Degrees Of Freedom (DOF) design of a multirotor allows for both precise and slow maneuvers, and fast and aggressive navigation or environment interaction, which can be considerably useful in the context of field robotics. Conversely, multirotor systems are unstable without adequate control and provide fast non-linear dynamics which are challenging to control in certain scenarios. Besides, most of the ML-related research is aided by powerful Graphical Processing Units (GPUs) for both training and inference purposes. Although embedded GPU systems are being actively improved, the development and implementation of DL or DRL algorithms to be deployed on an embedded system, mounted on an aerial robot, compose itself an additional challenge, due to computational and payload constraints. On this line, the present dissertation has worked towards the ultimate deployment of the developed algorithms on embedded systems, in order to be formally validated in real-world environments.

Finally, one common aspect of DL or DRL methods is the necessity of a high amount of data to be able to properly generalize for a given task. The stated amount of structured or annotated data may be not available at the desired scale or can be directly nonexistent. Also, in the context of DRL methods for robotics, where an agent is meant to safely interact with an environment an elevated number of times, the problem can become intractable in certain real-world scenarios during the training phase, due to safety constraints, system recovery, or energy resources. Nevertheless, synthetic data for real-world robotics is a recent field that is gaining traction due to its potential benefits in the context of massive data generation. Synthetic information can be notably useful for robots, which are potentially able to learn in a simulated environment where, for instance, time can be speed-up and several parallel instances of the robot can be run to increase learning pace. In this trend, simulators are constantly improving in order to provide higher levels of realism both in terms of physics simulations and photorealistic imaging. Following these ideas, the present study has explored several strategies to incorporate synthetic information into the learning process of aerial robots, in order to be deployed in the real world.

\subsection{Historical Background}

In this section, a brief explanation of important historical milestones in ML research and development is provided, yielding to the most recent ML achievements and their contributions to the robotic field and the specific objectives of this thesis. The most relevant milestones, in terms of the affinity of scope with the present dissertation, have been included. ML methods have experimented a rapid evolution over the last 80 years (see Fig. 1.2 , starting with the definition of the first mathematical model of a neuron in the Electronic Brain of McCulloch and Pitts (1943). In 1970, automatic differentiation methods are proposed Linnainmaa (1970). Nine years later, the Neocognitron Fukushima (1988) is proposed, being one of the precursors of the current Convolutional Neural Network (CNN) architectures. In 1986, backpropagation is popularized through distributed automatic differentiation for a Multi-Layer Perceptron Hinton et al. (1986), and three years later, a CNN is successfully used for handwritten character classification LeCun et al. (1989). With the advancement in computational resources, deep learning is born Salakhutdinov and Hinton (2009) and the first notable results are provided by AlexNet Krizhevsky et al. (2012) for 
image classification. Since then, novel architectures are constantly being researched for different purposes, such as the Generative Adversarial Networks (GANs) Goodfellow et al. (2014). Deep reinforcement learning took a major step in 2015 with Deep Q-Network Mnih et al. (2015), achieving human-level performance in arcade computer games, and in 2016, where the first computer program based on deep and reinforcement learning won a professional Go player in such a complex board game. In this trend, the computational resources have kept continuously growing, as well as the number of parameters of the models trained. At present, in 2020, activism for ethical AI has raised, and billion-parameters models, such as GPT-3 Brown et al. (2020) are providing unprecedented results in the field of Natural Language Processing (NLP).

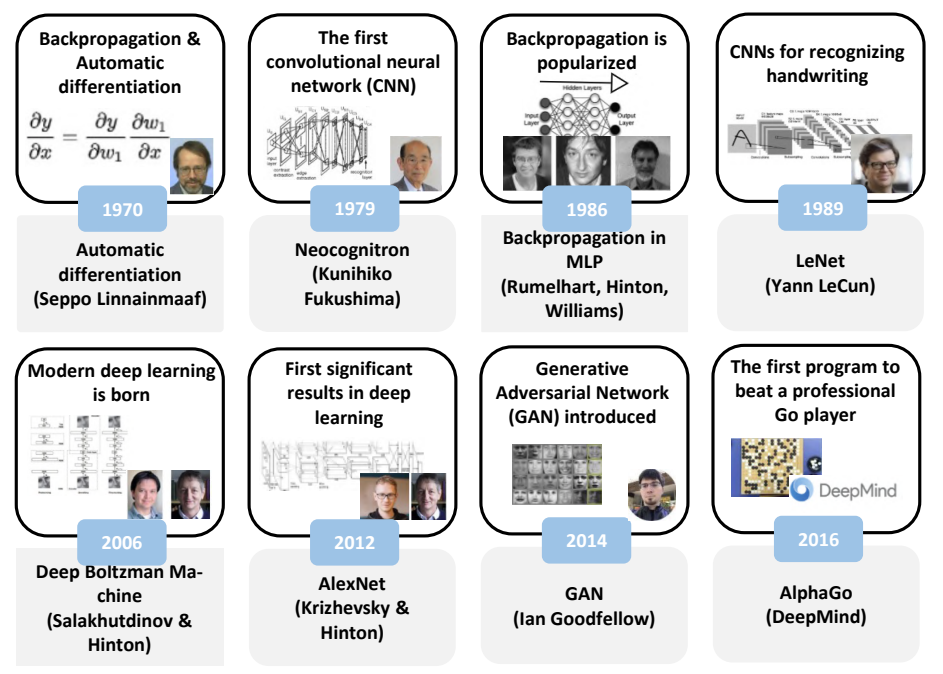

Figure 1.2: Brief graphical history of milestones in machine learning research and development over the last century until nowadays. The year and authors of the contributions have been included for clarity.

It has to be noted that the stated evolution of ML techniques could have not been possible without the parallel growth of hardware resources. In particular, the outstanding performance increase in Central Processing Units (CPUs), as well as in GPUs has been crucial for such an elevated growth of ML methods. Besides, mostly in the industry, the robotics field has also advanced in terms of hardware integration or tasks that a robot is able to solve (with classical control, planning, state estimation, and perception techniques). Among several other advances in the automation of industrial manufacturing facilities, articulated robotic arms Bischoff (2009), for instance, have encouraged the integration of complementary robots in the industry, where tasks ought to be precise, or can be dangerous for human employees. Furthermore, aforementioned hardware and materials improvements have led to the successful development of agile quadrupedal robots Hutter et al. (2016) or athletic humanoids Feng et al. (2014). In addition, in the last decade, there has been an exponential growth on the research of Unmanned Ground Vehicles (UGVs) Hebert et al. (2012); Shimoda et al. (2005); Matthies et al. (1996), Autonomous Underwater Vehicles (AUVs) Wynn et al. (2014); Marani et al. (2009); Fernandez et al. (2018) and UAVs $\mathrm{Hu}$ et al. (2015); Valavanis and Vachtsevanos (2015); Anderson and Gaston (2013), due to the increasing interest in their outstanding versatility, scalability and automation possibilities.

Nevertheless, despite great advancements in both ML and robotics fields, the highly non-linear function approximation capabilities of ML methods, as well as the possibility of directly learning from a given environment or robot model, have not fully been exploited in 
the robotics field. This fact, among several others, leads to a potential innovation domain, in-between these two research fields, which the present dissertation has aimed at as its primary contribution.

\subsection{Objectives}

The main objective of this thesis by compendium, addressed in the included publications (P.I - P.III), can be summarized in a single sentence: to explore and validate DL and DRL related methods in the context of aerial robotics, to solve novel and challenging applications, such as multirotor landing on a moving platform, non-cooperative multirotor following and video object detection in embedded systems; and with the ultimate goal of performing the experimentation in a real and relevant environment. In order to accomplish such a large scope objective, this dissertation seeks to attain the following sub-objectives:

O.1 Generation of Fully-Autonomous Behaviors The generation of fully autonomous behaviors for aerial robotics is a priority. This fact involves no human intervention during experimentation, both at an application or software component level in the context of complex missions. Furthermore, the developed techniques are aimed at having the final objective of targeting the maximum level of autonomy in the aerial robotics field.

O.2 Integration of Synthetic Data and Simulations Synthetic data, in the scope of both physics and photorealistic simulations, are aimed at improving the results of training procedures. Novel techniques shall be developed in order to properly take advantage of the synthetic information, with the ultimate objective of performing with real-world data.

O.3 Innovation on Detection Methods The present work shall explore ML related methods for vision-based applications. More concretely, ML-based techniques shall be exploited and improved in order to contribute to the object detection field, increasing the efficiency of the methods and providing solutions when the real-domain data is not available, among others.

O.4 Innovation on Control Methods The present work shall also explore ML related methods for control in the field of aerial robotics. More concretely, ML-based techniques shall be exploited and improved in order to provide control solutions to novel applications, providing improved behaviors and integrating sources and actions of heterogeneous natures.

O.5 Real-Environment Validation Every contribution of the present thesis shall end with real-world experimentation data, in order to satisfy one of the key motivation of the present dissertation, which is the usage of ML related methods to improve realworld applications. Furthermore, the approaches shall be designed and validated in embedded systems that shall be compatible with robotics development.

O.6 Community Contribution The proposed methods and techniques are aimed at complying with repeatability, replicability, and reproducibility constraints. Furthermore, to the extent possible, the developed approaches and gathered data shall be made publicly available to the community. Open-source and open data are primary priorities of this work. 


\subsubsection{Requirements}

The aforementioned objectives need to be achieved within the scope of a number of constraints and requirements. These are as follows:

R.1 The proposed technique(s) should be developed within the scope of aerial robotics.

R.2 The method(s) and algorithm(s) developed should be validated in fully autonomous mode.

R.3 The formal validation of the method(s) developed should be carried out in an embedded system or aerial robot.

R.4 The formal validation of the algorithm(s) should be performed in a general environment and under stress conditions that are able to extract the benefits and drawbacks of the $\operatorname{approach}(\mathrm{es})$.

R.5 Simulations are encouraged for the development of the method(s) and technique(s). However, final validation in a real environment (or embedded system) is compulsory.

R.6 The application(s) under research should be selected based on notable industrial or scientific interest.

R.7 Every contribution should be innovative in one or more fields and should be fully repeatable, replicable, and reproducible.

\subsection{Contributions}

The main contributions of this thesis by compendium focus on the design, development, test, and validation of ML-based techniques for aerial-robot control and object detection. These are as follows:

C.1 A solution for the task of landing on top of a moving platform, by means of DRL techniques for aerial motion control, has been proposed. The complete behavior has been trained in a simulated environment (with synthetic low-level information) and successfully tested in real-world relevant environments. The full problem has been tackled, with continuous state and action spaces and in fully-autonomous mode. This contribution is addressed in P.I.

C.2 A solution for the task of vision-based object following of a non-cooperative multirotor, by means of DRL techniques for aerial motion control, has been proposed. The complete behavior has been trained in a simulated environment (with synthetic low-level and RGB images information). The approach has been formulated as an image-based task and has incorporated a camera gimbal (2-DOF) to the problem. It has been successfully tested in real-world relevant environments. The full problem has been tackled, with continuous state and action spaces and in fully-autonomous mode. This contribution is addressed in P.II.

C.3 The usage of synthetic information is a primary contribution of this dissertation. A workflow for the usage of data from robotics simulations (physics level) in order to perform the training phase (to be finally deployed in real relevant environments) has been addressed in P.I and P.II. Additionally, synthetic images have been also 
integrated into the learning workflow. One method to that aim, where synthetic images from a game-engine simulation aid the object detection technique for real objects, has been proposed in P.II. Finally, intermediate synthetic feature maps were generated in P.III to increase the efficiency in the context of video object detection.

C.4 This dissertation, together with Sampedro et al. (2019), has greatly contributed to the original design, development, implementation, and maintenance of a DRL framework. The stated framework is described in P.I and P.II and aims at the ease of development of DRL based methods for aerial robotics. It includes several state-ofthe-art DRL algorithms and it is integrated with a renowned robotics simulator (and in particular with aerial-robot environments). Indeed, the stated DRL framework has contributed to the development of several publications Rodriguez-Ramos et al. (2018); Sampedro et al. (2018b a), a Ph.D. thesis Pérez (2019) and other M.Sc. thesis Zafra (2020).

C.5 A novel method for efficient video object detection has been proposed in P.III. With the reuse of context from certain frames in a video sequence, this approach is able to reduce the computation overhead. Furthermore, DRL techniques were integrated to adaptively select keyframes for context reuse, further increasing the accuracy-speed trade-off.

C.6 A successful adaptation to the DRL paradigm of the original method developed by Dosovitskiy and Djolonga (2019), which allows for training a neural network architecture (in the context of DL-based techniques) not only on a particular cost function but on a whole distribution of them, has been provided in P.III. This contribution allows for training a DRL agent not only on a unique reward function but on a complete distribution of reward functions. At inference time, the DRL agent can be conditioned on one reward function, being able to provide several behaviors encapsulated in one unique neural network.

C.7 Open source and data have been one of the primary concerns in this work. In conjunction with every publication, media resources (e.g. online videos) have been released in order to further clarify the approaches. Furthermore, with P.II and P.III, among others, the source code and the generated datasets have been made publicly available. The DRL framework of C.4 has been opened internally for the usage of the members of the research group, and it is planned to be made publicly available soon.

\subsection{Outline}

This thesis by compendium is composed of three publications P.I - P.III, which are appended at the end. It is divided into five chapters, in the first chapter the introduction and motivation were presented, as well as the thesis objectives and contributions. The content of the remaining chapters is summarized below:

- Chapter 2 reviews the state of the art in the field of the methods and applications under research, with a special focus on classical control approaches for autonomous landing on a moving platform and autonomous UAV following, ML approaches for UAV control, DRL frameworks for robotics, synthetic data for real-world robotics, detection methods for flying UAVs and DRL methods for object detection.

- Chapter 3 introduces the methodology used in the development of the thesis and the evaluation of the results obtained. 
- Chapter 4 presents the experimental results and discussion of this thesis. The main results published in P.I - P.III are presented including the DL and DRL solutions for autonomous landing on top of a moving platform, non-cooperative UAV following, and video object detection.

- Chapter 5 presents the overall conclusions for this doctoral thesis and provides an overview of future work. 


\section{Chapter 2}

\section{Literature Review}

The present dissertation has been developed in the context of aerial robotics, with broad coverage of state-of-the-art ML techniques. On this basis, several studies in the literature provide methods and techniques which are adjacent to the present work. In the following section, the most relevant related work is included, with a special emphasis on the tangential aspects to the methods provided in the publications P.I - P.III.

\subsection{Classical Control Approaches for Autonomous UAV Land- ing and Object Following}

Although the primary aim of this work is the development and validation of ML-based techniques in the context of aerial robotics, classical control strategies are also a matter of importance for the applications under research. Indeed, classical control approaches, for autonomous UAV landing on static or moving platforms and object following, aid the evaluation of the presented methods within the scope of the related work.

Proportional-Integral-Derivative (PID) controllers are the preferred option for aggressive landing from relatively short distances Borowczyk et al. (2017); Araar et al. (2017); Wenzel et al. (2011); Hu et al. (2015); Kim et al. (2016). In Borowczyk et al. (2017), guidance and rendezvous laws which sometimes are augmented with velocity controllers for a faster approaching phase are utilized. In addition, adaptive control schema presents enhanced robustness Hu et al. (2015); Kim et al. (2016).

Trajectory optimization and control strategies are also commonly used for landing maneuvers on top of moving platforms Rucco et al. (2017); Gautam et al. (2015); Kai et al. (2015); Ling et al. (2014); Vlantis et al. (2015). In Rucco et al. (2017), the relative state of the vehicles is considered to be known. The determination of optimal rendezvous trajectories can also take wind disturbances into account. Guidance and rendezvous laws are applied in Gautam et al. (2015) and Kai et al. (2015). In Ling et al. (2014), when the desired meeting point is obtained, incorporating feedforward inputs allow for a faster response against track following errors. In Vlantis et al. (2015), a discrete-time non-linear Model Predictive Controller (MPC) which optimizes both the trajectories and the landing time was developed to address the difficult problem of landing on top of moving inclined 
platforms.

Other diverse control strategies have been utilized for the task of UAV landing on top of a moving platform. In Falanga et al. (2017), Linear-Quadratic Regulator (LQR) control is applied with polynomial feasible trajectories and visual markers. Position control with Robust and Perfect Tracking (RPT) and custom visual markers, assisted by color and shape based techniques have been utilized in Chen et al. (2016). In Macés-Hernández et al. (2017), MPC and visual markers aid the landing strategy. Only simulation results have been provided. In Lee et al. (2016), $\mathcal{L}_{1}$ adaptive control for trajectory tracking, and a vision algorithm based on color and shape are used. Image-Based Visual Servoing (IBVS) approaches are also found in the literature, assisted by visual markers Lee et al. (2012). In Rabelo et al. (2020), the landing maneuver is approached as a formation control problem. A velocity controller scheme based on jacobians and a motion capture system for real flights is employed.

In the 2017 Mohamed Bin Zayed International Robotics Challenge (MBZIRC) 1 a specific challenge for landing on top of a moving platform has taken place. Several international teams provided a solution, being time-optimal MPC the preferred option for control schema Beul et al. (2017); Baca et al. (2017); Beul et al. (2019); Bähnemann et al. (2019). Other teams opted for pure position control Cantelli et al. (2017). The detection strategy has been mostly computer vision approaches based on color and shape processing Beul et al. (2017); Baca et al. (2017); Beul et al. (2019); Bähnemann et al. (2019) and Tracking-Learning-Detection (TLD)Kalal et al. (2011) with pre-trained model for initialization Cantelli et al. (2017).

On the side of the autonomous UAV object following, classical control schemes (e.g. PID) have been applied. In Pestana et al. (2014), PID control scheme was used to control a multirotor aerial robot based on the OpenTLD Kalal et al. (2011) tracker in the image plane. Some techniques carried out specific-object detection in conjunction with continuous-tracking strategies, such as Kalman or particle filters Teuliere et al. (2011). In Teuliere et al. (2011), a color-based detector fed a particle filter that aided a pose-based PID control scheme. A nonlinear adaptive observer was used for target-object estimation and a guidance law for target-object tracking was developed in Choi and Kim (2014). In this case, the vision sensor was modeled and the complete approach was validated under numerical simulations.

Some approaches rely on an online user-defined Region-of-Interest (RoI) within the image plane during the execution of a real flight in order for the UAV to be able to follow a tracked object. In Li et al. (2016b), the authors developed a novel frequency and image-plane-domain tracker with the latter pose estimation of the object and standard PID control. In Mueller et al. (2016), a state-of-the-art tracker Hare et al. (2015) was used and a standard PID control scheme within the image plane was established. The stated work also performed real-flight validation tests and developed a novel camera handover strategy in order to enable long-term operation through several UAVs.

A specific-object detection in conjunction with continuous-tracking strategies, such as Kalman or particle filters, is applied in some techniques. In Yao et al. (2017), a strategy for human following was designed and tested in real flights and a miniature robotic blimp was used as the robotic platform, with human-pose estimation and standard PID control. In Nägeli et al. (2018), human tracking was carried out over a long period of time and long distances by means of active infrared markers and the estimation of the pose of both humans and UAVs without calibrated or stationary cameras.

Other diverse strategies have been proposed for UAV object following. IBVS has been

\footnotetext{
https://www.mbzirc.com/
} 
applied in Mondragón et al. (2011) as well as a color-based detection approach. In OlivaresMendez et al. (2011), visual fuzzy servoing has been proposed with computer vision processing based on color. Position control has been used in Koubâa and Qureshi (2018) for following low moving targets with computation over the internet (based on GPS positioning).

In this work, the landing maneuver on top of a moving platform and the object following maneuvers have been selected as the main target applications for publication P.I and P.II, respectively. They have been approached from a ML perspective in order to explore the possibilities of these novel methods.

\subsection{Machine Learning Approaches for UAV Control}

Machine learning techniques for robotics control have been increasingly studied in the last decade, partially due to the outstanding performance of deep or reinforcement learning methods in other fields (e.g. computer vision, natural language processing, etc.) Mnih et al. (2015); Krizhevsky et al. (2012); Vaswani et al. (2017). On this subject, machine learning strategies have demonstrated to be capable of properly handling complexity and non-linearities by learning an approximate function or mapping, which can be potentially useful in the context of robotics control, where complex dynamics have to be controlled for the development of precise or aggressive maneuvers.

Low-level robotics control (e.g. rotors thrust actuation for attitude control in UAVs) has been successfully approached by several methods. In Hwangbo et al. (2017), the dynamics of a multirotor UAV was precisely simulated in order to train a reinforcement learning agent for low-level attitude control. The learned policies were directly validated in the real world with a motion-capture system. In Tan et al. (2018), precise simulations and randomized system dynamics for quadruped locomotion learning with continuous states and actions were utilized for training a reinforcement learning agent. The model of a crazyflie quadrotor ${ }^{2}$ has been learned by minimizing the mean squared error with standard supervised learning in Bansal et al. (2016). The system has been controlled with LQR schema. In Koch et al. (2019), a Gazebd ${ }^{3}$ simulation has been used for training a reinforcement learning policy to control the angle velocities of a quadrotor. Trust Region Policy Optimization (TRPO) Schulman et al. (2015) and Proximal Policy Optimization (PPO) Schulman et al. (2017) DRL algorithms were utilized for training. In Hwangbo et al. (2017), the precise model parameters of a commercial quadrotor were simulated and a novel reinforcement learning algorithm was provided for attitude control. Model-based reinforcement learning was used in Lambert et al. (2019) for quadrotor attitude control in real-flights with solely on-board sensors. In Molchanov et al. (2019) multiple quadrotors of different sizes were controlled by the same policy, learned with simulated dynamics and domain randomization.

Autonomous UAV navigation through cluttered or unstructured environments has been also thoroughly studied. In Wang et al. (2018), human commands were mixed with reinforcement learning exploration-exploitation actions in order to improve safety for multirotor navigation. A Monte Carlo policy-evaluation method for vision-based navigation learning with synthetic images was utilized in Sadeghi and Levine (2016). In Kang et al. (2019a), the successful navigation for a small-sized multirotor was achieved through a combination of real-world data to learn about robot dynamics and synthetic data for vision-based collision avoidance. In Zhang et al. (2016), MPC was used to generate data at training time in

\footnotetext{
${ }^{2}$ https://www.bitcraze.io/products/old-products/crazyflie-2-0/

3 http://gazebosim.org/
} 
order to train a Deep Neural Network (DNN) policy, which was allowed to access only raw observations from the UAV on-board sensors. In testing time, the UAV was able to follow an obstacle-free trajectory even in unknown situations. The well-known Inception v3 model Szegedy et al. (2016) (pre-trained CNN) was adapted in order to enable the final layer to provide six action nodes (three transitions and three orientations). After re-training, the UAV managed to cross a room filled with a few obstacles in random locations. In Giusti et al. (2015), a DNN model was trained to map image to action probabilities (turn left, go straight or turn right) with a final softmax layer, and tested onboard by means of an Odroid-U3 processor. The performance is later compared to two automated methods and two human observers. In Sadeghi and Levine (2016), CNNs were utilized in order to map images to high-level behavior directives (e.g. turn left, turn right, rotate left, rotate right). The $\mathrm{Q}$ function was estimated through a CNN, which was trained in simulation and successfully tested in real experiments. In Kim and Chen (2015), discrete actions were directly mapped from raw images. In the stated method, the learned model is run off-board, taking advantage of a GPU in an external laptop. LIDAR-based navigation approaches have also been studied in Sampedro et al. (2018a), where Deep Deterministic Policy Gradients (DDPG) Lillicrap et al. (2015) algorithm was used to train a reinforcement learning agent in simulation to be directly deployed in the real world. In Wang et al. (2020), a sparserewards reinforcement learning setting was validated in simulation for UAV obstacle-free trajectory following. Deep Recurrent Q-Network (DQRN) has been included in Singla et al. (2019) for vision-based reinforcement learning with depth-maps. The depth-maps were generated in simulation and adapted to the real world with conditional Generative Adversarial Networks (GANs). In Wu et al. (2018), Deep Q-Network (DQN) was validated in simulation to find the shortest path to avoid an obstacle. In Greatwood and Richards (2019), reinforcement learning techniques were used to find the direction of exploration for an ArDrone 2. ${ }^{4}$ platform with MPC trajectory control. ArDrone 2.0 has been also used to find sites of interest in a simple grid disaster environment with reinforcement learning Junell et al. (2015).

Several strategies have tackled the landing maneuver on top of moving or static platforms with machine learning methods. Classical discrete reinforcement learning approaches have been used in the literature, where a Least Square Policy Iteration (LSPI) algorithm was used to land on top of a static platform in simulation Shaker et al. (2010). Both state and actions were part of a discrete space and the main sensor to estimate the state was a camera. In Ananthakrishnan et al. (2017), neural network backpropagation controllers for landing on top of a static platform have been proposed. LSPI has also been used in Vankadari et al. (2018) for the generation of velocity trajectories. Parallel Tracking and Mapping (PTAM) Klein and Murray (2007) was used for state estimation and the algorithm was trained in a Gazebo simulation. Vision-based lading with ArUco markers Romero-Ramirez et al. (2018) has also been explored Rodriguez-Ramos et al. (2018). A DDPG agent was trained in simulation, deployed in the real world and compared with a classical Position-Based Visual Servoing (PBVS) approach. In Albuquerque (2019), DDPG algorithm was utilized to train an agent in simulation and was validated in the real world by means of a motion capture system. In the stated method, several domain adaptation techniques have been tested (dynamics randomization, a universal policy with system identification, and reinforcement learning with no parameter variation).

Other approaches aim at UAV object following from different perspectives. In Sampedro et al. (2018b), a color-based detector and an image-based deep reinforcement-learning policy controller were utilized for object following with an off-the-shelf multirotor. In

${ }^{4}$ https://www.parrot.com/es/drones/parrot-ardrone-20-elite-edition 
Akhloufi et al. (2019), You Only Look Once (YOLO) v2 Redmon and Farhadi (2017) was utilized for UAV detection and discrete reinforcement learning REINFORCE [McNeela (2019) was used for learning the UAV following maneuver. A hierarchical approach that combines a model-free policy gradient method with a conventional feedback PID controller to enable stable learning without catastrophic failure was developed in Li et al. (2017) for human following.

In this thesis, DDPG and PPO algorithms were adapted to train agents in simulation to be directly deployed in the real world, for UAV landing on top of a moving platform and UAV following, respectively. Concretely, the DRL strategy for UAV landing on top of a moving platform is covered in P.I and the UAV following approach is explored in P.II.

\subsection{Reinforcement Learning Frameworks for Robotics}

The evolution of reinforcement learning methods and algorithms has been notable across the last years Lillicrap et al. (2015); Schulman et al. (2017, 2015). Indeed, most of them are commonly developed, tested, and validated on a wide variety of game-like simulated environments Brockman et al. (2016). In this regard, the research community is lacking a standard reinforcement learning framework, where robots are able to learn in a realistic simulated environment in order to be directly deployed in the real world. Nevertheless, although a standard and broadly-used reinforcement learning framework for robotics is not available for either the scientific or the industrial community, several frameworks have been proposed to this aim in the literature.

Gazebo simulator is a well-known robotics simulator widely utilized in robotics. Due to this fact, several studies aim at the generation of a reinforcement learning framework for multiple types of robots based on the stated simulator. Zamora et al. (2016) points to extending the OpenAI Gym Brockman et al. (2016) to a standard interface for the Gazebo simulator. Two default environments were provided for a custom UAV and UGV. In Nuin et al. (2019), OpenAI Gym standard interface is also adapted to the Gazebo simulator and ROS $2^{5}$ Three default environments were provided for MARA ${ }^{6}$ articulated arm. GymFC Koch et al. (2019) provides a Gazebo-based UAV simulation for low-level attitude control, with an OpenAI Gym standard interface. Ferigo et al. (2020) steps forward with the usage of the last version of the Gazebo simulator (Ignition Gazebo), which provides a simulatoras-a-library structure. Stated work provides a versatile and parallelizable simulator, with an OpenAI Gym standard interface, capable of running faster than real-time.

Other frameworks focus on photorealistic simulations for vision-based applications. The virtualization of real environments has been carried out in Xia et al. (2018) to achieve an outstanding level of realism. The stated framework is meant for embodiment robots and its physics simulation is limited. Other frameworks base their photorealistic simulations on game engines Shah et al. (2018); Juliani et al. (2018). AirSim Shah et al. (2018) is built on Unreal engine ${ }^{7}$ It is open-source, provides a Python API, and is meant for UAV and autonomous driving applications. ML-agents Juliani et al. $(2018)$ is built on Unity engine 8 . It provides a Python API and default agents, to collect observations and interact with flexible and dynamic environments. Nvidia Isaac Simulator ${ }^{9}$ is in an early-stage but already provides domain randomization, scenario management, sensor models, robot

\footnotetext{
5 https://index.ros.org/doc/ros2/

6 https://github.com/AcutronicRobotics/MARA

https://www .unrealengine.com/

$\varepsilon$ https://unity.com/

https://www.nvidia.com/en-us/deep-learning-ai/industries/robotics/
} 
models, and a whole stack of supervised and reinforcement learning algorithm support, with off-the-shelf algorithms.

Some approaches target different aspects in the context of robotics, such as scalability or low-level simulation efficiency. SURREAL framework Fan et al. (2018) defines a distributed learning framework (actors, buffer, learners, and parameters server). They developed distributed versions of reinforcement learning algorithms in order to be easily deployed in the cloud with several nodes, CPUs, and/or GPUs. It targets scalability, flexibility, and reproducibility. Deepbots Kirtas et al. (2020) is built on Webots Michel (2004) robotics simulator, it is open source and provides a standard OpenAI Gym interface. The Teaching Box Ertel et al. (2009) is an early-stage simulator based on Java and FANN library Nissen et al. (2003). RaiSim Hwangbo et al. (2018) is a simulator with an efficient contact solver which speeds up the simulation, with a private implementation. Pyrobolearn Delhaisse et al. (2020) is another framework containing both robotic environments and reinforcement learning algorithms. It is based on PyBullet Coumans and Bai (2016) and focuses on modularity and flexibility to promote code reuse.

In this thesis, a novel reinforcement learning framework for robotics has been proposed and validated across several publications, including P.I and P.II. This work has contributed to the foundation and development of the stated framework and has helped to the simplification of the prototyping and validation of reinforcement learning agents in the context of autonomous UAV applications.

\subsection{Synthetic Data for Real-World Robotics}

One of the key drawbacks in learning-based algorithms (particularly in supervised learning algorithms) is the common necessity of a notable amount of structured and annotated data for the application domain under study. On this subject, there are application cases where data within the application domain can be biased or directly not available at a considerable scale. Also, in reinforcement learning applications for robotics, where an agent is meant to safely interact with an environment a numerous amount of times, real environment interaction can become intractable for the robotic system under research. In this context, synthetic data can aid the data availability for either DL or DRL techniques. It can enable scalability and provide realistic information that can be exploited in a real-world deployment. In this trend, several approaches in the literature has explored methods for the usage of synthetic data in machine learning algorithm workflows, with the ultimate aim of being used in a real-world domain.

Low-level continuous states, such as position, velocity, or joint states, have been widely simulated based on the robot dynamics in order to train machine learning algorithms, which are ultimately validated in the real world. In Pi et al. (2020), simulated position and attitude states, based on the dynamics model of the UAV, were used to train a reinforcement learning agent to command rotors velocity for attitude control. In Hwangbo et al. (2019), a neural network was used to model the dynamics of the Anymal Hutter et al. (2016) quadrupedal-legged robot in order to learn a controller within a reinforcement learning setting. In Albuquerque (2019), domain adaptation was utilized to train a reinforcement learning agent for the task of autonomous landing on a moving platform. Relative position states, such as position and velocity states, were included during the training stage. UAV position states were simulated in Vankadari et al. (2018) for autonomous UAV landing. In stated work, fiducial markers were used in the real world to infer the position in real-time. In Hwangbo et al. (2017), AscTec Hummingbird quadro- 
tor ${ }^{10}$ was simulated with exact model parameters in order to train a reinforcement learning algorithm for attitude control. In Koch et al. (2019), IRIS quadrotor ${ }^{11}$ was simulated in a Gazebo environment for attitude velocity control with reinforcement learning. In Sampedro et al. (2018a), a quadrotor was simulated in Gazebo, along with a LIght Detection And Ranging (LIDAR) sensor, in order to train a reinforcement learning algorithm for reactive navigation. The dynamic model of a robotic manipulator has been learned in Nagabandi et al. (2020), in order to be controlled with an MPC scheme. Tan et al. (2018) utilized precise simulations and randomized system dynamics for quadruped locomotion learning with continuous states and actions. In Rodriguez-Ramos et al. (2018), high-level velocity control was learned with ground-truth simulations in order to develop a UAV task-specific landing maneuver on top of a moving platform. Validation tests were carried out through fiducial markers in the real world. In Sampedro et al. (2018b), a color-based detector and an image-based deep reinforcement-learning policy controller were utilized for object following with an off-the-shelf multirotor. In Wang et al. (2018), human commands were mixed with reinforcement-learning exploration-exploitation actions in order to improve safety for multirotor navigation. In Molchanov et al. (2019) multiple quadrotors of different sizes were controlled by the same policy, learned with simulated dynamics and domain randomization.

End-to-end robotic control based on raw synthetic images (i.e. image pixels) pose an increased challenge due to the virtual-reality domain gap in this high-dimensional space. Nevertheless, a notable amount of techniques have developed strategies to overcome the stated complexity. In Andrychowicz et al. (2020), randomization of friction coefficients and object appearance have been carried out in order to overcome the virtual-reality gap for dexterous in-hand manipulation. In Zhang et al. (2019), synthetic-data adaptation was performed using an application-dependent Cycada pipeline for ground-robot navigation. Domain-randomization techniques were used in James et al. (2019) to train a deep reinforcement-learning algorithm for dexterous in-hand manipulation. In Kang et al. (2019b), Micro Aerial Vehicle (MAV) navigation was achieved through a combination of real-world data to learn about robot dynamics and synthetic data for vision-based collision avoidance. In Rao et al. (2020), cycle GANs were utilized to transfer sim-to-real images in both directions in order to control an articulated arm.

Computer-vision techniques are tightly coupled to the robotic development, since images provide rich information which can be exploited in autonomous robotic tasks. In this context, deep learning is de facto the most used technique for object detection. Nevertheless, it usually requires a large amount of domain-specific data to achieve generalization. In this trend, the research community is exploring the incorporation of synthetic images into the training process due to their availability and capabilities. In Rajpura et al. (2017), transfer learning is used for GoogleNet Szegedy et al. (2015) training with synthetic images of common objects in a refrigerator. Gupta et al. (2016) proposed a method for the generation of synthetic text on top of images from the internet and trained a Fully Convolutional Regression Network (FCRN) with stated synthetic images. In Ros et al. (2016), a synthetic dataset is added to manually-annotated real datasets and provide an improvement on the performance for semantic segmentation. T-Net Kossaifi et al. (2019) and Fully Convolutional Network (FCN) Long et al. (2015) were used for validation. In Rozantsev et al. (2015b), feature parameters of real images for UAVs, planes, and cars were extracted and matched to the synthetic ones. This method demonstrates a higher accuracy even

\footnotetext{
${ }^{10}$ http://robotics.caltech.edu/〜ndutoit/wiki/images/7/70/AscTec_AutoPilot_manual_v1.0_ small.pdf

${ }^{11}$ http: //www .arducopter.co.uk/iris-quadcopter-uav .html
} 
when comparing to the inclusion of more photorealistic images. The stated method has been validated with a Deformable Part Model (DPM), Adaboost, and a CNN. In Simonyan and Zisserman (2014), 5M of synthetic images from indoor environments were generated for semantic segmentation (pixel perfect), object detection, instance segmentation, etc. VGG-16 Simonyan and Zisserman (2014) was pretrained on these synthetic images and outperformed the models pretrained on ImageNet Krizhevsky et al. (2012). A custom CNN was used in Zhang et al. (2018) for smoke detection with synthetic images. In Liu et al. (2020), aerial images of planes were synthetically generated and refined with Cycle GANs Zhu et al. (2017) for fine-tuning Faster R-CNN Ren et al. (2015) and R-FCN Dai et al. (2016), resulting in an accuracy improvement. In Aker and Kalkan (2017), YOLO v2 architecture was trained with synthetic images of real backgrounds overlayed with simulated birds and UAVs. Synthetic images were optimized in Rozantsev et al. (2015b) to match object-detector extracted features. These images were used for real-image dataset augmentation. In Chen et al. (2018), adversarial training was used to train Faster R-CNN for cross-domain adaptation. The domains were adapted at the image level (e.g., image style and illumination) and instance-level representation (e.g., object appearance and size shift). In Hinterstoisser et al. (2018), simple synthetic objects were detected through backbonenetwork (feature extractor) weight freezing and fine-tuning the last layers. Validation tests were performed with Faster R-CNN, R-FCN, and Mask R-CNN He et al. (2017). In Peng et al. (2015), synthetic images were generated by adding background and textures to simple 3D Computer-Aided Design (CAD) models. Features were extracted with a CNN trained on ImageNet and a classifier was trained for the final stage. In Tremblay et al. (2018), synthetic images with domain randomization were generated to train Faster R-CNN, R-FCN, and SSD Liu et al. (2016a) for common object detection. In Shrivastava et al. (2017), adversarial training was used for simulated-to-real domain transferring. The dataset was generated through synthetic-image refinement and automated labeling. Finally, novel approaches showed outstanding performance when trained with small real-world datasets, outperforming previous pretrained architectures, such as SSD or Faster R-CNN.

Additionally, object 3D-pose estimation from synthetic images has also been explored in the computer vision field. In Tobin et al. (2017), randomized simulated images for 3D object detection were composed of low-dimensional feature textures and shapes. In Rad et al. (2018), a mapping network was trained to map real to synthetic image features. The 3D pose was estimated based on the inferred object parallelogram. A dataset of synthetic heads was generated in Liu et al. (2016b) and a custom CNN was trained for head pose estimation. In Zhao et al. (2020), pairs of images with object annotation and relative transformation information between their viewpoints have been used to automatically discover objects' 3D keypoints which are geometrically and visually consistent. The $6 \mathrm{D}$ object pose has been estimated using a keypoint-based geometric reasoning method with a reference viewpoint.

In this work, a low-level simulation of a multirotor has aided the development of learning-based controllers for the task of UAV landing on top of a moving platform and object following, in publication P.I and P.II, respectively. Furthermore, in publication P.II, an object detector has been trained with supervised learning techniques, aided by transfer learning and pure synthetic images from a game-engine simulator, in order to perform the complete vision-based training with solely synthetic information.

\subsection{Detection Methods for Flying UAVs}

The increasing research on autonomous UAV systems and its successful application to diverse tasks in the industry favor the additional research and development of detection 
methods for flying UAVs. In this context, several methods for in-flight UAV detection have been found in the literature.

The majority of techniques have used images from RGB cameras for UAV detection. Schumann et al. (2017) built a UAVs and birds custom dataset. It utilized VGG-16 as the backbone and Region Proposal Network (RPN) Ren et al. (2015) as the detection header. In Gökçe et al. (2015), Haar-like features, Histogram of Gradients (HOG), and Local Binary Patterns (LBP) where used with cascades of boosted classifiers. In Li et al. (2016a), background motion estimation, moving object detection and target classification and tracking was developed for detecting UAVs in the far distance. In Ye et al. (2018), background motions via a perspective transformation model is estimated and moving object candidates are identified in the background-subtracted image through deep learning classifier trained on manually labeled datasets and motion features. In Rozantsev et al. (2016), sliding window and motion compensation is performed with two custom CNNs for object detection. In the aforementioned work, the dataset has been made publicly available. YOLO v2 architecture along with a synthetic dataset made by overlaying UAVs and birds on background images is explored in Aker and Kalkan (2017). In Unlu et al. (2018), 2D scale, rotation and translation invariant Generic Fourier Descriptor (GFD) features are used to classify targets as a UAV or bird by a neural network. Rozantsev (2017); Rozantsev et al. (2015a) proposed a machine learning technique that operates on spatiotemporal cubes of image intensities where individual patches are aligned using an object-centric regression-based motion stabilization algorithm. Adaboost and DPM detectors performed better than CNNs due to synthetic images overfitting. In Rozantsev et al. (2015b), feature parameters of real images for UAVs, planes, and cars were extracted and matched to the synthetic ones. This method demonstrates a higher accuracy even when comparing to the inclusion of more photorealistic images.

Other strategies are aided by different sources of information, such as positioning or depth images. In Opromolla et al. (2018), template matching and morphological filtering, complemented by the cooperative-UAV positioning information, is utilized for UAV detection. In Christnacher et al. (2016), acoustics, Laser Gazed Vieweing Camera (LGVC), an RGB camera, and an illumination device formed the UAV tracking task with Consensusbased Matching Tracker (CMT) Nebehay and Pflugfelder (2014). In Vrba et al. (2019), depth-images were utilized for UAV tracking in the 3D space.

In this thesis, a synthetic dataset has been generated for training a custom detector with transfer learning techniques. This study is included in P.II and the synthetic dataset has been made publicly available. Furthermore, in publication P.III a novel video object detection technique has been proposed, including the task of UAV detection. A custom dataset of in-flight UAV videos has been developed for the stated work and has been also openly released.

\subsection{Reinforcement Learning for Object Detection}

The reinforcement learning formulation and the variety of algorithms proposed in the literature allow for its application in a wide variety of settings, from video-games agent playing or robotics control to object detection methods. On this subject, there are several levels where a reinforcement learning setting can improve performance or increase efficiency.

Several reinforcement learning techniques have been applied within the single-image context for object detection. In Vassilo et al. (2020), reinforcement learning was used for choosing between an ensemble of GANs to provide higher resolution to images (operating at pixel or patch level). In Bellver et al. (2016), regions of the image which are more likely 
to contain an object were selected for object detection with reinforcement learning. In Liang et al. (2017), a semantic action graph was built using language priors for detection. Reinforcement learning agents scan the stated graph in order to find inconsistencies of context which improved accuracy. Deep reinforcement learning was utilized in Pirinen and Sminchisescu (2018) for RPN Region of Interest (RoI) selection with class-aware algorithms. In Kong et al. (2017), multi-agent reinforcement learning was leveraged for detection. Each detector is treated as an agent and they share information via Q-network to improve overall detection. Dynamic zoom-in network Gao et al. (2018) inputted a full low-resolution image and used a reinforcement learning policy to select RoI to be further processed. In Ba et al. (2014), an Long Short-Term Memory (LSTM) based model was trained with reinforcement learning to give attention to certain RoI of the image in order to find small objects. In Mathe et al. (2016); Caicedo and Lazebnik (2015) a reinforcement learning based sliding window approach found an object in a few steps. In Uzkent and Ermon (2020); Uzkent et al. (2020), an agent selected the regions of the image which need to be processed at a higher resolution or by a smaller detector to reduce computation. In Jie et al. (2016), RoIs were effectively selected based on the interdependence of objects with tree-structured reinforcement learning.

Other approaches aim at adaptive keyframe selection for video object detection. In Mahasseni et al. (2017), a policy-gradient reinforcement-learning approach made budget-aware processing by approximating the gradient of a non-decomposable and non-differentiable objective. In Liu et al. (2019a), a light policy was used for balancing the execution pipeline between an expensive and a cheap feature extractor, achieving better results as compared to a random baseline. In Luo et al. (2019), the reinforcement learning policy was guided to achieve a proper balance between detection and tracking. In Supancic III and Ramanan (2017), a learned policy was able to select when to update or to initiate a tracker.

In this work, a reinforcement learning method has been proposed in publication P.III for adaptive keyframe selection in the context of video object detection. A novel policy training technique has been developed, allowing for a reinforcement learning agent to be trained on a distribution of reward functions. The stated agent policy can be conditioned on one unique reward function at inference time, encapsulating several behaviors in one trained model. 


\section{Chapter}

\section{Methodology}

No amount of experimentation can ever prove me right; a single experiment can prove me

wrong.

Albert Einstein

As stated in Chapter 1, this thesis has been developed in the field of aerial robotics, with a special interest in the application of ML methods and techniques. On this basis, and due to the practical nature of the robotic tasks, this work is considered in the scope of Applied Research Kothari (2004). The key aspects of applied research are the following:

- Purpose The purpose of applied studies is closely associated with the solution of specific problems, in addition to the expansion of the current body of knowledge. The purpose of P.I and P.II has been to provide a solution for the task of autonomous landing on top of a moving platform and non-cooperative multirotor following, respectively, from a DRL perspective. Besides, the purpose of P.II has also been to integrate synthetic images into the standard training pipeline for object detection. Finally, the purpose of P.III has been to increase the efficiency of computation (and power) of video object detection methods, from a DL and DRL perspective.

- Context In applied studies, research objectives are mainly defined by industrial (public or private) or societal specific problems. Stated problem definition is used by public and private institutions, organizations, international competitions, etc. to define the scope of the research projects with practical and concrete goals. For each publication P.I - P.III the context application has resulted from a topic which the industry or the research community requires to be solved. In P.I, the application of multirotor landing on top of a moving platform has been thoroughly studied by the research community and it is highly interesting for the industry community (e.g. package transportation, auto-charge maneuvers, etc.). In P.II, the application of autonomous non-cooperative multirotor following compose a growing research topic and an interesting industrial topic, as long as multirotors are increasingly being used in consumer or industrial applications, and safety is required. In P.III, an efficient 
and adaptive video object detection technique composes an important topic for the context of robotics, where the temporal structure in video frames can be exploited and the algorithms require efficiency in order to be launched in an embedded system.

- Methods Research validity Pellissier (2008) represents an important element to be addressed in all types of studies. Applied studies are usually more concerned with external validity. External validity refers to the extent to which the research findings can be replicated to other environments. The methods utilized in P.I - P.III are further clarified in the rest of the present chapter.

Each publication contribution, P.I - P.III, has been framed in the scope of applied research and has complied with its stated characteristics. For every particular contribution, the purpose, context, and methods are further explained in Chapter 4

Furthermore, the methodology employed in this thesis results from a combination of a systems engineering and a software process methodology. On the one hand, SIMILAR methodology (Bahill and Gissing (1998)) is focused on the standardized definition of phases in systems engineering, which is constantly re-evaluated during their course. These phases are compatible with robotics development, which involves one of the primary objectives of this study. On the other hand, the spiral model of the software process, proposed in Boehm (1988), is oriented towards the individual properties of the software development process, with multiple phases which target the construction of a robust and stable result. This software model is particularly interesting as the present work involves the generation of software algorithms that are required to work in embedded robotic platforms and real operational environments. As a consequence, the present work follows a combination of stated methodologies and it is illustrated in Fig. 3.1.

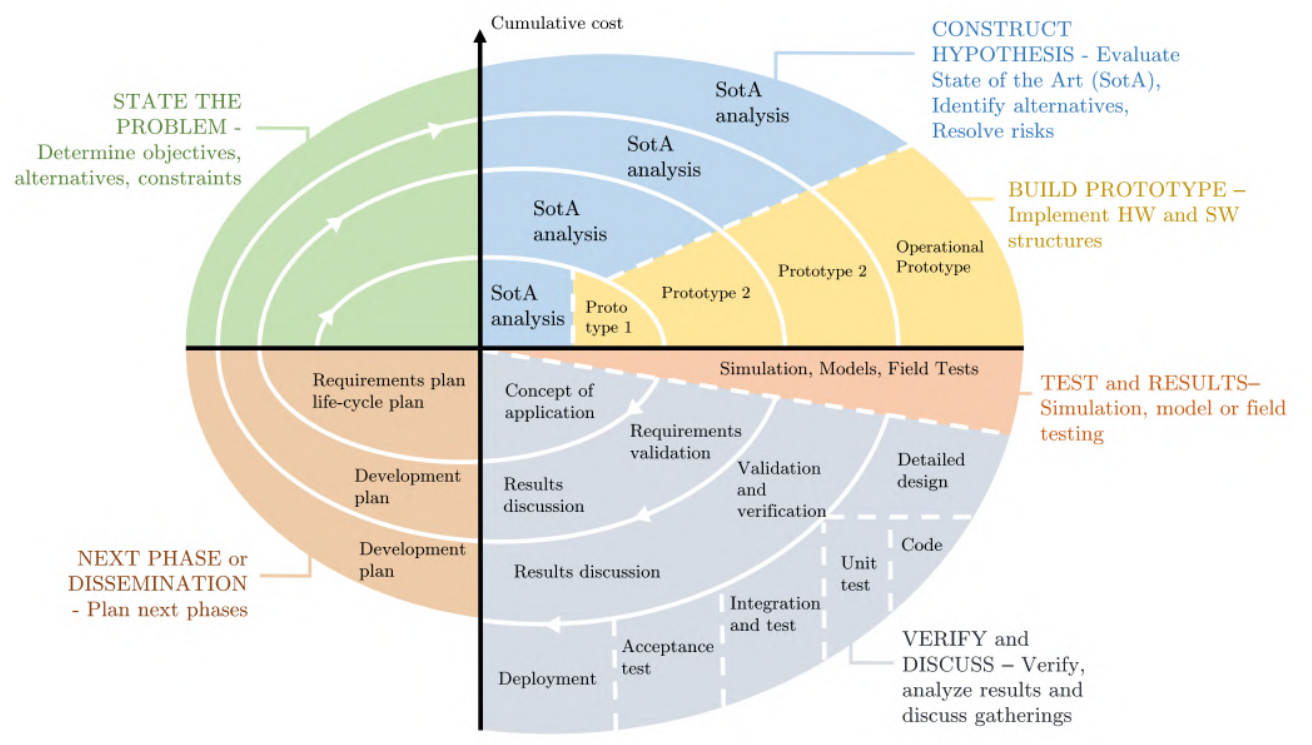

Figure 3.1: Methodology followed by the present thesis. It has resulted from a combination of SIMILAR methodology (Bahill and Gissing (1998)) and the spiral model of the software process $($ Boehm $(1988))$.

The proposed methodology has served as a general guideline during the development of this thesis, and can be summarized as a sequence of the following steps: 
1. Problem definition. The first phase involves stating the problem, which solution will potentially be provided by a future line of research. As stated at the beginning of this chapter, problems in applied research take origin from industrial or societal necessities. Once the problem has been defined, a set of objectives has to be determined in order to formally describe the solution or outcomes to be provided by the research study. During the execution of this stage, problem alternatives and context constraints have to be rigorously evaluated. In P.I, the problem has been defined as solving the multirotor landing on top of a moving platform with DRL methods. In P.II the problem has been stated as solving the non-cooperative multirotor following in an end-to-end and vision-based trend, from a DRL and DL perspective. Besides, in P.III the problem has been defined as increasing the efficiency in video object detection methods with state-of-the-art DL and DRL techniques. The global objectives, fulfilled by P.I - P.III have been defined in Chapter 1 .

2. Hypothesis formulation. In this phase, a hypothesis is formulated based on the objectives previously determined. The hypothesis has to be able to provide an outcome that is compatible with the proposed problem and objectives. During the execution of this phase, it is important to review and investigate the State of the Art (SotA) in the field in order to properly place the study in the scientific ecosystem, as well as to take advantage of the past research disseminated by the community. The alternatives found in the related works have to be evaluated and the risks involved have to be properly assessed. P.I has hypothesized that autonomous multirotor landing on top of a moving platform can be potentially solved with DRL techniques, providing a proper behavior and being able to integrate complex vision-based behaviors in an end-to-end trend. P.II has hypothesized that autonomous non-cooperative multirotor following can be potentially solved in an end-to-end and vision-based trend, integrating a high amount of DOF and solely using pure synthetic data. Finally, P.III has hypothesized that temporal structure and context information redundancy can be exploited in order to provide higher efficiency in both computation and power consumption in the context of video object detection.

3. Prototype development. This step incurs the generation of hardware and/or software components that are able to satisfy the defined objectives and comply with the requirements of the hypothesis. The resulting prototype has to be mature enough to be able to provide stable experimentation. The particular results which are being obtained in the following phases have to be taken into consideration during the execution of this stage in order to properly implement the functionality in the prototype. P.I involved the generation of both hardware and software prototypes, with the creation of stable simulated environments, a reinforcement learning framework, a real and usable moving platform, and the implementation of DRL algorithms. P.II involved the generation of stable simulated environments, the expansion of the reinforcement learning framework, the usage of an additional multirotor, and the development of the DRL and DL algorithms. P.III involved the generation of a complete software architecture for training and validation of the methods. The implemented prototypes were required to be adapted to a desktop architecture and an embedded architecture.

4. Experimentation and testing. In this stage, the prototype is thoroughly tested. A set of experiments, which has to provide the required validity for the study, is defined and carried out. Several tests are performed in order to gather a complete amount of structured data which can be analyzed in later phases. Preliminary tests usually involve simulated environments or models, while more advanced experiments 
are carried out in controlled laboratory environments or field/production domains. In P.I, thorough experimentation has been carried out in a controlled laboratory environment and with motion capture systems integrated into the approach. Also, in P.II, exhaustive experimentation has been performed in a controlled laboratory environment and using solely the on-board sensors to perform the maneuvers. In P.III, rigorous experimentation has been carried out with several model architectures, datasets, and systems.

5. Validation and verification. This phase involves the validation of the proposed hypothesis, based on the information gathered in the experimentation step. The data has to be processed in order to find usable insights, i.e. graphs, tables, metrics, etc. Also, the benefits and drawbacks of the proposed approach have to be extensively discussed to provide a rigorous outcome which verifies the original hypothesis. In P.I - P.III a complete validation and verification have been carried out, providing extensive results in the form of plots, graphs, tables, videos, etc. which were able to provide a detailed overview of the approaches.

6. Next-phase planning and/or scientific dissemination. In this final stage of the cycle, more general conclusions are assessed and the next phases are planned. As in common research studies, the results and gatherings can be shared and disseminated to the scientific community. Also, in case the findings of previous stages allow for extending the research scope, the following steps can be planned in this phase. Since every publication is related to the previous one in chronological order, the problem definition and hypothesis formulation for every publication P.I - P.III have resulted from the knowledge acquired in previous work development.

The previous set of steps composes one unique cycle of the spiral model (see Fig. 3.1). Depending on the nature of the research study or project, one or more cycle rounds are ought to be carried out. Every round in the spiral model incurs an increase of the cumulative cost, in terms of risks, time or economic resources, personnel, etc. In the context of a research study aimed for a production application, which involves a final product or service to be deployed, the proposed methodology includes extra phases to be evaluated. These phases are the detailed design of the system, extensive unit testing, integration and tests, acceptance test, and deployment and maintenance. In this thesis, P.I - P.III are applied research studies that follow the presented methodology and end with the scientific dissemination of the outcomes. Other projects in which the author has participated and are tangent to the proposed thesis study, involve final industrial application results and are described in Appendices $\mathrm{A}$ and $\mathrm{B}$.

\subsection{Technology Readiness Levels}

Additionally to the stated methodology, adopted during the course of the present thesis, Technology Readiness Levels (TRLs) have been used to evaluate the developed technologies in P.I - P.III based on their maturity. TRLs are a method for estimating the maturity of technologies during the acquisition phase of a program, developed at NASA during the 1970s Sadin et al. (1989). The method defines a measurement scale from 1-9 in order to provide a generic and standard sequence of states. In this regard, the primary purpose of using technology readiness levels is to aid management in making decisions concerning the development and transitioning of technology. Indeed, it composes one of several tools that are needed to manage the progress of research and development activities within an 
organization Deutsch et al. (2010). Using the TRLs measurement scale, the development of a technology or a system can be tracked in a wide spectrum, from an idea on a publication to a compliant product in the industry. Accordingly, TRLs are increasingly utilized by engineers, scientists, innovators, investors, etc. from many international organizations.

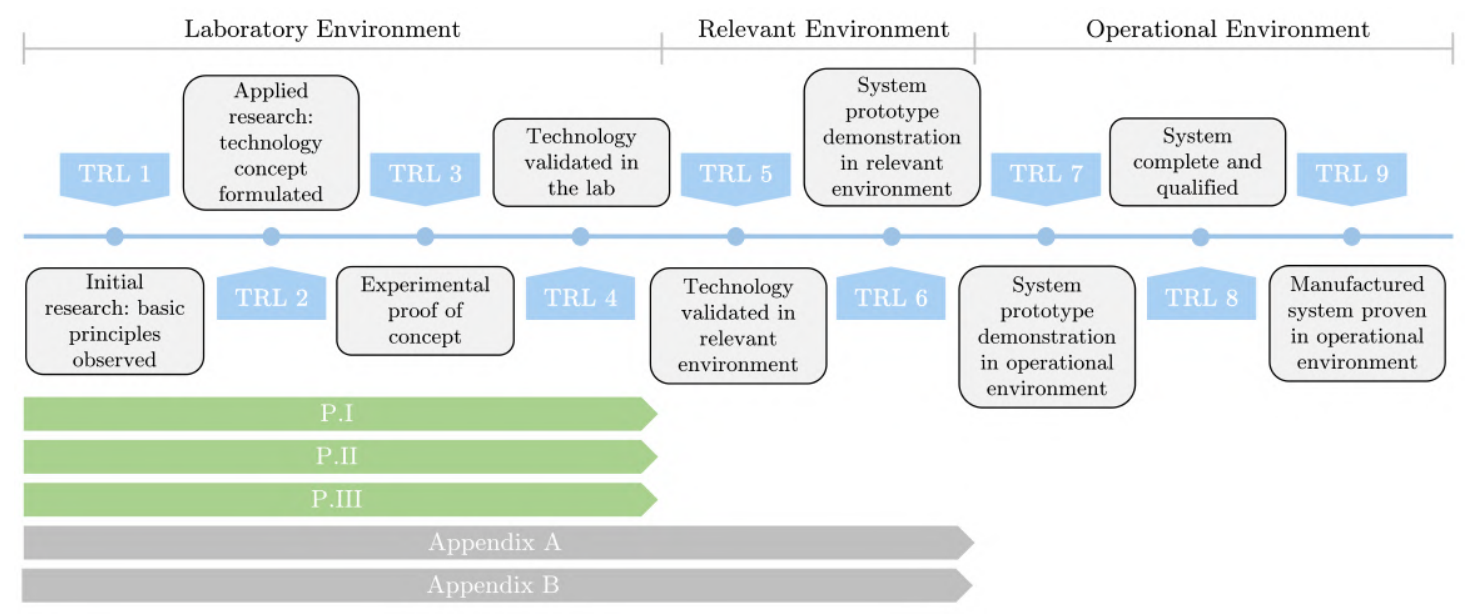

Figure 3.2: Technology readiness levels as a reference of the state of the developed technology, during the course of the presented thesis study.

In Fig. 3.2 , the different levels of technology readiness are illustrated. Also, the present work is placed within the TRL frame of reference as a standard method for benchmarking the developed research and technologies with other related scientific contexts. The terminology employed in this respect is defined as:

Laboratory environment A controlled laboratory space where the underlying principles of technical performance can be formally tested and validated. It assists the replicability and reproducibility of research but does not address all the particularities and challenges of an operational environment. Publications P.I - P.III have been tested and validated in a controlled laboratory environment, such as a robotic indoor arena or a desktop/embedded system.

Relevant environment An environment where the technologically stressing aspects and challenges of an operational environment are simulated to demonstrate the functionality of critical components of the final system.

Operational environment An environment where the final system is intended to operate. It addresses all the particularities, operational requirements, and specifications of the final system.

Besides, it has to be noted that, although TRLs provide a group of criteria which formally define the transition from one certain level to another, this thesis has not strictly followed every definition and has utilized TRLs as a maturity evaluation which helps the further assessment of the global scope of this work. A summary of the definitions and descriptions of the TRLs 1-9 can be found in Table 3.1 .

The research carried out during the course of the present study mostly involves the design or extension of innovative techniques and their application to the aerial robotics field. Due to this fact, and taking into account that this thesis is aided by state-of-the-art research within the field, publications from P.I - P.III achieve TRL 4, where the design or 


\begin{tabular}{|c|c|c|c|}
\hline TRL & Definition & Software Description & Exit Criteria \\
\hline 1 & $\begin{array}{l}\text { Basic principles observed and re- } \\
\text { ported. }\end{array}$ & $\begin{array}{l}\text { Scientific knowledge generated un- } \\
\text { derpinning basic properties of soft- } \\
\text { ware architecture and mathematical } \\
\text { formulation. }\end{array}$ & $\begin{array}{l}\text { Peer reviewed publication of re- } \\
\text { search underlying the proposed con- } \\
\text { cept/application. }\end{array}$ \\
\hline 2 & $\begin{array}{l}\text { Technology concept and/or applica- } \\
\text { tion formulated. }\end{array}$ & $\begin{array}{l}\text { Practical application is identified } \\
\text { but is speculative, no experimental } \\
\text { proof or detailed analysis is avail- } \\
\text { able to support the conjecture. Ba- } \\
\text { sic properties of algorithms, repre- } \\
\text { sentations and concepts defined. }\end{array}$ & $\begin{array}{l}\text { Documented description of the ap- } \\
\text { plication/concept that addresses } \\
\text { feasibility and benefit. }\end{array}$ \\
\hline 3 & $\begin{array}{l}\text { Analytical and experimental criti- } \\
\text { cal function and/or characteristic } \\
\text { proof of concept. }\end{array}$ & $\begin{array}{l}\text { Development of limited function- } \\
\text { ality to validate critical proper- } \\
\text { ties and predictions using non- } \\
\text { integrated software components. }\end{array}$ & $\begin{array}{l}\text { Documented analytical/experimen- } \\
\text { tal results validating predictions of } \\
\text { key parameters. }\end{array}$ \\
\hline 4 & $\begin{array}{l}\text { Component and/or breadboard val- } \\
\text { idation in laboratory environment. }\end{array}$ & $\begin{array}{l}\text { Key, functionally critical, software } \\
\text { components are integrated, and } \\
\text { functionally validated, to establish } \\
\text { interoperability and begin architec- } \\
\text { ture development. }\end{array}$ & $\begin{array}{l}\text { Documented test performance } \\
\text { demonstrating agreement with an- } \\
\text { alytical predictions. Documented } \\
\text { definition of relevant environment. }\end{array}$ \\
\hline 5 & $\begin{array}{l}\text { Component and/or breadboard val- } \\
\text { idation in relevant environment. }\end{array}$ & $\begin{array}{l}\text { End-to-end software elements } \\
\text { implemented and interfaced with } \\
\text { existing systems/simulations con- } \\
\text { forming to target environment. }\end{array}$ & $\begin{array}{l}\text { Documented test performance } \\
\text { demonstrating agreement with an- } \\
\text { alytical predictions. Documented } \\
\text { definition of scaling requirements. }\end{array}$ \\
\hline 6 & $\begin{array}{l}\text { System/sub-system model or pro- } \\
\text { totype demonstration in an opera- } \\
\text { tional environment. }\end{array}$ & $\begin{array}{l}\text { Prototype implementations of the } \\
\text { software demonstrated on full-scale } \\
\text { realistic problems. Partially inte- } \\
\text { grate with existing hardware/soft- } \\
\text { ware systems. }\end{array}$ & $\begin{array}{l}\text { Documented test performance } \\
\text { demonstrating agreement with } \\
\text { analytical predictions. }\end{array}$ \\
\hline 7 & $\begin{array}{l}\text { System prototype demonstration in } \\
\text { an operational environment. }\end{array}$ & $\begin{array}{l}\text { Prototype software exists having } \\
\text { all key functionality available for } \\
\text { demonstration and test. Well inte- } \\
\text { grated with operational hardware/- } \\
\text { software systems demonstrating op- } \\
\text { erational feasibility. }\end{array}$ & $\begin{array}{l}\text { Documented test performance } \\
\text { demonstrating agreement with } \\
\text { analytical predictions. }\end{array}$ \\
\hline 8 & $\begin{array}{l}\text { Actual system completed and 'flight } \\
\text { qualified' through test and demon- } \\
\text { stration. }\end{array}$ & $\begin{array}{l}\text { All software has been thoroughly } \\
\text { debugged and fully integrated with } \\
\text { all operational hardware and soft- } \\
\text { ware systems. All user documenta- } \\
\text { tion, training documentation, and } \\
\text { maintenance documentation com- } \\
\text { pleted. }\end{array}$ & $\begin{array}{l}\text { Documented test performance veri- } \\
\text { fying analytical predictions. }\end{array}$ \\
\hline 9 & $\begin{array}{l}\text { Actual system flight proven through } \\
\text { successful mission operations. }\end{array}$ & $\begin{array}{l}\text { All software has been thoroughly } \\
\text { debugged and fully integrated with } \\
\text { all operational hardware/software } \\
\text { systems. All documentation has } \\
\text { been completed. }\end{array}$ & $\begin{array}{l}\text { Documented mission } \\
\text { results. }\end{array}$ \\
\hline
\end{tabular}

Table 3.1: Technology readiness levels table of definitions.

extension of a particular method and its application on a robotic platform in a controlled laboratory environment composes itself a contribution to the field. Furthermore, the author of this thesis has participated in several adjacent industrial projects and international competitions. The most relevant ones are summarized in Appendix $\mathrm{A}$ and $\mathrm{B}$, and achieve TRL 6, incurring a successful system demonstration in a relevant environment, such as an industrial or competition environment. 


\section{Chapter}

\section{Results and Discussion}

This chapter presents a description of the approaches proposed in publications P.I - P.III towards achieving the research objectives of this doctoral thesis, presented in Section 1.3 . Additionally, general analysis and discussion of the results obtained in the experimental tests performed are provided. The main contributions C.1 - C.7 are listed in Section 1.4 Whether any added information is required, the reader is encouraged to consult the published works P.I - P.III which are included as part of this thesis by compendium, as well as the complementary publications which have been briefly explained in this dissertation. As a visual summary, Fig. 4.1 presents a flow chart of the interconnections and dependencies between the publications.

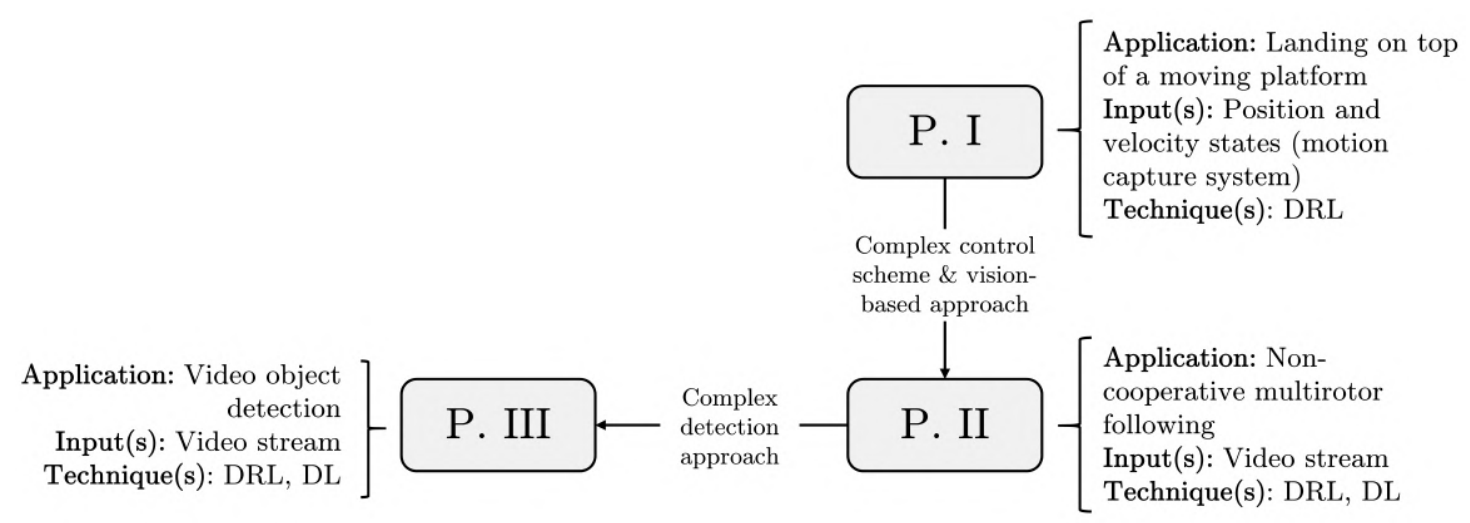

Figure 4.1: Flow chart of the publications included in this thesis by compendium. The key differentiators and interconnections have been summarized.

Publications P.I - P.III have followed a chronological order. The knowledge and experience acquired from a certain publication were consequently applied tod the following ones. Indeed, this is further explained in the following sections. However, Fig. 4.1 can serve as a schematic summary which can be revised at any time. On this basis, P.I laid the foundations for the methods to perform aerial-robot velocity control with DRL techniques, for the specific application of autonomous landing on a moving platform. The approach was based on low-level continuous states, such as position and velocity states and it was 
validated with a motion capture system. Following this idea, and in order to avoid the necessity of motion capture systems, as well as to pursue the usage of end-to-end imagebased techniques, P.II explored the possibility of controlling a UAV with DRL techniques in a complex scenario for the task of non-cooperative UAV following. In this particular case, the complete approach was based on the images from an RGB camera, the control was carried out at a lower level (attitude control) and there were 5-DOF involved (3-DOF of the UAV and 2-DOF of the camera gimbal). Additionally, the complete approach was carried out with synthetic information, and a novel technique for object detection, aided by synthetic images from a game-engine, was proposed. The stated detection technique, albeit useful in the context of P.II application, was not exploiting the temporal information found in standard video sequences. In this trend, P.III aimed at taking into consideration techniques to adaptively reuse information in video sequences, in order to reduce the computation overhead. Furthermore, a novel strategy was presented in P.III, where a DRL agent can be trained with a distribution of reward functions, and, at inference time, it can be conditioned on one unique reward function, encapsulating several behaviors in one unique policy.

The rest of this chapter is divided as follows. First, Section 4.1 introduces general concepts that are part of the global solutions developed. Second, Section 4.2 details the proposed reinforcement learning framework, with explanations of the design, structure, and scope. Finally, Section 4.3, Section 4.4, and Section 4.5 describe and discuss the results corresponding to P.I, P.II, and P.III approaches, respectively.

\subsection{Overall Concepts}

A brief introduction about overall concepts, such as robot dynamics or sensory utilization, is detailed in this section. In this doctoral thesis, although the control techniques are performed at a middle or high level (i.e. attitude or velocity control), it is useful, for the sake of understanding, to describe the dynamics of the system and the common control schemes, utilized in P.I - P.III. The base UAV system has been a multirotor with four rotors (quadrotor) distributed in "X" shape (see Fig. 4.2). The quadrotor rigid-body dynamics model has been extensively explained in multiple studies Michael et al. (2010); Hoffmann et al. (2008) and can be easily inferred from physical laws:

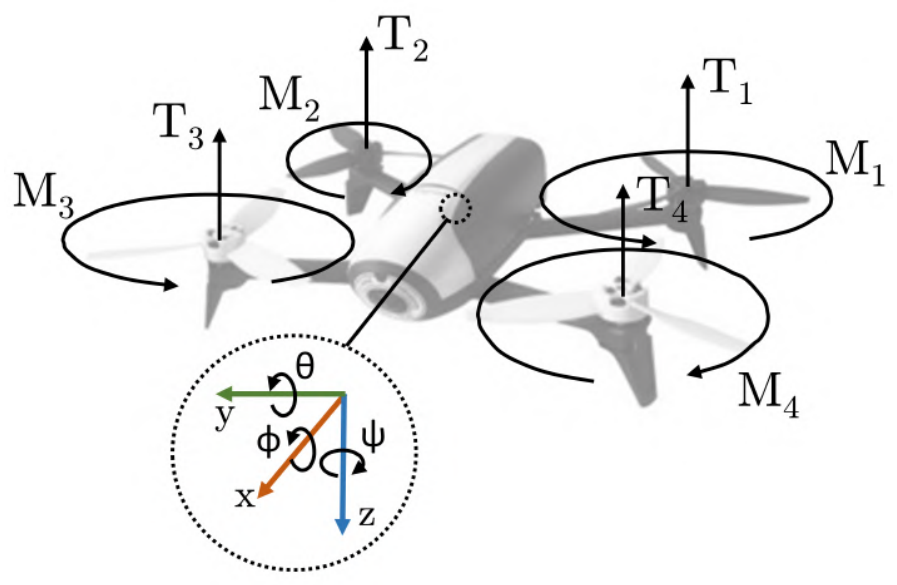

Figure 4.2: The base UAV system with four rotors (quadrotor) distributed in "X" shape. $T_{i}$ corresponds to the $i$-th thrust and $M_{i}$ to the $i$-th heading torque. 


$$
\begin{aligned}
I_{x} \ddot{\phi} & =\dot{\psi} \dot{\theta}\left(I_{y}-I_{z}\right)+l\left(T_{1}+T_{2}-T_{3}-T_{4}\right) \\
I_{y} \ddot{\theta} & =\dot{\phi} \dot{\psi}\left(I_{z}-I_{x}\right)+l\left(T_{1}+T_{4}-T_{2}-T_{3}\right) \\
I_{z} \ddot{\psi} & =\dot{\theta} \dot{\phi}\left(I_{x}-I_{y}\right)+\sum_{i=1}^{4} M_{i} \\
m \ddot{x} & =(-\sin \phi \sin \psi-\cos \phi \sin \theta \cos \psi) \\
& \times \sum_{i=1}^{4} T_{i}-K_{f r} \dot{x}^{2} \\
m \ddot{y} & =(-\cos \phi \sin \theta \sin \psi+\sin \phi \cos \psi) \\
& \times \sum_{i=1}^{4} T_{i}-K_{f r} \dot{y}^{2} \\
m \ddot{z} & =m g-\cos \theta \cos \phi \sum_{i=1}^{4} T_{i}
\end{aligned}
$$

where $\mathrm{x}, \mathrm{y}$, and $\mathrm{z}$ are the positions of the quadrotor in every axis of the world frame; $\psi$, $\theta$ and $\phi$ are the attitude angles yaw, pitch, and roll, respectively; the quadrotor rigid body is characterized by its mass $m$ and its three principal mass moments of inertia $I_{x}, I_{y}$, and $I_{z}$, each propeller $i$ generates a thrust $T_{i}$ and a heading torque $M_{i} ; K_{f r}$ is an aerodynamic friction constant and $l$ constant is the arm length between each pair of trusts.

There are two control loops which are usually implemented in the autopilot board of a multirotor. These are the attitude $(\psi, \theta$, and $\phi$ or $\dot{\psi}, \dot{\theta}$, and $\dot{\phi})$ and altitude $\left(T_{z} / \dot{z}\right)$ control loops. The autopilot boards usually accept yaw, pitch and roll absolute or rate references, and $\mathrm{z}$ absolute thrust or altitude rate references. In this scenario, the saturation bounds for these variables are set by the autopilot board design. In Fig. 4.3 a common cascaded control architecture is illustrated, with a special emphasis on the scope for publication P.I and P.II.

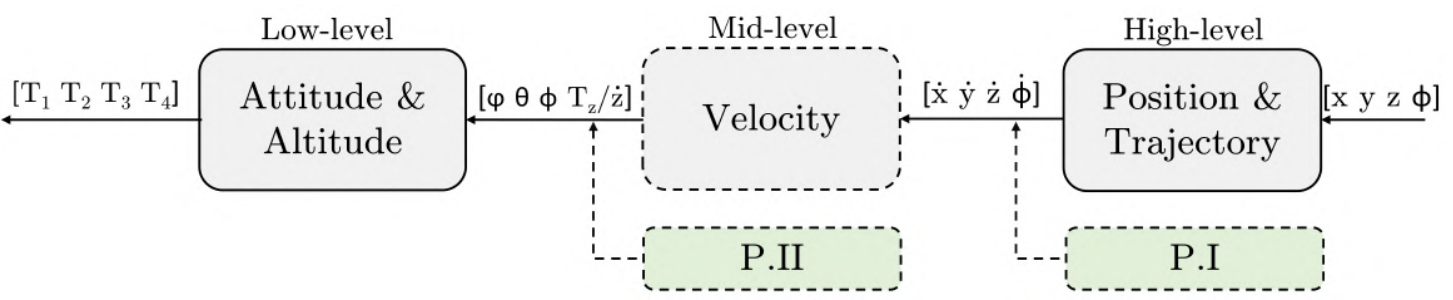

Figure 4.3: Basic cascaded control scheme for multirotor control. The publications P.I and P.II have been framed in the scope of velocity and attitude control, respectively.

The mid-level control loop is frequently a velocity control scheme and the high-level position or trajectory control loop is in charge of actuating on the mid-level velocity (or directly on the low-level attitude control loop). The magnitude of the aggressiveness, in terms of maximum accelerations and velocities which the multirotor can achieve in a controlled trend, is limited by the lower levels of control. High acceleration and velocity maneuvers are best controlled at a lower level, at the expense of increased complexity due to faster effective dynamics and higher control and state-estimation frequencies. In P.I, high-level velocity references were generated, and in P.II, mid-level attitude references were produced. 
Regarding state estimation for control, several approaches are depending on which sensors are integrated into the multirotor platform. In this dissertation, one of the primary aims is to research techniques for vision-based autonomous multirotor applications. Hence, the developed techniques have been targeted at the usage of information data from RGB cameras and Inertial Measurement Units (IMUs). Images from RGB camera sensors provide high-dimensional information that can be useful in several environments but, at the same time, can complicate the extraction of tractable data for a given stage of the maneuver or mission. Other sensors, such as LIDAR sensors or stereo cameras, have been explored in Appendix $\mathrm{A}$ and $\mathrm{B}$.

\subsection{Reinforcement Learning Framework}

In this section, the proposed reinforcement learning framework is detailed. This section is in accordance with C.4, and fulfills objectives $\mathbf{O . 2}$ and $\mathbf{O . 6}$.

\subsubsection{Introduction}

In reinforcement learning, two basic structures are defined in its formulation: an agent and an environment. The agent interacts with an environment in order to find the best possible action at any given state, and it is signalized about its performance with a reward, as illustrated in Fig. 4.4. The agent must also find a proper balance between exploration and exploitation of the given state-space knowledge. On the course of the stated interaction, the agent modifies its behavior or policy to maximize the accumulated reward over time. Indeed, reinforcement learning involves a policy optimization process throughout the whole known state space, in order to maximize the accumulated reward. For the robotics case, which is normally task-based and with temporal structure, the reinforcement learning formulation is suitable to optimize a given task or application.

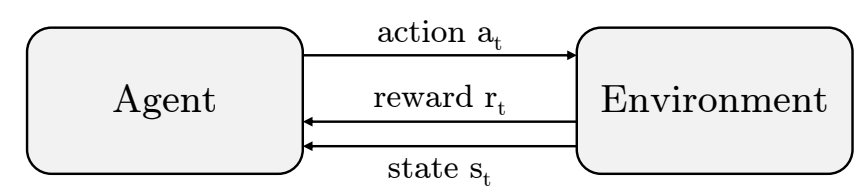

Figure 4.4: Reinforcement learning problem formulation. The basic structures are the agent and the environment. The agent provide actions $a_{t}$ to the environment which responds with the next state $s_{t}$ and the reward $r_{t}$.

In the standard reinforcement learning theory, an agent follows a policy which is able to map any give state $s \in \mathbb{S}$ to an action $a \in \mathbb{A}$, where $\mathbb{S}$ is the state space (possible states of the agent in the environment) and $\mathbb{A}$ is the action space. The inner dynamics of the environment are represented by the transition probability model $p\left(s_{t+1} \mid s_{t}, a_{t}\right)$ at time $t$. The policy can be stochastic $\pi(a \mid s)$, with a probability associated with each possible action, or deterministic $\pi(s)$. In each time step, the policy determines the action to be chosen and the reward $r\left(s_{t}, a_{t}\right)$ is observed from the environment. The goal of the agent is to maximize the accumulated discounted reward $R_{t}=\sum_{i=t}^{T} \gamma^{i-t} r\left(s_{i}, a_{i}\right)$ from a state at time $t$ to time $T$ ( $T=\infty$ for infinite horizon problems) Sutton and Barto (2018). The discount factor $\gamma$ is defined to allocate different weights for future rewards.

For a specific policy $\pi$, the value function $V^{\pi}$ in 4.2 is a representation of the expecta- 
tion of the accumulated discounted reward $R_{t}$ for each state $s \in \mathbb{S}$ (assuming a deterministic policy $\left.\pi\left(s_{t}\right)\right)$.

$$
V^{\pi}\left(s_{t}\right)=\mathbb{E}\left[R_{t} \mid s_{t}, a_{t}=\pi\left(s_{t}\right)\right]
$$

An equivalent of the value function is represented by the action-value function $Q^{\pi}$ in 4.3 for every action-state pair $\left(s_{t}, a_{t}\right)$.

$$
Q^{\pi}\left(s_{t}, a_{t}\right)=r\left(s_{t}, a_{t}\right)+\gamma \sum_{s_{t+1}} p\left(s_{t+1} \mid s_{t}, a_{t}\right) V^{\pi}\left(s_{t+1}\right)
$$

The optimal policy $\pi^{*}$ shall be the one that maximizes the value function (or equivalently the action-value function), as in (4.4).

$$
\pi^{*}=\underset{\pi}{\arg \max } V^{\pi}\left(s_{t}\right)=\underset{a_{t}}{\arg \max } Q^{*}\left(s_{t}, a_{t}\right)
$$

A general case in real robotic applications is that the state and action spaces are continuous spaces (e.g. continuous control of the rotors of a multirotor based on angular states), or the number of possible discrete states is notably high (e.g. possible values of the pixels in an image). These scenarios of high dimensionality can make the optimization problem intractable. These and other challenges are on the focus of several types of research on innovative reinforcement learning algorithms. Additionally, the formulation of an algorithm for a specific application composes a challenge itself due to stability or policy optimization non-optimal results.

\subsubsection{Deep Deterministic Policy Gradients}

In the context of state-of-the-art DRL algorithms, Deep Deterministic Policy Gradients (DDPG) Lillicrap et al. (2015) represents one of the first successful applications of neural networks for function estimation to the reinforcement learning paradigm. DDPG is a policy-gradient DRL algorithm designed to work with both continuous state and action spaces. Policy-gradient reinforcement learning methods aim towards directly searching the optimal policy $\pi^{*}$, which provides a feasible framework for continuous control. If the target policy $\pi^{*}$ is a deterministic policy $\mu$, the Q function (see 4.5) can be learned offpolicy, using transitions (from an environment $E$ ) which are generated from a different stochastic behavior policy $\beta$ Lillicrap et al. (2015). An advantage of the off-policy methods is that exploration can be treated independently from learning. In this case, exploration is carried out throughout autocorrelated Ornstein-Uhlenbeck exploration noise Uhlenbeck and Ornstein (1930).

Prior to the design of DDPG algorithm, the key factors which led to the convergence of the optimization process with large neural networks for estimation were the use of a replay buffer, and a separate target network for calculating $y_{t}$, as firstly proven by Deep Q-Network Mnih et al. (2013). In order to deal with large continuous state and action spaces, DDPG adapted the actor-critic paradigm introduced in Silver et al. (2014), with two neural networks to approximate a greedy deterministic policy $\mu$ (actor) and the $Q$ function (critic). In DDPG, the $Q$ function is estimated as in (4.5).

$$
Q\left(s_{t}, a_{t}\right)=\mathbb{E}_{r_{t}, s_{t+1} \sim E}\left[r\left(s_{t}, a_{t}\right)+\gamma Q^{\mu}\left(s_{t+1}, \mu\left(s_{t+1}\right)\right)\right]
$$

A function approximator (in this case a neural network), parameterized by $\theta^{Q}$, is considered in DDPG to approximate the $\mathrm{Q}$ function. It is optimized by minimizing the loss 
$L\left(\theta^{Q}\right)$ of 4.6 .

$$
L\left(\theta^{Q}\right)=\mathbb{E}_{s_{t} \sim \rho^{\beta}, a_{t} \sim \beta, r_{t} \sim E}\left[\left(Q\left(s_{t}, a_{t} \mid \theta^{Q}\right)-y_{t}\right)^{2}\right]
$$

where

$$
y_{t}=r\left(s_{t}, a_{t}\right)+\gamma Q\left(s_{t+1}, \mu\left(s_{t+1}\right) \mid \theta^{Q}\right)
$$

The actor network is updated by following and applying the chain rule to the expected return from the start distribution $J$ concerning the actor parameters (see 4.8).

$$
\nabla_{\theta \mu} J \approx \mathbb{E}_{s_{t} \sim \rho^{\beta}}\left[\left.\nabla_{\theta \mu} Q\left(s, a \mid \theta^{Q}\right)\right|_{s=s_{t}, a=\mu\left(s_{t} \mid \theta^{\mu}\right)}\right]
$$

An adaptation of DDPG algorithm has been mainly utilized in P.I. In P.II, DDPG has been also validated for the application under study, though provided a lower performance with respect to other DRL algorithms.

\subsubsection{Proximal Policy Optimization}

Other recent algorithms, which compose a family of natural policy-gradient algorithms named Proximal Policy Optimization (PPO) Schulman et al. (2017), include a surrogate objective function for optimization. This family of algorithms led to a reduction of complexity and an increase in both sample efficiency and performance. Since PPO aims at directly optimizing an objective function for the policy, an instant update of the policy has to be controlled in order to avoid divergence and to assure optimal convergence. The strategies for constraining the policy update are diverse and a matter of research. Nevertheless, the most common strategies are based on the Kullback-Leibler divergence (KL-divergence) penalty and on direct-clipping penalty (see 4.9p).

$$
\begin{aligned}
r_{t}(\theta) & =\frac{\pi_{\theta}\left(a_{t} \mid s_{t}\right)}{\pi_{\theta_{\text {old }}}\left(a_{t} \mid s_{t}\right)} \\
\mathcal{L}_{\theta_{k}}^{C L I P} & =\mathbb{E}_{\tau \sim \pi_{k}}\left[\sum_{t=0}^{T}\left[\min \left(r_{t}(\theta) \hat{A}_{t}^{\pi_{k}}, \operatorname{clip}\left(r_{t}(\theta), 1-\epsilon, 1+\epsilon\right) \hat{A}_{t}^{\pi_{k}}\right)\right]\right]
\end{aligned}
$$

where $\mathcal{L}_{\theta_{k}}^{C L I P}$ represents the objective function for the policy-weight update, $r_{t}(\theta)$ is the new-old policy ratio, $\hat{A}_{t}^{\pi_{k}}$ is the advantage function for policy update at time $t$, and $\epsilon$ is the clipping constraint. The advantage function is constructed based on the difference between the expected reward and the estimation of a baseline $Q(s, a)$ or $V(s)$. Indeed, the usage of an advantage function, instead of directly the expected reward, reduces the variance of the policy estimation. An adaptation of PPO algorithm has been utilized in P.II and P.III.

\subsubsection{Framework Design}

Traditional reinforcement learning algorithms Sutton and Barto (2018) have been tested and validated by means of generic tools for simulation and modeling (e.g. Matlab 1 , Simulink). On the side of these classic reinforcement learning algorithms, their performance was not powerful enough to overcome high-level and complex problems, neither in the context of continuous states and action spaces nor working with continuous physics in realistic simulators (e.g. Gazebo simulator ${ }^{2}$. Nevertheless, as explained in this chapter,

\footnotetext{
${ }^{1}$ https://www.mathworks.com/products/matlab.html

2 http://gazebosim.org/
} 
increasing improvement in the stated DRL algorithms has taken place. In a similar trend, simulation systems have been following this complexity trend, allowing a feasible and stable training of DRL agents under realistic and continuous simulators.

In addition, as explained in Chapter 2, several attempts for the generation of successful DRL frameworks for robotics have been investigated in parallel to the development of the present thesis work. In this regard, one of the first and most extended test benches for DRL algorithms is OpenAI Gym, which is an open-source toolkit for developing and comparing reinforcement learning algorithms Brockman et al. (2016). However, currently most of the available environments for testing in OpenAI Gym are 2D or Atari-like games, with a diverse range of complexity examples, but lacking more realistic environments for robotic simulation. In robotics, this type of training/testing environments are not directly available for the research community or target different aspects of the robotic development (refer to Section 2.3.

On the other hand, the Gazebo simulator is often a prime example of a versatile and realistic simulation system for robotics, normally aided by the well-known Robot Operating System (ROS) middleware Quigley et al. (2009). Hence, taking advantage of the versatility of this broadly-used simulation system, a Gazebo-based reinforcement learning framework has been designed and implemented. This framework aims to provide a predefined interface between the DRL algorithm, the environment interface, and the Gazebo simulator (or other types of simulators). Also, it has been designed in such a way that it can be extended in the future to other types of DRL algorithms, environments, and robots.

As stated in Section 4.2.1, in reinforcement learning, an agent interacts with an environment and tries to maximize the accumulated reward in each time step. In the proposed framework, the communication channel between the agent and the environment is implemented through commonly-used ROS communication tools, which provide a standardized way of communication (see Fig. 4.5). Also, some reinforcement learning problems require the simulation time to be deliberately iterated (with the simulation clock in the paused state), in order to complete computationally-expensive training steps. In this scenario, where the Gazebo simulator clock is paused, communication via ROS tools is not possible, since ROS uses the Gazebo clock to forward its messages through the network. In order to overcome these issues, an additional shared memory communication channel has been implemented, which can be used in case the Gazebo clock is paused and the simulation is being actively iterated. This functionality provides the developer with full control over the simulation time, allowing the interaction of a Gazebo plugin with the Gazebo simulator and the shared memory interface, which can be a requirement in several real-world problems.

Furthermore, the agent component which represents the classical reinforcement learning agent is capable of receiving experience vectors and rewards from the environment in order to find the optimum action to be taken in each time step. The implementation of the agent is also capable of logging representative data which can be used to determine whether it is actually learning or whether the algorithm has otherwise diverged. The current framework implementation provides DDPG, PPO, TRPO, Actor-Critic with Experimence Replay (ACER) Wang et al. (2016), and other state-of-the-art algorithms to be directly trained/tested in the scope of the designed application case. On the other hand, the environment interface shown in Fig. 4.5 represents a partial implementation of the classical definition of a reinforcement-learning environment since in this case, the Gazebo simulator and Aerostack architecture Sanchez-Lopez et al. (2016) constitute an important part of the whole reinforcement learning environment. Aerostack is a system architecture and open-source multi-purpose software framework for autonomous multi-UAV operation. In this dissertation, it has been used to enable the operation of the UAVs in both training 


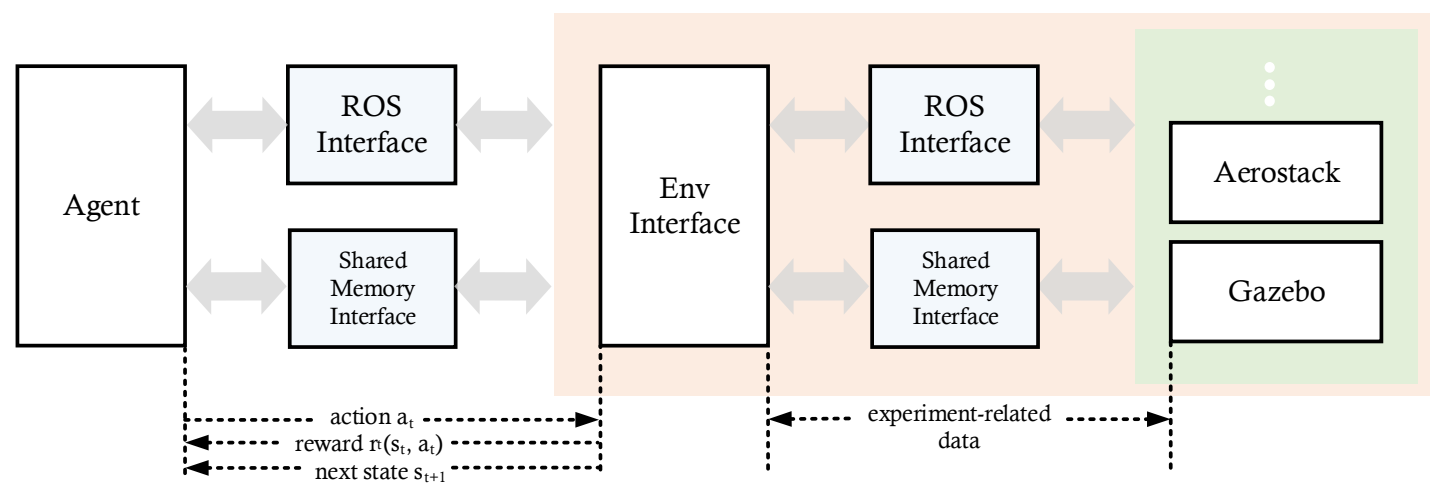

Figure 4.5: Diagram of the proposed reinforcement learning framework. The theoretical concept of the reinforcement learning environment is highlighted in pale red. Simulation systems or multi-purpose architectures are highlighted in pale green.

and testing time, though its full explanation is out of the scope of this work. The reader is encouraged to revise Sanchez-Lopez et al. (2016) for further information.

Additionally, the environment interface shown in Fig. 4.5 implements an interface between Gazebo/Aerostack and the agent, being in charge of parsing all the incoming data, in order to adapt it to an intelligible structure which the agent can use. The environment interface is ready to provide both low-dimensional discrete or continuous state vectors, as well as other types of structures such as images or point clouds. Furthermore, taking into consideration future extensions of either agents, environments, robots, or simulation systems, the framework has been designed in a versatile manner at a programming level. The current implementation utilizes Python for the agent and $\mathrm{C}++$ for the environment and communications side. Nevertheless, since all the communication interfaces are standard and cross-language, both the agent and the environment interface can be implemented in a wide variety of programming languages, such as $\mathrm{C}++$, Python, or Java.

Finally, the presented framework is designed to be used with Gazebo, but it can be adapted to any other simulation systems (as well as simulated robots), due to the standard nature of its communications. Also, the simulation time can be speeded up or slowed down, in order to reduce training times and to adapt the simulation to computationally-expensive experiments, respectively. This framework has been constantly growing since its original publication in P.I and Sampedro et al. (2019), and has contributed to the development of several other publications Rodriguez-Ramos et al. (2018); Sampedro et al. (2018b a), P.II, a Ph.D. thesis Pérez (2019) and other M.Sc. thesis Zafra (2020).

\subsection{Autonomous Landing on a Moving Platform (Publication P.I)}

In publication P.I, the autonomous landing maneuver on top of a moving platform has been approached from a DRL perspective. This section explains the most relevant aspects of the P.I approach and its outcomes, as well as the classical methods which laid the foundation of the aforementioned publication. Also, this section is in accordance with C.1, C.3 - C.4, and C.7; and fulfills objectives O.1 - O.2, O.4 and O.5 - O.6. 


\subsubsection{Introduction}

Several studies have proven the potential usefulness of autonomous multirotor systems in a wide variety of applications, such as search-and-rescue, inspection, transportation, mapping, or surveillance Liu et al. (2014); Goodrich et al. (2009). Indeed, many of these applications can greatly benefit from aerial robots capable of landing on predefined static or moving platforms. For instance, in a future where aerial robots perform search-andrescue or package transportation missions, a static or moving ground-station can be ready for supply/package delivery and/or battery charging. This would represent a major step forward which can enable the explosion of a wide range of long-term autonomous multirotor applications.

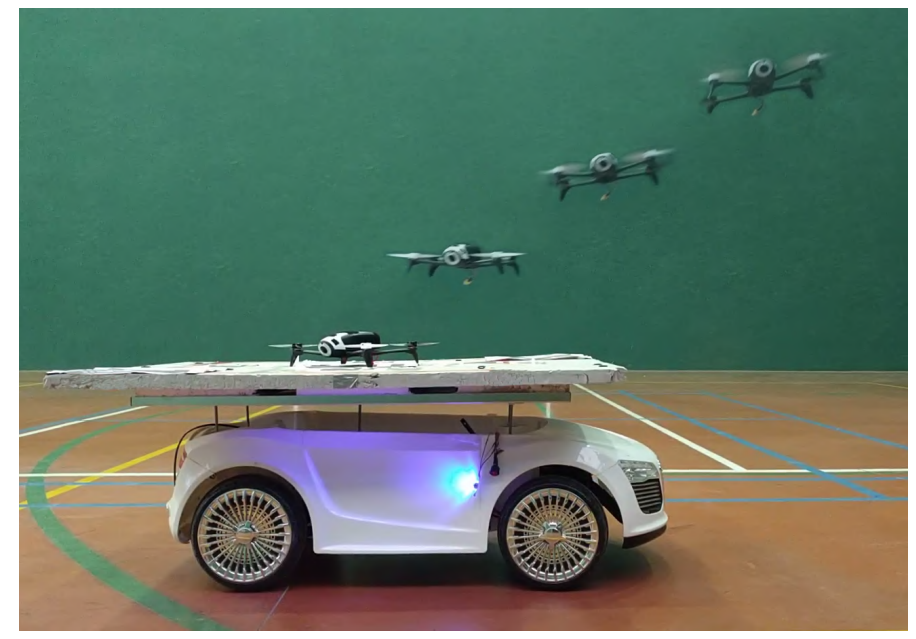

Figure 4.6: A real image composition of a multirotor landing on a moving platform using Rodriguez-Ramos et al. (2018) approach.

The landing maneuver on top of a Moving Platform (MP) can be approached from several perspectives. Depending on the available sensory information, the design can either assume the state of the MP as known or, on the contrary, assume that only information from proprioceptive and passive sensors, such as IMUs and RGB cameras, is available during the performance. In addition, the solution can be aided by classical control schemes or learning-based architectures. This dissertation has explored several approaches, from classical techniques based on 1D-LIDAR sensors, to more advanced vision-based strategies using DRL, which are best explained in the following sections. Nevertheless, the ultimate goal of this section is to validate the vision and DRL based strategies are capable of solving the landing application, with P.I as the primary contributor to this aim.

\subsubsection{Classical Methods}

Different classical approaches have been explored in the literature for autonomous multirotor landing on a MP (see Section 2.1). In this thesis by compendium, two classical techniques have been explored Rodriguez-Ramos et al. (2017, 2018). These studies accumulated enough knowledge, regarding the challenges and requirements for the application under study, and finally led to the developed DRL strategies, included in P.I and Rodriguez-Ramos et al. (2018).

On the one hand, in Rodriguez-Ramos et al. (2017), the MP was assumed to be moving in a $2 \mathrm{D}$ rectilinear trajectory. The approach also assumed that one dimension of an arbitrary point of the trajectory was known at testing time. The multirotor was able to reach 
the stated arbitrary point of the rectilinear trajectory and to predict the precise moment to perform the landing maneuver. The proposed technique was based on three stages (see Fig. 4.7): estimation (Helipad Detector, Image-based Pose Extractor, Altimeter-based Velocity Extractor, Flight Altitude Estimator and Motion Estimator), prediction (Motion Predictor), and fast landing (Fast Landing).

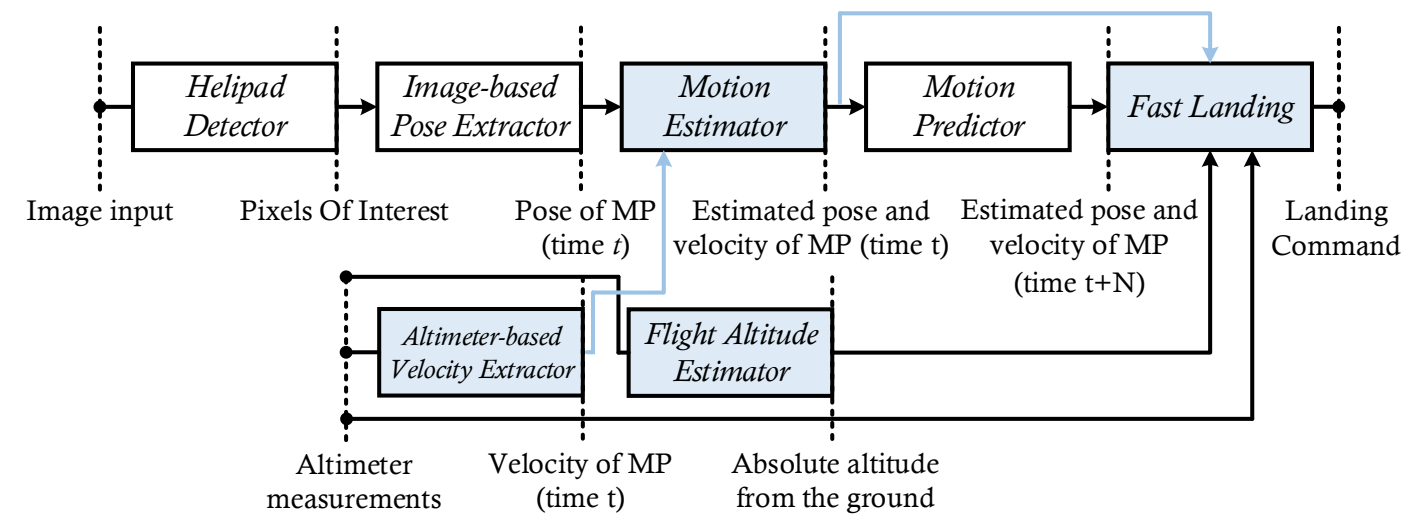

Figure 4.7: All the components involved in the Rodriguez-Ramos et al. (2017) approach: estimation (Helipad Detector, Image-based Pose Extractor, Altimeter-based Velocity Extractor, Flight Altitude Estimator and Motion Estimator), prediction (Motion Predictor) and fast landing (Fast Landing). The preliminary components developed for the IMAV 2016 competition are highlighted in light blue.

In the estimation stage, the multirotor is awaiting at a high altitude (hovering) and forcing the MP to be within its field of view, in order to estimate the motion of the MP (pose and velocity). In the prediction stage, the UAV is able to predict the motion of the MP in a predefined range of space. The prediction range will depend on the prediction algorithm capabilities, the maximum velocity of the multirotor, and the randomness of the current trajectory. The aim is to have high confidence about a certain position and velocity of the MP in a future instant of time. After the estimation and prediction stages have converged, the multirotor proceeds to the fast landing stage. The multirotor moves to the predicted position of the MP, at a safe altitude to land. In that stage, utilizing the information of velocity provided, among others, a fast autonomous landing can be carried out.

The landing maneuver on top of a MP was validated in a $2 \mathrm{D}$ periodic and rectilinear trajectory scenario and a 2D non-periodic and rectilinear trajectory scenario (a vided ${ }^{3}$ of the experiments has been provided as supplementary material). In Fig. 4.8, two experiments were performed for the 2D periodic and rectilinear trajectory scenario, for simulated (Fig. 4.8a) and real-flight (Fig. 4.8b environments. Fig. 4.8a illustrates the variation in velocity of the MP and its estimation $v_{M P}^{W}$ by the UAV. The velocity is averaged in the period of estimation, which is the time the moving platform is underneath the UAV. The period of estimation is highlighted with two dotted lines and is represented by $\Delta t$. Both the 1D-LIDAR measurements and the absolute estimated distance from the ground are also represented. As shown in Fig. 4.8a, two laps of the MP were enough to estimate the velocity, which enables the Fast Landing stage. The third lap triggers the landing stage with the precomputed $t_{\text {land }}$, which is the minimum time to safely land, accounted from the

$\sqrt[3]{\text { https://vimeo.com/205757662 }}$ 
first instant of detection of the platform. The final value is $v_{M P}^{W}=0.66 \mathrm{~m} / \mathrm{s} . t_{\text {land }}=58.37$ $\mathrm{ms}$ is the instant of time when landing is triggered.

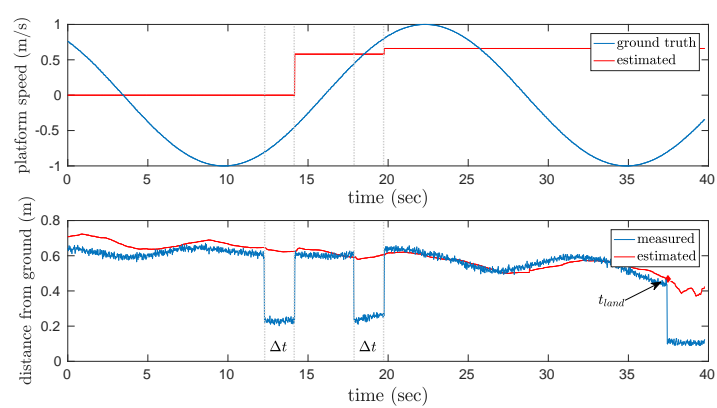

(a)

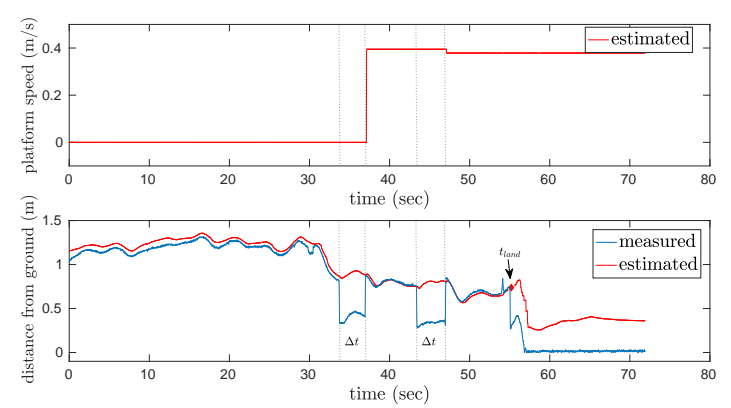

(b)

Figure 4.8: (a) Experiment conducted during the execution of a simulated flight with rectilinear and periodic trajectory of the MP. (Top) Ground truth and estimated velocity of the MP. The final value is $v_{M P}^{W}=0.66 \mathrm{~m} / \mathrm{s}$. (Bottom) Estimated absolute distance from the ground and 1D-LIDAR altimeter measurements. $t_{\text {land }}=58.37 \mathrm{~ms}$ is the instant of time when landing is triggered. (b) Experiment conducted during the execution of a real flight with rectilinear and periodic trajectory of the MP. (Top) Estimated velocity of the MP. The final value is $v_{M P}^{W}=0.3788 \mathrm{~m} / \mathrm{s}$. (Bottom) Estimated absolute distance from the ground and 1D-LIDAR altimeter measurements. $t_{\text {land }}=153.68 \mathrm{~ms}$ is the instant of time when landing is triggered.

The previous experiment was replicated in a real environment, as depicted in Fig. 4.8b, In this scenario, the ground-truth velocity of the platform was not available. Again, two laps were required for a successful landing stage. The final value is $v_{M P}^{W}=0.3788 \mathrm{~m} / \mathrm{s} . t_{\text {land }}=$ $153.68 \mathrm{~ms}$ is the instant of time when landing is triggered. This exact approach was utilized in the 2016 International Micro Aerial Vehicles (IMAV) competition to successfully perform the landing maneuver on top of a MP. The team, which the author of the present thesis by compendium was part of, achieved $4^{\text {th }}$ position in the indoor competition ${ }^{4}$ Additional information about the competition has been provided in Appendix A.4.

In Fig. 4.9, one experiment was performed for the 2D non-periodic and rectilinear trajectory scenario, for a simulated environment. The stated experiment corresponds to the complete solution (which is composed of all the components presented in Fig. 4.7) and the experiment has only been conducted in simulation. For the sake of simplicity, this experiment consisted of a MP describing a non-periodic rectilinear trajectory at a constant velocity of $0.27 \mathrm{~m} / \mathrm{s}$. In a preliminary moment, the UAV estimates the velocity vector of the MP and predicts its future position based on a constant-velocity model. The velocity is estimated at a rate of $100 \mathrm{~Hz}$ for $\Delta t=4.76 \mathrm{~s}$ seconds at $z_{\min }^{W} \simeq 3 \mathrm{~m}$ height (until the algorithm converges). The converged value $\left(v_{M P}^{W}=0.278 \mathrm{~m} / \mathrm{s}\right)$ is taken to enable the fast landing stage. After the estimation phase $\Delta t$, the UAV predicts a position at $d_{\min }^{U} \simeq 8 \mathrm{~m}$ in the future and flies to it $\left(v_{z_{-} \max }^{W} \simeq 0.35 \mathrm{~m} / \mathrm{s}, v_{x y_{-} \max }^{W} \simeq 1.2 \mathrm{~m} / \mathrm{s}\right.$ and $\left.z_{\min }^{M P} \simeq 0.5 \mathrm{~m}\right)$. The performed experiment is enough to validate the stages of estimation, prediction and fast landing for non-periodic trajectories.

These experiments validate the baseline approach for autonomous landing on a MP in certain scenarios. The stated strategy led practical results and served as a first ap-

\footnotetext{
${ }^{4}$ http: //www .imavs .org/2016/documents/Result.pdf
} 


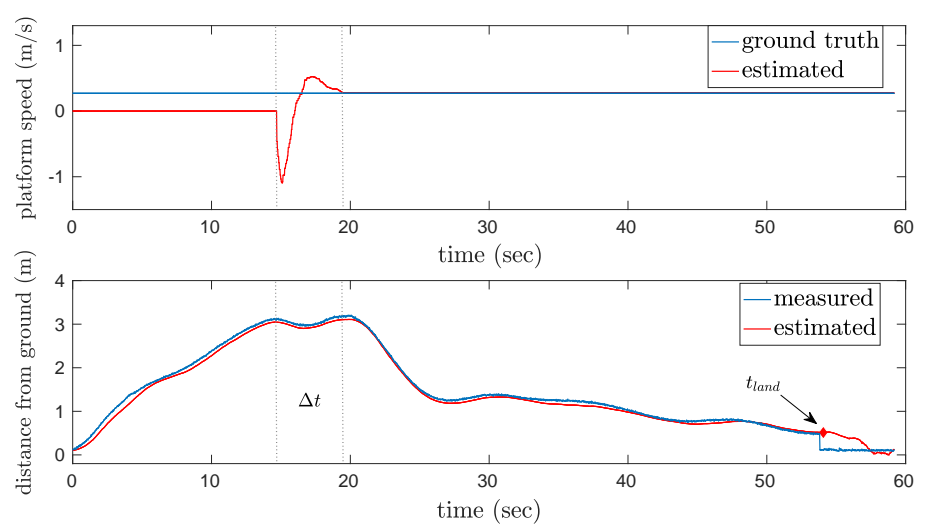

Figure 4.9: Experiment conducted during the execution of a simulated flight with nonperiodic trajectory of the MP. (Top) Ground truth and estimated velocity of the MP. The converged value $\left(v_{M P}^{W}=0.278 \mathrm{~m} / \mathrm{s}\right)$ is taken to enable the fast landing stage. (Bottom) Estimated absolute distance from the ground and 1D-LIDAR measurements. $t_{\text {land }}=269.75$ $\mathrm{ms}$ is the instant of time when landing is triggered.

proximation to the application, under constrained scenarios, such as periodic movement or rectilinear trajectories. However, in P.I, the full problem is tackled, with periodic and non-periodic trajectories, as well as with an arbitrary trajectory setting of the MP.

On the other hand, in Rodriguez-Ramos et al. (2018), the MP was able to move in any arbitrary trajectory, and Position-Based Visual Servoing (PBVS) technique was utilized to perform the autonomous landing maneuver. The MP was assumed to be initiated within the field of view of the multirotor. The position of the MP center in the horizontal axes ( $\mathrm{x}-\mathrm{y}$ plane) was constantly being followed by the multirotor and the altitude was gradually decreased in order to properly land on top of the MP (the thrust of the rotor was automatically cut off when the multirotor was within a safe volume concerning the center of the MP). The complete strategy was based on the RGB camera and IMU from a consumer multirotor, and the position of the MP was estimated based on fiducial markers located on the surface of the MP. The classical PBVS control law is shown in 4.10).

$$
\begin{gathered}
v_{c}=-\lambda \widehat{L_{e}^{-1} \mathrm{e}} \\
{[\theta, \phi, \dot{z}]=k_{1}\left(k_{2} t_{c}[t]+k_{3}\left(t_{c}[t]-t_{c}[t-1]\right)\right)}
\end{gathered}
$$

Where $v_{c}$ represents the velocity reference vector (linear and angular) generated by the PBVS control law in camera frame $(\mathrm{C}), \lambda$ is an adjustable control gain, $L_{e}$ is the interaction matrix and $e$ is the error in translation and rotation with respect to the desired object position, in the camera frame. A conversion between the velocity references generated by the PBVS control law to attitude references has been computed. $t_{c}$ represents the linear velocity component of $v_{c} ; k_{1}, k_{2}$ and $k_{3}$ have been experimentally tuned and have values of $1.45,0.05$, and 2.6 , respectively. $\dot{\psi}$ has been directly forwarded from the yaw component of $v_{c}$ to the hardware interface. The desired altitude has been constantly reduced in every time step to simulate a full landing maneuver.

Several experiments have been carried out in both simulated and real environments for the PBVS classical approach. Each experiment definition is based on the shape and velocity of the MP trajectory (Slow, Fast, 8-Shape, and Runaway). The Slow scenario 
corresponds to a rectilinear periodic trajectory of the MP at a maximum velocity of 0.4 $\mathrm{m} / \mathrm{s}$. Fast scenario corresponds to a rectilinear periodic trajectory of the MP at a maximum velocity of $1.2 \mathrm{~m} / \mathrm{s}$. 8-Shape scenario corresponds to an 8-Shape periodic trajectory of the $\mathrm{MP}$ at a maximum linear velocity of $0.628 \mathrm{~m} / \mathrm{s}$ and with a constant angular velocity of $0.1 \mathrm{rad} / \mathrm{s}$. Runaway scenario corresponds to a rectilinear non-periodic trajectory of the moving platform at a maximum velocity of $1.5 \mathrm{~m} / \mathrm{s}$. In Fig. 4.10, 3D and 2D ground-truth trajectories have been represented for four scenarios in a simulated environment.

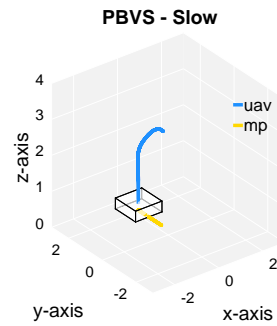

(a)

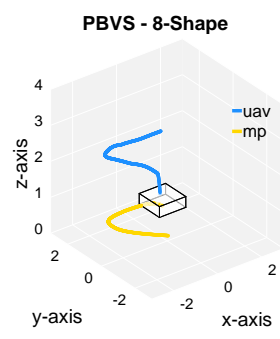

(e)

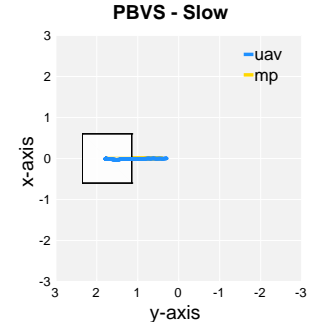

(b)

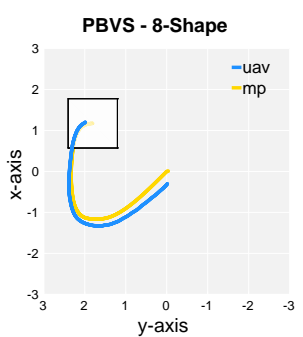

(f)

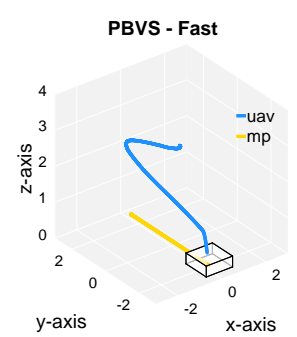

(c)

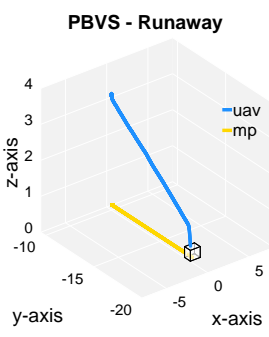

$(\mathrm{g})$

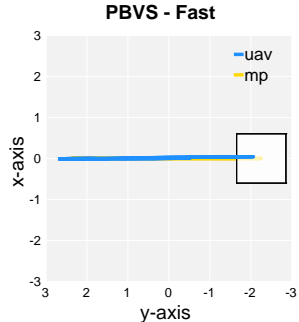

(d)

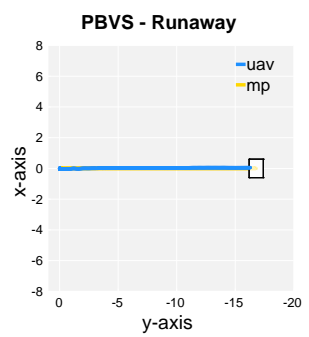

(h)

Figure 4.10: Simulation results corresponding to four different landing scenarios for PBVS approach. (a)(b) Rectilinear periodic trajectory of the MP with a maximum velocity of $0.4 \mathrm{~m} / \mathrm{s}$ (Slow). (c)(d) Rectilinear periodic trajectory of the MP with a maximum velocity of $1.2 \mathrm{~m} / \mathrm{s}$ (Fast). (e)(f) 8-Shape periodic trajectory (with constant angular velocity of $0.1 \mathrm{rad} / \mathrm{s}$ ) of the MP with a maximum velocity of $0.628 \mathrm{~m} / \mathrm{s}$ (8-Shape). (g)(h) Rectilinear non-periodic trajectory of the MP with a maximum velocity of $1.5 \mathrm{~m} / \mathrm{s}$ (Runaway).

In order to validate the PBVS approach in real flights, two different experiments have been performed. As shown in Fig. 4.11, the 3D and 2D ground-truth trajectories of both the multirotor and the MP has been represented for the Fast scenario. For the Runaway scenario, only relative position information has been represented due to the absence of absolute positioning systems. The Fast experiments correspond to a rectilinear periodic trajectory of the MP at a maximum velocity of $0.8 \mathrm{~m} / \mathrm{s}$. The Runaway experiments correspond to a rectilinear non-periodic trajectory of the moving platform at a maximum velocity of $0.86 \mathrm{~m} / \mathrm{s}$.

These experiments, corresponding to the PBVS approach, take a step forward on the performance of the classical method. As shown, they were able to provide a landing maneuver up to $0.86 \mathrm{~m} / \mathrm{s}$ in a real-flight scenario. The approach was not constrained to a specific type of trajectory, being able to be deployed in any arbitrary trajectory scenario, solely utilizing on-board sensors from a consumer multirotor. In P.I, the trajectory was neither constrained to a subset of shapes, being able to be validated in any arbitrary trajectory. Also, the P.I strategy was not based on vision techniques, while in the stated 


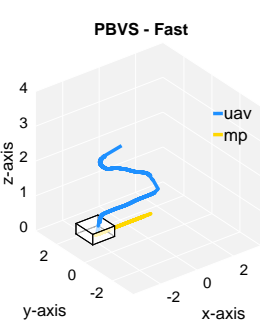

(a)

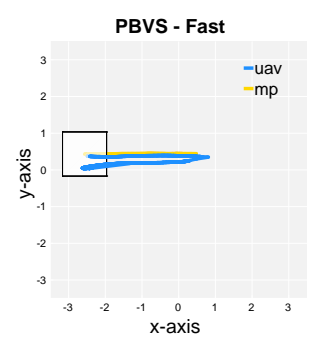

(b)

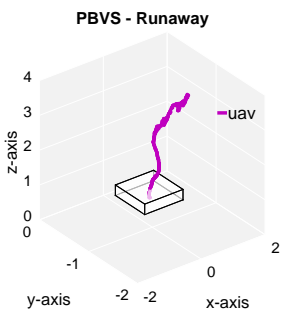

(c)

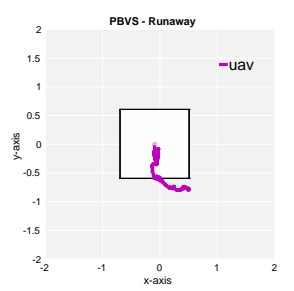

(d)

Figure 4.11: Real-flight results corresponding to two different landing scenarios. (a)(b) Rectilinear periodic trajectory of the MP with a maximum velocity of $0.8 \mathrm{~m} / \mathrm{s}$ (Fast). (c) (d) Rectilinear non-periodic trajectory of the MP with a maximum velocity of $0.86 \mathrm{~m} / \mathrm{s}$. Ground truth data was not available in this experiment. Instead, relative position with respect to the MP has been represented (Runaway).

PBVS approach an RGB camera was the primary sensor. Nevertheless, P.I served as the baseline DRL approach, in order to lay the foundations of an additional vision-based method (explained in Section 4.3.3 which outperformed the stated PBVS technique.

\subsubsection{Reinforcement Learning Based Methods}

The exploration and research of ML-based methods, and in particular, DRL methods in the context of aerial robotics is one of the main goals of this thesis by compendium. Concretely, publication P.I contributed to the establishment of a reinforcement learning framework (see Section 4.2.4 and to the utilization of DRL techniques for multirotor motion control. In P.I the task of landing on top of a MP has been approached with DRL techniques, tackling the full problem with continuous states and action spaces. Additionally, based on publication P.I, in Rodriguez-Ramos et al. (2018), the problem formulation was extended and the approach used vision as its primary information source.

On the one hand, in P.I, the application of autonomous landing on top of a MP was simplified to a $2 \mathrm{D}$ problem to be solved with DRL techniques. DDPG algorithm was utilized to learn the landing maneuver in the 2D space ( $\mathrm{x}-\mathrm{y}$ axes, parallel to the MP plane). The multirotor altitude was constantly decreased in order to finally perform the landing maneuver. This initial formulation allowed for the validation of the stated novel technique and laid the foundations for later studies on a similar subject. The complete policy was learned in a Gazebo simulated environment and with a periodic rectilinear trajectory of the MP. Although the training stage was performed with a periodic and rectilinear trajectory of the MP, the policy was able to generalize enough to any other arbitrary trajectory at testing time. The system architecture of the approach is illustrated in Fig. 4.12 ,

As shown in Fig. 4.12, the agent state was based on the relative position and velocity of the multirotor with respect to the MP, as well as on a contact sensor located on top of the MP, which signalized whether the multirotor touched the MP. The actions provided by the agent, as explained in Section 4.1, corresponded to velocity references (input to a velocity controller) in the $\mathrm{x}-\mathrm{y}$ plane and a constant negative velocity reference in the $\mathrm{z}$-axis.

The approach has been thoroughly validated through several experiments in simulated and real flight scenarios (a vided ${ }^{5}$ has been provided as supplementary material). The full landing maneuver has been trained in simulation throughout 4500 episodes in simulation

\footnotetext{
${ }^{5}$ https://vimeo.com/235350807
} 


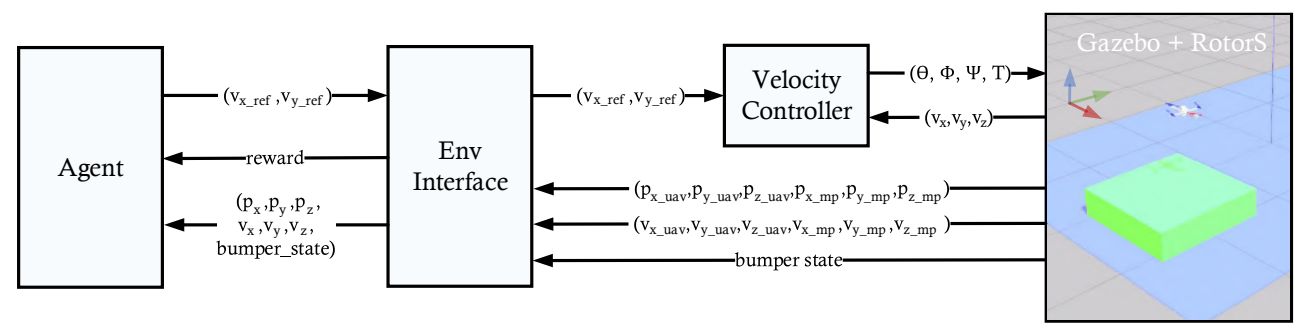

Figure 4.12: Architecture of the proposed P.I reinforcement learning architecture for the case of the experiment of study.

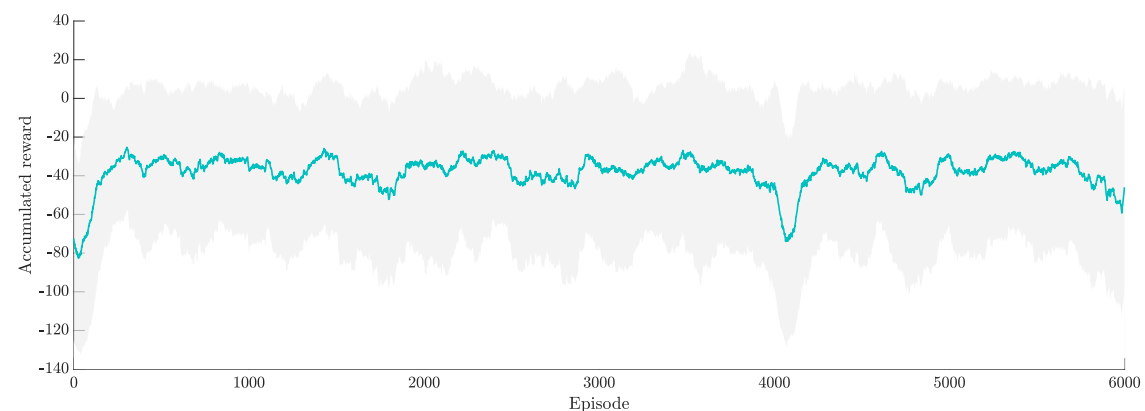

(a)

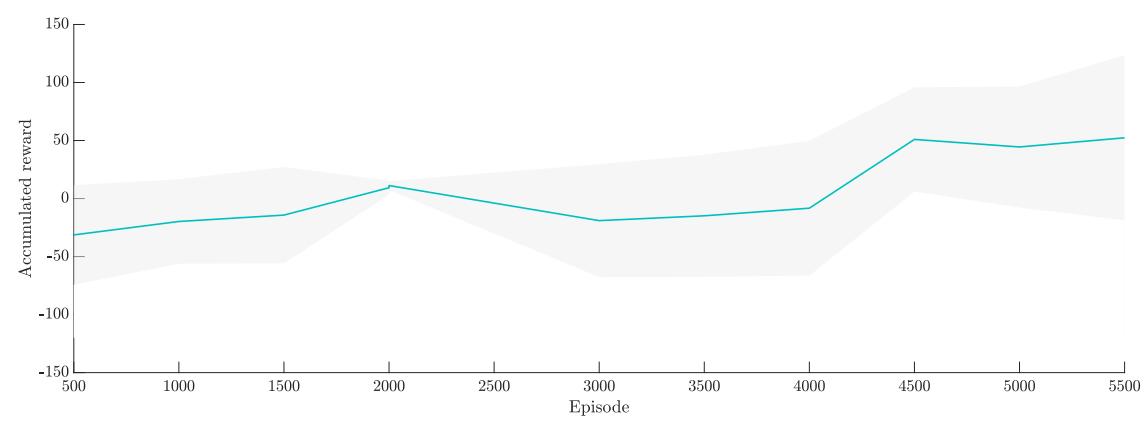

(b)

Figure 4.13: Average and standard deviation of the accumulated reward, for both simulated training and testing phases.

(approximately $720 \mathrm{k}$ training steps over $10 \mathrm{~h}$ ). In this setup, an episode consists of a full landing trial on top of the MP and it is composed of a maximum of 900 training steps.

In Fig. 4.13, the accumulated reward for the training and testing stages is illustrated. The accumulated reward at testing time reached its maximum in episode 4500. Consequently, the policy network of episode 4500, have been selected for further experimentation. Additionally, a simulated experiment has been shown in Fig. 4.14. The position and velocity of the multirotor with respect to the MP in $\mathrm{x}$ and $\mathrm{y}$ axes, as well as the velocity references, generated by the policy of episode 4500 has been illustrated. Finally, in Fig. 4.15, the network has been tested in a real environment, for a periodic trajectory of the MP and an arbitrary trajectory of the MP. A motion capture system was utilized to extract the position of both the multirotor and the MP in order to generate the agent state in real-time.

The robustness of DDPG algorithm has been validated in real flights, with differences in 

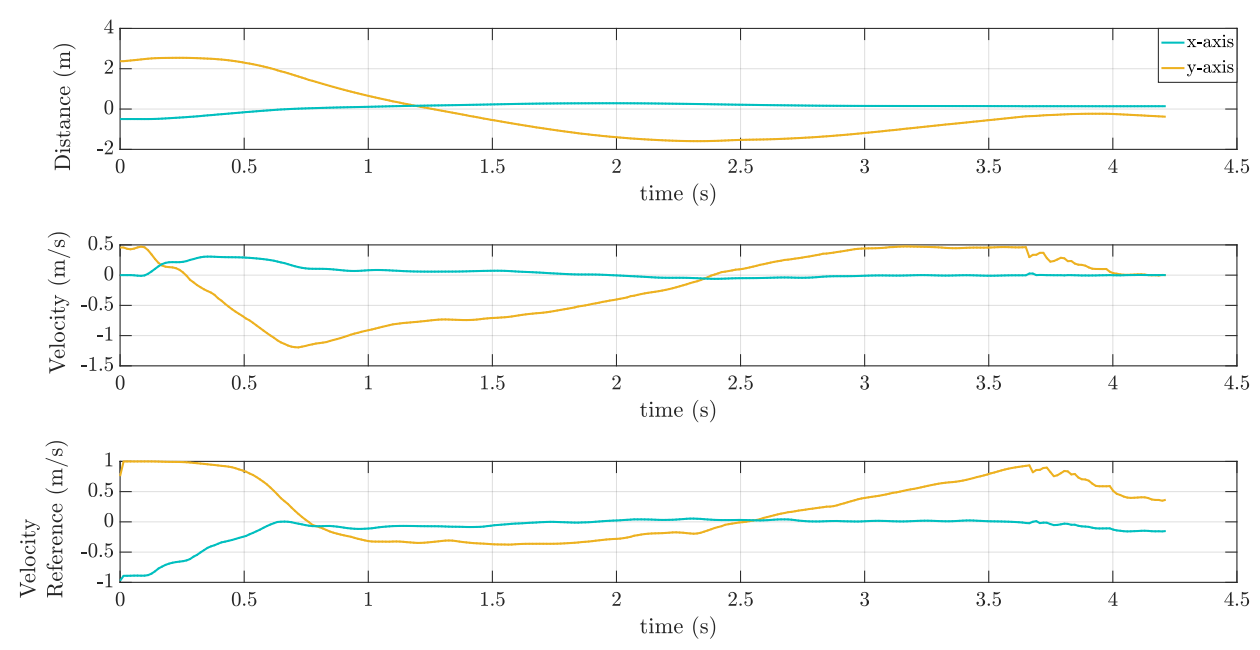

Figure 4.14: Partial state and actions signals of policy network from episode 4500 in simulated environment. (Top) Position of the multirotor with respect to the MP, in $\mathrm{x}$ and $\mathrm{y}$ axes. (Middle) Velocity of the multirotor with respect to the MP, in $\mathrm{x}$ and $\mathrm{y}$ axes. (Bottom) Velocity reference commands (actions) generated by the agent, in world coordinates and in $\mathrm{x}$ and $\mathrm{y}$ axes.

sensors and dynamics of the multirotor used. It has to be noted that no additional tuning of the actions provided by the actor network was required when moving from simulation to real flights. Furthermore, the multirotor included in simulation training and testing phases, and the consumer multirotor used in real-flight testing have a similar size but the simulated dynamics differ from the real ones. The dynamics of the simulated multirotor were not required to be adjusted in simulation to match real multirotor dynamics. In this context, the generalization capability of the DDPG algorithm was enough to overcome stated differences. Also, P.I approach performs high-level control (velocity reference control), so that differences in dynamics can be partially absorbed by proper tuning of the velocity and autopilot controllers.

On the other hand, in Rodriguez-Ramos et al. (2018), an extension of P.I has been carried out. The problem formulation has been extended, in order to include the 3-DOF of the landing maneuver, to provide the agent the ability to cut off the thrust at any moment and to complete the approach with vision-based techniques. The primary sensors for state estimation were an RGB camera and the IMU from a consumer multirotor. In this approach, fiducial markers (ArUco markers Garrido-Jurado et al. (2016)) have been utilized to generate the position of the multirotor with respect to the MP. In Fig. 4.19 the architecture of the Rodriguez-Ramos et al. (2018) approach has been illustrated. On this subject, the framework partially developed in P.I (see Section 4.2.4 was extended to enable the vision-based landing application. The DDPG agent has been trained over 10,000 episodes in simulation (approximately over $10 \mathrm{~h}$ ). The network of episode 4800 has provided the best results in terms of landing performance for different scenarios and has been selected for further experimentation. The architecture was versatile enough to be easily adaptable from simulated to real flights.

Several experiments have been conducted in both simulated and real-flight environments (a vided ${ }^{6}$ has been provided as supplementary material and the code has been made

\footnotetext{
https://vimeo.com/259315997
} 

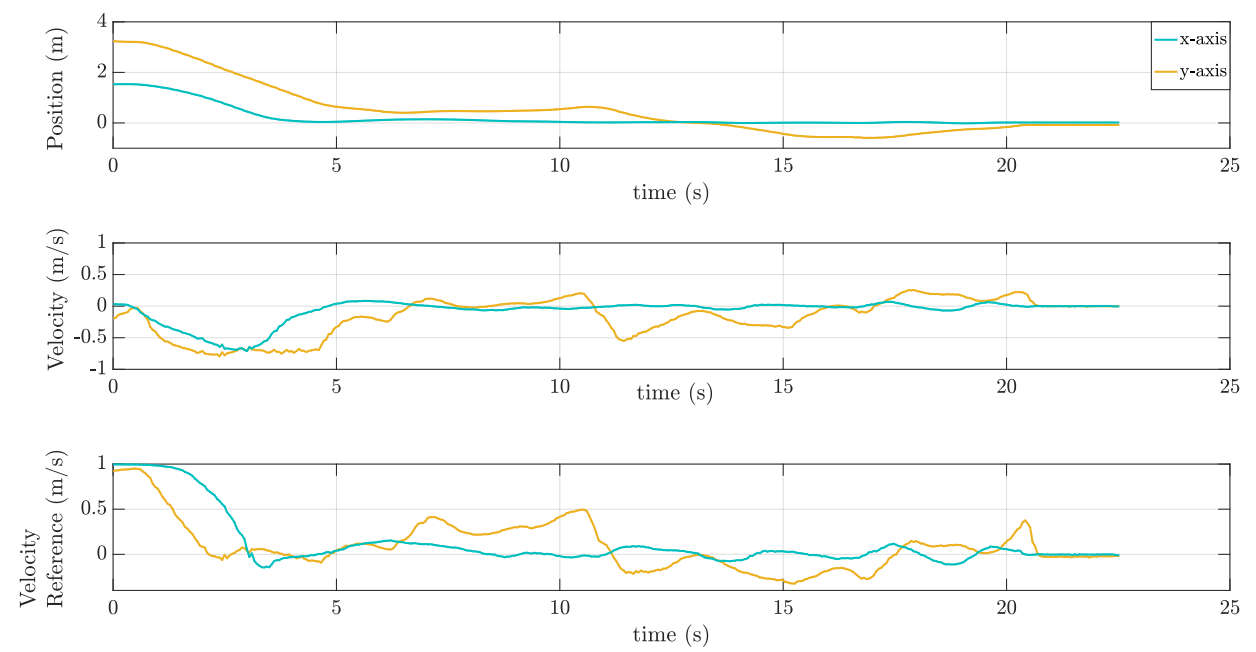

(a)
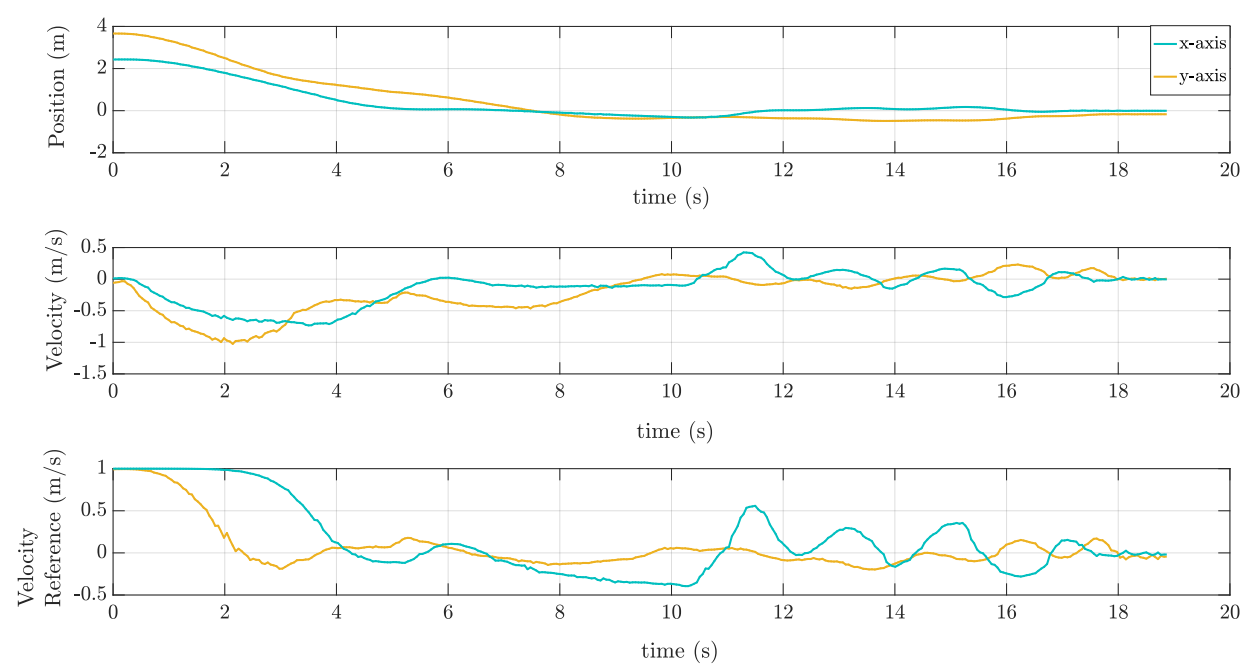

(b)

Figure 4.15: Partial state and actions signals of policy network from episode 4500 in a realflight environment, for (a) a periodic trajectory of the MP and (b) an arbitrary trajectory of the MP. (Top) Position of the multirotor with respect to the MP, in $\mathrm{x}$ and $\mathrm{y}$ axes. (Middle) Velocity of the multirotor with respect to the MP, in $\mathrm{x}$ and $\mathrm{y}$ axes. (Bottom) Velocity reference commands (actions) generated by the agent, in world coordinates and in $\mathrm{x}$ and $\mathrm{y}$ axes.

publicly available ${ }^{7}$. Similarly to the PBVS approach in Section 4.3.2, each experiment definition is based on the shape and velocity of the MP trajectory (Slow, Fast, 8-Shape, and Runaway). The Slow scenario corresponds to a rectilinear periodic trajectory of the $\mathrm{MP}$ at a maximum velocity of $0.4 \mathrm{~m} / \mathrm{s}$. Fast scenario corresponds to a rectilinear periodic trajectory of the MP at a maximum velocity of $1.2 \mathrm{~m} / \mathrm{s}$. 8-Shape scenario corresponds to an 8-Shape periodic trajectory of the MP at a maximum linear velocity of $0.628 \mathrm{~m} / \mathrm{s}$ and with a constant angular velocity of $0.1 \mathrm{rad} / \mathrm{s}$. Runaway scenario corresponds to a rectilin-

7https://github.com/alejodosr/drl-landing 


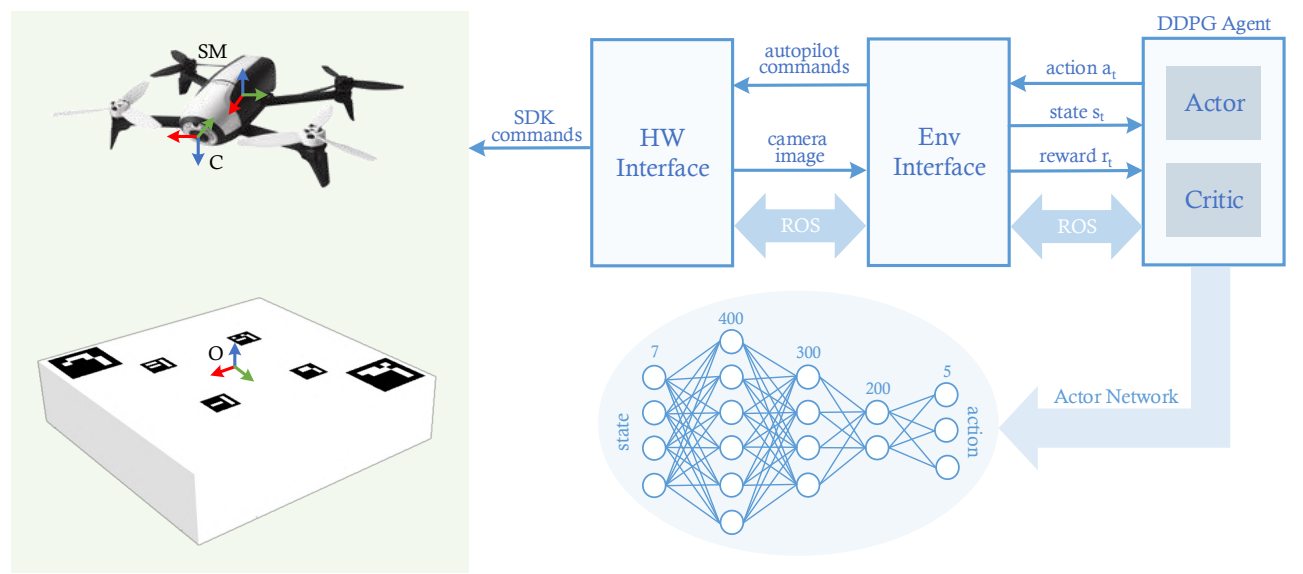

Figure 4.16: Architecture of the proposed DRL based landing system in Rodriguez-Ramos et al. (2018). All the frames of reference involved and the networks structure are depicted.

ear non-periodic trajectory of the moving platform at a maximum velocity of $2 \mathrm{~m} / \mathrm{s}$. In Fig. 4.17, 3D and 2D ground-truth trajectories have been represented for four scenarios in a simulated environment.

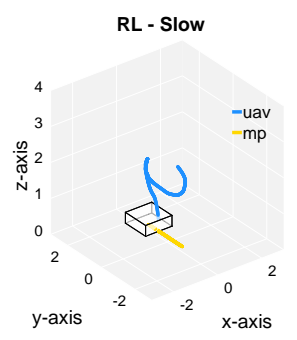

(a)

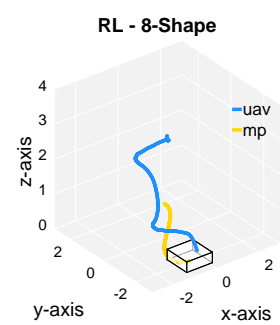

(e)

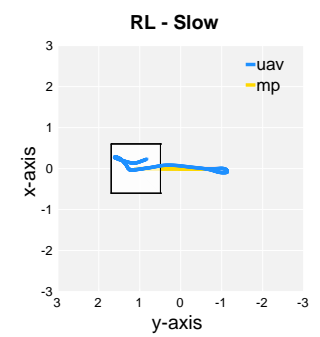

(b)

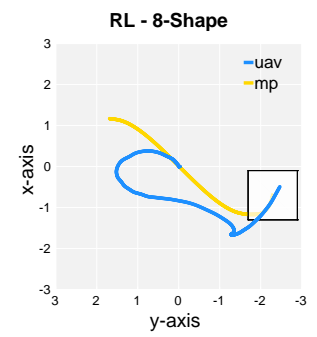

(f)

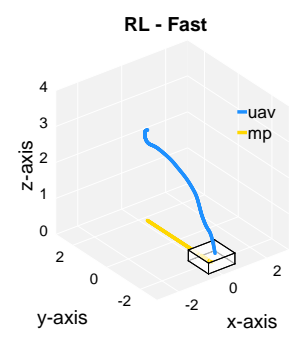

(c)

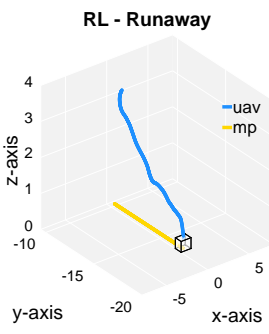

$(\mathrm{g})$

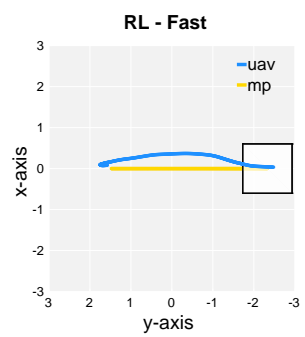

(d)

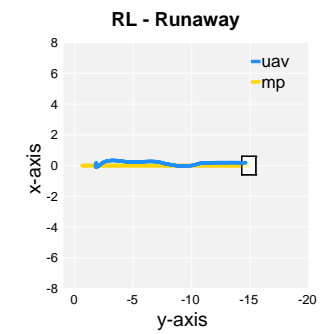

(h)

Figure 4.17: Simulation results corresponding to four different landing scenarios for DRL approach. (a)(b) Rectilinear periodic trajectory of the MP with a maximum velocity of $0.4 \mathrm{~m} / \mathrm{s}$ (Slow). (c)(d) Rectilinear periodic trajectory of the MP with a maximum velocity of $1.2 \mathrm{~m} / \mathrm{s}$ (Fast). (e)(f) 8-Shape periodic trajectory (with constant angular velocity of $0.1 \mathrm{rad} / \mathrm{s}$ ) of the MP with a maximum velocity of $0.628 \mathrm{~m} / \mathrm{s}$ (8-Shape). (g) (h) Rectilinear non-periodic trajectory of the MP with a maximum velocity of $2 \mathrm{~m} / \mathrm{s}$ (Runaway).

In order to validate the DRL approach in real flights, two different experiments have been performed. As shown in Fig. 4.18, the 3D, and $2 \mathrm{D}$ ground-truth trajectories of both 
the multirotor and the MP has been represented for the Fast scenario. For the Runaway scenario, only relative position information has been represented due to the absence of absolute positioning systems. The Fast experiments correspond to a rectilinear periodic trajectory of the MP at a maximum velocity of $0.8 \mathrm{~m} / \mathrm{s}$. The Runaway experiments correspond to a rectilinear non-periodic trajectory of the moving platform at a maximum velocity of $0.86 \mathrm{~m} / \mathrm{s}$.

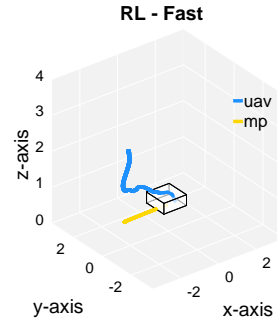

(a)

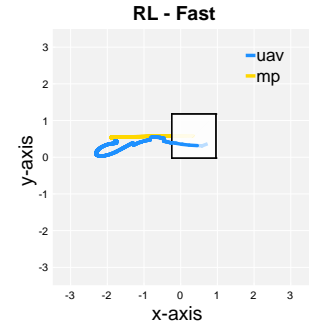

(b)

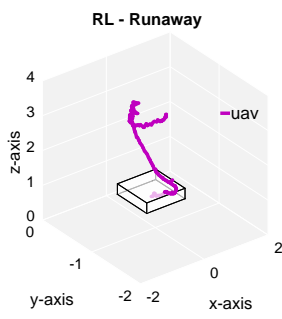

(c)

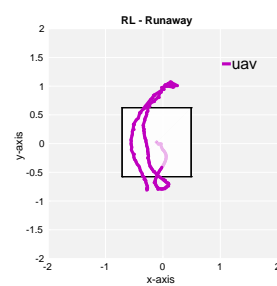

(d)

Figure 4.18: Real-flight results corresponding to two different landing scenarios. (a)(b) Rectilinear periodic trajectory of the MP with a maximum velocity of $0.8 \mathrm{~m} / \mathrm{s}$ (Fast). (c) (d) Rectilinear non-periodic trajectory of the MP with a maximum velocity of $0.86 \mathrm{~m} / \mathrm{s}$. Ground truth data was not available in this experiment. Instead, relative position with respect to the MP has been represented (Runaway).

The extensive validation of the approaches proposed in P.I and Rodriguez-Ramos et al. (2018) reveals the proper behavior of the agents in both simulated and real-flight scenarios. The agents, which have been only trained in simulation with one simple scenario (the periodic and rectilinear trajectory of the MP) were able to generalize enough to perform in other different scenarios and in real-flight experiments. A powerful approach has been validated: it is possible to train complex behaviors in simulation and use them in the real world. Indeed, similarities between the simulated and the real environment are necessary. However, for instance, the multirotor dynamics has not been required to be modeled for simulated environments, since the networks have been able to overcome these differences and the velocity controllers or autopilots (P.I and Rodriguez-Ramos et al. (2018), respectively) were properly tuned to abstract the networks from stated differences in dynamics.

Additional data efficiency is required for DRL algorithms (10 hours over 5500 episodes and 10 hours over 5000 episodes have been necessary to train our selected agents, for P.I and Rodriguez-Ramos et al. (2018), respectively). Nevertheless, the performance notably improves after a few hours of training. The DRL landing approach Rodriguez-Ramos et al. (2018) is able to perform more aggressive maneuvers. In the Runaway scenario, the DRL landing approach is able to land at $2 \mathrm{~m} / \mathrm{s}$ while PBVS landing approach (see Section 4.3 .2 lands at a maximum velocity of $1.5 \mathrm{~m} / \mathrm{s}$.

In real flights (see Fig. 4.15 and Fig. 4.18, both DRL algorithms show practical performances, being able to land on top of the MP in a wide variety of conditions. In P.I additional sensory infrastructure (i.e. motion capture system) was necessary to perform the landing maneuver. In Rodriguez-Ramos et al. (2018), a pure vision-based approach was carried out without additional sensory infrastructure. The computation overhead of both approaches was reduced, being able to be launched on an average CPU and with a consumer multirotor. 


\subsection{Autonomous Multirotor Following (Publication P.II)}

In publication P.II, the autonomous multirotor following has been approached from a DL and DRL perspective using synthetic data techniques. This section explains the most relevant aspects of the P.II approach and its outcomes. Also, this section is in accordance with C.2 - C.4 and fulfills objectives 0.1 - O.6.

\subsubsection{Introduction}

Learning techniques aided by synthetic information are increasingly popular in the robotics field, mostly due to the lack of available real-domain data information for applicationspecific tasks, e.g. low-level motion control for complex robot dynamics Tan et al. (2018); Rodriguez-Ramos et al. (2018); Hwangbo et al. (2017) or vision-based strategies for unusual scenarios (corner cases) Rozantsev et al. (2015b); Tobin et al. (2017); Chen et al. (2018).

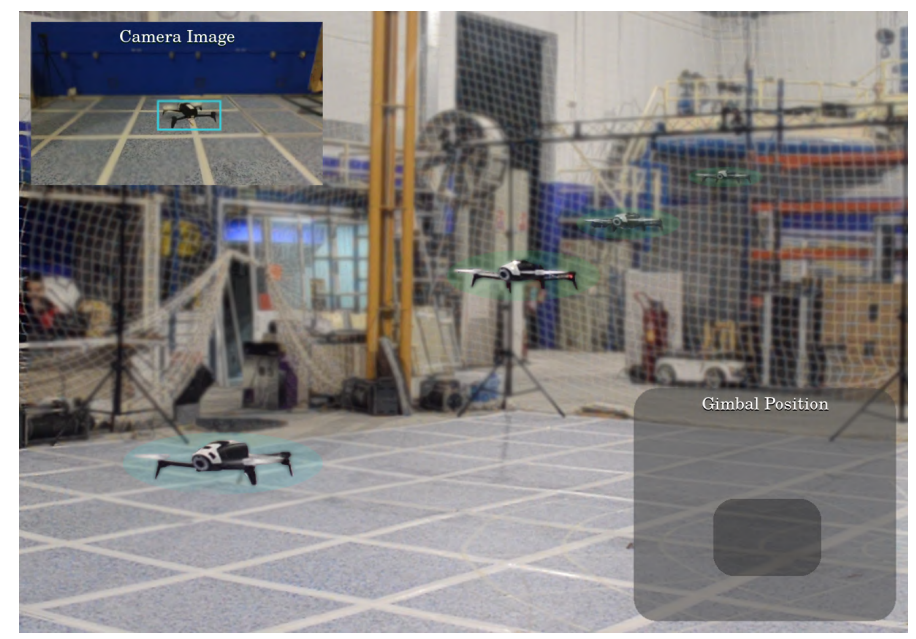

Figure 4.19: Image composition of a non-cooperative multirotor (light-blue) followed by another multirotor that implemented the P.II synthetic-learning based approach (lightgreen) in the real experimentation environment.

Furthermore, in the context of DL, the amount of data required for the stability of learning and model generalization has grown several orders of magnitude, which makes dataset generation harder. State-of-the-art DRL techniques are also still notably sampleinefficient Mnih et al. (2015), which leads to needing a high number of agent-environment interactions (see Section 4.3.3p. This extensive amount of environment interactions is normally not possible in the real world due to system stability, representative sample gathering, or power availability. On this basis, in order to achieve scalability and efficiency of training for both DL and DRL techniques, the exploration of synthetic learning strategies in robotics is a matter of importance that is not yet resolved. Additionally, this paradigm is potentially valuable when annotated datasets are not available.

In this scenario, a multirotor can be a suitable robotic test bench to validate this type of synthetic techniques for robotic applications. Their inherent open-loop system instability, which can create a stressful scenario for the technique under research, and their low-cost RGB camera, which can lead to a wide variety of applications, make this platform a suitable target for novel motion-control research, as well as for vision-based strategies.

On the other hand, in P.II, the task of autonomous following of a non-cooperative multirotor has been selected as the application under research. It has been extracted from 
the proposed Challenge 1 (out of three challenges) of the 2020 Mohammed Bin Zayed International Robotics Competition (MBZIRC). Indeed, publication P.II served as a first approximation, from a DL and DRL perspective to the proposed challenge. The team, which the author of the present thesis by compendium was part of as the lead role, ended up in $3^{r d}$ position in the Grand Challeng 8 , which was a parallel combination of the three proposed challenges. Additional information about the competition has been provided in Appendix A

The strategy has been approached with solely passive and proprioceptive sensors, such as an RGB camera and an IMU, respectively. Also, the camera was provided with 2 additional DOF, thanks to an on-board camera gimbal. The most relevant components of the complete technique are a DL-based multirotor detector and a DRL-based motioncontrol policy to perform the multirotor following maneuver. These components are best explained in the following sections.

\subsubsection{Deep Learning Based Methods}

The first component of the P.II approach has been a DL-based multirotor detector. The detector used in this study was a custom implementation of the one-stage RetinaNet detector Lin et al. (2017), which is a single unified network composed of a backbone network and two task-specific subnetworks (see Fig. 4.20). The backbone is responsible for computing a convolutional and pyramidal feature map over an entire input and is an off-the-shelf convolutional network. In this work, ResNet50 He et al. (2016) was selected as the full network backbone in order to find a balanced trade-off between accuracy and image throughput $\mathrm{He}$ et al. (2016).

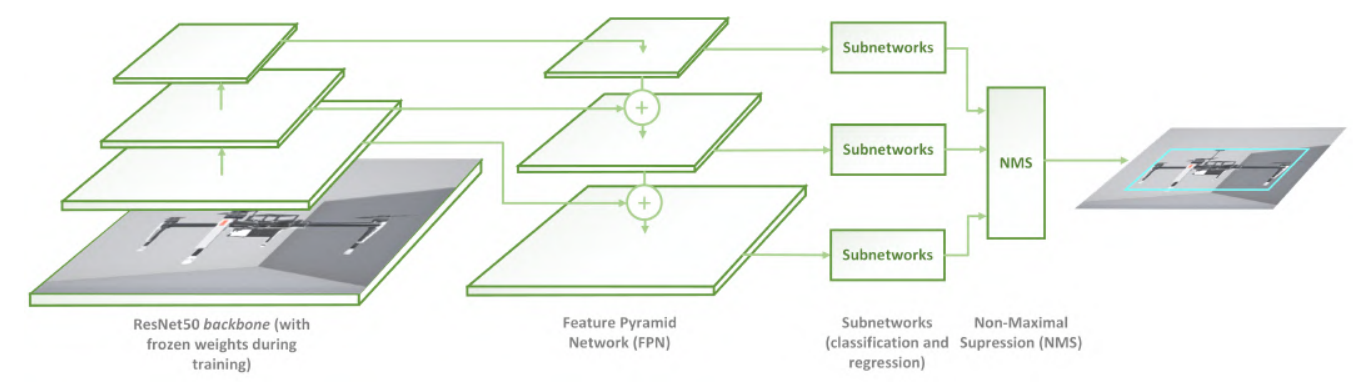

Figure 4.20: Architecture of proposed multirotor detector, based on RetinaNet architecture, with ResNet50 backbone and five levels for Feature Pyramid Network (FPN; fewer levels represented in figure for the sake of simplicity). Backbone weights were frozen during training. Final subnetworks were fine-tuned with pure synthetic multirotor images.

The stated detector has to be trained for the task of multirotor detection. However, in-flight multirotor datasets are a scarce resource, since they are not commonly available to the public community. This context matches one of the key benefits of synthetic data, which is the data generated when the real-domain data is not available. In this regard, multirotor images from a game-engine were synthetically generated. The images were built on different multirotor perspectives and distances with simple grey background. In Fig. 4.21, training examples corresponding to the synthetic dataset are illustrated. The dataset has been made publicly available ${ }^{9}$.

In P.II, for the approach to be successful with the synthetic dataset, the detector

$\sqrt[8]{\text { https://www.mbzirc.com/winning-teams/2020/challenge4 }}$ 


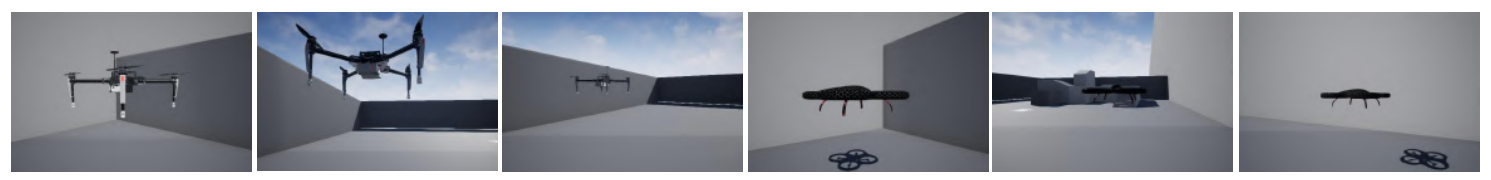

Figure 4.21: Examples of the synthetic images corresponding to the generated dataset.

network was pretrained on generic and extensively-available images from the COCO dataset Lin et al. (2014). As a latter stage, the model was fine-tuned with the synthetic dataset (new synthetic multirotor class not available in COCO dataset). Concretely, the synthetic dataset was mainly composed of two synthetic multirotor models (Parrot ArDrone and DJI Matrice 100), and a small subset of birds and cars extracted from the COCO dataset. It has been found that, with the addition of these two extra classes, the total number of false positives was decreased. A small multirotor-validation dataset composed of real images was also annotated in order to select the most appropriate network and to provide training and validation results. The network was fine-tuned for six epochs $(10,000$ update steps each with frozen backbone weights) and selected the best one in terms of mean Average Precision (AP) performance. The training and validation results are shown in Table 4.1. The AP was computed for a minimum Intersection over Union (IoU) of [0.5:0.05:0.95], 0.5, and 0.75 ( $\mathrm{AP}, \mathrm{AP}_{50}$, and $\mathrm{AP}_{75}$, respectively).

Table 4.1: Average Precision (AP) was computed for a minimum Intersection over Union (IoU) of [0.5:0.05:0.95], 0.5 and 0.75 ( $\mathrm{AP}, \mathrm{AP}_{50}$ and $\mathrm{AP}_{75}$, respectively). The network of epoch 0 corresponds to a special case, where network was not yet trained with synthetic images (only with real images of non-multirotor classes from COCO dataset) and was listed to make more salient the effect of our synthetic dataset. The network of epoch 4 was selected for experimentation due to its capability of generalization.

\begin{tabular}{|c|c|c|c|c|c|c|c|c|c|c|c|c|}
\hline \multirow[b]{2}{*}{ Epoch } & \multicolumn{3}{|c|}{$\begin{array}{c}\text { Training } \\
\text { (only NC-M) }\end{array}$} & \multicolumn{3}{|c|}{$\begin{array}{c}\text { Validation } \\
\text { (only NC-M) }\end{array}$} & \multicolumn{3}{|c|}{$\begin{array}{l}\text { Training } \\
\text { (full) }\end{array}$} & \multicolumn{3}{|c|}{$\begin{array}{l}\text { Validation } \\
\text { (full) }\end{array}$} \\
\hline & $\mathrm{AP}$ & $\mathrm{AP}_{50}$ & $\mathrm{AP}_{75}$ & $\mathrm{AP}$ & $\mathrm{AP}_{50}$ & $\mathrm{AP}_{75}$ & $\mathrm{AP}$ & $\mathrm{AP}_{50}$ & $\mathrm{AP}_{75}$ & $\mathrm{AP}$ & $\mathrm{AP}_{50}$ & $\mathrm{AP}_{75}$ \\
\hline $0^{*}$ & 0.73 & 0.98 & 0.54 & 0.24 & 0.35 & 0.30 & 0.30 & 0.58 & 0.54 & 0.59 & 0.73 & 0.69 \\
\hline 1 & 0.91 & 0.99 & 0.99 & 0.25 & 0.35 & 0.31 & 0.69 & 0.83 & 0.76 & 0.73 & 0.96 & 0.85 \\
\hline 2 & 0.92 & 0.99 & 0.99 & 0.63 & 0.93 & 0.70 & 0.76 & 0.88 & 0.83 & 0.75 & 0.96 & 0.87 \\
\hline 3 & 0.94 & 0.99 & 0.99 & 0.61 & 0.9 & 0.70 & 0.76 & 0.88 & 0.83 & 0.73 & 0.95 & 0.85 \\
\hline 4 & 0.93 & 0.99 & 0.99 & 0.64 & 0.93 & 0.75 & 0.85 & 0.95 & 0.92 & 0.75 & 0.97 & 0.89 \\
\hline 5 & 0.95 & 0.99 & 0.99 & 0.61 & 0.89 & 0.69 & 0.95 & 0.99 & 0.98 & 0.74 & 0.95 & 0.87 \\
\hline 6 & 0.95 & 0.99 & 0.99 & 0.60 & 0.89 & 0.68 & 0.95 & 0.98 & 0.98 & 0.73 & 0.96 & 0.88 \\
\hline
\end{tabular}

The network of Epoch 4 provided the highest validation AP and has been used for further experimentation of the complete approach. It sufficiently generalized not only for the multirotor used in P.II work but for a wide variety of multirotors of the validation dataset (see Table 4.1). Epoch 0 of training showed inadequate network performance, while the following epochs showed an abrupt growth of the AP for both partial and full datasets. The partial dataset corresponded to solely one multirotor class and the complete dataset included three classes (multirotor, birds, and cars). In Fig. 4.22, examples of detection on real images of the validation dataset have been depicted. For clarity, both true positives and false negatives have been shown for the multirotor, bird, and car classes. Although there are false negatives on the validation dataset, the achieved AP shows outstanding results for the application under research. These results validated P.II technique and confirmed the benefits of the inclusion of the synthetic dataset. Additionally, this trend has enabled numerous applications based on multirotor detection and drafted a training pipeline for 

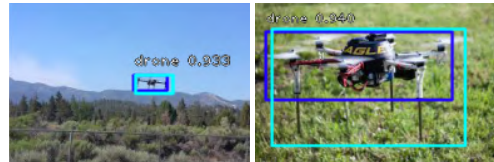

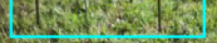
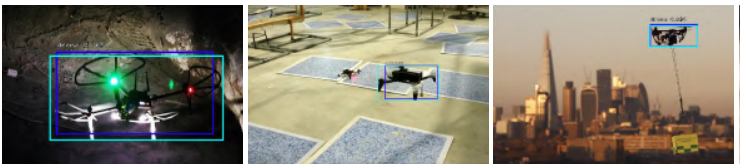

(a)
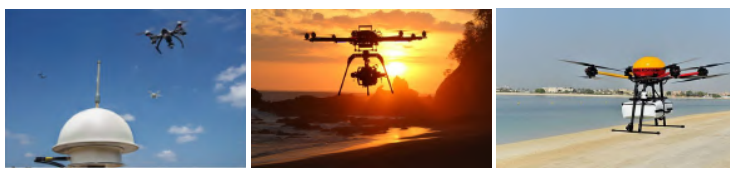

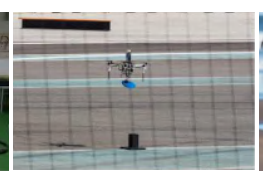

(b)
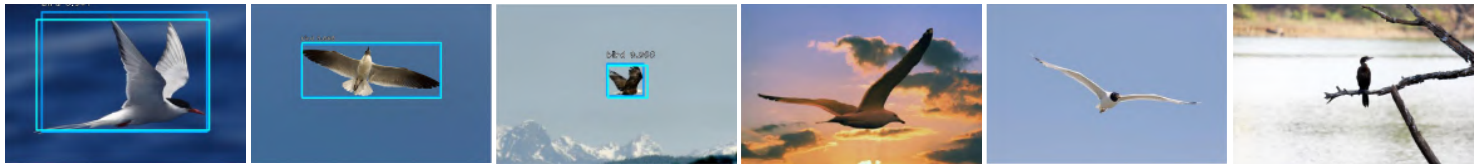

(c)
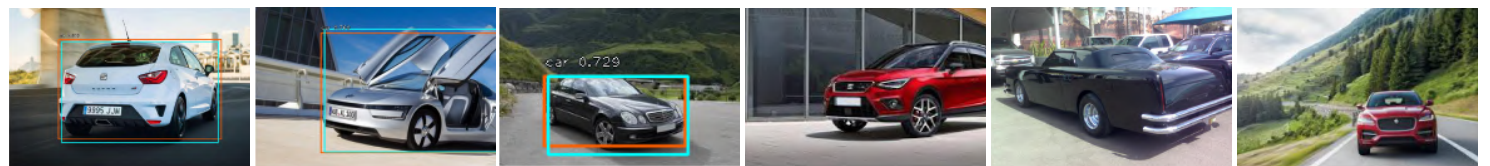

(d)

Figure 4.22: Examples of the real images corresponding to the validation dataset. Both true positives and false negatives have been shown for the multirotor, bird and car classes.

unavailable annotated datasets, such as the case of this study.

\subsubsection{Reinforcement Learning Based Methods}

The second component of the P.II approach has been a DRL-based motion-control policy, in charge of implementing the autonomous multirotor following behavior. Nevertheless, it has to be highlighted that a state-of-the-art object tracker (ECO tracker Danelljan et al. (2017)) has also been included in order to stabilize the RoI predicted by the detector (see Section 4.4.3), as well as to be able to provide a candidate RoI in every frame of the incoming video images. In P.II, the problem formulation was extended, concerning P.I and Rodriguez-Ramos et al. (2018), since the agent state was pure image-based and the continuous action space included 3-DOF of the multirotor and 2-DOF of the camera gimbal. In Fig. 4.23 the architecture of the P.II approach has been illustrated. On this subject, the framework partially developed in P.I (see Section 4.2.4) was extended in P.II to enable the vision-based multirotor following application. The PPO agent has been trained over 4432 update steps in simulation, with trajectories of 256 experiences (approximately over $16 \mathrm{~h}$ ). A DDPG agent has also been trained over $48 \mathrm{~h}$, though it has provided inferior results in terms of maximum accumulated reward reached and global performance for the task under study. PPO actor-critic network of update step 4220 has provided the best results in terms of performance for the scenarios presented in P.II and has been selected for further experimentation. The architecture was versatile enough to be easily adaptable from simulated to real flights.

Several experiments have been conducted in both simulated and real-flight environ- 


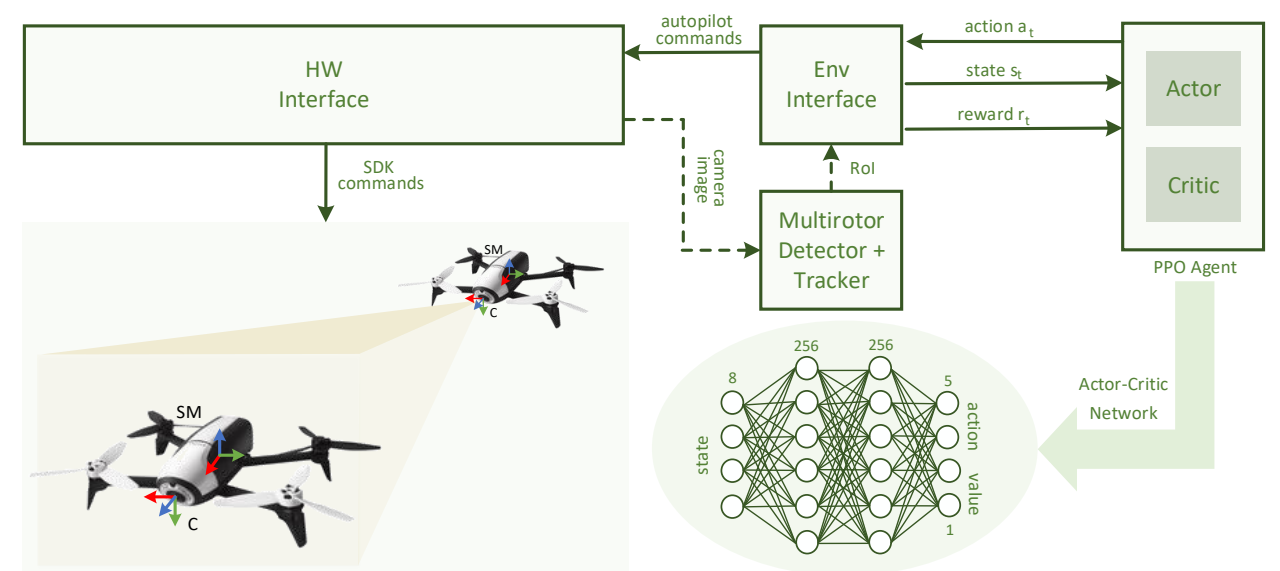

Figure 4.23: Architecture of the proposed DRL-based autonomous multirotor following task, included in P.II. All the frames of reference involved and the networks structure are depicted.

ments (a video ${ }^{10}$ has been provided as supplementary material). Each experiment definition is based on the shape of the Non-Cooperative Multirotor (NC-M) trajectory (X-Axis, $Y$-Axis, Z-Axis, and Arbitrary). The $X$-Axis corresponds to a periodic and rectilinear trajectory along the x-axis. $Y$-Axis corresponds to a periodic and rectilinear trajectory along the y-axis. Z-Axis corresponds to a periodic and rectilinear trajectory along the z-axis. Arbitrary corresponds to a non-periodic and arbitrary trajectory. In Fig. 4.24 several simulated experiments in stated scenarios have been illustrated. 3D and 2D trajectories of both the following multirotor and the NC-M has been depicted. Also, the RoI center error with respect to the center of the image and the RoI area error with respect to the target RoI area have been included for clarity. The agent has managed to follow the NC-M at a maximum velocity of $1.3 \mathrm{~m} / \mathrm{s}$ for $X$-Axis and $Y$-Axis in simulation. In Fig. 4.25 several real-flight experiments in stated scenarios have been illustrated. The agent has managed to follow the NC-M at a maximum velocity of $0.3 \mathrm{~m} / \mathrm{s}$ for every scenario in real flights.

The simulated and real-world environments were similar but not perfect, such as the autopilot response or the multirotor dynamics. Indeed, a decrease in performance of the approach in real-flight experiments compared to the simulated scenarios has been found (refer to maximum velocities in Fig. 4.24 and Fig. 4.25). This can be due to differences between real and simulated multirotor dynamics, autopilot response, and the camera gimbal model. Nevertheless, even with a decrease in performance, the transition of the approach from simulation to real-world was direct and the complete approach utilized synthetic information for every component development, which was the overall aim of this work. P.II approach showed outstanding performance in all experiment scenarios. In comparison to P.I, publication P.II provided a complete image-based technique and a high-dimensional continuous-state solution (5-DOF) for the DRL agents. In P.II, the multirotor was able to keep track of the NC-M during the whole set of tests. The processing overhead of both multirotor detector and motion-control policy was reduced and could be executed in an average CPU (motion-control policy) and GPU (multirotor detector).

\footnotetext{
${ }^{10}$ https://vimeo.com/352940150
} 


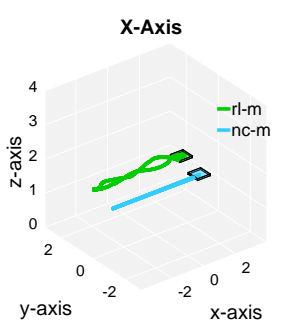

(a)

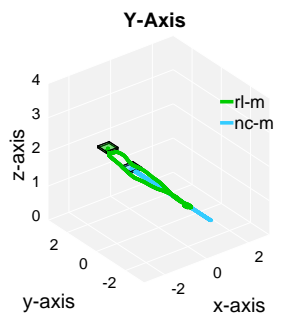

(e)

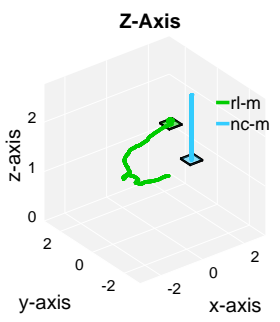

(i)

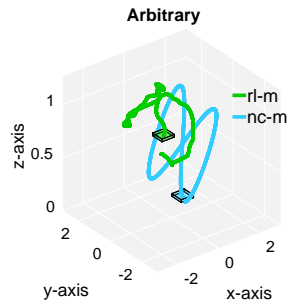

(m)

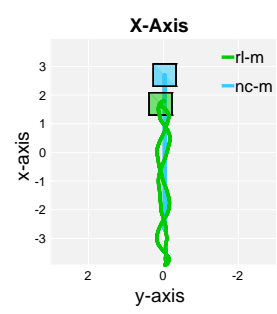

(b)

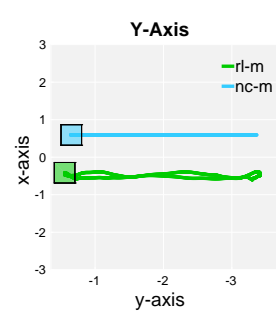

(f)

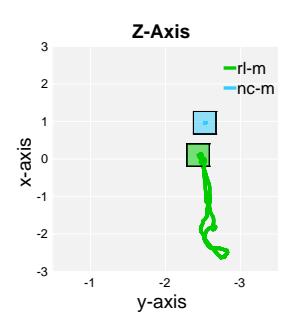

(j)

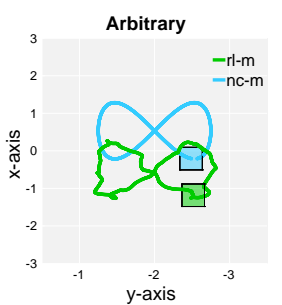

(n)

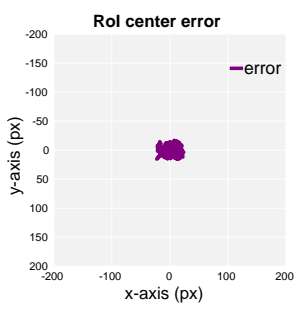

(c)

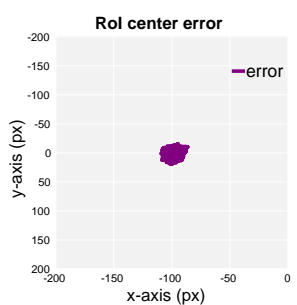

(g)

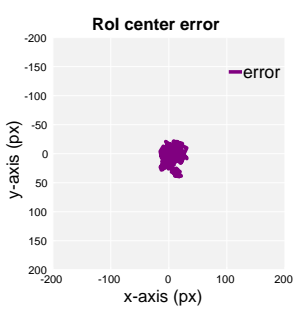

(k)

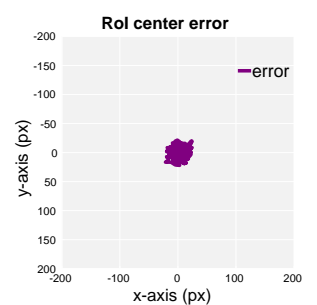

(o)

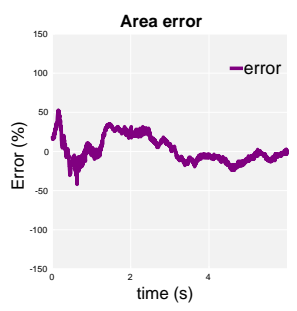

(d)

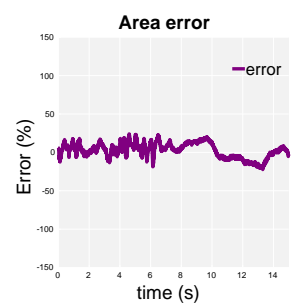

(h)

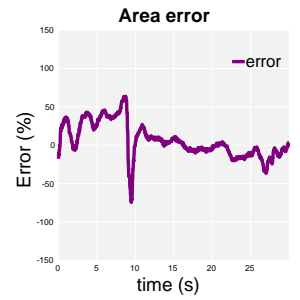

(1)

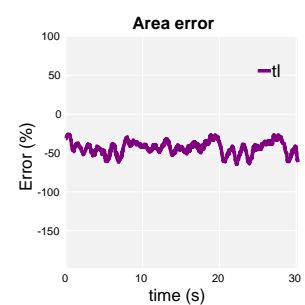

(p)

Figure 4.24: Simulation results corresponding to four different scenarios for the P.II multirotor following approach. (a)(b)(e)(f) Rectilinear periodic trajectory of the NonCooperative Multirotor (NC-M) along the $\mathrm{x}$ and $\mathrm{y}$ axes with a maximum velocity of $1.3 \mathrm{~m} / \mathrm{s}$ (X-Axis and $Y$-Axis, respectively). (i)(j) Rectilinear periodic trajectory of the NC-M along $\mathrm{z}$ axis with a maximum velocity of $0.5 \mathrm{~m} / \mathrm{s}$ (Z-Axis). (m)(n) Arbitrary non-periodic trajectory of the NC-M with a maximum velocity of $0.47 \mathrm{~m} / \mathrm{s}$ (Arbitrary). $(\mathrm{c})(\mathrm{d})(\mathrm{g})(\mathrm{h})(\mathrm{k})(\mathrm{l})(\mathrm{o})(\mathrm{p})$ Current-target RoI center and area error corresponding to each of the four stated experiments. Multirotor trajectories have been generated by a groundtruth source.

\subsection{Adaptive Video Object Detection (Publication P.III)}

In publication P.III, an adaptive video object detection technique has been proposed. This section explains the most relevant aspects of the P.III approach and its outcomes. Also, 


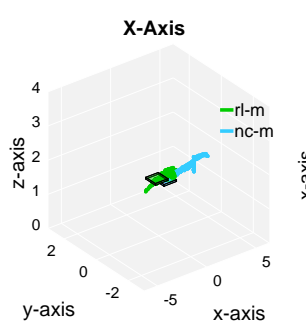

(a)

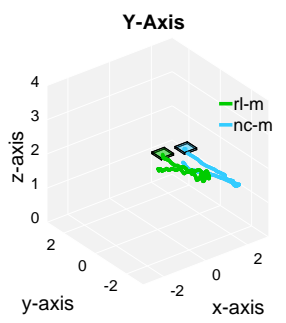

(e)

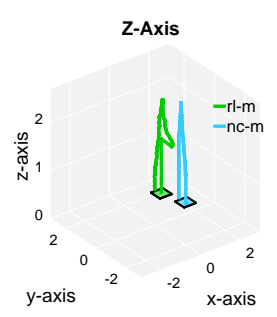

(i)

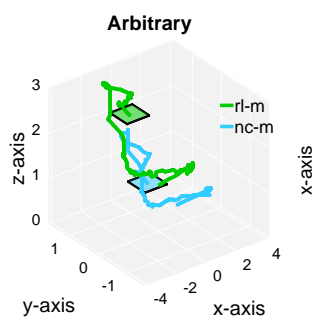

(m)

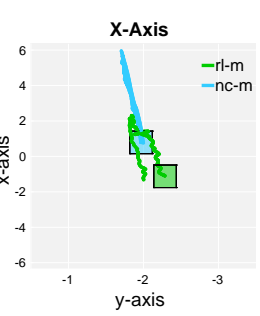

(b)

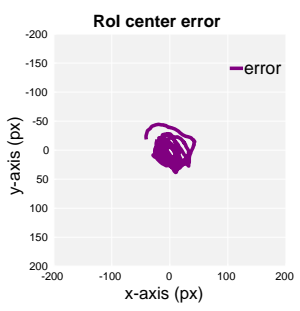

(c)

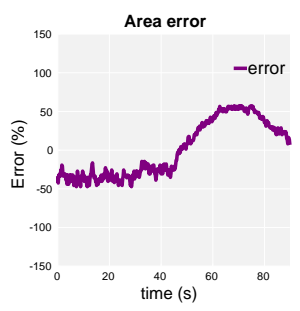

(d)

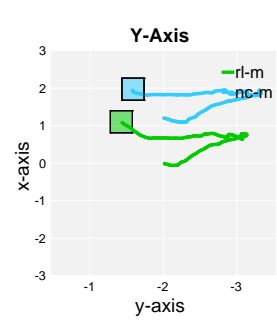

(f)

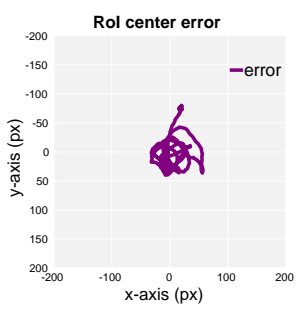

$(\mathrm{g})$

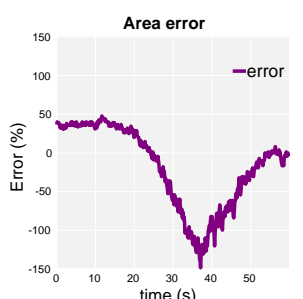

(h)

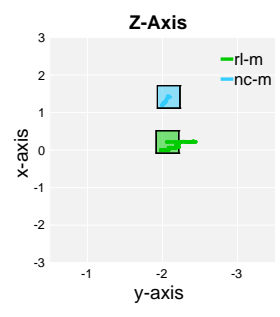

(j)

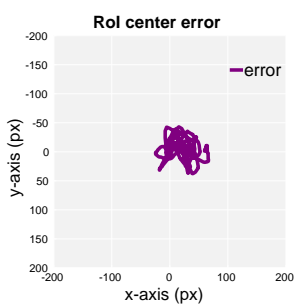

$(\mathrm{k})$

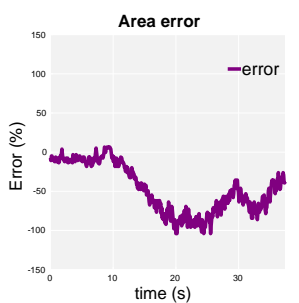

(1)

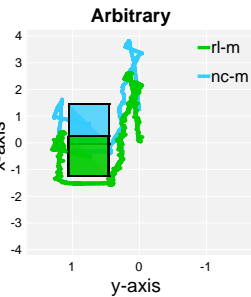

(n)

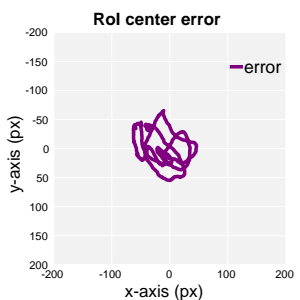

(o)

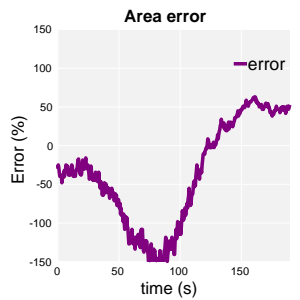

(p)

Figure 4.25: Real-flight results corresponding to four different scenarios for the P.II multirotor following approach. (a)(b)(e)(f)(i)(j) Rectilinear periodic trajectory of Noncooperative Multirotor (NC-M) along $\mathrm{x}, \mathrm{y}$, and $\mathrm{z}$ axes with a maximum velocity of $0.3 \mathrm{~m} / \mathrm{s}(X-$ Axis, $Y$-Axis and Z-Axis, respectively). (m)(n) Arbitrary nonperiodic trajectory of NC-M with maximum velocity of $0.3 \mathrm{~m} / \mathrm{s}($ Arbitrary). (c)(d)(g)(h)(k)(l)(o)(p) Current-target RoI center and area error corresponding to each of four stated experiments. Multirotor trajectories have been generated by an Extended Kalman Filter (EKF) that utilizes optical-flow velocities and measured accelerations as only input source.

this section is in accordance with C.5 - C.7 and fulfills objectives $\mathbf{O . 3}$ and O.5 - O.6. 


\subsubsection{Introduction}

Recent advances in image object detection have mainly focused on the development of CNN architectures Bochkovskiy et al. (2020); Liu et al. (2019b); Lin et al. (2017); Tan et al. (2019) to progressively increase accuracy or decrease processing times (see also P.II detection approach in Section 4.4.3. In this regard, accuracy has been the primary concern in the majority of studies Zhou et al. (2019); Redmon and Farhadi (2018); Girshick (2015); Lin et al. (2017), mostly due to the initial lack of techniques which precisely capture the variability found in the object detection task (number of classes, illumination and environmental ambiances, corner cases, etc.). Nevertheless, detection speed and power consumption are recently becoming key differentiator metrics Bochkovskiy et al. (2020); Huang et al. (2018); Gao et al. (2018); Pirinen and Sminchisescu (2018); Rungsuptaweekoon et al. (2017); Mao et al. (2016); Yu et al. (2018), as long as robotic solutions are being oriented to practical or industrial fields.

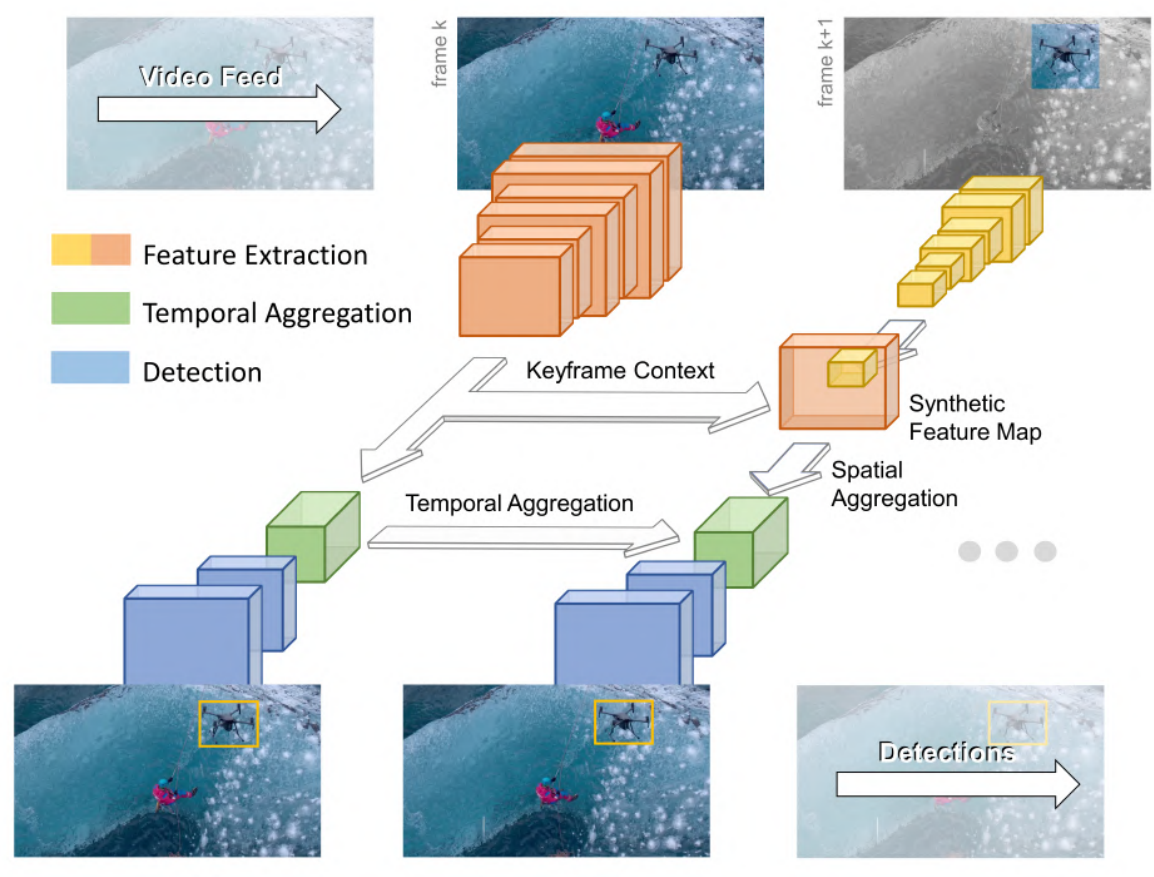

Figure 4.26: A schematic diagram of the P.III proposed approach. The keyframe context is dynamically reused to reduce computation overhead, and temporal information is propagated by the temporal aggregator. Grey region in an image frame denotes pixels which are not being processed for the given frame.

In the context of object detection techniques, the incoming images from video streams, commonly found in vision-based robotic applications, are treated separately with strategies designed for single-image processing (see Section 4.4.3). However, an important property of video is that adjacent video frames are highly correlated, which opens up the possibility of decreasing the computation latency. During the process of object detection in a sequence, the surrounding context of previous frame detection is prone to hold redundant content, which can be exploited in the current detection stage. Therefore, a simple idea is to keep a memory of previously extracted features and recompute only the ones corresponding to the region where the object was found in the previous frame. To follow this idea, features from the previous and current frames have to be fused in a synthetic feature map, which is used to compute the final detection. In this trend, based on the intuition that human 
peripheral vision may not add value while pursuing an object, P.III approach dynamically reuses feature context from previously detected frames to increase efficiency. The context is fed into a recurrent network structure for temporal aggregation. This concept is best illustrated in Fig. 4.26 .

Additionally, in P.III, it has been found that the aggregation of synthetic feature maps by the recurrent network structure contains within itself the information necessary to decide when the peripheral context has to be ignored. In this regard, an inattentional policy of when to use the full or synthetic feature map by formulating the task as a reinforcement learning problem has been included in order to increase the efficiency of the complete approach.

\subsubsection{Deep Learning Based Methods}

The P.III solution is approached as a dynamic context reuse across an image sequence $\mathcal{V}=\left\{I_{0}, I_{1}, I_{2}, \ldots, I_{n}\right\}$. Indeed, the phenomenon of momentarily ignoring context, while detecting an object, can be effectively reformulated as a context reuse, since reusing previous context is equivalent to avoiding current context computation. Besides, our framework is restricted to the online setting where only $\left\{I_{0}, I_{1}, \ldots, I_{k}\right\}$ are available during the computation of the $k$-th detection.

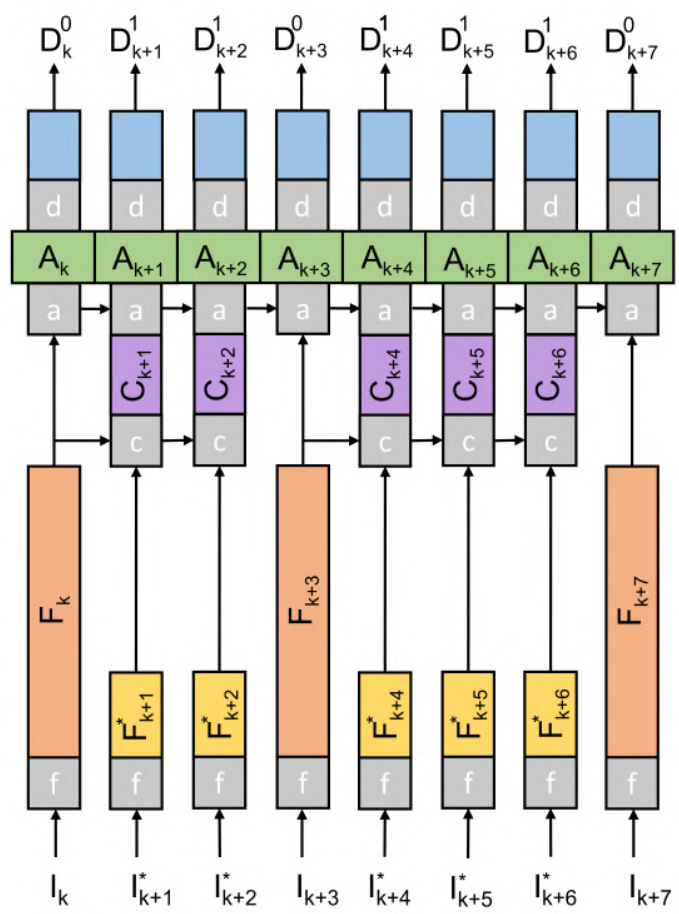

Figure 4.27: A block diagram of our adaptive keyframe selection technique with all the components of the framework included, where $f$ stands for feature extractor, $c$ is the context aggregator, $a$ depicts the temporal aggregator, and $d$ represents the detector. For clarity, coloured blocks correspond to tensors and grey blocks correspond to components of the system. The length of the tensor denotes time consumption but it is not to scale. The context of a keyframe $F_{k}^{c}$ is not being modified throughout the pipeline of context aggregators.

To materialize this concept into the DL paradigm, four main components have been defined in the framework: a feature extractor $\mathbf{f}$, a context aggregator $\mathbf{c}$, a temporal aggregator 
$\mathbf{a}$, and a detector $\mathbf{d}$. The context is defined as a subset of the feature maps generated by the feature extractor $\mathbf{f}$ on a keyframe $k$. In this direction, the approach interleaves full keyframe computation and partial frame (inattentional frame) computation with context aggregation (see Fig. 4.27) to provide an intermediate representation. The frame-level features are then temporally aggregated and refined using a recurrent architecture. Finally, an SSD-style Sandler et al. (2018) detection pipeline is applied to the refined feature map to obtain bounding box results. For a detailed explanation of the framework, the reader is encouraged to revise P.III.

\subsubsection{Reinforcement Learning Based Methods}

The process of interleaving full keyframe and partial frame computation (inattentional frame) can be carried in a naive trend (i.e. randomly selecting full keyframe computation). Nevertheless, P.III explored the possibility of performing the keyframe selection in an adaptive trend. On this subject, the state information of the temporal aggregator component has been utilized as the input state of a reinforcement learning agent, which is encouraged to perform the optimal amount of full keyframe computation.

In this regard, the agent has been encouraged, through the reward function of the reinforcement learning formulation, to perform the minimum amount of full keyframe processing, though maintaining accuracy. The reward function definition has been the following:

$$
r= \begin{cases}\min _{i}\left(L\left(D^{i}\right)\right)-L\left(D^{0}\right) & a=0 \\ \psi+\min _{i}\left(L\left(D^{i}\right)\right)-L\left(D^{1}\right) & a=1\end{cases}
$$

where $D^{0}$ and $D^{1}$ are the detection results through the expensive pipeline (full image) and cheap pipeline (context aggregation), respectively; $L(\cdot)$ is the Multibox loss Liu et al. (2016a) computation and $\psi$ corresponds to the inattentional factor. The inattentional factor $\psi$ is an important component of the reward function. It is a scalar value that can potentially encourage the agent to take the cheap pipeline, even when its cost remains higher. The definition of the $\psi$ value is decisive for the final behavior of the agent since it modulates the amount of accuracy the agent is able to forfeit in favor of latency reduction.

Furthermore, it is not trivial to select the inattentional factor value, since it is strongly coupled to the application under research. There can be applications where the most important requirement is latency, being able to provide a reduced accuracy, or on the contrary, the accuracy can be crucial and not the latency. In this context, You Only Train Once (YOTO) technique Dosovitskiy and Djolonga (2019), which was primarily designed to DL-based supervised and unsupervised training schemes, has been extended in P.III to the reinforcement learning paradigm in order to provide the agent the novel ability to encapsulate not only a unique behavior based on a reward function but a whole distribution of behaviors based on a distribution of reward functions. The precise technique has been detailed in Dosovitskiy and Djolonga (2019) and P.III. For the application under study, where the agent is meant to adaptively select keyframe and partial frame computation, YOTO technique has been applied to the inattentional factor, to condition the agent to any inattentional factor at inference time. The resulting reward function is the following:

$$
r\left(\lambda_{0}\right)= \begin{cases}\min _{i}\left(L\left(D^{i}\right)\right)-L\left(D^{0}\right) & a=0 \\ \lambda_{0}+\min _{i}\left(L\left(D^{i}\right)\right)-L\left(D^{1}\right) & a=1\end{cases}
$$

where $\psi=\lambda_{0} \sim P_{\lambda}$ and $P_{\lambda}$ denotes a $\log$ uniform distribution of probability with 
$\lambda_{0} \in[0,2]$. The PPO agent has been trained over 6321 update steps, with trajectories of 128 experiences (approximately over $20 \mathrm{~h}$ ). The best policy network has been automatically selected during training based on the maximum accuracy improvement over a random baseline (for the same number of inattentional frames).

In P.III, several experiments have been carried out, for two types of backbone architectures (MobileNetV1 Howard et al. (2017), and MobileNetV2 Sandler et al. (2018)) and two types of datasets, the Imagenet VID 2015 Russakovsky et al. (2015) and a custom UAV VID dataset (MAV-VID). The custom MAV-VID dataset has been made publicly available ${ }^{11}$ In addition, four values for $\lambda_{0} \in[0.01,0.5,1.2,2.0]$ have been tested. The standard Imagenet VID accuracy metric is reported, mean Average Precision mAP@0.5 IOU. The latency of the networks in milliseconds (ms) and the average Frames per Second (FPS) of the complete approach are provided. The number of parameters is reported as benchmarks for efficiency. Also, the power consumption in Watts (W) and the energy efficiency in FPS/W are included for every experiment, as reported in Rungsuptaweekoon et al. (2017); Mao et al. (2016); Yu et al. (2018). The validation tests have been performed in two different GPUs, an Nvidia GeForce RTX 2080 Ti desktop GPU and an Nvidia Volta embedded GPU inside an Nvidia Jetson AGX Xavier platform. Also, since the latency reduction greatly stands out when the parallelization capacity is decreased, such as the case of desktop and embedded CPUs, the proposed system has been further tested and validated in an Intel Core i7-9700K@3.60GHz desktop CPU and a ARMv8.2@1.377GHz embedded CPU. The code has been made publicly available ${ }^{12}$ and a short video demonstration has been provided 13

\begin{tabular}{|c|c|c|c|c|c|c|c|c|c|}
\hline Model & Platform & mAP & $\begin{array}{l}\mathrm{KF} \\
(\mathrm{ms})\end{array}$ & $\begin{array}{l}\mathrm{IF} \\
(\mathrm{ms})\end{array}$ & FPS & $\begin{array}{l}\text { FPS } \\
\text { Ratio }\end{array}$ & $\begin{array}{l}\text { Params } \\
(\mathrm{M})\end{array}$ & $\begin{array}{l}\text { Power } \\
(\mathrm{W})\end{array}$ & $\begin{array}{l}\text { Efficiency } \\
(\mathrm{FPS} / \mathrm{W})\end{array}$ \\
\hline MobileNetV1-SSDLite & $\begin{array}{l}\text { GPU (Desktop) } \\
\text { GPU (Xavier) } \\
\text { CPU (Desktop) } \\
\text { CPU (Xavier) }\end{array}$ & 0.4824 & $\begin{array}{l}20.67 \\
84.53 \\
159.73 \\
7018.31 \\
\end{array}$ & $\begin{array}{l}- \\
- \\
- \\
-\end{array}$ & $\begin{array}{l}48.37 \\
11.83 \\
26.26 \\
0.142\end{array}$ & $\begin{array}{l}- \\
- \\
- \\
-\end{array}$ & 4.48 & $\begin{array}{l}128.3 \\
4.19 \\
99.1 \\
2.91 \\
\end{array}$ & $\begin{array}{l}0.377 \\
2.82 \\
0.264 \\
0.048\end{array}$ \\
\hline $\begin{array}{l}\text { MobileNetV1-ConvLSTM- } \\
\text { SSDLite }\end{array}$ & $\begin{array}{l}\text { GPU (Desktop) } \\
\text { GPU (Xavier) } \\
\text { CPU (Desktop) } \\
\text { CPU (Xavier) }\end{array}$ & 0.5236 & \begin{tabular}{l|}
19.33 \\
83.73 \\
39.53 \\
6378.85 \\
\end{tabular} & $\begin{array}{l}- \\
- \\
- \\
-\end{array}$ & $\begin{array}{l}51.73 \\
11.94 \\
25.29 \\
0.156 \\
\end{array}$ & $\begin{array}{l}- \\
- \\
- \\
-\end{array}$ & 3.3 & $\begin{array}{l}126.8 \\
3.82 \\
98.9 \\
2.87 \\
\end{array}$ & $\begin{array}{l}0.407 \\
3.12 \\
0.255 \\
0.054 \\
\end{array}$ \\
\hline $\begin{array}{l}\text { MobileNetV1-ConvLSTM- } \\
\text { SSDLite }\left[\lambda_{0}=0.01\right]\end{array}$ & $\begin{array}{l}\text { GPU (Desktop) } \\
\text { GPU (Xavier) } \\
\text { CPU (Desktop) } \\
\text { CPU (Xavier) }\end{array}$ & 0.5235 & $\begin{array}{l}19.59 \\
83.33 \\
39.62 \\
6512.13\end{array}$ & \begin{tabular}{l|}
18.78 \\
76.63 \\
25.01 \\
4132.13
\end{tabular} & $\begin{array}{l}51.91 \\
12.4 \\
29.65 \\
0.1801\end{array}$ & $\begin{array}{l}1.0 \\
1.03 \\
1.17 \\
1.15\end{array}$ & 5.5 & $\begin{array}{l}141.5 \\
4.25 \\
103.1 \\
3.03\end{array}$ & $\begin{array}{l}0.366 \\
2.91 \\
0.287 \\
0.059\end{array}$ \\
\hline $\begin{array}{l}\text { MobileNetV1-ConvLSTM- } \\
\text { SSDLite }\left[\lambda_{0}=1.2\right]\end{array}$ & $\begin{array}{l}\text { GPU (Desktop) } \\
\text { GPU (Xavier) } \\
\text { CPU (Desktop) } \\
\text { CPU (Xavier) }\end{array}$ & 0.4781 & $\begin{array}{l}19.99 \\
83.1 \\
39.33 \\
6580.88\end{array}$ & \begin{tabular}{l|}
18.68 \\
75.15 \\
25.07 \\
4063.28
\end{tabular} & $\begin{array}{l}52.85 \\
13.01 \\
36.11 \\
0.2209\end{array}$ & $\begin{array}{l}1.0 \\
1.08 \\
1.42 \\
1.41\end{array}$ & 5.5 & $\begin{array}{l}141.5 \\
4.25 \\
103.1 \\
3.03\end{array}$ & $\begin{array}{l}0.373 \\
3.06 \\
0.35 \\
0.072\end{array}$ \\
\hline $\begin{array}{l}\text { MobileNetV1-ConvLSTM- } \\
\text { SSDLite }\left[\lambda_{0}=2.0\right]\end{array}$ & $\begin{array}{l}\text { GPU (Desktop) } \\
\text { GPU (Xavier) } \\
\text { CPU (Desktop) } \\
\text { CPU (Xavier) }\end{array}$ & 0.4373 & $\begin{array}{l}19.91 \\
83.46 \\
39.89 \\
6593.12\end{array}$ & $\begin{array}{l}18.23 \\
76.36 \\
25.45 \\
4028.2\end{array}$ & $\begin{array}{l}54.48 \\
13.15 \\
37.73 \\
0.2372\end{array}$ & $\begin{array}{l}1.05 \\
1.1 \\
1.49 \\
1.52\end{array}$ & 5.5 & $\begin{array}{l}141.5 \\
4.25 \\
103.1 \\
3.03\end{array}$ & $\begin{array}{l}0.385 \\
3.09 \\
0.365 \\
0.078\end{array}$ \\
\hline
\end{tabular}

Table 4.2: Results using the MobileNetV1 feature extractor on the ImageNet VID 2015 dataset.

In Table 4.2 and Table 4.3 , the results corresponding to MobileNetV1 feature extractor

\footnotetext{
${ }^{11}$ https://www.kaggle.com/alejodosr/multirotor-aerial-vehicle-vid-mavvid-dataset

12 https://github.com/alejodosr/adaptive-inattention

13 https://vimeo.com/426725929
} 


\begin{tabular}{|c|c|c|c|c|c|c|c|c|}
\hline Model & Platform & $\mathrm{mAP}$ & $\begin{array}{l}\mathrm{KF} \\
(\mathrm{ms})\end{array}$ & $\begin{array}{l}\mathrm{IF} \\
(\mathrm{ms})\end{array}$ & FPS & $\begin{array}{l}\text { FPS } \\
\text { Ratio }\end{array}$ & $\begin{array}{l}\text { Power } \\
(\mathrm{W})\end{array}$ & $\begin{array}{l}\text { Efficiency } \\
\text { (FPS/W) }\end{array}$ \\
\hline \multirow{4}{*}{ MobileNetV1-SSDLite } & GPU (Desktop) & \multirow{4}{*}{0.9183} & 18.85 & - & 53.05 & - & 128.2 & 0.413 \\
\hline & GPU (Xavier) & & 90.66 & - & 11.03 & - & 4.16 & 2.65 \\
\hline & CPU (Desktop) & & 122.58 & - & 8.15 & - & 99.0 & 0.082 \\
\hline & CPU (Xavier) & & 6406.07 & - & 0.156 & - & 2.93 & 0.053 \\
\hline \multirow{4}{*}{$\begin{array}{l}\text { MobileNetV1-ConvLSTM- } \\
\text { SSDLite }\end{array}$} & GPU (Desktop) & \multirow{4}{*}{0.9398} & 17.84 & - & 56.05 & - & 126.7 & 0.442 \\
\hline & GPU (Xavier) & & 85.54 & - & 11.69 & - & 3.79 & 3.08 \\
\hline & CPU (Desktop) & & 85.75 & - & 11.66 & - & 99.0 & 0.117 \\
\hline & CPU (Xavier) & & 5375.18 & - & 0.186 & - & 2.91 & 0.063 \\
\hline \multirow{4}{*}{$\begin{array}{l}\text { MobileNetV1-ConvLSTM- } \\
\text { SSDLite }\left[\lambda_{0}=0.01\right]\end{array}$} & GPU (Desktop) & \multirow{4}{*}{0.9382} & 17.89 & 16.12 & 55.91 & 0.99 & 141.4 & 0.395 \\
\hline & GPU (Xavier) & & 85.58 & 70.78 & 11.69 & 1.0 & 4.19 & 2.83 \\
\hline & CPU (Desktop) & & 85.81 & 27.62 & 11.68 & 1.0 & 103.0 & 0.116 \\
\hline & CPU (Xavier) & & 5354.33 & 3967.07 & 0.1869 & 1.0 & 3.15 & 0.061 \\
\hline \multirow{4}{*}{$\begin{array}{l}\text { MobileNetV1-ConvLSTM- } \\
\text { SSDLite }\left[\lambda_{0}=0.5\right]\end{array}$} & GPU (Desktop) & \multirow{4}{*}{0.9377} & 17.95 & 16.38 & 55.93 & 0.99 & 141.4 & 0.395 \\
\hline & GPU (Xavier) & & 84.77 & 71.2 & 11.88 & 1.01 & 4.19 & 2.78 \\
\hline & CPU (Desktop) & & 85.73 & 26.94 & 12.03 & 1.03 & 103.0 & 0.116 \\
\hline & CPU (Xavier) & & 5256.23 & 3845.12 & 0.1925 & 1.03 & 3.15 & 0.061 \\
\hline \multirow{4}{*}{$\begin{array}{l}\text { MobileNetV1-ConvLSTM- } \\
\text { SSDLite }\left[\lambda_{0}=1.2\right]\end{array}$} & GPU (Desktop) & \multirow{4}{*}{0.8845} & 17.96 & 16.39 & 58.41 & 1.04 & 141.4 & 0.413 \\
\hline & GPU (Xavier) & & 85.21 & 71.04 & 12.88 & 1.1 & 4.19 & 3.07 \\
\hline & CPU (Desktop) & & 85.9 & 27.91 & 18.25 & 1.56 & 103.0 & 0.177 \\
\hline & CPU (Xavier) & & 5312.47 & 3834.12 & 0.2212 & 1.18 & 3.15 & 0.07 \\
\hline \multirow{4}{*}{$\begin{array}{l}\text { MobileNetV1-ConvLSTM- } \\
\text { SSDLite }\left[\lambda_{0}=2.0\right]\end{array}$} & GPU (Desktop) & \multirow{4}{*}{0.8403} & 17.96 & 16.68 & 58.86 & 1.05 & 141.4 & 0.416 \\
\hline & GPU (Xavier) & & 86.58 & 70.99 & 13.37 & 1.14 & 4.19 & 3.19 \\
\hline & CPU (Desktop) & & 85.8 & 26.74 & 24.39 & 2.09 & 103.0 & 0.236 \\
\hline & CPU (Xavier) & & 5214.32 & 3956.01 & 0.234 & 1.25 & 3.15 & 0.074 \\
\hline
\end{tabular}

Table 4.3: Results using the MobileNetV1 feature extractor on the MAV-VID dataset.

\begin{tabular}{|c|c|c|c|c|c|c|c|c|c|}
\hline Model & Platform & $\mathrm{mAP}$ & $\begin{array}{l}\mathrm{KF} \\
(\mathrm{ms})\end{array}$ & $\begin{array}{l}\text { IF } \\
(\mathrm{ms})\end{array}$ & FPS & $\begin{array}{l}\text { FPS } \\
\text { Ratio }\end{array}$ & $\begin{array}{l}\text { Params } \\
(\mathrm{M})\end{array}$ & $\begin{array}{l}\text { Power } \\
(\mathrm{W})\end{array}$ & $\begin{array}{l}\text { Efficiency } \\
\text { (FPS/W }\end{array}$ \\
\hline \multirow{4}{*}{ MobileNetV2-SSDLite } & GPU (Desktop) & \multirow{4}{*}{0.4775} & 35.79 & - & 27.94 & - & \multirow{4}{*}{4.84} & 127.3 & 0.219 \\
\hline & GPU (Xavier) & & 117.34 & - & 8.52 & - & & 4.31 & 1.97 \\
\hline & CPU (Desktop) & & 297.88 & - & 3.35 & - & & 98.2 & 0.034 \\
\hline & CPU (Xavier) & & 9001.32 & - & 0.1111 & - & & 3.01 & 0.036 \\
\hline \multirow{4}{*}{$\begin{array}{l}\text { MobileNetV2-ConvLSTM- } \\
\text { SSDLite }\end{array}$} & GPU (Desktop) & \multirow{4}{*}{0.4924} & 36.91 & - & 27.09 & - & \multirow{4}{*}{3.3} & 128.4 & 0.21 \\
\hline & GPU (Xavier) & & 126.82 & - & 7.7 & - & & 5.03 & 1.53 \\
\hline & CPU (Desktop) & & 261.62 & - & 3.82 & - & & 99.1 & 0.038 \\
\hline & CPU (Xavier) & & 8822.3 & - & 0.1133 & - & & 3.12 & 0.036 \\
\hline \multirow{4}{*}{$\begin{array}{l}\text { MobileNetV2-ConvLSTM- } \\
\text { SSDLite }\left[\lambda_{0}=0.01\right]\end{array}$} & GPU (Desktop) & \multirow{4}{*}{0.491} & 35.64 & 34.81 & 28.14 & 1.03 & \multirow{4}{*}{5.5} & 142.5 & 0.197 \\
\hline & GPU (Xavier) & & 126.66 & 118.53 & 7.96 & 1.03 & & 5.17 & 1.53 \\
\hline & CPU (Desktop) & & 263.126 & 190.95 & 3.94 & 1.03 & & 102.9 & 0.038 \\
\hline & CPU (Xavier) & & 8852.42 & 6267.98 & 0.1176 & 1.03 & & 3.23 & 0.036 \\
\hline \multirow{4}{*}{$\begin{array}{l}\text { MobileNetV2-ConvLSTM- } \\
\text { SSDLite }\left[\lambda_{0}=0.5\right]\end{array}$} & GPU (Desktop) & \multirow{4}{*}{0.4818} & 36.83 & 34.39 & 28.03 & 1.03 & \multirow{4}{*}{5.5} & 142.5 & 0.196 \\
\hline & GPU (Xavier) & & 126.32 & 118.79 & 8.14 & 1.05 & & 5.17 & 1.57 \\
\hline & CPU (Desktop) & & 264.02 & 163.84 & 4.62 & 1.2 & & 102.9 & 0.044 \\
\hline & CPU (Xavier) & & 8752.36 & 6189.12 & 0.1312 & 1.15 & & 3.23 & 0.04 \\
\hline \multirow{4}{*}{$\begin{array}{l}\text { MobileNetV2-ConvLSTM- } \\
\text { SSDLite }\left[\lambda_{0}=1.2\right]\end{array}$} & GPU (Desktop) & \multirow{4}{*}{0.4704} & 36.67 & 34.71 & 28.24 & 1.04 & \multirow{4}{*}{5.5} & 142.5 & 0.198 \\
\hline & GPU (Xavier) & & 126.08 & 119.1 & 8.22 & 1.06 & & 5.17 & 1.58 \\
\hline & CPU (Desktop) & & 264.14 & 152.34 & 5.2 & 1.36 & & 102.9 & 0.05 \\
\hline & CPU (Xavier) & & 8952.78 & 6104.78 & 0.1406 & 1.24 & & 3.23 & 0.043 \\
\hline \multirow{4}{*}{$\begin{array}{l}\text { MobileNetV2-ConvLSTM- } \\
\text { SSDLite }\left[\lambda_{0}=2.0\right]\end{array}$} & GPU (Desktop) & \multirow{4}{*}{0.4622} & 36.08 & 34.19 & 28.89 & 1.06 & \multirow{4}{*}{5.5} & 142.5 & 0.202 \\
\hline & GPU (Xavier) & & 126.25 & 118.68 & 8.3 & 1.07 & & 5.17 & 1.6 \\
\hline & CPU (Desktop) & & 264.22 & 144.99 & 5.83 & 1.52 & & 102.9 & 0.056 \\
\hline & CPU (Xavier) & & 8876.23 & 6123.43 & 0.1485 & 1.31 & & 3.23 & 0.045 \\
\hline
\end{tabular}

Table 4.4: Results using the MobileNetV2 feature extractor on the ImageNet VID 2015 dataset.

for both Imagenet VID 2015 and MAV-VID dataset are shown. In addition, in Table 4.4 and Table 4.5, the results corresponding to MobileNetV2 feature extractor for both Imagenet VID 2015 and MAV-VID dataset are also depicted.

In order to further validate the performance of the proposed inattentional policy, a 


\begin{tabular}{|c|c|c|c|c|c|c|c|c|}
\hline Model & Platform & $\mathrm{mAP}$ & $\begin{array}{l}\mathrm{KF} \\
(\mathrm{ms})\end{array}$ & $\begin{array}{l}\text { IF } \\
(\mathrm{ms})\end{array}$ & FPS & $\begin{array}{l}\text { FPS } \\
\text { Ratio } \\
\end{array}$ & $\begin{array}{l}\text { Power } \\
(\mathrm{W})\end{array}$ & $\begin{array}{l}\text { Efficiency } \\
(\text { FPS/W) }\end{array}$ \\
\hline \multirow{4}{*}{ MobileNetV2-SSDLite } & GPU (Desktop) & \multirow{4}{*}{0.9218} & 25.819 & - & 38.73 & - & 127.4 & 0.304 \\
\hline & GPU (Xavier) & & 120.82 & - & 8.27 & - & 4.32 & 1.91 \\
\hline & CPU (Desktop) & & 222.92 & - & 4.48 & - & 98.1 & 0.045 \\
\hline & CPU (Xavier) & & 7645.68 & - & 0.1308 & - & 3.16 & 0.041 \\
\hline \multirow{4}{*}{$\begin{array}{l}\text { MobileNetV2-ConvLSTM- } \\
\text { SSDLite }\end{array}$} & GPU (Desktop) & \multirow{4}{*}{0.9453} & 29.78 & - & 33.57 & - & 128.5 & 0.261 \\
\hline & GPU (Xavier) & & 123.15 & - & 8.12 & - & 5.14 & 1.57 \\
\hline & CPU (Desktop) & & 230.7 & - & 4.33 & - & 99.2 & 0.043 \\
\hline & CPU (Xavier) & & 8002.68 & - & 0.125 & - & 3.13 & 0.039 \\
\hline \multirow{4}{*}{$\begin{array}{l}\text { MobileNetV2-ConvLSTM- } \\
\text { SSDLite }\left[\lambda_{0}=0.01\right]\end{array}$} & GPU (Desktop) & \multirow{4}{*}{0.9453} & 29.2 & - & 34.24 & 1.02 & 142.6 & 0.24 \\
\hline & GPU (Xavier) & & 123.02 & - & 8.12 & 1.0 & 5.11 & 1.58 \\
\hline & CPU (Desktop) & & 231.25 & - & 4.32 & 1.0 & 102.8 & 0.042 \\
\hline & CPU (Xavier) & & 7903.04 & - & 0.1265 & 1.0 & 3.13 & 0.04 \\
\hline \multirow{4}{*}{$\begin{array}{l}\text { MobileNetV2-ConvLSTM- } \\
\text { SSDLite }\left[\lambda_{0}=0.5\right]\end{array}$} & GPU (Desktop) & \multirow{4}{*}{0.9452} & 29.13 & 28.14 & 34.33 & 1.02 & 142.6 & 0.24 \\
\hline & GPU (Xavier) & & 122.47 & 115.23 & 8.16 & 1.0 & 5.11 & 1.59 \\
\hline & CPU (Desktop) & & 230.91 & 50.75 & 4.33 & 1.0 & 102.8 & 0.042 \\
\hline & CPU (Xavier) & & 7877.87 & 3319.54 & 0.127 & 1.01 & 3.13 & 0.04 \\
\hline \multirow{4}{*}{$\begin{array}{l}\text { MobileNetV2-ConvLSTM- } \\
\text { SSDLite }\left[\lambda_{0}=1.2\right]\end{array}$} & GPU (Desktop) & \multirow{4}{*}{0.86} & 29.08 & 28.47 & 34.78 & 1.03 & 142.6 & 0.243 \\
\hline & GPU (Xavier) & & 123.11 & 115.1 & 8.41 & 1.03 & 5.11 & 1.64 \\
\hline & CPU (Desktop) & & 232.09 & 51.77 & 7.43 & 1.71 & 102.8 & 0.07 \\
\hline & CPU (Xavier) & & 8009.22 & 3243.22 & 0.1842 & 1.47 & 3.13 & 0.058 \\
\hline \multirow{4}{*}{$\begin{array}{l}\text { MobileNetV2-ConvLSTM- } \\
\text { SSDLite }\left[\lambda_{0}=2.0\right]\end{array}$} & GPU (Desktop) & \multirow{4}{*}{0.8364} & 28.88 & 28.49 & 34.93 & 1.04 & 142.6 & 0.244 \\
\hline & GPU (Xavier) & & 123.15 & 114.65 & 8.51 & 1.04 & 5.11 & 1.66 \\
\hline & CPU (Desktop) & & 232.04 & 51.22 & 8.92 & 2.06 & 102.8 & 0.086 \\
\hline & CPU (Xavier) & & 7865.12 & 3500.33 & 0.2013 & 1.61 & 3.13 & 0.064 \\
\hline
\end{tabular}

Table 4.5: Results using the MobileNetV2 feature extractor on the MAV-VID dataset.

comparison with a random baseline for a wide variety of scenarios has been carried out. In Fig. 4.28 the trade-off between the number of inattentional frames executed and the resulting $\mathrm{mAP}$ is illustrated for both datasets and feature extractors. For every value of the reward-conditional parameter $\lambda_{0}$, there is an overall percentage of inattentional frames executed. In order to be able to compare it to a random baseline, a random policy has been executed the same exact amount of inattentional frames. Also, the original mAP (with no inattentional frames involved) is included. Every test has been performed 5 times each, for both policies, and the average results have been plotted.

Finally, four application cases have been illustrated in Fig. 4.29. Two complex scenarios, due to object high-motion speed within the image plane or environmental complexity (camera pointing to the sun); and two simple (or static) scenarios, where the object stays almost static within the image plane, have been additionally depicted.

Regarding Table 4.2, 4.3, 4.4, and 4.5, both architectures provide state-of-the-art mAP at $0.5 \mathrm{IOU}$ in the Imagenet VID 2015 dataset, resulting in 0.5236 and 0.4924 for MobileNetV1 and MobileNetV2 feature extractors, respectively. Also, the mAP results in our custom MAV-VID dataset are notable, with 0.9398 and 0.9453 for MobileNetV1 and MobileNetV2 feature extractors, respectively. MobileNetV1 provided higher mAP in Imagenet VID 2015 dataset, whereas MobileNetV2 yielded higher mAP in the MAV-VID dataset.

The mAP gets reduced when the reward-conditional parameter $\lambda_{0}$ increases since the policy is encouraged to execute more inattentional frames at the cost of accuracy. Nevertheless, it maintains competitive values throughout the whole range, with a minimum accuracy of 0.4373 and 0.8403 for Imagenet VID 2015 and MAV-VID dataset, respectively. As shown in Table 4.4 and 4.5, MobileNetV2-ConvLSTM-SSDLite has remained as the model with lower mAP degradation.

The proposed inattentional framework has achieved a considerable latency reduction, increasing the runtime FPS in every platform tested, with minimal accuracy drop. Never- 


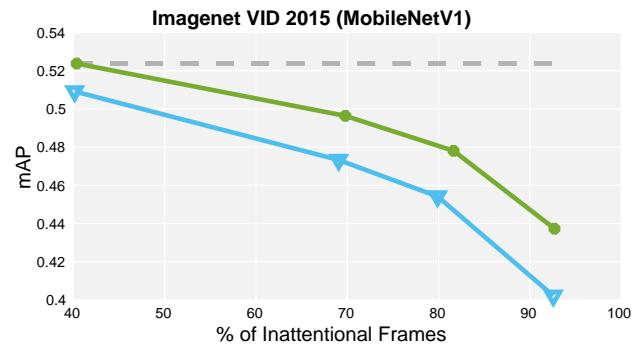

(a)

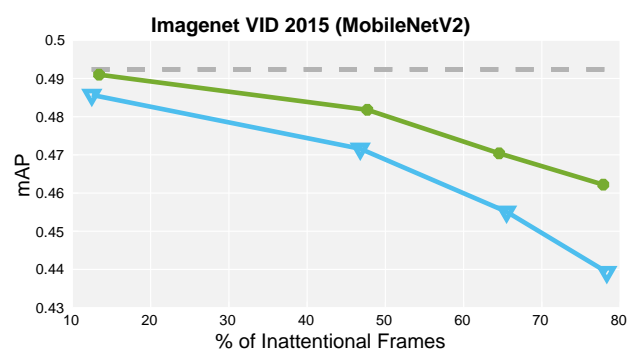

(c)

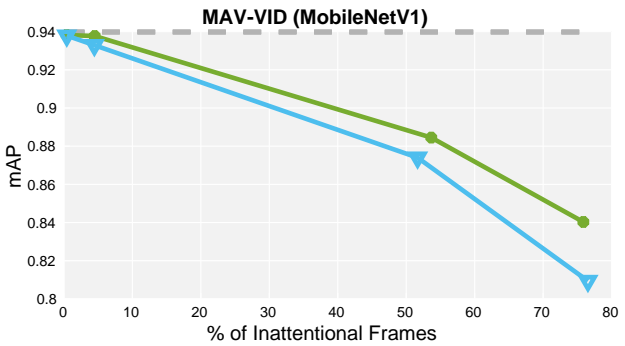

(b)

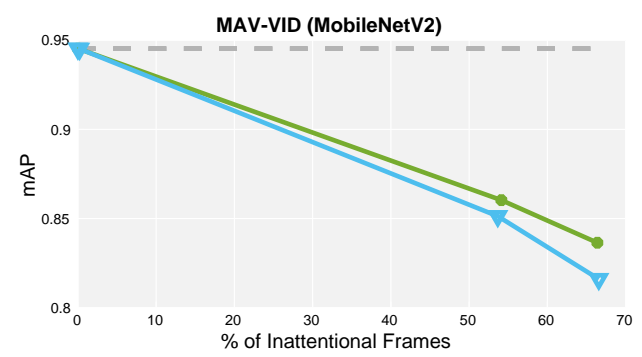

(d)

Figure 4.28: Representation of the trade-off between the mAP and the percentage of inattentional frames executed. Four cases with two feature extractors (MobileNetV1 and MobileNetV2) and two datasets (Imagenet VID 2015 and MAV-VID) have been evaluated. The random baseline policy is depicted in pale blue (circles) and the proposed inattentional policy in pale green (triangles). The original mAP (with no inattentional frames involved) is represented with a grey dashed line. The inattentional policy predominates allover the tests, resulting in a higher mAP for every percentage of inattentional frames. Also, the inattentional policy incurs less accuracy degradation even at extreme ratios of inattentional frames, suggesting that P.III method is superior at capturing the temporal dynamics inherent to videos. Every test has been performed 5 times each, for both policies, and the average results have been plotted.

theless, the amount of computation reduction is highly dependent on the platform where the system is executed, as well as on the average object size within the image plane. Considering the GPU platforms, with a higher parallelization capacity, the average FPS increase ratio has ranged from 1.0 to 1.14 for MobileNetV1 and from 1.0 to 1.07 for MobileNetV2. However, regarding the CPU platforms, where the parallelization capacity is limited, the average FPS increase ratio has ranged from 1.0 to 2.09 for MobileNetV1 and from 1.0 to 2.06 for MobileNetV2. These results lead to an average FPS on the desktop CPU platform of 37.73, which is in the order of magnitude of the base runtime FPS on the desktop GPU platform of 51.91, at the cost of $0.086 \mathrm{mAP}$ reduction, for MobileNetV1 in Imagenet VID 2015 dataset. The maximum FPS increase ratio has been 2.09 for MobileNetV1 on the desktop CPU, achieving a runtime FPS of 24.39 at the cost of $0.09 \mathrm{mAP}$ reduction, in MAV-VID dataset (where the object size is on average smaller in the image plane). These results suggest that the P.III framework is able to increase computation efficiency when the effective parallelization capacity is limited. The effective parallelization capacity is influenced by several variables and it is relative to the input images, the model size, and the platform specifications. Qualitatively, parallelization is low in average CPUs, in appli- 


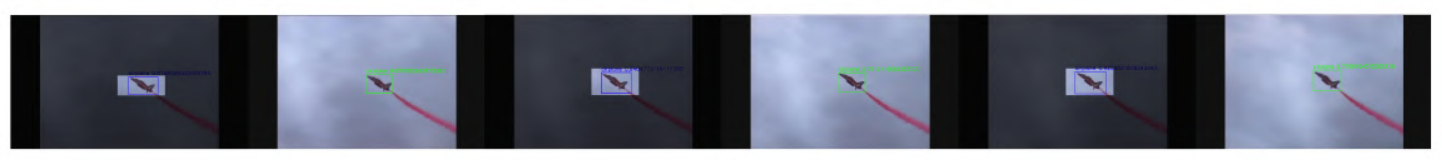

(a)

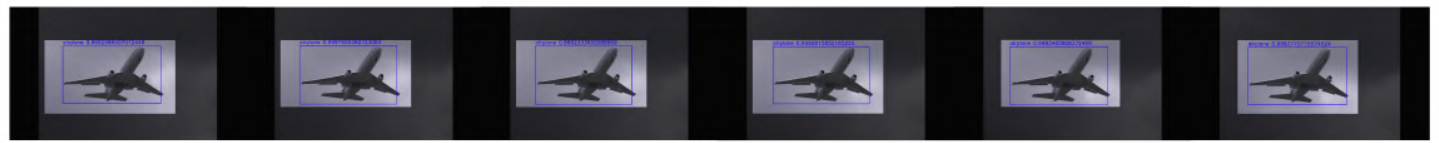

(b)

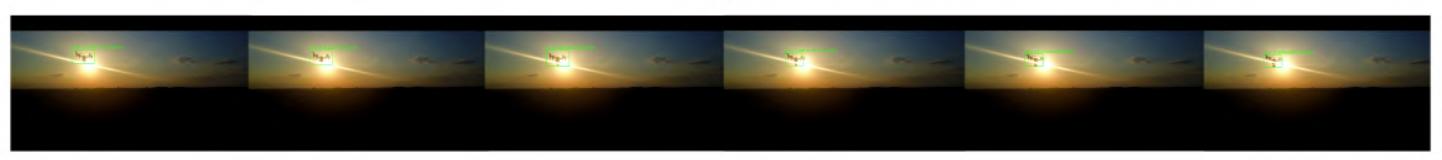

(c)

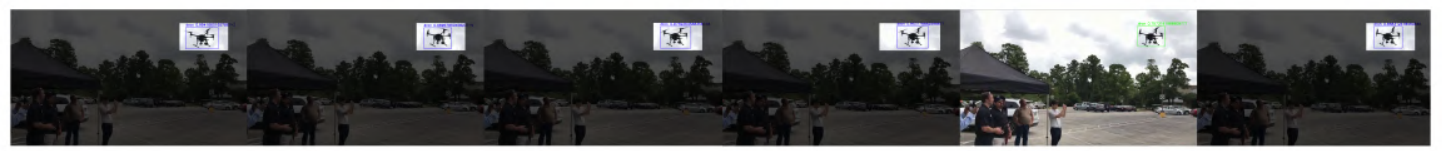

(d)

Figure 4.29: Images corresponding to four different scenarios for both datasets. Every image example has been processed by MobileNetV1-ConvLSTM-SSDLite $\left[\lambda_{0}=1.2\right]$ model (MobileNetV2-ConvLSTM-SSDLite provides a similar behavior). Shaded regions correspond to non-processed pixels. (a)-(b) and (c)-(d) image examples correspond to Imagenet VID 2015 and MAV-VID dataset, respectively. (a) and (c) show complex detection examples, corresponding to an object with high-motion speed within the image plane, and to an increased environmental complexity (camera pointing to the sun), respectively. In this context, the inattentional policy executes a lower percentage of inattentional frames. (b) and (d) illustrate objects which remain more static throughout the sequence. In this scenario, the number of inattentional frames is higher.

cations where the input images are at high resolution or in applications where the models have notably more parameters than our MobileNetV1/V2-ConvLSTM-SSDLite models. These facts yield to an open field of research, where the proposed inattentional framework can be applied. Nevertheless, such an extensive study was out of the scope of the P.III work.

Regarding power consumption and energy efficiency, the proposed inattentional framework provides proper results in terms of both global power consumption and energy efficiency increase. Power consumption is notably higher in desktop GPU/CPUs, with on average 35x more power consumption than the embedded Xavier platform. The lowest energy consumption has been $2.87 \mathrm{~W}$ for MobileNetV1-ConvLSTM-SSDLite base model (no inattentional policy) in Imagenet VID 2015 dataset. Energy efficiency varies across platforms, being the GPUs more energy efficient due to their parallelization capabilities. The maximum energy efficiency has resulted in 3.19 FPS/W (1.12x) for MobileNetV1 on the Xavier GPU, achieving a runtime FPS of 13.37 at the cost of $4.19 \mathrm{~W}$, in MAV-VID dataset. It has to be noted that, regarding relative energy efficiency increase with respect to the base energy efficiency (no inattentional frames), the results follow approximately the same ratios as in the FPS case, resulting in a maximum energy efficiency increase of 2.09x for MobileNetV1 on the desktop CPU, and achieving a runtime FPS of 24.39 at the cost of $0.09 \mathrm{mAP}$ reduction, in MAV-VID dataset.

Considering Fig. 4.28, in comparison to the random baseline, the inattentional policy 
predominates all over the tests, providing a higher mAP for every percentage of inattentional frames executed. Furthermore, the inattentional policy shows lower mAP degradation even at an extreme percentage of inattentional frames, suggesting that P.III method is superior at capturing the temporal dynamics inherent to videos. The highest mAP distance to the random baseline has been provided by the MobileNetV1-ConvLSTM-SSDLite in the Imagenet VID 2015 dataset, with a distance of 0.4 in mAP at $92 \%$ of inattentional frames executed. Another emergent property is that, for the case of MobileNetV1 in Imagenet VID 2015 dataset, the inattentional policy is able to match the original mAP even when executing a $40 \%$ of inattentional frames, which suggests there is a redundancy of information that does not add value to the final accuracy. In addition, thanks to our novel reward-conditional training scheme, the policy can be conditioned at inference time, providing promising results in a wide variety of sequences with varying backgrounds, changes in illumination, object size, etc. and allowing for real-time performance modulation in the context of the required application (see provided vided ${ }^{14}$ ).

Finally, at the cost of $2.2 \mathrm{M}$ parameters, a learned inattentional policy can provide adaptiveness to the video dynamics, as shown in Fig. 4.29. In this figure, two complexes and two simple scenarios, in terms of detection difficultness, are illustrated. In the complex scenarios, where the object is moving fast within the image plane, or there are ambient conditions that difficult detection, the inattentional policy performs a higher rate of full keyframes in order to maintain the detection accuracy of the object through the sequence. Nevertheless, when the scenarios are simpler, such as the case an object with slow motion in the image plane, the inattentional policy tends to neglect context to speed up computation, without missing accuracy. This adaptive behavior can be very promising in a wide variety of video object detection applications.

These results, included in P.III, have demonstrated that the correlation among frames in video sequences allowed for exploitation in order to gain efficiency in both computation latency and energy employed. Also, the recurrent network utilized in P.III, which has been learned in an end-to-end trend, enabled the successful aggregation of temporal information. In comparison to P.II, publication P.III have taken a further step in the object detection field for robotics, by taking advantage of novel video-based techniques. Additionally, P.III opened up a novel field, where DRL agents are capable of learning based on a whole distribution of reward functions in order to provide a distribution of conditioned behaviors at inference time.

\footnotetext{
${ }^{14}$ https : //vimeo.com/426725929
} 


\section{Chapter}

\section{Conclusions and Future Work}

This thesis by compendium proposed novel ML-based methods for multirotor motion control and vision-based object detection in the context of fully autonomous aerial robotic applications. The presented methods for motion control have been mainly based on DRL techniques, which have provided an end-to-end pipeline for training robotic systems in a simulated environment in order to be deployed in real-world relevant environments. The presented techniques for object detection have been mainly based on DL methods, which have validated fully synthetic and adaptive video-based strategies for embedded systems. Moreover, this doctoral work presented applications and methods that go beyond the state of the art, by performing the validation of all the proposed methods in real systems under realistic environmental conditions.

In Chapter 1, the main objective for this thesis was presented, to explore and validate $D L$ and DRL related methods in the context of aerial robotics, in order to solve novel and challenging applications, such as multirotor landing on a moving platform, non-cooperative multirotor following and video object detection in embedded systems; and with the ultimate goal of performing the experimentation in a real and relevant environment. Publications P.I - P.III shared this thematic unity and presented a clear methodical progression of the proposed techniques and results to achieve the overall goal of this thesis. In publication P.I, the foundations of DRL-based methods for multirotor motion control were laid by providing a solution for autonomous multirotor landing on top of a moving platform. In addition, P.I provided the basis for a complete framework and a vision-based landing on top of a moving platform, further developed in Rodriguez-Ramos et al. (2018). Publication P.II extended previous problem formulation to a high-dimensional and image-based state space in the context of the challenging multirotor following application. The strategy was completely approached from a synthetic learning perspective. Also, a DL-based object detection method with synthetic images from a game-engine was proposed. Publication P.III improved the previous object detection technique by including the temporal information commonly present in frames from video sequences. In addition, a novel contribution to the reinforcement learning field was proposed, where an agent is able to be trained on a distribution of reward functions to provide a conditioned behavior at inference time.

As detailed in Sections 4.2 - 4.5, all of the proposed objectives O.1 - O.6 (outlined in Section 1.3 were addressed in publications P.I - P.III. The main contributions C.1 - 
C.7 are provided in Section 1.4 and can be found in the appended publications as follows: Contributions C.1 - C.4 were provided in publications P.I - P.II, whilst contributions C.5 - C.6 were presented in P.III and contribution C.7 has been established in P.I and P.III. Contributions C.1 - C.7 are emphasized as the main contributions of this dissertation, in which DL and DRL based methods are developed to solve real-world applications providing successful and competitive results.

The proposed motion control system approaches (in publications P.I - P.II) have been rigorously verified and validated in experimental real-world scenarios. The results obtained, shown in Chapter 4, demonstrate that a pipeline where robots are able to learn in a simulated environment in order to be deployed in the real world, is feasible and can serve as the base for scaling the development of robotic applications. Indeed, robots in a simulation can safely learn end-to-end behaviors, share the increasing knowledge acquired between a high-amount of robotic instances and modulate simulated time pace (e.g. simulating weeks in real-world seconds), among others, providing a scalable robotic paradigm which can contribute to speeding up worldwide robotic development. Furthermore, regarding object detection approaches (in publications P.II and P.III), the results obtained, shown also in Chapter 4, validate the proposed techniques for object detection, in the context of both synthetic single-image and adaptive video object detection strategies. Synthetic data for object detection has been demonstrated to provide outstanding results for applications where data is scarce or anomalous examples are required. Also, the proposed video object detection techniques have validated the necessity of video-based techniques in order to increase efficiency when deploying on embedded systems for robotics. Learning techniques, and in particular DL and DRL techniques, have been demonstrated to be exceptionally versatile and have been able to solve and/or improve a wide variety of applications at different levels.

According to the TRL definitions, presented in Table 3.1, a TRL-4 in software is achieved by demonstrating the performance of a system in representative experiments under laboratory conditions. The developed technologies have gone beyond the state of the art, and are mostly in a research stage. However, they have been validated under challenging requirements and constraints, providing relevant industrial results and insights. Additionally, a TRL-6 in software and hardware is achieved in the industrial and international competition environments for Appendices $\mathrm{A}$ and $\mathrm{B}$.

On this basis, the developed technologies in the present doctoral thesis, though they notably contributed to the field, resulted from research methods. Consequently, the algorithms and results correspond mostly to prototyping stages. They have been rigorously validated in realistic conditions and are part of a constantly-growing research field, which is simultaneously feeding the industry with novel techniques to increase processes automation, efficiency, etc. On the one hand, reinforcement learning methods for motion control systems have shown promising results. Nevertheless, more realistic models or simulators are required to perform more complex or athletic maneuvers with on-board sensors. In this regard, the exploration of precise robotic models or the generation of learning-based models with real data, for the latter usage in simulated environments, compose a near-future research line of the present dissertation. Also, the exploration of generative models (e.g. GANs, autoencoders, etc.) for the production of synthetic data for low-level or imagebased realistic simulators poses a further step to the presented techniques. The proposed method for training diverse reinforcement learning agents on a distribution of reward functions to encapsulate several conditioned behaviors in one unique policy is required to be extended, assessed, and further validated for a wide variety of applications and scenarios. On the other hand, regarding DL methods for object detection, other techniques, such 
as the interleaving of various detector architectures for efficient video object detection, or semantic video segmentation strategies can be incorporated into the proposed approaches. Additionally, several techniques for computational efficiency can be included, such as neural networks half-precision, or quantization strategies, as well as the exploration of Spiking Neural Networks (SNNs).

Therefore, the results obtained in this thesis and the ongoing research and development efforts, represent a significant step towards the ultimate goal of designing and deploying fully autonomous aerial robots, capable of providing complex behaviors in challenging applications and being able to exploit learning techniques, which in turn allow for the optimization of resources and the execution of adaptive actions. 


\section{Publications}





\section{Publication I}

(C) Springer Nature B.V. 2018. Reprinted, with permission, from:

Rodriguez-Ramos, A., Sampedro, C., Bavle, H., De La Puente, P., and Campoy, P. (2018). A deep reinforcement learning strategy for UAV autonomous landing on a moving platform. Journal of Intelligent \& Robotic Systems, 93(1-2), 351-366.

doi:10.1007/s10846-018-0891-8. JCR (2019): 2.890, Manufacturing Engineering (Q1), Artificial Intelligence (Q2). 



\title{
A Deep Reinforcement Learning Strategy for UAV Autonomous Landing on a Moving Platform
}

\author{
Alejandro Rodriguez-Ramos ${ }^{1}$ (1) $\cdot$ Carlos Sampedro $^{1} \cdot$ Hriday Bavle $^{1} \cdot$ Paloma de la Puente $^{1} \cdot$ Pascual Campoy $^{1}$
}

Received: 29 September 2017 / Accepted: 18 June 2018 / Published online: 3 July 2018

(C) Springer Nature B.V. 2018

\begin{abstract}
The use of multi-rotor UAVs in industrial and civil applications has been extensively encouraged by the rapid innovation in all the technologies involved. In particular, deep learning techniques for motion control have recently taken a major qualitative step, since the successful application of Deep Q-Learning to the continuous action domain in Atari-like games. Based on these ideas, Deep Deterministic Policy Gradients (DDPG) algorithm was able to provide outstanding results with continuous state and action domains, which are a requirement in most of the robotics-related tasks. In this context, the research community is lacking the integration of realistic simulation systems with the reinforcement learning paradigm, enabling the application of deep reinforcement learning algorithms to the robotics field. In this paper, a versatile Gazebobased reinforcement learning framework has been designed and validated with a continuous UAV landing task. The UAV landing maneuver on a moving platform has been solved by means of the novel DDPG algorithm, which has been integrated in our reinforcement learning framework. Several experiments have been performed in a wide variety of conditions for both simulated and real flights, demonstrating the generality of the approach. As an indirect result, a powerful work flow for robotics has been validated, where robots can learn in simulation and perform properly in real operation environments. To the best of the authors knowledge, this is the first work that addresses the continuous UAV landing maneuver on a moving platform by means of a state-of-the-art deep reinforcement learning algorithm, trained in simulation and tested in real flights.
\end{abstract}

Keywords Deep reinforcement learning $\cdot \mathrm{UAV} \cdot$ Autonomous landing · Continuous control

\section{Introduction}

In recent years, considerable research has been conducted regarding the design, development, and operation of autonomous Unmanned Aerial Vehicles (UAVs). Multi-rotor UAVs are potentially useful in a wide variety of scenarios, from natural disasters (conflagrations, earthquakes, etc.) to automation in a broad range of industries (energy, manufacture, construction, etc.). Nevertheless, these fields of application impose enormous constraints for normal operation tasks such as taking-off, navigation, object detection, environment interaction or landing. Thus, due to their level of complexity, researchers have approached these tasks separately in a diverse

Alejandro Rodriguez-Ramos

alejandro.rramos@upm.es

1 Computer Vision and Aerial Robotics (CVAR),

Centre for Automation and Robotics (CAR),

Universidad Politécnica de Madrid (UPM), Madrid, Spain set of research lines [18, 23, 24, 35, 38] and international competitions [12, 34], e.g. International Micro-Air Vehicles competition (IMAV). ${ }^{1}$

In this context, for the last decade, the research community has focused on providing multi-rotor UAVs with the required level of autonomy for every of the previously stated tasks, from navigation in unstructured environments [7] to landing on moving platforms. In particular, the landing maneuver plays a significant role for long-term operations due to the UAV limitation of rapid battery discharge [33]. Moreover, in multi-robot operations, such as in combination with Unmanned Ground Robots (UGVs), landing and/or target following become necessary [9]. Indeed, these facts can be limiting for providing multi-rotor UAVs with the required level of autonomy in long-term missions. Due to this reason, the landing maneuver on a moving platform has been on the focus of several research lines for years $[3,5,6,14,15,20,42,46]$.

\footnotetext{
${ }^{1}$ https://imavs.org
} 
Traditionally, the landing maneuver on a moving platform has been approached by means of a wide variety of techniques, which are able to solve the problem in an analytic manner and to perform properly in some specific conditions. Most of these strategies are mainly based on perception and relative pose estimation [5, 6, 42], as well as trajectory optimization and control $[1,15,20,29,46]$.

Nevertheless, classical techniques have their limitations, in terms of model design, non-linearities approximation, disturbances rejection and efficiency of computation. In this context, machine learning techniques have proven to increasingly overcome most of these limitations, having generated high expectations in the research community since 1971, when Ivakhnenko [19] trained a 8-layer neural network using the Group Method of Data Handling (GMDH) algorithm. Nowadays, machine learning has evolved to more complex techniques, such as deep learning strategies which are capable of generalizing from large datasets of raw data information. Deep learning has opened up important research and application fields in the context of unsupervised feature extraction, where Convolutional Neural Networks (CNNs) were able to provide outstanding results in comparison to traditional computer vision techniques [26].

In the context of machine learning (and reinforcement learning) for continuous control, there are uprising problems to cope with, such as divergence of learning, temporal correlation of data, data efficiency or continuous nature of inputs and outputs. These issues have been limiting machine learning and reinforcement learning strategies for continuous control over the last years. However, recent advances in the reinforcement learning field, such as DeepMind Technologies Deep Q-Network (DQN) [31], have unveiled a new set of possibilities to solve complex human-level problems by means of novel deep reinforcement learning strategies. The key advances of DQN were the inclusion of an experience replay buffer (to overcome data correlation), and a different approach for the target Q-Network, whose weights change with the update of the main Q-Network in order to break the correlation between both networks (in contrast with the targets used for traditional supervised learning, which are fixed before learning begins) [31]. The state of the DQN algorithm is the raw image and it has been widely tested with Atari games. DQN established the base for a novel line of deep reinforcement learning solutions, but it was not designed for continuous states, which are deeply related to robotic control problems.

Based on the key improvements of DQN and the actorcritic paradigm established by Richard S. Sutton and Andrew G. Barto in their renowned reinforcement learning book [43], Lillicrap et al. proposed Deep Deterministic Policy Gradients (DDPG) [28] as an algorithm to solve continuous control problems by integrating neural networks in the reinforcement learning paradigm. DDPG is able to perform remarkably well with low dimensional continuous states and actions, but is also capable of learning from raw pixels [28].

In this work, the novel deep reinforcement learning algorithm (DDPG) has been utilised to solve a complex high level task, such as UAV autonomous landing on a moving platform. This task has been solved in simulation and real flights by means of a Gazebo-based reinforcement learning framework. The training phase has been carried out in Gazebo ${ }^{2}$ [48] and RotorS simulator [13], which provide realistic simulations that are helpful for a quick transition to real flight scenarios. The testing phase has been performed in both simulated and real flights.

\subsection{Related Work}

The problem of UAV autonomous landing on both static and moving platforms is of utmost importance for real world applications [9, 33]. Given the complexity of the challenge, a number of previous works focus mostly on specific solutions for components such as perception and relative pose estimation $[5,6,42]$ or trajectory optimization and control $[1,15,20,29,46]$. Other research lines explore coupled methods mostly related to Image-Based Visual Servoing (IBVS) [27] and, in this direction, novel advanced algorithms which also incorporate constant force disturbance estimation have been proposed [39].

Regarding the control maneuvers when the relative state of the vehicles is assumed to be known, popular techniques include different kinds of guidance and rendezvous laws $[15,20]$ which sometimes are augmented with velocity controllers for a faster approaching phase [3]. When a desired meeting point is obtained, incorporating feedforward inputs allows for a faster response against track following errors [29] and the determination of optimal rendezvous trajectories can also take wind disturbances into account [1]. PID controllers are the preferred option for aggressive landing from relatively short distances [3, 5, 47], while an adaptive control schema presents enhanced robustness [18, 24]. A discrete-time non-linear model predictive controller which optimizes both the trajectories and the landing time was developed to address the difficult problem of landing on top of moving inclined platforms [46].

Even if only tested on static platform landing tasks, innovative bio-inspired strategies have proven to perform well in the real world, employing a time-to-contact (TTC) indicator [22]. Intelligent control and machine learning based methods are very promising too, since they provide the ability

\footnotetext{
$\overline{{ }^{2} \mathrm{http}: / / \text { gazebosim.org }}$
} 
to deal with different system dynamics in different environments and landing circumstances [4]. Recent contributions have proposed neural network backpropagation controllers [4] for landing on top of a static platform and classical discrete reinforcement learning approaches have also been used in the literature, such as the approach proposed by Shaker et al. [40], where an LSPI algorithm was used to land on top of a static platform. Both state and actions were part of a discrete space and the main sensor to estimate the state was a camera. The UAV was able to perform a landing maneuver on a static platform in a simulated environment.

The previously mentioned novel reinforcement learning methodologies are strongly related to deep learning strategies, since their theory is intrinsically linked. Concerning deep learning for UAV indoor navigation tasks, recent advances have driven to a successful application of CNNs in order to map images to high-level behaviour directives (e.g. turn left, turn right, rotate left, rotate right) [23, 35]. In [35], the Q function is estimated through a $\mathrm{CNN}$, which is trained in simulation and successfully tested in real experiments. In [23], discrete actions are directly mapped from raw images. In all stated methods, the learned model is run offboard, usually taking advantage of a GPU in an external laptop.

In [16], a Deep Neural Network (DNN) model was trained to map image to action probabilities (turn left, go straight or turn right) with a final softmax layer, and tested onboard by means of an Odroid-U3 processor. The performance is later compared to two automated methods (SVM and a method in [38]) and two human observers.

On the other hand, deep learning for low-level motion control is challenging, since dealing with continuous and multi-variable action spaces can become an intractable problem. Nevertheless, some recent advances have proposed novel methods to learn low-level control policies from imperfect sensor data in simulation [21, 49]. In [49], a Model Predictive Controller (MPC) was used to generate data at training time in order to train a DNN policy, which was allowed to access only raw observations from the UAV onboard sensors. In testing time, the UAV was able to follow an obstacle-free trajectory even in unknown situations. In [21], the well-known Inception v3 model (pre-trained CNN) was adapted in order to enable the final layer to provide six action nodes (three transitions and three orientations). After re-training, the UAV managed to cross a room filled with a few obstacles in random locations.

On the side of deep reinforcement learning, some recent algorithms are able to perform slightly better than DDPG, in terms of training time and for low-dimensional continuous tasks. In [17], Normalized Advantage Functions (NAF) or continuous deep Q-learning algorithm is able to solve continuous problems in simulation, by the use of a neural network that separately outputs a value function $V(x)$ and an advantage term $A(x, u)$ [17]. This representation allows to simplify more standard actor-critic style algorithms, while preserving the benefits of non-linear value function approximation [17]. In [30], several agents (from 1 to 16) are run in parallel threads, enabling the possibility of stable training of neural networks with both value-based and policy-based methods, off-policy as well as on-policy methods, and in discrete as well as continuous domains. Also, Asynchronous Advantage Actor-Critic (A3C) shows that stable online Q-learning is possible without experience replay [30]. Both [17] and [30] have been tested in simulated environments, such as MuJoCo [44] and/or TORCs [11].

Finally, concerning the framework for training and testing novel deep reinforcement learning algorithms for robotics, recent developments point to extend the OpenAI $\mathrm{Gym}^{3}$ reinforcement learning training/test bench to a widely-used robotics simulator, such as Gazebo simulator. In [48], a complete open source test bench is released, with simulation frequency up to real time and meant for an specific model of UAV and UGV.

\subsection{Contributions}

Our proposed method differs from previous work in the following aspects: (i) A Gazebo-based reinforcement learning framework has been established. This framework is versatile-enough to be adapted to other types of algorithms, environments and robots. (ii) A novel deep reinforcement learning algorithm (DDPG) has been adapted and integrated into our Gazebo-based simulation framework. (iii) The landing maneuver on a moving platform has been solved by means of DDPG algorithm, in both simulated and real flights.

Please note that we address the full problem, with continuous state and actions spaces. Also, as an indirect result, we have demonstrated the feasibility of a powerful work flow, where robots can be trained in simulation and tested in real operation environments. To the best of the authors knowledge, this is the first work that addresses the UAV landing maneuver on top of a moving platform by means of a state-of-art deep reinforcement learning algorithm, trained in simulation and tested in real flights.

The remainder of the paper is organized as follows: Section 2 presents a brief introduction on the reinforcement learning theory and a short explanation on the basics of DDPG algorithm. Section 3 details the presentation and description of our Gazebo-based reinforcement learning framework and the design of the experiment which meets all the constraints required in the deep reinforcement learning paradigm for autonomous UAV landing on a moving

\footnotetext{
${ }^{3}$ Open test bench for reinforcement learning algorithms: https://gym. openai.com
} 
platform. Section 4 presents the simulated and real-flight experiment results. Finally, Section 5 provides conclusions and future work optimizations and research lines.

\section{Background}

In reinforcement learning, an agent is defined to interact with an environment, seeking to find the best action for each state at any time step. The agent must balance exploration and exploitation of the state space in order to find the optimal policy which maximizes the accumulated reward from the interaction with the environment. In this context, an agent modifies its behaviour or policy with the awareness of the states, actions taken and rewards for every time step. Indeed, reinforcement learning involves an optimization process throughout the whole state space, in order to maximize the accumulated reward. Robotic problems are often task-based with temporal structure. These type of problems are suitable to be solved by means of a reinforcement learning framework [25].

The standard reinforcement learning theory states that an agent is able to obtain a policy, which maps every state $s \in \mathbb{S}$ to an action $a \in \mathbb{A}$, where $\mathbb{S}$ is the state space (possible states of the agent in the environment) and $\mathbb{A}$ is the finite action space. The inner dynamics of the agent are represented by the transition probability model $p\left(s_{t+1} \mid s_{t}, a_{t}\right)$ at time $t$. The policy can be stochastic $\pi(a \mid s)$, with a probability associated to each possible action, or deterministic $\pi(s)$. In each time step, the policy determines the action to be chosen and the reward $r\left(s_{t}, a_{t}\right)$ is observed from the environment. The goal of the agent is to maximize the accumulated discounted reward $R_{t}=\sum_{i=t}^{T} \gamma^{i-t} r\left(s_{i}, a_{i}\right)$ from a state at time $t$ to time $T$ ( $T=\infty$ for infinite horizon problems) [43]. The discount factor $\gamma$ is defined to allocate different weights for the future rewards.

For a specific policy $\pi$, the value function $V^{\pi}$ in Eq. 1 is a representation of the expectation of the accumulated discounted reward $R_{t}$ for each state $s \in \mathbb{S}$ (assuming a deterministic policy $\pi\left(s_{t}\right)$ ).

$V^{\pi}\left(s_{t}\right)=\mathbb{E}\left[R_{t} \mid s_{t}, a_{t}=\pi\left(s_{t}\right)\right]$

An equivalent of the value function is represented by the action-value function $Q^{\pi}$ in Eq. 2 for every action-state pair $\left(s_{t}, a_{t}\right)$.

$Q^{\pi}\left(s_{t}, a_{t}\right)=r\left(s_{t}, a_{t}\right)+\gamma \sum_{s_{t+1}} p\left(s_{t+1} \mid s_{t}, a_{t}\right) V^{\pi}\left(s_{t+1}\right)$

The optimal policy $\pi^{*}$ shall be the one which maximizes the value function (or equivalently the action-value function), as in Eq. 3.

$\pi^{*}=\underset{\pi}{\arg \max } V^{\pi}\left(s_{t}\right)=\underset{a_{t}}{\arg \max } Q^{*}\left(s_{t}, a_{t}\right)$
A general problem in real robotic applications is that the state and action spaces are often continuous spaces. A continuous state and/or action space can make the optimization problem intractable, due to the overwhelming set of different states and/or actions. Reinforcement learning methods, as a general framework for representation, are enhanced through deep learning to aid the design for feature representation, which is known as deep reinforcement learning.

In the context of state-of-the-art deep reinforcement learning algorithms, DDPG represents one successful application of neural networks to the reinforcement learning paradigm, and it is able to solve continuous control problems. As previously stated, DDPG [28] is a policybased deep reinforcement learning algorithm designed to work with both continuous state and actions spaces. Policy-based reinforcement learning methods aim towards directly searching the optimal policy $\pi^{*}$, which provides a feasible framework for continuous control. If the target policy $\pi^{*}$ is a deterministic policy $\mu$, the $\mathrm{Q}$ function (see Eq. 4) can be learned off-policy, using transitions (from an environment $E$ ) which are generated from a different stochastic behaviour policy $\beta$ [28].

$Q\left(s_{t}, a_{t}\right)=\mathbb{E}_{r_{t}, s_{t+1} \sim E}\left[r\left(s_{t}, a_{t}\right)+\gamma Q^{\mu}\left(s_{t+1}, \mu\left(s_{t+1}\right)\right)\right]$

A function approximator, parametrized by $\theta^{Q}$, is considered in DDPG to approximate the $\mathrm{Q}$ function. It is optimized by minimizing the loss $L\left(\theta^{Q}\right)$ of Eq. 5 .

$L\left(\theta^{Q}\right)=\mathbb{E}_{s_{t} \sim \rho^{\beta}, a_{t} \sim \beta, r_{t} \sim E}\left[\left(Q\left(s_{t}, a_{t} \mid \theta^{Q}\right)-y_{t}\right)^{2}\right]$

where

$y_{t}=r\left(s_{t}, a_{t}\right)+\gamma Q\left(s_{t+1}, \mu\left(s_{t+1}\right) \mid \theta^{Q}\right)$

The key changes for this large non-linear approximators to converge (in discrete spaces) were: the use of a replay buffer, and a separate target network for calculating $y_{t}$, as firstly proven by DQN [31]. In order to deal with large continuous state and action spaces, DDPG adapted the actor-critic paradigm introduced in [41], with two neural networks to approximate a greedy deterministic policy (actor) and the $Q$ function (critic). DDPG method learns with an average factor of 20 times fewer experiences steps than DQN [28].

The actor network is updated by following and applying the chain rule to the expected return from the start distribution $J$ with respect to the actor parameters (see Eq. 7).

$\nabla_{\theta \mu} J \approx \mathbb{E}_{s_{t} \sim \rho^{\beta}}\left[\left.\nabla_{\theta \mu} Q\left(s, a \mid \theta^{Q}\right)\right|_{s=s_{t}, a=\mu\left(s_{t} \mid \theta^{\mu}\right)}\right]$

An advantage of the off-policy methods is that exploration can be treated independently from learning. In this case, exploration is carried out throughout autocorrelated Ornstein-Uhlenbeck exploration noise [45]. 


\section{Proposed Approach}

In this section, a complete explanation of our Gazebo-based reinforcement learning framework is included. Furthermore, the formulation of the UAV landing on a moving platform problem, within the reinforcement learning paradigm, is detailed.

\subsection{Reinforcement Learning Simulation Framework}

Traditionally, reinforcement learning algorithms have been tested and validated by means of generic tools for simulation (e.g. Matlab, Simulink). The performance of other classic reinforcement learning algorithms was not powerful enough to overcome high-level and complex problems, neither in the context of continuous states and actions spaces, or working with continuous physics in realistic simulators (e.g. Gazebo simulator). Nevertheless, since there has been an increasing improvement in the performance of this kind of algorithms, simulation systems have been following this complexity trend, allowing a feasible and stable training of reinforcement learning agents under realistic and continuous simulators.

OpenAI Gym is an open-source toolkit for developing and comparing reinforcement learning algorithms [8]. Currently, most of the available environments for testing in OpenAI Gym are 2D or Atari-like games, with a diverse range of complexity examples, but lacking more realistic environments for robotic simulation. In robotics, this type of training/testing environments are not directly available for the research community.

On the other hand, the Gazebo simulator is often a prime example of a versatile and realistic simulation system for robotics, normally aided by the well-known Robot Operating System (ROS) middleware [32]. Hence, taking advantage of the versatility of this broadly-used simulation system, we have designed and implemented a Gazebobased reinforcement learning framework. The aim of this framework is to provide a predefined interface between the reinforcement learning algorithm, the environment interface and the Gazebo simulator (or other type of simulators). Also, it has been designed in such a way that it can be extended in the future to different types of reinforcement learning algorithms, environments and robots.

As previously stated, in reinforcement learning, an agent interacts with an environment and tries to maximize the accumulated reward in each time step. In our framework, the communication channel between the agent and the environment is implemented through commonly-used ROS communication tools, which provide a standardized way of communication (see Fig. 1). Also, some reinforcement learning problems require the simulation time to be deliberately iterated (the clock is paused), in order to complete computationally-expensive training steps. In this scenario, where the Gazebo simulator clock is paused, communication via ROS tools is not possible, since ROS uses Gazebo clock to forward its messages through the network. In order to overcome these issues, an additional shared memory communication channel has been implemented, which can be used in case the Gazebo clock is paused and the simulation is being actively iterated. This functionality provides the developer with full control on the simulation time, allowing the interaction of a Gazebo plugin with Gazebo simulator and the shared memory interface, which can be a requirement in several real world problems.

Furthermore, in our framework, the agent component which represents the classical reinforcement learning agent, is capable of receiving experience vectors and rewards from the environment in order to find the optimum action to be taken in each time step. Our implementation of the agent is also capable of logging representative data which can be used to determine whether it is actually learning or whether the algorithm has otherwise diverged. On the other hand, the environment interface shown in Fig. 1 represents a partial implementation of the classical reinforcement learning environment, since in this case, Gazebo simulator and Aerostack architecture [37] constitute an important part of the whole reinforcement learning environment. Aerostack is a system architecture and open-source multipurpose software framework for autonomous multi-UAV

Fig. 1 Diagram of our reinforcement learning simulation framework. The theoretical concept of the reinforcement learning environment is highlighted in pale red. Simulation systems or multi-purpose architectures are highlighted in pale green

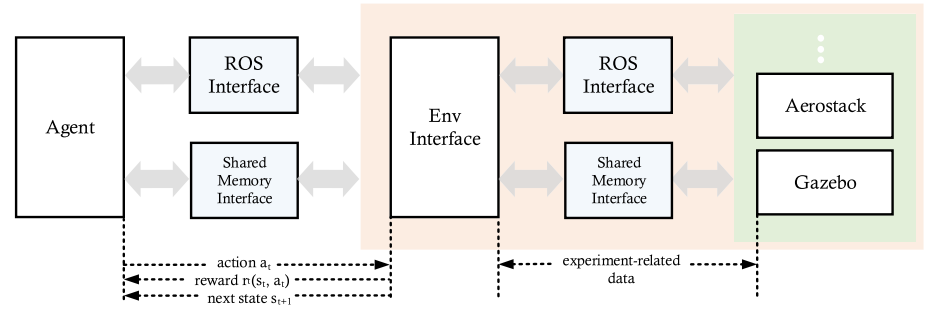


operation. It has been used to enable the operation of the UAVs in both training and testing time, though its full explanation is out of the scope of this work. For further information, please refer to [37].

In this framework, the environment interface shown in Fig. 1 implements an interface between Gazebo/Aerostack and the agent, being in charge of parsing all the incoming data, in order to adapt it to an intelligible structure which the agent can use. Furthermore, taking into consideration future extensions of either agents, environments, robots or simulation systems, the framework has been designed in a versatile manner at a programming level. Since all the communication interfaces are standard and cross-language, both the agent and the environment interface can be implemented in a wide variety of programming languages, such as $\mathrm{C}++$, Python, or Java.

Finally, our framework is designed to be used with Gazebo, but it can be adapted to any other simulation systems (as well as simulated robots), due to the standard nature of its communications. Also, the simulation time can be speeded up or slowed down, in order to reduce training times and to adapt the simulation to computationallyexpensive experiments, respectively.

\subsection{Reinforcement Learning Based Formulation}

In the context of reinforcement learning, the formulation of the experiment can be decisive for the algorithm to converge, since there are an increasing number of possible designs which ideally would lead to the same result. In practice, the formulation of the state and action spaces, as well as the design of the reward function, determines the speed of convergence and even the possibility of divergence of the reinforcement learning algorithm. We have designed the state, action and reward function in a way that it minimizes information passed to the agent, speeds up learning and avoids learning divergence.

As previously stated, a reinforcement learning experiment is defined by the state space $s \in \mathbb{S}$, the action space $a \in \mathbb{A}$ and the reward function $r$. In our proposed approach, the state space $\mathbb{S}$ is defined by Eq. 8 .

$\mathbb{S}=\left\{p_{x}, p_{y}, p_{z}, v_{x}, v_{y}, C\right\}$

Where $p_{x}, p_{y}$ and $p_{z}$ are the positions of the UAV with respect to the Moving Platform (MP) in $x, y$ and $z$ axes respectively at time $t, v_{x}$ and $v_{y}$ are the velocities of the UAV with respect to the MP in $x$ and $y$ axes respectively at time $t$ and $C$ is the binary state of a pressure sensor located on the top of the horizontal surface of the MP. All the sensory information is retrieved from Gazebo simulator and parsed by the environment interface component, as shown in Fig. 2. Regarding the action space $\mathbb{A}$, it is defined by Eq. 9 .

$\mathbb{A}=\left\{a_{x}, a_{y}\right\}$

Where $a_{x}$ and $a_{y}$ are the reference velocities, input to the velocity controller (see Fig. 2), in $x$ and $y$ axes at time $t$. In this paper, the velocity reference in the $\mathrm{z}$ axis has not been included in the action space. This is due to the fact that we are tackling a complex problem with continuous state and action spaces and the full behaviour is completely self-learned in simulation, by means of a deep reinforcement learning algorithm not previously tested on this type of robotic tasks. Hence, the inclusion of $\mathrm{z}$ axis has been left as future work since it involves a much higher order of complexity out of the scope of this study. Instead, a constant velocity reference is commanded in the $\mathrm{z}$ axis in each time step. This fact simplifies the action space, increasing the speed of convergence of the algorithm without losing generality of the approach. The resulting state and action spaces are a continuous 6-dimensional space and a continuous 2-dimensional space respectively, with normalized variables ranging from +1 to -1 values.

The reward function is one of the most important components in the reinforcement learning framework. A proper design of the reward function can lead to a faster convergence of the algorithm and a better performance at testing time. In our proposed approach, where the agent is meant to generate continuous control actions, the reward

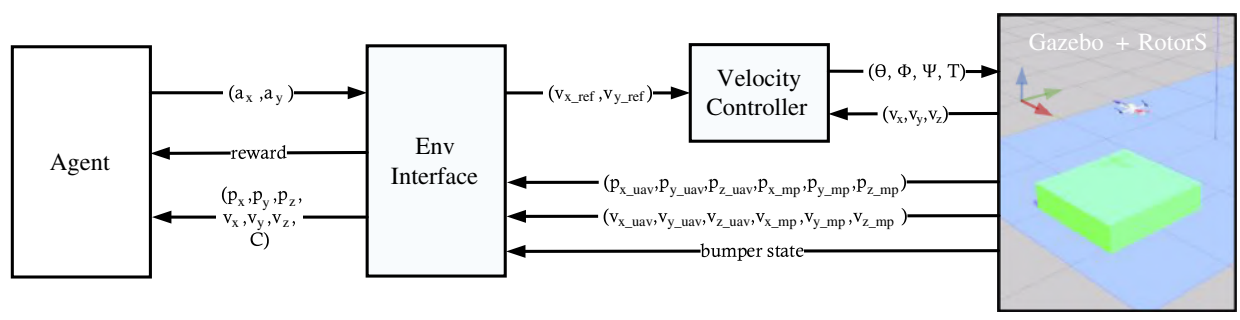

Fig. 2 Architecture of our proposed reinforcement learning framework for the case of the experiment of study 
function shall be designed in such a way that it rewards smooth actions with respect to time. The resulting reward function $r$ is defined by Eqs. 10 and 11 .

$$
\begin{aligned}
\text { shaping }_{t}= & -100 \sqrt{p_{x}^{2}+p_{y}^{2}}-10 \sqrt{v_{x}^{2}+v_{y}^{2}}-\sqrt{a_{x}^{2}+a_{y}^{2}} \\
& +10 C\left(1-\left|a_{x}\right|\right)+10 C\left(1-\left|a_{y}\right|\right)
\end{aligned}
$$

$r=$ shaping $_{t}-$ shaping $_{t-1}$

As can be inferred from Eq. 10, the shaping function explicitly differentiates between the importance of minimizing the position with respect to the MP, the velocity with respect to the MP and the generated actions (each variable is weighted by a different coefficient). Following this fashion, the agent is able to coarsely learn to minimize its position with respect to the MP and to subsequently optimize its behaviour in order to generate smoother velocity references, which leads to a less aggressive movement. Also, the $C$ coefficient rewards the agent as soon as the UAV lands on the MP and the velocity references are decreased to their absolute minimum.

In addition, shaping is a popular method for speeding up reinforcement learning in general, and goal-directed exploration in particular [10]. It increases the speed of convergence of a reinforcement learning algorithm by transferring knowledge about the current progress on the task to be solved, in this case, with respect to the previous state of the agent. Nevertheless, it requires significant design effort, and results in less autonomous agents. Also, it may alter the optimal solution, leading to unexpected final behaviour. In this work, a shaping method is applied in an non-invasive trend, by informing the agent about its instantaneous progress and avoiding instability and algorithm divergence.

Also, it is assumed that both the position and velocity of the UAV and the MP are available at training time (ground truth data). Nevertheless, as stated in this section, the agent is only aware of its position and velocity with respect to the MP, enabling this approach to work also in the absence of absolute positioning systems, such as Global Navigation Satellite Systems (GNSS). It has to be noted that even if the agent has been trained with ground truth data, it is capable of performing the landing maneuver with noisy simulated and real data, as shown in Section 4.2.

The training procedure is based on an adapted implementation of stated DDPG algorithm included in our framework. In our case, the actor and critic neural networks (and their corresponding target networks) are feed-forward neural networks with two hidden layers of 200 and 100 units each. The activation function of each unit of a hidden layer is a Recified Linear Unit (ReLU). The input and output layer dimensions of the actor network are based on the state and action dimensions (6 and 2 units), respectively (see
Eqs. 8 and 9). The activation function of the output layer is a tanh function, bounded to the range of $[-1,1]$. The output layer of the critic network has one unit with a linear activation function in order to provide an estimation of the Q-function.

\section{Experiments and Results}

This section aims to provide a full explanation about the experiments designed and implemented to validate the whole training and testing pipeline. A detailed description of the training experiments in simulation, as well as the testing experiments in both simulation and real flights, is included. Results are shown and discussed, as well the hardware and software specifications which have been used to carry out the described experiments. A complete video of the whole set of training and testing experiments can be found in https://vimeo.com/235350807.

\subsection{Experimental Setup}

In this section, the proposed experimental setup for simulated and real flights is described. The agent has been implemented in Python 2.7, due to the availability of the most common machine learning libraries. In this work, the Tensorflow library [2] has been used as the main basis of the algorithm and it can run on both Central Processing Unit (CPU) and Graphical Processing Unit (GPU). The GPU involved in the training phase was a Nvidia GeForce GTX970 and in the testing phase was a Nvidia GeForce GTX950M. In the case of the environment interface, it has been implemented in $\mathrm{C}++$ (under the standard $\mathrm{C}++11$ ), in order to take advantage of the benefits of our Aerostack architecture [37]. ROS Kinetic has been used as the communication framework. The operating system utilised for running the processes involved in both simulated and real flights is Ubuntu 16.04 LTS.

\subsubsection{Simulated Training and Testing Phases}

A simulated environment has been created in Gazebo 7 for both simulated training and testing phases. A UAV model and a minimalistic model of a MP (see Fig. 3a) have been included in an adapted version of RotorS simulator [13]. RotorS simulator emulates the autopilot and all the required sensors for a UAV to perform autonomous maneuvers, such as Inertial Measurement Unit (IMU), lidar and/or cameras (RGB or RGB-D). We have selected an AsTec Hummingbird as the UAV to perform the landing maneuver in simulation. A simulated pressure sensor has been included on the surface of the MP, in order to inform the agent about whether the UAV has properly landed or not. 
Fig. 3 Simulation and real environment scenarios

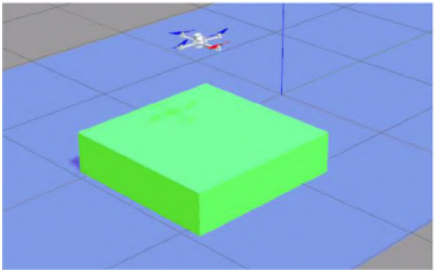

(a) UAV and MP in the training and testing simulation environment.

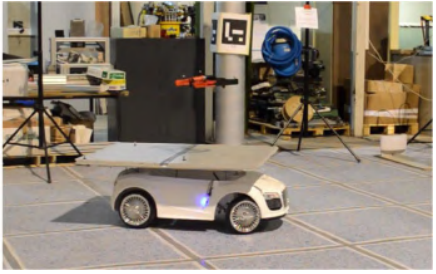

(b) UAV and MP in the testing real environment.
The environment interface, which is in charge of parsing all the incoming data, receives position and velocity ground truth data from the Gazebo simulator and sends the velocity references to the Aerostack velocity controller via ROS topics. The state and reward is sent back to the agent via ROS services. The whole simulated training and testing phases have been carried out in a real-time Gazebo simulation, with an agent frequency of $20 \mathrm{~Hz}$, an asynchronous environment interface, a velocity controller frequency of $30 \mathrm{~Hz}$ and Gazebo ground truth frequency of $100 \mathrm{~Hz}$. The main differences between simulated training and testing phases are:

1. Simulated training phase The trajectory of the MP is linear and periodic with a maximum velocity of $1 \mathrm{~m} / \mathrm{s}$. The measured position and velocity of both the UAV and the MP are ground truth data with no noise. The permitted horizontal area for the UAV to fly is a rectangle of $3 \mathrm{~m} \times 6 \mathrm{~m}$ (it has been empirically set to provide the minimum feasible area which allows to learn the landing maneuver).

2. Simulated testing phase The trajectory of the MP can be linear and periodic with a maximum velocity of $1 \mathrm{~m} / \mathrm{s}$ or non-linear and non-periodic with a maximum velocity of $1 \mathrm{~m} / \mathrm{s}$. The measured position and velocity of both the UAV and the MP are ground truth data with Gaussian noise $(\mu=0$ and $\sigma=1)$ in every variable of the agent state. The permitted horizontal area for the UAV to fly is a rectangle of $5 \mathrm{~m} \times 9 \mathrm{~m}$.

\subsubsection{Real-Flight Testing Phase}

A replica of the simulated environment has been created for the real-flight testing phase. The MP, which was designed and built for previous works on autonomous landing [34, 36], is able to move in linear periodic trajectories (with rails) and in arbitrary trajectories. The selected UAV platform was a Parrot Bebop ${ }^{4}$ due to its small size, robust

\footnotetext{
${ }^{4}$ http://global.parrot.com/mx/productos/bebop-drone/
}

control and higher flight velocity (see Fig. 3b). This UAV platform is provided with an on-board autopilot which can be commanded throughout a wireless WiFi channel. The remaining processes, such as the agent, the environment interface and the Aerostack components are run off-board. Tensorflow library calls are computed on a laptop GPU (Nvidia GTX950M). The rest of the required routines are computed on the CPU. The UAV and MP position and velocity information is provided by an OptiTrack Motion Capture system (MoCap) which covers an area of approximately $4 \mathrm{~m} \times 6 \mathrm{~m}$. The frequency of the agent is 20 $\mathrm{Hz}$, the environment interface is asynchronous, the velocity controller runs at $30 \mathrm{~Hz}$ and the motion capture system frequency is $100 \mathrm{~Hz}$. The communication with the UAV is carried out trough $\mathrm{WiFi}$ at $2.4 \mathrm{GHz}$.

\subsection{Results and Discussion}

In this work, the full landing maneuver has been trained in simulation throughout 4500 episodes (approximately $720 \mathrm{k}$ training steps over $10 \mathrm{~h}$ ). In this setup, an episode consists of a full landing trial on top of the MP and it is composed of a maximum of 900 training steps. As previously stated, the agent interacts with the environment every $0.05 \mathrm{~s}$ (at a frequency of $20 \mathrm{~Hz}$ ), which corresponds to one training step. In each training step both actor and critic network weights are being optimized by means of Adam optimizer and with a base learning rate of $10^{-4}$ and $10^{-3}$, respectively. The selected minibatch size has been 64 . In every episode, the UAV and the MP are initialized at a random position of the horizontal plane ( $x$ and $y$ axes). The experiment finishes when the UAV touches the ground or the number of training steps exceeds the maximum per episode. Following this trend, the experiment is repeated in a wide variety of conditions to provide a complete range of experiences which the agent can learn from in order to maximize its accumulated reward over time.

In Fig. 4, the moving average and standard deviation of the accumulated reward for the full simulated training and testing phase are depicted. In the training phase, an 


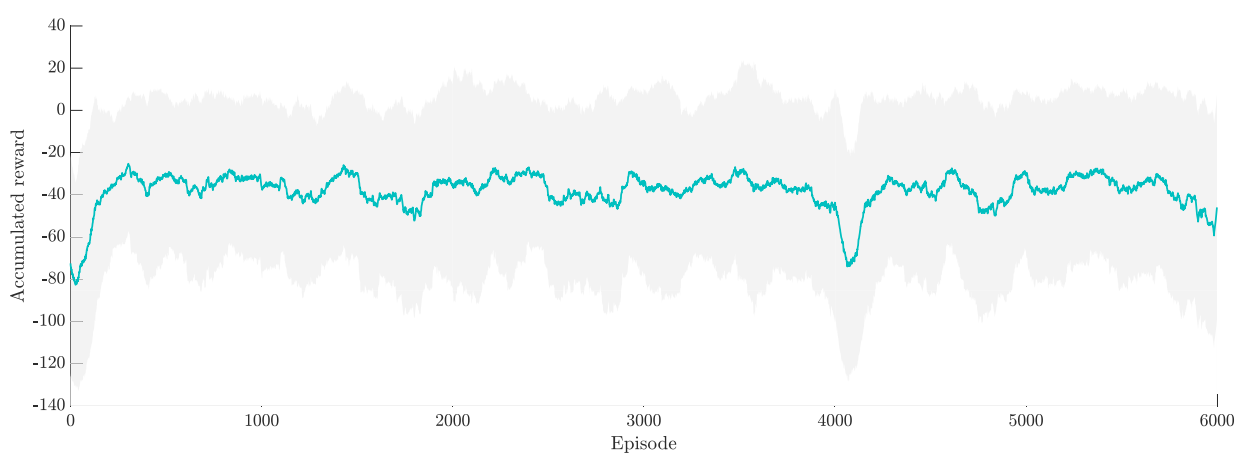

(a) Moving average and standard deviation of the accumulated reward per episode (moving window of 100 episodes) for the full simulated training phase.

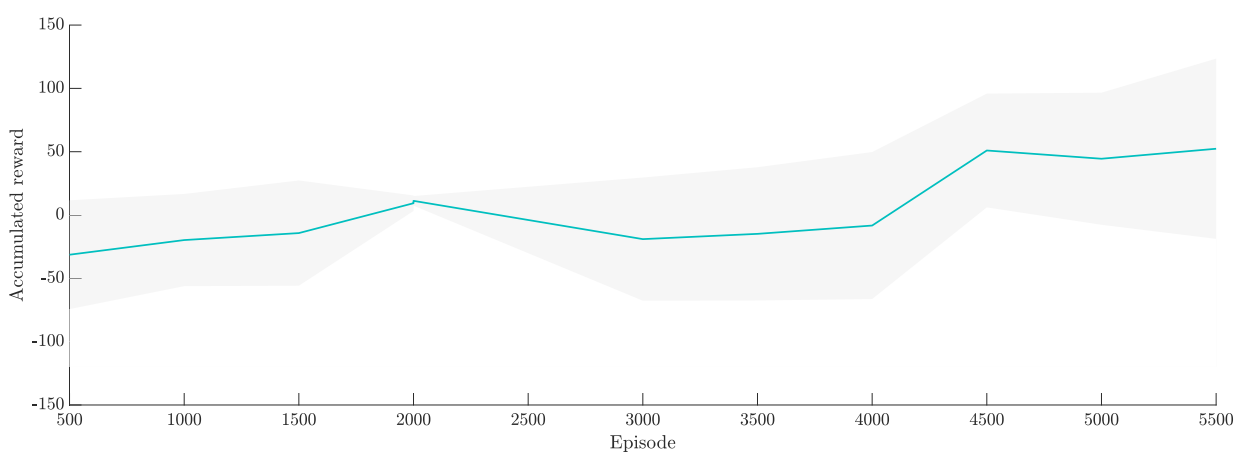

(b) Moving average and standard deviation of the accumulated reward per episode (moving window of 3 episodes) for the full simulated testing phase (within the learning process).

Fig. 4 Average and standard deviation of the accumulated reward, for both simulated training and testing phases

Ornstein-Uhlenbeck exploration noise [45] is added to the actions provided by the agent. For this reason, the evolution of the accumulated reward of the training phase is not stable (only its average is stable), as shown in Fig. 4a. Nevertheless, the evolution of the accumulated reward shows an improvement after the first 400 episodes, and subsequently a stabilization of the moving average and standard deviation for the rest of the training phase. Thus, the representation of the moving average and standard deviation of the accumulated reward in the training phase is helpful but does not show the real performance of the agent.

In Fig. $4 \mathrm{~b}$, the moving average and standard deviation of the accumulated reward, for the simulated testing phase, are shown. Every 500 episodes of the training phase, 10 episodes of the testing phase have been carried out. In a test episode, the actions provided by the agent are directly mapped (without adding noise) to the environment as a way of determining its real performance. As shown in Fig. 4b, the average accumulated reward increases until episode 4500 , where it remains approximately stable. As explained in the rest of the subsection, episode 4500 has been chosen as the first episode where the agent is fully trained, due to the stabilization of the average accumulated reward and the performance shown in the actions provided.

Every experiment included in this section corresponds to a successful landing trial on top of the MP. In Fig. 5a, part of the state and action signals of episode 500 of the simulated testing phase are depicted. The most important components of the state, in terms of representation of the performance of the agent, are the position of the UAV with respect to the MP $\left(p_{x}, p_{y}\right)$ and the velocity of the UAV with respect to the MP $\left(v_{x}, v_{y}\right)$. These components allow to 

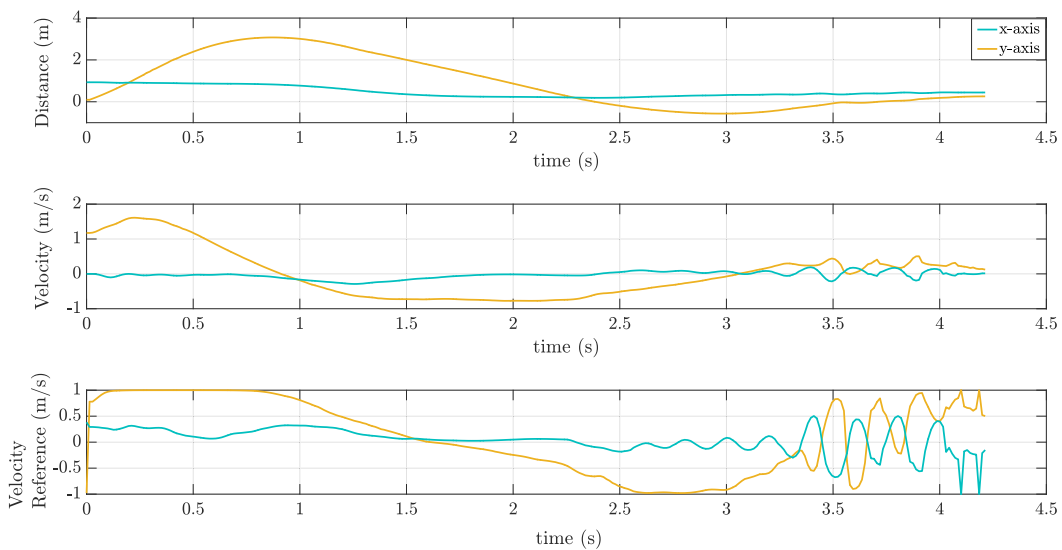

(a) Partial state and actions signals of episode 500 (test). (Top) Position of the UAV with respect to the MP, in $\mathrm{x}$ and $\mathrm{y}$ axes. The final position of the UAV with respect to the MP is $0.44 \mathrm{~m}$ and $0.42 \mathrm{~m}$, in $\mathrm{x}$ and $\mathrm{y}$ axes respectively. (Middle) Velocity of the UAV with respect to the MP, in $\mathrm{x}$ and $\mathrm{y}$ axe. (Bottom) Velocity reference commands (actions) generated by the agent, in world coordinates and in $\mathrm{x}$ and $\mathrm{y}$ axes.
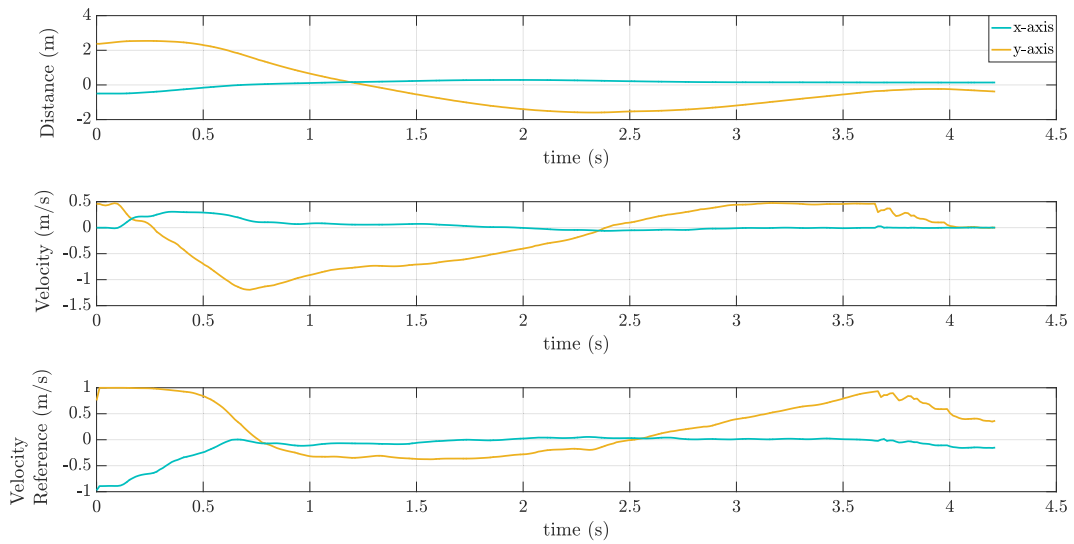

(b) Partial state and actions signals of episode 4500 (test). (Top) Position of the UAV with respect to the MP, in $\mathrm{x}$ and $\mathrm{y}$ axes. The final position of the UAV with respect to the MP is $0.14 \mathrm{~m}$ and $-0.37 \mathrm{~m}$, in $\mathrm{x}$ and $\mathrm{y}$ axes respectively. (Middle) Velocity of the UAV with respect to the MP, in $\mathrm{x}$ and $\mathrm{y}$ axes. (Bottom) Velocity reference commands (actions) generated by the agent, in world coordinates and in $\mathrm{x}$ and $\mathrm{y}$ axes.

Fig. 5 Partial state and actions signals of two test episodes (simulated testing phase) 
infer the performance in the $\mathrm{x}$ and $\mathrm{y}$ axes (parallel to the ground plane), since the performance in the $\mathrm{z}$ axis remained constant in these experiments (refer to Section 3.2). Also, the actions provided by the agent $\left(a_{x}, a_{y}\right)$ are determinant to validate the performance for a real application. As shown in Fig. 5a, the actions generated by the agent in the episode 500 are not optimum, since it keeps on generating velocity reference commands even when landed. Nevertheless, as suggested by Fig. $4 \mathrm{a}$, after episode 400 , the behaviour of the agent is close to the optimum, being able to perform a full landing maneuver in most of the testing episodes, but with oscillating control actions when touching the MP.

In Fig. 5b, both position and velocity with respect to the MP converge to approximately zero (exact zero is not practically possible either in the context of a realistic simulation or in a real flight), and in a continuous and smooth trend, which is the desired behaviour. Furthermore, the velocity reference commands generated by the agent converge approximately to zero as well. Figure $5 \mathrm{~b}$ represents the optimum performance of the agent in the absence of noise for the setup presented in this work. Note that due to the lack of friction of the simulated MP, the UAV is able to land on approximately the center of the MP, but it slightly slides from this position over time and the agent has learned to compensate this effect (see supplementary video provided in the beginning of Section 4). Nevertheless, the UAV is still on top of the MP, which is considered a successful landing.
Table 1 Mean and standard deviation metrics over 150 landing trials in two different simulated scenarios (for our selected actor network of episode 4500)

\begin{tabular}{llrc}
\hline Scenario & tland $(\mathrm{s})$ & \multicolumn{1}{l}{$\begin{array}{l}\mathrm{x}(\mathrm{m}) \\
\mathrm{y}(\mathrm{m})\end{array}$} & $\mathrm{SR}(\%)$ \\
\hline Slow & $13.5 \pm 1.56$ & $-0.01 \pm 0.38$ & 90.6 \\
& & $0.06 \pm 0.47$ & \\
Fast & $17.76 \pm 1.52$ & $0.04 \pm 0.42$ & 73.3 \\
& & $-0.11 \pm 0.49$ & \\
\hline
\end{tabular}

On the other hand, in order to test the capability of generalization and robustness of the DDPG algorithm in simulation, a test experiment with added noise has been performed. In this experiment, a Gaussian random variable ( $\mu=0$ and $\sigma=1$ ) has been added to every component of the agent state, resulting in the plots of Fig. 6. As shown, both the position and velocity of the UAV with respect to the MP are signals with a high level of gaussian noise, but the agent is still able to perform a proper landing maneuver (the position of the UAV with respect to the MP still converges to the origin). The velocity reference commands generated by the agent are notably noisy, which can be problematic in some other velocity control strategies (it may lead to over oscillation). However, in the context of a linear velocity controller, the final behaviour is more erratic but does not become unstable.
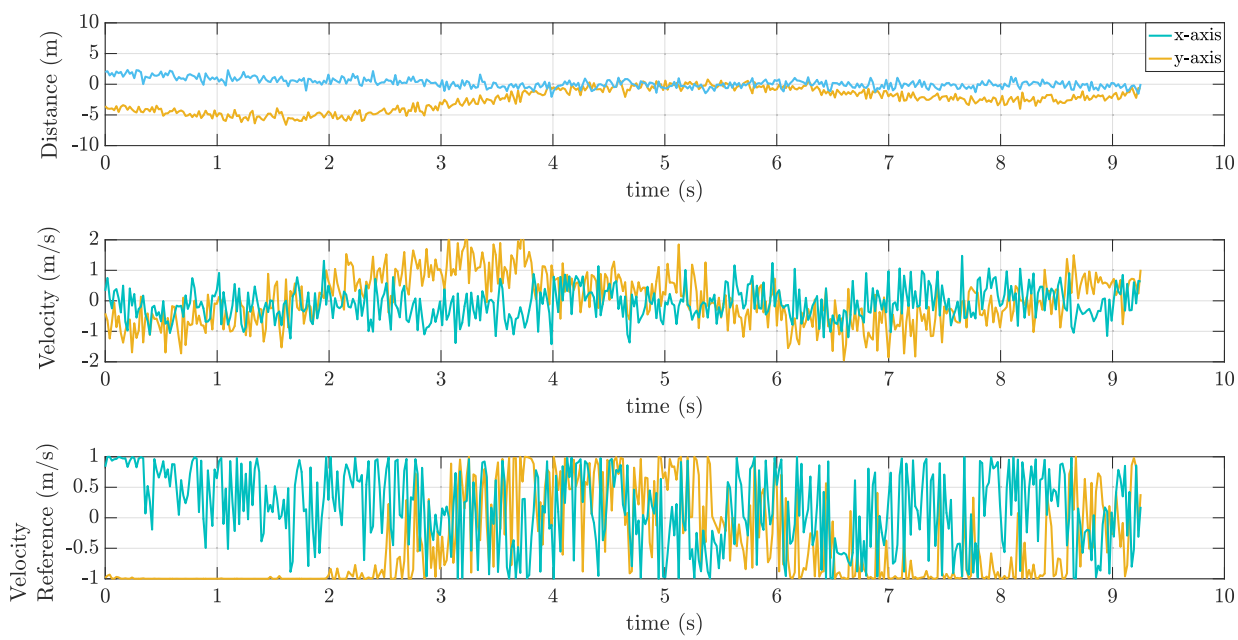

Fig. 6 Partial state and actions signals of episode 4500 (simulated testing phase). (Top) Position of the UAV with respect to the MP with Gaussian noise, in $x$ and $y$ axes. The final position of the UAV with respect to the MP is $0.15 \mathrm{~m}$ and $0.42 \mathrm{~m}$, in $\mathrm{x}$ and $\mathrm{y}$ axes respectively.
(Middle) Velocity of the UAV with respect to the MP with Gaussian noise, in $\mathrm{x}$ and $\mathrm{y}$ axes. (Bottom) Velocity reference commands (actions) generated by the agent, in world coordinates and in $\mathrm{x}$ and $\mathrm{y}$ axes 

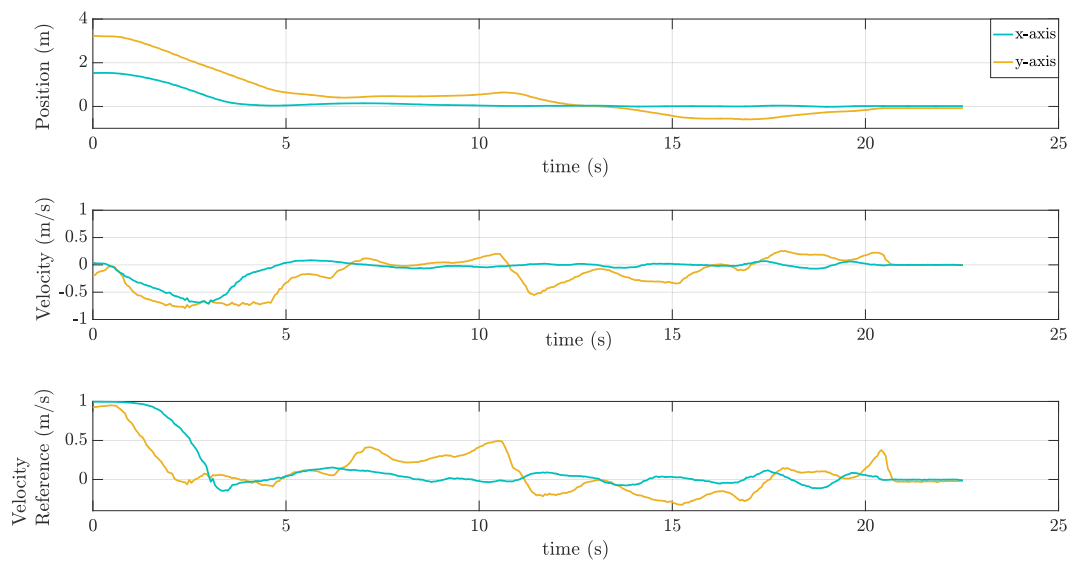

(a) Partial state and actions signals of a real flight (test with a periodic trajectory of the MP). (Top) Position of the UAV with respect to the MP, in $\mathrm{x}$ and $\mathrm{y}$ axes. The final position of the UAV with respect to the MP is $0.01 \mathrm{~m}$ and $-0.07 \mathrm{~m}$, in $\mathrm{x}$ and $\mathrm{y}$ axes respectively. (Middle) Velocity of the UAV with respect to the MP, in $\mathrm{x}$ and $\mathrm{y}$ axes. (Bottom) Velocity reference commands (actions) generated by the agent, in world coordinates and in $\mathrm{x}$ and $\mathrm{y}$ axes.
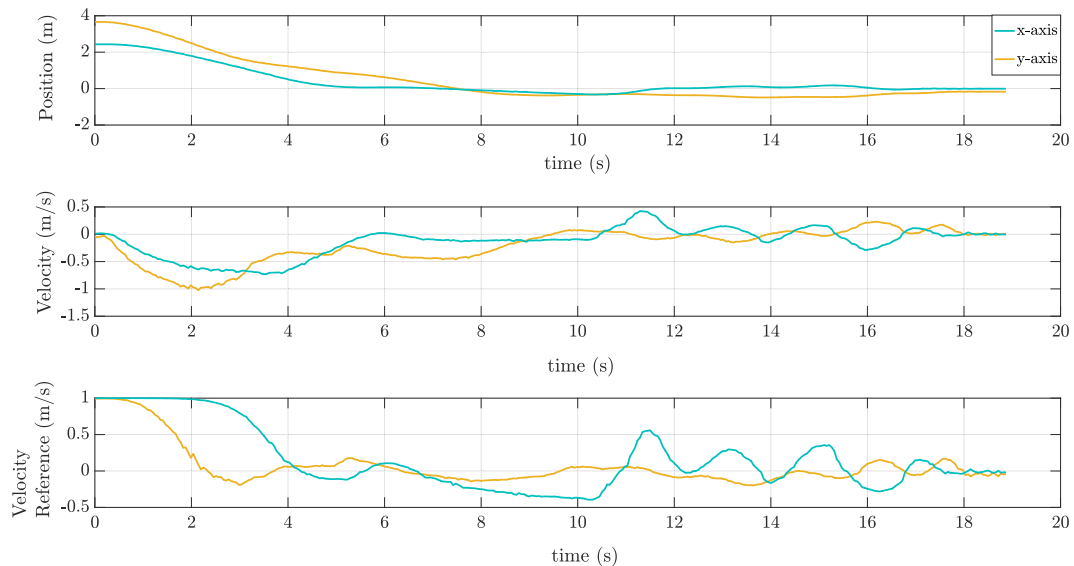

(b) Partial state and actions signals of a real flight (test with a random trajectory of the MP). (Top) Position of the UAV with respect to the MP, in $\mathrm{x}$ and $\mathrm{y}$ axes. The final position of the UAV with respect to the MP is $-0.01 \mathrm{~m}$ and $-0.17 \mathrm{~m}$, in $\mathrm{x}$ and $\mathrm{y}$ axes respectively. (Middle) Velocity of the UAV with respect to the MP, in $\mathrm{x}$ and $\mathrm{y}$ axes.

(Bottom) Velocity reference commands (actions) generated by th agent, in world coordinates and in $\mathrm{x}$ and $\mathrm{y}$ axes.

Fig. 7 Partial state and actions signals of two test episodes (real-flight testing phase) 
Additionally, in order to further validate our selected network from episode 4500, an extensive evaluation in two different scenarios has been performed over 150 test episodes (see Table 1). Both the UAV and the MP start at a random position in each episode (see testing phase area of Section 4.1). Two scenarios have been designed and several metrics have been provided. Slow scenario corresponds to a rectilinear periodic trajectory of the MP with a maximum velocity of $0.4 \mathrm{~m} / \mathrm{s}$. Fast scenario corresponds to a rectilinear periodic trajectory of the MP with a maximum velocity of $1.2 \mathrm{~m} / \mathrm{s}$. tland represents the required time to perform the full landing maneuver (until the UAV touches the MP); $\mathrm{x}$ and $\mathrm{y}$ represent the final position of the UAV with respect to the center of the MP in $\mathrm{x}$ and $\mathrm{y}$ axes, respectively; and the Success Rate (SR) represents the percentage of successful landing trials with respect to the whole set (over 150 episodes). A landing trial is considered successful whether the UAV touches the surface of the moving platform within an area of $1.0 \mathrm{~m} \times 1.0 \mathrm{~m}$ (platform of size $1.2 \mathrm{~m} \times 1.2 \mathrm{~m}$ ). A failure in the landing maneuver is mostly due to the fact that velocity in $\mathrm{z}$ axis is constant and the target MP can become out of range, from where the agent is not able to recover the MP position. Nevertheless, the SR suggests that this approach has succeeded in learning the landing maneuver. Also, both the $t_{\text {land }}$ and the final position of the UAV with respect to the MP shows a proper performance.

The robustness of DDPG algorithm can be further validated in real flights, due to the difference in sensors and dynamics of the UAV used. It has to be noted that no additional tuning of the actions provided by the actor network was required when moving from simulation to real flights. Furthermore, as previously stated, the AscTec Hummingbird included in simulation training and testing phases, and the Parrot Bebop used in real-flight testing have a similar size but the simulated dynamics differ from the real ones. The dynamics of the AscTec Hummingbird were not required to be adjusted in simulation to fit Parrot Bebop dynamics. The explanation of this fact is twofold. First, our approach aims to prove a powerful workflow, where a robot can be trained in simulation and tested in real flights, even when the robot is not being precisely simulated (e.g. different dynamics or autopilot). In this context, the generalization capability of the DDPG algorithm was enough to overcome stated differences. Second, our approach performs high level control (velocity reference control), so that differences in dynamics can be partially absorbed by a proper tuning of the velocity and autopilot controllers.

In Fig. 7, two real-flight plots are shown. In these realflight experiments, the UAV is automatically commanded to land when the altitude with respect to the MP is lower than a certain threshold $t h(t h=35 \mathrm{~cm})$, in order to avoid unsafe maneuvers that do not add value to the final performance.
Also, the UAV is commanded at constant velocity in $\mathrm{z}$ axis. However, due to the Parrot Bebop autopilot design it can sometimes have sudden altitude changes due to missestimation of the UAV altitude in a certain instant (see supplementary video). Figure $7 \mathrm{a}$, shows the performance of the UAV when the MP follows a linear periodic trajectory. This experiment seeks to replicate the scenario which has been used to train the agent in simulation, in order to prove that it is possible to describe a similar behaviour in a real flight. As seen in Fig. 7a, the position of the UAV with respect to the MP converges to the origin and the velocity reference commands generated by the agent are stable with respect to time. Furthermore, in order to test the capability of generalization of the DDPG algorithm, a more complex experiment has been designed. In this new experiment, the MP describes a random trajectory in both $\mathrm{x}$ and $\mathrm{y}$ axes of the horizontal space (ground plane). This scenario has never been experienced by the agent, so that the approach can be proven to be robust and generic enough to overcome the uprising differences, as shown in Fig. 7b. The results shown in Fig. 7b depict a similar convergence, compared to previous results, leading to a proper landing of the UAV on the MP and with a stable generation of actions. The final high level performance of the UAV remains stable, smooth and robust against new experiences. Regarding these results, we can conclude that DDPG algorithm is capable of learning a complex and continuous landing maneuver task. In addition, it is feasible for a UAV to be trained in simulation and tested in the real world without further parameter tuning in a diverse range of conditions.

\section{Conclusions and Future Work}

In this paper, the problem of autonomous landing of a UAV on a moving platform has been solved by means of a deep reinforcement learning algorithm. The state-ofthe-art DDPG algorithm was integrated and adapted into our novel Gazebo-based reinforcement learning simulation framework, enabling the possibility of training complex continuous tasks in a realistic simulation. The UAV landing maneuver task was trained in simulation, and tested in simulation and real flights. This fact has validated a powerful work flow for robotics, where robots can learn in simulation and properly perform in real flights. The experiments have been run in a wide variety of conditions, demonstrating the generalization capability of the approach. To the best of the authors knowledge, this is the first work that addresses the UAV landing maneuver on top of a moving platform by means of a state-of-the-art deep reinforcement learning algorithm, trained in simulation and tested in real flights. Concerning the complexity of the landing problem (and other type of robotic problems), 
other continuous deep reinforcement learning algorithms can be integrated in our reinforcement learning simulation framework, since there is an ongoing innovation in this type of algorithms. As an immediate future work, the altitude ( $z$ axis) can be included in the states and actions spaces and some prediction-based solution can be tested. Furthermore, the input to the algorithm can be changed from a continuous space of variables to raw pixels, in order to test its generalization capability from a higher amount of noisy information.

Acknowledgements This work was supported by the Spanish Ministry of Science (Project DPI2014-60139-R). The LAL UPM and the MONCLOA Campus of International Excellence are also acknowledged for funding the predoctoral contract of one of the authors.

An introductory version of this paper was presented in the 2017 International Conference on Unmanned Aircraft Systems (ICUAS), held in Miami, FL USA, on 13-16 June 2017.

Publisher's Note Springer Nature remains neutral with regard to jurisdictional claims in published maps and institutional affiliations.

\section{References}

1. Rucco, A., Sujit, P.B., Aguiar, A.P., Sousa, J.B., Pereira, F.L.: Optimal rendezvous trajectory for unmanned aerial-ground vehicles. arXiv:1612.06100 (2016)

2. Abadi, M., Agarwal, A., Barham, P., Brevdo, E., Chen, Z., Citro, C., Corrado, G.S., Davis, A., Dean, J., Devin, M., et al.: Tensorflow: Large-scale machine learning on heterogeneous distributed systems. arXiv:1603.04467 (2016)

3. Borowczyk, A., Nguyen, D.-T., Phu-Van Nguyen, A., Nguyen, D.Q., Saussié, D., Ny, J.L.: Autonomous Landing of a multirotor micro air vehicle on a high velocity ground vehicle. In: IFAC World Congress (2017)

4. Ananthakrishnan, U.S., Akshay, N., Manikutty, G., Bhavani, R.R.: Control of quadrotors using neural networks for precise landing maneuvers (2017)

5. Araar, O., Aouf, N., Vitanov, I.: Vision based autonomous landing of multirotor uav on moving platform. J. Intell. Robot. Syst. 85(2), 369-384 (2017)

6. Arora, S., Jain, S., Scherer, S., Nuske, S., Chamberlain, L., Singh, S.: Infrastructure-free shipdeck tracking for autonomous landing. In: 2013 IEEE International Conference on Robotics and Automation (ICRA), pp. 323-330 (2013)

7. Blösch, M., Weiss, S., Scaramuzza, D., Siegwart, R.: Vision based mav navigation in unknown and unstructured environments. In: 2010 IEEE International Conference on Robotics and Automation (ICRA), pp. 21-28. IEEE (2010)

8. Brockman, G., Cheung, V., Pettersson, L., Schneider, J., Schulman, J., Tang, J., Zaremba, W.: Openai gym. arXiv:1606.01540 (2016)

9. Cantelli, L., Mangiameli, M., Melita, C.D., Muscato, G.: Uav/Ugv cooperation for surveying operations in humanitarian demining. In: 2013 IEEE International Symposium on Safety, Security, and Rescue Robotics (SSRR), pp. 1-6. IEEE (2013)
10. Dorigo, M., Colombetti, M.: Robot Shaping: an Experiment in Behavior Engineering. MIT Press, Cambridge (1998)

11. Espié, E., Guionneau, C., Wymann, B., Dimitrakakis, C., Coulom, R., Sumner, A.: Torcs-the open racing car simulator. Available at: http://torcs.sourceforge.net (2005)

12. Falanga, D., Zanchettin, A., Simovic, A., Delmerico, J., Scaramuzza, D.: Vision-based autonomous quadrotor landing on a moving platform

13. Furrer, F., Burri, M., Achtelik, M., Siegwart, R.: Robot operating system (ROS): the complete reference (Volume 1), chap. RotorS-A Modular Gazebo MAV Simulator Framework, pp. 595-625. Springer International Publishing, Cham (2016). https://doi.org/10.1007/978-3-319-26054-9_23

14. Gautam, A., Sujit, P.B., Saripalli, S.: A survey of autonomous landing techniques for uavs. In: 2014 International Conference on Unmanned Aircraft Systems (ICUAS) (2014)

15. Gautam, A., Sujit, P.B., Saripalli, S.: Application of Guidance Laws to Quadrotor Landing. In: 2015 International Conference on Unmanned Aircraft Systems (ICUAS) (2015)

16. Giusti, A., Guzzi, J., Cireşan, D.C., He, F.L., Rodríguez, J.P., Fontana, F., Faessler, M., Forster, C., Schmidhuber, J., Di Caro, G., et al.: A machine learning approach to visual perception of forest trails for mobile robots. IEEE Robotics and Automation Letters 1(2), 661-667 (2016)

17. Gu, S., Lillicrap, T., Sutskever, I., Levine, S.: Continuous deep q-learning with model-based acceleration. In: International Conference on Machine Learning, pp. 2829-2838 (2016)

18. Hu, B., Lu, L., Mishra, S.: Fast, safe and precise landing of a quadrotor on an oscillating platform. In: 2015 American Control Conference (ACC) (2015)

19. Ivakhnenko, A.G.: Polynomial theory of complex systems. IEEE Trans. Syst. Man Cybern. 1(4), 364-378 (1971)

20. Kai, W., Chunzhen, S., Yi, J.: Research on adaptive guidance technology of uav ship landing system based on net recovery. Procedia Engineering 99, 1027-1034 (2015)

21. Kelchtermans, K., Tuytelaars, T.: How hard is it to cross the room?-training (recurrent) neural networks to steer a uav. arXiv: 1702.07600 (2017)

22. Kendoul, F., Ahmed, B.: Bio-inspired taupilot for automated aerial $4 \mathrm{~d}$ docking and landing of unmanned aircraft systems. In: 2012 IEEE/RSJ International Conference on Intelligent Robots and Systems (2012)

23. Kim, D.K., Chen, T.: Deep neural network for real-time autonomous indoor navigation. arXiv: 1511.04668 (2015)

24. Kim, J., Jung, Y., Lee, D., Shim, D.H.: Landing control on a mobile platform for multi-copters using an omnidirectional image sensor. J. Intell. Robot. Syst. 84(1-4), 529-541 (2016)

25. Kober, J., Bagnell, J.A., Peters, J.: Reinforcement learning in robotics: a survey. Int. J. Robot. Res. 32(11), 1238-1274 (2013)

26. Krizhevsky, A., Sutskever, I., Hinton, G.E.: Imagenet classification with deep convolutional neural networks. In: Advances in Neural Information Processing Systems, pp. 1097-1105 (2012)

27. Lee, D., Ryan, T., Kim, H.J.: Autonomous landing of a vtol uav on a moving platform using image-based visual servoing. In: 2012 IEEE International Conference on Robotics and Automation (2012)

28. Lillicrap, T.P., Hunt, J.J., Pritzel, A., Heess, N., Erez, T., Tassa, Y., Silver, D., Wierstra, D.: Continuous control with deep reinforcement learning. arXiv:1509.02971 (2015)

29. Ling, K., Chow, D., Das, A., Waslander, S.L.: Autonomous maritime landings for low-cost vtol aerial vehicles. In: 2014 Canadian Conference on Computer and Robot Vision (2014) 
30. Mnih, V., Badia, A.P., Mirza, M., Graves, A., Lillicrap, T., Harley, T., Silver, D., Kavukcuoglu, K.: Asynchronous methods for deep reinforcement learning. In: International Conference on Machine Learning, pp. 1928-1937 (2016)

31. Mnih, V., Kavukcuoglu, K., Silver, D., Graves, A., Antonoglou, I., Wierstra, D., Riedmiller, M.: Playing atari with deep reinforcement learning. arXiv:1312.5602 (2013)

32. Quigley, M., Conley, K., Gerkey, B., Faust, J., Foote, T., Leibs, J., Wheeler, R., Ng, A.Y.: Ros: an open-source robot operating system. In: ICRA Workshop on Open Source Software, vol. 3, p. 5. Kobe (2009)

33. Rezelj, A.: Autonomous charging of a quadrocopter by landing at a mobile platform (2013)

34. Rodriguez-Ramos, A., Sampedro, C., Bavle, H., Milosevic, Z., Garcia-Vaquero, A., Campoy, P.: Towards fully autonomous landing on moving platforms for rotary unmanned aerial vehicles. In: 2017 International Conference on Unmanned Aircraft Systems (ICUAS), pp. 170-178. IEEE (2017)

35. Sadeghi, F., Levine, S.: rl: real single image flight without a single real image. 12, arXiv:1611.04201 (2016)

36. Sampedro, C., Bavle, H., Rodríguez-Ramos, A., Carrio, A., Fernández, R.A.S., Sanchez-Lopez, J.L., Campoy, P.: A fullyautonomous aerial robotic solution for the 2016 international micro air vehicle competition. In: 2017 International Conference on Unmanned Aircraft Systems (ICUAS), pp. 989-998. IEEE (2017)

37. Sanchez-Lopez, J.L., Fernández, R.A.S., Bavle, H., Sampedro, C., Molina, M., Pestana, J., Campoy, P.: Aerostack: an architecture and open-source software framework for aerial robotics. In: 2016 International Conference on Unmanned Aircraft Systems (ICUAS), pp. 332-341. IEEE (2016)

38. Santana, P., Correia, L., Mendonça, R., Alves, N., Barata, J.: Tracking natural trails with swarm-based visual saliency. J. Field Rob. 30(1), 64-86 (2013)

39. Serra, P., Cunha, R., Hamel, T., Cabecinhas, D., Silvestre, C.: Landing of a quadrotor on a moving target using dynamic imagebased visual servo control. IEEE Trans. Robot. 32(6), 1524-1535 (2016)

40. Shaker, M., Smith, M.N., Yue, S., Duckett, T.: Vision-based landing of a simulated unmanned aerial vehicle with fast reinforcement learning. In: 2010 International Conference on Emerging Security Technologies (EST), pp. 183-188. IEEE (2010)

41. Silver, D., Lever, G., Heess, N., Degris, T., Wierstra, D., Riedmiller, M.: Deterministic policy gradient algorithms. In: Proceedings of the 31st International Conference on Machine Learning (ICML-14), pp. 387-395 (2014)

42. Skoczylas, M.: Vision analysis system for autonomous landing of micro drone. Acta Mechanica et Automatica 8(4), 199-203 (2015)

43. Sutton, R.S., Barto, A.G.: Reinforcement Learning: an Introduction, vol. 1. MIT Press, Cambridge (1998)

44. Todorov, E., Erez, T., Tassa, Y.: Mujoco: a physics engine for model-based control. In: 2012 IEEE/RSJ International Conference on Intelligent Robots and Systems (IROS), pp. 5026-5033. IEEE (2012)

45. Uhlenbeck, G.E., Ornstein, L.S.: On the theory of the brownian motion. Phys. Rev. 36(5), 823 (1930)

46. Vlantis, P., Marantos, P., Bechlioulis, C.P., Kyriakopoulos, K.J.: Quadrotor landing on an inclined platform of a moving ground vehicle. In: 2015 IEEE International Conference on Robotics and Automation (ICRA) (2015)
47. Wenzel, K.E., Masselli, A., Zell, A.: Automatic take off, tracking and landing of a miniature uav on a moving carrier vehicle. J. Intell. Robot. Syst. 61(1-4), 221-238 (2011)

48. Zamora, I., Lopez, N.G., Vilches, V.M., Cordero, A.H.: Extending the openai gym for robotics: a toolkit for reinforcement learning using ros and gazebo. arXiv: 1608.05742 (2016)

49. Zhang, T., Kahn, G., Levine, S., Abbeel, P.: Learning deep control policies for autonomous aerial vehicles with mpc-guided policy search. In: 2016 IEEE International Conference on Robotics and Automation (ICRA), pp. 528-535. IEEE (2016)

Alejandro Rodriguez-Ramos is a Telecommunication Engineer (major in electronic and microelectronic) graduated from Universidad Politécnica de Madrid (UPM, Spain). Currently, he is working as a researcher in the Centre for Automation and Robotics of UPM-CSIC. His $\mathrm{PhD}$ major is Artificial Intelligence (AI) applied to aerial robotics, mainly focused on Deep Learning and Deep Reinforcement Learning techniques. He has participated in international competitions, being awarded with the $2^{\text {nd }}$ prize in the 2017 International Micro Aerial Vehicles competition (IMAV 2017). Previously, he has been working for more than a year in the aerospace sector, contributing to projects of the European Space Agency (ESA). He has broad international experience, since he has worked as a researcher assistant at Aalto University (Espoo, Finland) for a year.

Carlos Sampedro received the Master's degree in Automation and Robotics from the Technical University of Madrid, Madrid, Spain, in July 2014. He is currently working toward the $\mathrm{PhD}$ degree in the Computer Vision and Aerial Robotics Lab belonging to the Centre for Automation and Robotics (UPM-CSIC). His research interests include object detection and recognition using machine learning and deep learning techniques and the development of Deep Reinforcement Learning algorithms applied to aerial robotics. Mr. Sampedro has received a pre-doctoral grant from the Technical University of Madrid in January 2017.

Hriday Bavle is a Ph.D. student at Computer Vision and Aerial Robotics Group (CVAR), Universidad Politecnica de Madrid (UPM), Spain. He received his Bachelors in Aerospace and Masters in Avionics from Amity University, India. Aerial robotics being his core research field, his specialization includes Localization and Mapping techniques applied to UAVs in unknown indoor environments using several computer vision and sensor fusion techniques. He is one of lead developers and testers of the Aerostack software framework developed in the CVAR group. He is also responsible of the entire UAV hardware assembly and maintenance and the lead pilot of the all UAVs within the CVAR group. He has been an active team member in several events of the CVAR group, namely European night of researchers (2015) and the Internationale Micro Aerial Vehicles Competition (IMAV 2016) and team captain at IMAV 2017, where incredible autonomous UAV capabilities using the Aerostack were demonstrated. 
Paloma de la Puente obtained her engineering degree in Automatic Control and Electronics in November 2007 and her Ph.D. in Robotics and Automation in December 2012, both from Universidad Politecnica de Madrid (UPM). She enjoyed a research stay as pre-doctoral visitor at Caltech. After finishing her $\mathrm{PhD}$ she was a post- doctoral researcher at DISAM-UPM and at ACIN Institute of Automation and Control-Vienna University of Technology. She also had professional experience at Ixion Industry and Aerospace and she is currently Assistant Professor at UPM. Her main research interests are related to navigation, mapping, SLAM, spatial cognition, sensor data processing, human-robot interaction for service robotics and systems engineering. She has participated in several national and European projects and also in international robotics competitions.
Pascual Campoy is Full Professor on Automatics at the Universidad Politécnica Madrid UPM (Spain) and visiting professor in TUDelft (The Netherlands), he has also been visiting professor at Tong Ji University (Shanghai-China) and Q.U.T. (Australia). He currently lectures on Control, Machine Learning and Computer Vision.

$\mathrm{He}$ is leading the Research Group on "Computer Vision and Aerial Robotics" at U.P.M. within the Centre of Automatics and Robotics (C.A.R.), whose activities are aimed at increasing the autonomy of the Unmanned Aerial Vehicles (UAV) by exploiting the powerful sensor of Vision, using cutting-edge technologies in Image Processing, Control and Artificial Intelligence.

He has been head director of over $40 \mathrm{R} \& \mathrm{D}$ projects, including R\&D European projects, national R\&D projects and over 25 technological transfer projects directly contracted with the industry. He is author of over 200 international scientific publications and nine patents, three of them registered internationally. He is awarded several international prices in UAV competitions: IMAV12, IMAV13, IARC14, IMAV16 and IMAV17. 


\section{Publication II}

(C) 2019 by the authors. Licensee MDPI, Basel, Switzerland. This article is an open access article distributed under the terms and conditions of the Creative Commons

Attribution (CC BY) license (http://creativecommons.org/licenses/by/4.0/).

Rodriguez-Ramos, A., Alvarez-Fernandez, A., Bavle, H., Campoy, P., and How, J. P. (2019). Vision-based multirotor following using synthetic learning techniques. Sensors, 19(21), 4794. doi:10.3390/s19214794. JCR (2019): 3.275, Instruments and Instrumentation (Q1), Engineering, Electrical \& Electronic (Q2). 



\title{
Vision-Based Multirotor Following Using Synthetic Learning Techniques
}

\author{
Alejandro Rodriguez-Ramos ${ }^{1, *(\mathbb{D}}$, Adrian Alvarez-Fernandez ${ }^{2}$, Hriday Bavle ${ }^{1}$, \\ Pascual Campoy ${ }^{1}$ (D) and Jonathan P. How ${ }^{3}$ (D) \\ 1 Computer Vision and Aerial Robotics group, Centre for Automation and Robotics, Universidad Politécnica \\ de Madrid (UPM-CSIC), Calle Jose Gutierrez Abascal 2, 28006 Madrid, Spain; hriday.bavle@upm.es (H.B.); \\ pascual.campoy@upm.es (P.C.) \\ 2 Artificial Intelligence group, University of Groningen, 9712 Groningen, The Netherlands; \\ a.alvarez.fernandez@student.rug.nl \\ 3 Aerospace Controls Laboratory, Massachusetts Institute of Technology (MIT), 77Mass. Ave., \\ Cambridge, MA 02139, USA; jhow@mit.edu \\ * Correspondence: alejandro.rramos@upm.es
}

Received: 9 August 2019; Accepted: 30 October 2019; Published: 4 November 2019

\begin{abstract}
Deep- and reinforcement-learning techniques have increasingly required large sets of real data to achieve stable convergence and generalization, in the context of image-recognition, object-detection or motion-control strategies. On this subject, the research community lacks robust approaches to overcome unavailable real-world extensive data by means of realistic synthetic-information and domain-adaptation techniques. In this work, synthetic-learning strategies have been used for the vision-based autonomous following of a noncooperative multirotor. The complete maneuver was learned with synthetic images and high-dimensional low-level continuous robot states, with deep- and reinforcement-learning techniques for object detection and motion control, respectively. A novel motion-control strategy for object following is introduced where the camera gimbal movement is coupled with the multirotor motion during the multirotor following. Results confirm that our present framework can be used to deploy a vision-based task in real flight using synthetic data. It was extensively validated in both simulated and real-flight scenarios, providing proper results (following a multirotor up to $1.3 \mathrm{~m} / \mathrm{s}$ in simulation and $0.3 \mathrm{~m} / \mathrm{s}$ in real flights).
\end{abstract}

Keywords: multirotor; UAV; following; synthetic learning; reinforcement learning; deep learning

\section{Introduction}

Currently, learning techniques aided by synthetic information are increasingly popular in the robotics field, mostly due to the lack of available real-domain data information for application-specific tasks, for example, low-level motion control for complex robot dynamics [1-3] or vision-based strategies for unusual scenarios [4-6]. In addition, with the rise of deep learning [7] and multiple-layer models, the amount of data required for the stability of learning and model generalization has grown several orders of magnitude, which makes dataset generation harder. State-of-the-art reinforcement-learning techniques are also still notably sample-inefficient [8], which leads to needing a high number of agent-environment interactions, which is normally not possible in the real world due to system stability, sample randomness or power availability. In this context, in order to achieve scalability for both deep- and reinforcement-learning techniques, the exploration of synthetic-learning strategies in robotics is a matter of importance that is not yet resolved. This paradigm is potentially valuable when annotated datasets are not available and/or there is highly nonlinear mapping between several 
heterogeneous information sources, for example, navigation-throughout-cluttered indoor and outdoor environments using simulated image cues [9].

In the context of synthetic-data generation by robotic simulators, there is an explicit differentiation between robot-dynamics simulation and photorealistic-image generation and they are normally treated separately. Realistic simulation of robot dynamics is important in the field of imitationor reinforcement-learning algorithms, that is, more realistic models and/or physics can lead to more effective performance in the real world. In this regard, novel system dynamics can be realistically simulated to be controlled with learning-based (or classical) approaches and without the necessity of building the actual robot. Although there are inconsistencies between virtual and real models, differences between simulated and real systems can be tackled with several methods (e.g., References [1-3]). Within the scope of the generation of photorealistic images, there is still a clear ceiling in the achieved realism for current simulators. Light, polygons and material simulations have intrinsic limitations that can be very evident for a human observer. Nevertheless, they can be extremely useful in the field of vision-based strategies, such as object detectors, along with other transfer-learning or domain-adaptation techniques (e.g., References [10-12]).

In this scenario, a multirotor can be a suitable robotic test bench to validate this type of synthetic technique for robotic applications. Their inherent open-loop system instability, which can create a stressful scenario for the technique under research and their low-cost RGB camera, which can lead to a wide variety of applications, make this platform a suitable target for novel motion-control research, as well as for vision-based strategies. In this respect, the versatility of this platform and the lack of complete learning-based techniques aided by synthetic data compose the base motivation for the studied application case in this work. Indeed, the multirotor following task introduced in this study was proposed as a baseline task due to its challenging nature and increasing interest of the research community (e.g., International robotics competition: https://www.mbzirc.com/). At the same time, since multirotors have become the center of attention of many industrial automation processes, the capacity of multirotor detection with a low-cost sensor and the ability to follow a potential noncooperative multirotor is an interesting topic of research.

In particular, this paper explores an scenario where a noncooperative target multirotor has the ability to move in the 3-Degrees of Freedom (DOF) of space and the follower multirotor, which implemented our approach, specifically relies on its low-level attitude Flight Controller (FC) and its monocular RGB camera to accomplish the complete following maneuver. We focused on a novel object-following case where the follower RGB camera was also able to move in two directions of space (camera gimbal pan and tilt), providing higher dimensionality and versatility to the approach. The entire behavior was learned with synthetic data by means of two strategies based on deep and reinforcement learning for multirotor detection and motion control, respectively.

\section{Contribution}

In this study, monocular vision-based following of a noncooperative multirotor using synthetic-learning techniques is presented. The aim was to establish a complex real-world multirotor application based on synthetic data, by means of deep- and reinforcement-learning methods. The main contributions of this work are listed below:

- A robust detection technique was trained with a novel approach based on synthetic photorealistic images generated by a commercial game engine. The synthetic multirotor image dataset, utilized in this work for detector training, was also released as an open-source dataset.

- Problem formulation under the reinforcement-learning framework was designed in order to achieve learning convergence for a motion-control agent with a state-of-the-art deep reinforcement-learning algorithm and within the context of high-dimensional continuous state and action spaces. The agent was trained in a simulated environment and tested in real-flight experiments (refer to Figure 1).

- A novel motion-control strategy for object following is introduced where camera gimbal movement is coupled with multirotor motion during multirotor following. 


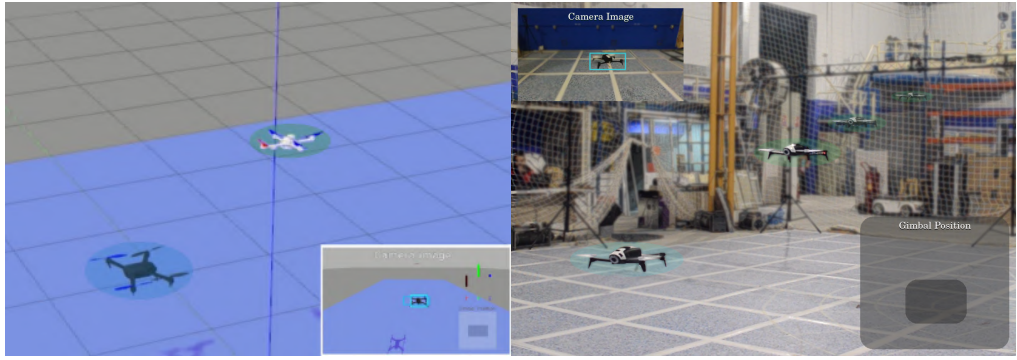

(a)

(b)

Figure 1. Image composition of a noncooperative multirotor (light-blue) followed by another multirotor that implemented our synthetic-learning-based approach (light-green) in the (a) simulated and (b) real experimentation environment.

Additionally, real-flight tests were performed in a broad variety of conditions in order to validate the virtual-to-real approach and with low-cost on-board sensors as single raw data inputs. Results prove that our present framework can be used for vision-based tasks in a real flight using synthetic data. The remainder of the paper is organized as follows: Section 4 describes our approach and provides a detailed explanation about the multirotor detector and the motion-control policy, explaining the problem formulation and the system architecture. Section 5 outlines the carried-out experiments and their corresponding results and Section 6 remarks on and discusses the most relevant experimentation outcomes. Finally, Section 7 concludes the paper and indicates future lines of research.

\section{Related Work}

In this paper, the task and techniques under study were approached with strategies of diverse nature and, due to this, there are several related works that are adjacent to the case under study. In the following section, the key aspects of the most relevant related works are described.

\subsection{Autonomous Aerial Pursuit}

Autonomous aerial pursuit, that is, Unmanned Aerial Vehicle (UAV)-to-UAV pursuit, has been an underlying field of research during the last decade. This line of research has not been the focus of UAV research but has provided theoretical contributions mostly validated on numerical simulations. Hence, most innovations assumed ideal data information sources and were tested only by means of numerical simulations [13-17].

In References [14-16], the target UAV state was assumed to be known and considered cooperative under the case of study. In Reference [17], a sliding-mode approach with proper Lyapunov function was explored and validated on numerical simulations. In Reference [16], both pursuit algorithms and vision-based theoretical strategies were explored under the assumption of ideal sensors and validated on numerical simulations. A cooperation strategy for multiple UAVs to pursue a target moving in an adversarial environment where threat exposure should be minimized was studied in Reference [13]. A probabilistic model of the environment was provided and the information exchange between the cooperative UAVs is assumed to be available in a numerical simulation.

\subsection{Monocular Vision-Based Object Following}

Several works researched the topic of vision-based autonomous object following with UAVs. Most of them explored classical control schemes (e.g., Proportional Integral Derivative (PID) control [18]) with classical computer-vision techniques (e.g., color-based detectors [19]). 
In Reference [20], a nonlinear adaptive observer was used for target-object estimation and a guidance law for target-object tracking was developed. In this case, the vision sensor was modeled and the complete approach was validated under numerical simulations.

Some approaches rely on an online user-defined Region-of-Interest (RoI) within the image plane during the execution of a real flight in order for the UAV to be able to follow a tracked object $[18,21,22]$. In Reference [18], a PID control scheme was used to control a multirotor aerial robot based on the OpenTLD [23] tracker in the image plane. In Reference [21], the authors developed a novel frequencyand image-plane-domain tracker with latter pose estimation of the object and standard PID control. In Reference [22], a state-of-the-art tracker [24] was used and a standard PID control scheme within the image plane was established. The stated work also performed real-flight validation tests and developed a novel camera handover strategy in order to enable long-term operation through several UAVs. As other close related works, some strategies use learning techniques for orientation tracking with respect to image patches based on state-of-the-art detectors and trackers $[25,26]$.

Furthermore, some techniques carried out specific-object detection in conjunction with continuous-tracking strategies, such as Kalman or particle filters [19,27,28]. In References [27,28], a strategy for human following was designed and tested in real flights. In Reference [27], a miniature robotic blimp was used as the robotic platform, with human-pose estimation and standard PID control. In Reference [28], human tracking was carried out over a long period of time and long distances by means of active infrared markers and the estimation of the pose of both human and UAVs without calibrated or stationary cameras. In Reference [19], a color-based detector fed a particle filter that aided a pose-based PID control scheme.

Finally, some other strategies aimed at separately controlling a camera gimbal for object tracking, in which the detected object is kept centered in the image plane over time [29,30]. In Reference [29], a ground-based camera gimbal is able to track a remote object. In Reference [30], a camera gimbal was mounted as payload on a fixed-wing UAV for target tracking during the execution of the test flights.

\subsection{Reinforcement Learning for Real-World Robotics}

Several state-of-the-art techniques aim at training reinforcement-learning agents with synthetic data but by targeting their deployment in the real world. Some techniques focus on learning high-dimensional system dynamics for motion control [1-3]. The authors of Reference [1] utilized precise simulations and randomized system dynamics for quadruped locomotion learning with continuous states and actions. In Reference [2], high-level velocity control was learned with ground-truth simulations in order to develop a UAV task-specific landing maneuver on top of a moving platform. Validation tests were carried out through a motion-capture system in the real world. In Reference [3], the dynamics of a multirotor UAV were precisely simulated in order to train a reinforcement-learning agent for low-level attitude control. The learned policies were directly validated in the real world with a motion-capture system.

On the other hand, some strategies intend to overcome the virtual-to-reality gap for vision-based applications [9,31-35]. In Reference [9], synthetic-data adaptation was performed using an application-dependent Cycada pipeline for ground-robot navigation. In Reference [31], domain-randomization techniques were used to train a deep reinforcement-learning algorithm for dexterous in-hand manipulation. The authors of Reference [32] randomized simulated images to generate a canonical environment for hand manipulation.

Moreover, multirotor-related vision-based applications have also been studied [33-36]. In Reference [33], a color-based detector and an image-based deep reinforcement-learning policy controller were utilized for object following with an off-the-shelf multirotor. In Reference [36], human commands were mixed with reinforcement-learning exploration-exploitation actions in order to improve safety for multirotor navigation. The authors of Reference [35] used the Monte Carlo policy-evaluation method for vision-based navigation learning with synthetic images. 
In Reference [34], small-size multirotor navigation was achieved through a combination of real-world data to learn about robot dynamics and synthetic data for vision-based collision avoidance.

\subsection{Multirotor In-Flight Detection and Tracking}

Multirotor in-flight detection and tracking is increasingly becoming a topic of interest for the research community since the successful application of multirotor UAVs in several automation processes in the industry. Most strategies rely on motion detection during flight for a relatively reduced subset of UAV types [10-12,37,38]. In Reference [10], Convolutional Neural Networks (CNNs) were used for camera-motion estimation and temporal information was exploited to detect UAVs and aircrafts. In Reference [11], pixels corresponding to the background in the image were estimated and a Kalman filter was utilized to track motion of a UAV. In Reference [12], UAV detection and tracking required the exchange of information between both UAVs. The authors of Reference [37] explored Haar-like features, Histogram of Gradients (HOG) and Local Binary Patterns (LBP) using cascades of boosted classifiers for outdoor UAV detection and tracking. In Reference [38], extensive flight experimentation was carried out with real-time performance for general object tracking.

\subsection{Object Detection with Synthetic Images}

Deep learning is de facto the most used technique for object detection. Nevertheless, it usually requires a large amount of domain-specific data to achieve generalization. In this context, the research community is exploring the incorporation of synthetic images into the training process due to their availability and capabilities [4-6,39-42]. In Reference [4], synthetic images were optimized to match object-detector-extracted features. These images were used for real-image dataset augmentation [4]. The authors of Reference [5] randomized simulated images for 3D object localization; objects were composed of low-dimensional feature textures and shapes.

In Reference [40], synthetic images were generated by adding background and textures to simple 3D Computer-Aided Design (CAD) models. Features were extracted with a CNN trained on ImageNet and a classifier was trained for the final stage. In Reference [6], adversarial training was used to train Faster R-CNN [43] for cross-domain adaptation. The domains were adapted at the image level (e.g., image style and illumination) and instance-level representation (e.g., object appearance and size shift). In Reference [39], simple synthetic objects were detected through backbone-network (feature extractor) weight freezing and fine-tuning the last layers. Validation tests were performed with Faster R-CNN, R-FCN [44] and Mask R-CNN [45]. In Reference [41], adversarial training was used for simulated-to-real domain transferring. The dataset was generated through synthetic-image refinement and automated labeling. Finally, novel approaches showed outstanding performance when trained with small real-world datasets, outperforming previous pretrained architectures [46], such as SSD or Faster R-CNN. These strategies are potentially usable in the field of synthetic-dataset integration .

\section{Vision-Based Multirotor Following Approach}

In this paper, the vision-based autonomous following of a Noncooperative Multirotor (NC-M) is presented. Our approach is mainly composed of a multirotor detector, an image-based tracker and motion-control policy.

\subsection{Multirotor Detector}

A deep-learning-based detector was trained by means of synthetic multirotor images with a simple background (see Section 5.2.1) and with the aim of extracting an accurate RoI of the NC-M. The detector is able to generalize enough from synthetic multirotor images and provide state-of-the-art accuracy and inference time. The detector used in this study was a custom implementation of the one-stage RetinaNet detector [47], which is a single unified network composed of a backbone network and two task-specific subnetworks (refer to Figure 2). The backbone is responsible for computing a convolutional and pyramidal feature map over an entire input and is an off-the-shelf convolutional network. In this work, 
ResNet50 was selected as the full network backbone in order to find a balanced trade-off between accuracy and image throughput [48].

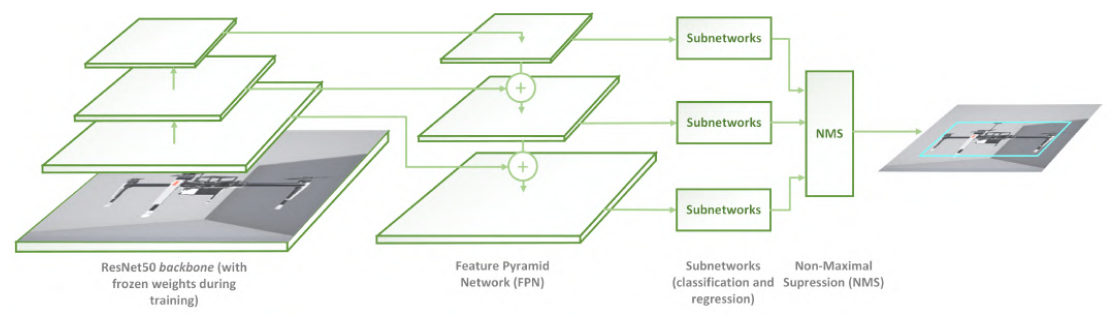

Figure 2. Architecture of proposed multirotor detector, based on RetinaNet architecture, with ResNet50 backbone and five levels for Feature Pyramid Network (FPN; fewer levels represented in figure for the sake of simplicity). Backbone weights were frozen during training. Final subnetworks were fine-tuned with pure synthetic multirotor images.

The key aspects for RetinaNet to be suitable for learning new pure synthetic classes are its rich feature maps, generated by its Feature Pyramid Network (FPN) architecture and its Focal Loss (FL) design, which is a specific case of a weighted cross-entropy loss function. RetinaNet was also proven to be the best architecture in terms of accuracy and speed, compared with Faster R-CNN, R-FCN and other state-of-the-art architectures, in the wide variety of conditions presented in Reference [47]. Our approach incorporated five-layer levels from the backbone for FPN feature-map generation. FL minimizes the effect of large class imbalance (diminishing the influence of already well-learned classes) and encourages learning those samples that have not yet been properly learned (i.e., particular losses of already learned samples can overwhelm whole loss computation), as shown in Equations (1) and (2).

$$
\begin{gathered}
F L\left(p_{t}\right)=-\alpha_{t}\left(1-p_{t}\right)^{\eta} \log \left(p_{t}\right) \\
p_{t}=\left\{\begin{array}{ll}
p & \text { if } y=1 \\
1-p & \text { otherwise }
\end{array},\right.
\end{gathered}
$$

where $\eta$ is the focusing parameter and $\alpha_{t}$ is the classical $\alpha$-balanced loss parameter [47]. This model architecture enables the detection (RoI) of a real NC-M within the image plane, which can be used by the next stages of the approach.

\subsection{Image-Based Tracker}

The stated multirotor detector can infer the RoI of the detected NC-M. Nevertheless, due to the multirotor perspective, illumination changes or glitches in the video feed, the multirotor detector could fail to regress a RoI, even when the NC-M is present in the image and the detector is able to run in real time. In this scenario, in order to provide a continuous RoI of the NC-M and to improve overall performance, our approach incorporated an adapted implementation of the state-of-the-art ECO tracker [49] that can track the RoI of the NC-M in every camera frame during the following maneuver.

The key aspects of the ECO tracker that make it suitable for these dynamic-feature frames are its robust hand-crafted features for tracking and its factorized convolution design. The hand-crafted features are computationally efficient and robust. The factorized convolution operator dramatically reduces the number of parameters of the Discriminative Correlation Filter (DCF), allowing for real-time tracking $(>20 \mathrm{~Hz})$ on an average CPU. In this context, the tracker is able to provide a RoI at every time step. However, when the detector provides a new RoI (with a predefined threshold of uncertainty that assures robustness), the current tracker RoI is updated for further tracking. 


\subsection{Motion-Control Policy}

Deep reinforcement-learning techniques were applied for learning the multirotor motion-control policy for object following. The complete learned behavior is challenging due to the coupled nature of actuation-response of the system, that is, the multirotor system was coupled with the camera gimbal actuation since different trajectories of actuation (in both the multirotor and the camera gimbal) could lead to the same result in the image plane. This characteristic of the system is further explored in Section 5 .

Considering the reinforcement-learning framework, an agent and an environment compose the two main elements that are defined to interact with each other. The stated interaction is carried out through agent actions that occur in an instantaneous effect in the environment, (returning the effect consequence back to the agent, that is, agent state) and through environment rewards that guide the evolution of the agent behavior. Thus, in the reinforcement-learning paradigm, an agent is determined to generate a policy $\pi\left(s_{t}\right)$ in order to maximize its expected accumulated reward $R_{t}$ through the execution of an action $a_{t} \in \mathbb{A}$ for each state $s_{t} \in \mathbb{S}$ at any step in time $t$. The expected return, given a state and an action in time $t$, is

$$
Q^{\pi}\left(s_{t}, a_{t}\right)=\mathbb{E}_{\pi}\left[R_{t} \mid s_{t}, a_{t}\right]
$$

where $Q^{\pi}\left(s_{t}, a_{t}\right)$ represents the action-value function. A broadly used reinforcement-learning approach is learning the stated action-value function by means of an optimization process that has as its main objective to estimate the optimal Q-function (which, in the context of discrete action spaces, leads to an optimal policy of the agent). Other algorithms aim at directly estimating the agent optimal policy $\pi^{\star}\left(s_{t}\right)$ by means of the parallel estimation of the Q-function (or, equivalently, the V-function [50]) and the computation of the policy gradient [51]. These methods, that follow the actor-critic paradigm and are compatible with continuous state and action spaces are known as policy-gradient methods, such as Deep Deterministic Policy Gradients (DDPGs) [51] or Trust-Region Policy Optimization (TRPO) [52]. Other recent algorithms, which compose a family of natural policy-gradient algorithms named Proximal Policy Optimization (PPO) [53], include a surrogate objective function for optimization. This family of algorithms lead to a reduction of complexity and an increase of both sample efficiency and performance. Stated increase in performance was taken into consideration for the application under study due to its high-dimensional nature of state and action spaces (refer to Section 4.3.1). Since PPO aims at directly optimizing an objective function for the policy, an instant update of the policy is controlled in order to avoid divergence and to assure optimal convergence. The strategies for constraining the policy update are diverse and a matter of research. Nevertheless, the most common strategies are based on Kullback-Leibler divergence (KL-divergence) penalty and on direct-clipping penalty (see Equation (4)).

$$
\mathcal{L}_{\theta_{k}}^{C L I P}=\mathbb{E}_{\tau \sim \pi_{k}}\left[\sum_{t=0}^{T}\left[\min \left(r_{t}(\theta) \hat{A}_{t}^{\pi_{k}}, \operatorname{clip}\left(r_{t}(\theta), 1-\epsilon, 1+\epsilon\right) \hat{A}_{t}^{\pi_{k}}\right)\right]\right]
$$

where $\mathcal{L}_{\theta_{k}}^{C L I P}$ represents the objective function for policy-weight update, $r_{t}(\theta)$ is the new-old policy ratio, $\hat{A}_{t}^{\pi_{k}}$ is the advantage function for policy update at time $t$ and $\epsilon$ is the clipping constraint. In this work, due to computation simplicity and performance increase, PPO with a clipping penalty was selected to train the agent to accomplish the image-based multirotor following maneuver with continuous state and action spaces.

\subsubsection{Problem Formulation}

In our problem formulation, the reinforcement-learning agent perceives a continuous state $s_{t} \in \mathbb{S}$ at time $t(5)$.

$$
\mathbb{S}=\left\{e_{c x}, e_{c y}, e_{\text {area }}, \dot{e}_{c x}, \dot{e}_{c y}, \dot{e}_{\text {area }}, \Theta_{x}, \Theta_{y}\right\},
$$


where $e_{c x}, e_{c y}$ and $e_{\text {area }}$ represent the normalized error of the current RoI with respect to the target RoI center position (in $\mathrm{x}$ and $\mathrm{y}$ axes) and area; $\dot{e}_{c x}, \dot{e}_{c y}$ and $\dot{e}_{a r e a}$ represent the normalized difference of errors $\left(e_{c x}, e_{c y}\right.$ and $\left.e_{a r e a}\right)$ with respect to the previous time step and $\Theta_{x}$ and $\Theta_{y}$ are the normalized angular states of the camera gimbal in the current time step. The state is represented in the Camera (C) frame of reference (see Figure 3 ) and $\mathbb{S} \in[-1,1]$. A virtual camera gimbal was added to the multirotor simulation in order to meet real platform specifications.

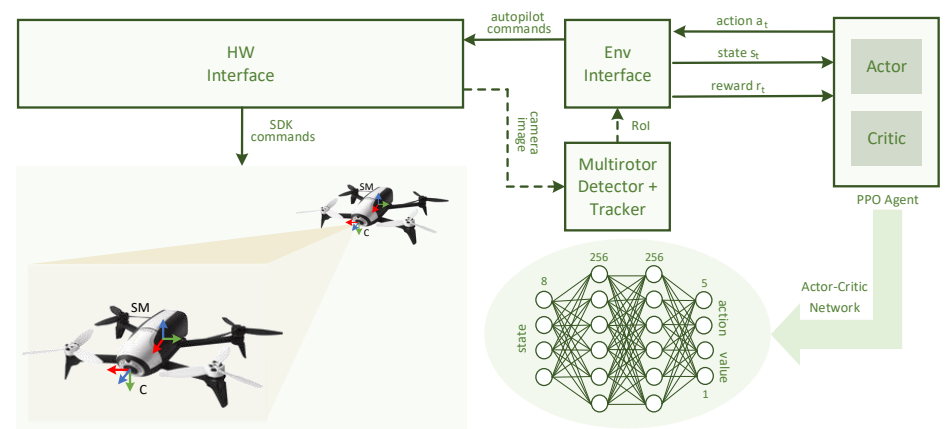

Figure 3. Architecture of proposed deep- and reinforcement-learning-based multirotor following system. Every involved frame of reference and architecture component is depicted. Actor-critic network, which is in charge of providing the final behavior, is structurally represented. Stabilized Multirotor (SM) frame of reference is stabilized in pitch and roll and attached to the multirotor body structure. Multirotor detector and tracker were only included in real-flight testing (dashed lines). In simulated experiments, a ground-truth Region of Interest (RoI) was generated based on projection of NC-M 3D points.

The reinforcement-learning agent has the ability to perform a continuous action $a_{t} \in \mathbb{A}$ at time $t$, as represented in Equation (6).

$$
\mathbb{A}=\left\{\theta, \phi, \dot{z}, \dot{\Theta}_{x}, \dot{\Theta}_{y}\right\},
$$

where $\theta$ and $\phi$ represent the multirotor pitch and roll absolute angles, respectively; $\dot{z}$ represents multirotor altitude velocity; and $\dot{\Theta}_{x}$ and $\dot{\Theta}_{y}$ represent the camera gimbal angular difference of angles in the $x$ and $y$ axes, respectively. The action space is represented in the Stabilized Multirotor (SM) frame of reference for $\theta, \phi$ and $\dot{z}$ and in the $C$ frame of reference for $\dot{\Theta}_{x}$ and $\dot{\Theta}_{y}(\mathbb{A} \in[-1,1])$. The actions are directly forwarded to the multirotor FC as an input through SDK commands, avoiding postprocessing or filtering.

An important component in reinforcement-learning formulation is the reward function $r$, due to the high sensitivity of the current techniques to the reward-function design. Although there are some techniques that are able to deal with low-frequency and sparse-reward functions [54], most of the techniques require a more behavior-guided design. In this scenario, a well-suited reward function can decrease training times but, conversely, a weak design can introduce human bias in the final policy or even completely prevent the agent from learning a stable policy. In our presented formulation, the reward function was designed in a scheduled trend, by rewarding the main application goal higher, which keeps the target multirotor in the image plane but at the same time, by encouraging safe and smooth movements. Resulting reward function $r$ is 


$$
\begin{aligned}
& r= \begin{cases}r_{1}=-100 & \text { if } c x \text { or } c y \\
& \text { out of image } \\
r_{2}=-100 & \text { if area }<350 \mathrm{px}^{2} \\
r_{3} & \text { otherwise }\end{cases} \\
& r_{3}=\operatorname{shaping}[t]-\operatorname{shaping}[t-1] \\
& \text { shaping }[t]=\mathrm{p}_{\text {center }}[t]+\mathrm{p}_{\text {gimbal }}[t] \\
& +\mathrm{p}_{\text {area }}[t]+\mathrm{p}_{\text {velocity }}[t] \\
& \mathrm{p}_{c e n t e r}[t]=-g_{1} \sqrt{e_{c x^{2}}+e_{c y}{ }^{2}} \\
& \mathrm{p}_{\text {gimbal }}[t]=-g_{2} \sqrt{\Theta_{x}^{2}+\Theta_{y}^{2}} \\
& \mathrm{p}_{\text {area }}[t]=-g_{3}\left|e_{\text {area }}\right| \\
& \mathrm{p}_{\text {velocity }}[t]=-g_{4} \sqrt{\dot{e}_{c x}^{2}+\dot{e}_{c y}^{2}}
\end{aligned}
$$

where $c x, c y$ and area represent the center (in $\mathrm{x}$ and $\mathrm{y}$ axes) and the area of the current RoI, respectively; $g_{1}, g_{2}, g_{3}$ and $g_{4}$ are experimentally defined constants (100,65, 50,30, respectively); $r_{1}$ and $r_{2}$ prevent the agent from exploring states out of a certain volume with respect to the target NC-M, based on image coordinates; and $r_{3}$ informs the agent about its instantaneous progress and helps speed up learning [55].

Furthermore, in the shaping component an explicit distinction was included in the importance of minimizing the relative image position between current and target RoI centers, the absolute position of the camera gimbal, the current and target RoI area ratio and the error velocities (each variable was weighted by a different coefficient $g_{1}, g_{2}, g_{3}$ and $g_{4}$ ). In this trend, the agent is encouraged to coarsely learn to minimize the position of the current RoI with respect to the target RoI, which retains the target NC-M within the camera Field of View (FOV). Subsequently, the agent is encouraged to optimize its behavior in order to keep the camera gimbal in a centered position (angles close to zero) and to finally keep a certain distance with respect to the target multirotor (RoI area directly related to the distance) as well as to decrease image velocities. Particularly, we found out that the incorporation of $\Theta_{x}$ and $\Theta_{y}$ components, weighted by $g_{2}$ coefficient, is a determinant for learning convergence. As stated, this component of the reward function encourages the agent to keep the camera gimbal in a centered position during the execution of the maneuver, diminishing the uncertainty of the solution space and making the desired behavior more explicit, that is, centering the camera gimbal provides the RL-M with more reaction time in case of sudden movements by the NC-M. Finally, the final reward was not normalized. Although $\mathrm{p}_{\text {center }}[t], \mathrm{p}_{\text {gimbal }}[t], \mathrm{p}_{\text {area }}[t]$ and $\mathrm{p}_{\text {velocity }}[t]$ by far exceeded the unitary value during training, the final computation of reward $r_{3}$, which informs the agent about its instantaneous progress, did not. In the context of this application and reward-function design, final reward $r_{3}$ is in the decimal order of magnitude, with values near the unit. Thus, taking this into consideration and the algorithm involved, reward normalization was not required.

\subsubsection{System and Network Architecture}

A versatile system architecture was designed and implemented, taking the standardization of the virtual and real contexts as the main consideration. In this scenario, most of the component interfaces were shared for both simulated and real-flight experiments. A global overview of the system architecture is depicted in Figure 3. From the side of the agent, the PPO component, which had the actor-critic network as its motion-policy representation model, was wrapped with an ROS [56] interface. This design not only increased the similarity between the virtual and real contexts but also reduced the friction of interaction with robotic components, since ROS is the most common 
middleware in the robotic ecosystem. Conversely, the implemented environment interface is in charge of parsing the raw information (target NC-M RoIs and angular states of the camera gimbal) in order to properly adapt it to the reinforcement-learning formulation (refer to Section 4.3.1). It is also in charge of interacting through SDK commands with the hardware interface, which can be real or simulated due to standard implementation. The RoI of the target NC-M was generated based on the projection of the multirotor ground-truth $3 \mathrm{D}$ points and the intrinsic camera parameters for the simulated environments and based on the multirotor detector, described in Section 4.1, for real-flight experiments. Hence, in order to send state $s_{t}$ to the PPO agent, the environment interface performs all the required processing by relying on current and target RoIs.

The actor-critic neural network is a feed-forward neural network with two hidden layers of 256 units each. The activation function of each unit of a hidden and output layers is a hyperbolic tangent $(\tanh )$. The input- and output-layer dimensions of the actor-critic network are based on the state, action and value dimensions ( 8 and $5+1$ units), respectively (see Figure 3 ). The activation function of the output layer is bounded to the range of $[-1,1]$ and is provided by a linear unit for the value, to output an estimation of the V-function, used to compute an advantage function [53]. It has to be remarked that other values of the number of hidden layers and/or hidden units have been tested. Nevertheless, the stated neural-network structure composes the minimum size in terms of hidden layers and units per layer, which allows for learning stability in the conditions described in this work. For instance, network models with 128 units and two hidden layers did not provide proper results.

\section{Experiments}

As supplementary material, the video of the experiments can be found in https:/ vimeo.com/ 352940150, and the stated open-source dataset in https://bitbucket.org/alejodosr/uav-dataset.

\subsection{Experiment Setup}

The environment and hardware interfaces were been implemented in c++11 and the neural networks were trained with the Tensorflow (https: / / www.tensorflow.org/) library. The multirotor detector was migrated to $\mathrm{c}++11$ for fast-inference purposes. The agent was synchronous with a frequency of $20 \mathrm{~Hz}$, the input of the environment interface was implemented as asynchronous (it is triggered by any sensory input, such as camera images and camera gimbal states). However, the output of the environment interface (i.e., the state and reward) is generated every time an action from the agent is received. Despite the fact that the system architecture was implemented to be nonspecific, as described in Section 4.3.2, there existed small differences between simulated and real experiment setups.

\subsubsection{Simulation}

The simulated environment, designed for training the reinforcement-learning agent, was generated under the Gazebo (http://gazebosim.org) simulator and RotorS UAV simulator [57]. An AscTec Hummingbird multirotor was selected as the RL-M, due to its similarities with the real-flight platform. A generic NC-M was included in the simulation, based on which the ground-truth RoI was generated. An Nvidia GeForce GTX 1070 was used to train and test the reinforcement-learning simulated agent. A near replica of the real-flight software camera gimbal was implemented in simulation.

\subsubsection{Real Flight}

The Parrot Bebop 2 was chosen as the real-flight multirotor platform for both NC-M and follower RL-M due to its frictionless integration, stability, size and manoeuvrability (see Figure 1). The motion-control policy and multirotor detector were successfully tested on an average CPU and GPU (at least 1 GB of video memory). The Parrot Bebop 2 camera gimbal has a resolution of $856 \times 480$, a horizontal FOV of $1.3463 \mathrm{rad}$, a maximum camera gimbal velocity of $0.22 \mathrm{rad} / \mathrm{s}$ and maximum camera 
gimbal angles of 0.87 and $0.61 \mathrm{rad}$ in the $\mathrm{x}$ and $\mathrm{y}$ axes, respectively. Although the reinforcement-learning agent was exactly trained as stated in Section 4.3.1, that is, controlled in attitude for $\mathrm{x}$ and $\mathrm{y}$ axes and altitude velocity for $\mathrm{z}$ axis, it was slightly different for the real-flight platform. Parrot Bebop 2 does not allow for pure attitude control, so it was controlled in velocity (in the three axes of space). The adaptation for the commanded actions is

$$
\begin{aligned}
& v_{x}=k \theta \\
& v_{y}=k \phi,
\end{aligned}
$$

where $k$ was experimentally set and represents the adaptation coefficient $\left(0.23 \mathrm{~m} \mathrm{rad}^{-1} \mathrm{~s}^{-1}\right) ; v_{x}$ and $v_{y}$ represent velocity in the $\mathrm{x}$ and $\mathrm{y}$ axes, respectively; and $\theta$ and $\phi$ are commanded pitch and roll, respectively. This adaptation is only valid for stationary values of the pitch and roll angles and contributes to the differences between the simulated and real multirotor platform.

\subsection{Training Methodology}

\subsubsection{Multirotor Detector}

The multirotor detector was trained with standard supervised-learning techniques by means of synthetic images. These synthetic images were generated under the photorealistic Unreal (https://www.unrealengine.com) game engine and also released as an open-source dataset (see Supplementary Material). For our approach to be successful with our synthetic dataset, the detector network was pretrained on the COCO dataset [58] and fine-tuned with our syntethic dataset (new synthetic multirotor class not available in COCO dataset). Our dataset was mainly composed of two synthetic multirotor models (Parrot ArDrone and DJI Matrice 100) and a small subset of birds and cars extracted from the COCO dataset. We found that, with the addition of these two extra classes, we were able to decrease the total number of false positives. A small multirotor-validation dataset composed of real images was also annotated in order to select the most appropriate network and to provide training and validation results. The training and validation datasets were composed of approximately 8000 and 300 samples, respectively. A reduced set of samples from the training and validation datasets is depicted in Figure 4.

The whole network was trained with the Adam optimizer, with a minibatch size of 1 and the remaining hyperparameters as in Reference [47]. We fine-tuned our network for six epochs (10,000 update steps each with frozen backbone weights) and selected the best one in terms of mean Average Precision (AP) performance. In Table 1, AP is shown for every epoch of training and validation and taking into consideration in a separate trend both the full dataset and the part of the dataset corresponding to the multirotor class. Additionally, a special case was included, where the network was not yet trained with synthetic images (epoch 0). In this special scenario, since the multirotor class was not present in the COCO dataset, the airplane class was selected instead (stated class has been the closest in terms of $\mathrm{AP}$, among others). The average inference time of the network has been $100 \mathrm{~ms}$ (Nvidia GeForce GTX 950M). Nevertheless, in order to assure a RoI in every frame during the whole experiment, as explained in previous sections, a state-of-the-art tracker was also integrated. The multirotor detector was periodically executed during the experiment in order to refine the tracker RoI. Backbone weight freezing with final subnetwork fine tuning were crucial for the detector to generalize for real-domain multirotor images based on pure synthetic multirotor-training images. Complete model retraining did not provide competent results for the application under study. Hence, the backbone extracted a high-dimensional feature map that was rich enough to extract an encoding of the input image, which was latterly classified by the final subnetworks to decide the corresponding class. 

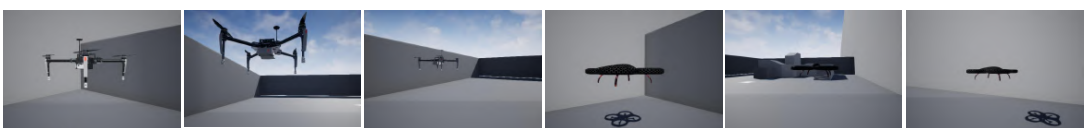

(a)
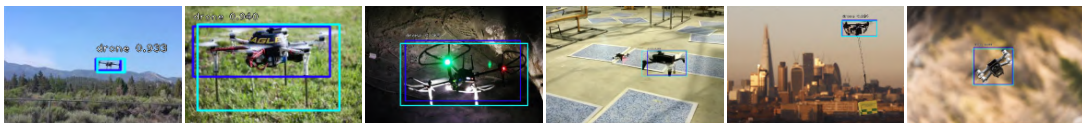

(b)
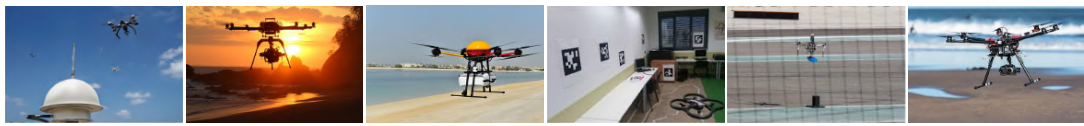

(c)
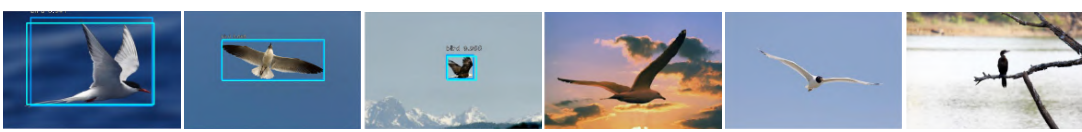

(d)
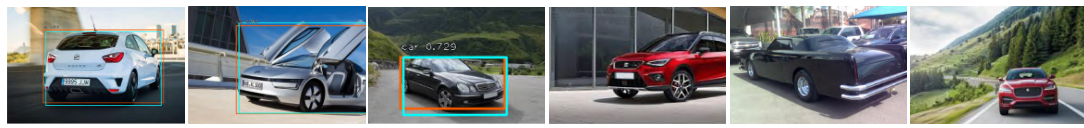

(e)

Figure 4. Samples from multirotor-detector results (ground-truth Region of Interest (RoI), pale blue; detected RoI, dark blue). (a) Training samples from synthetic dataset. (b) Validation samples from multirotor dataset (true positive). (c) Validation samples from multirotor dataset (false negative). (d) Validation samples from birds dataset (true positive and false negative). (e) Validation samples from cars dataset (true positive and false negative).

Table 1. Average Precision (AP) was computed for a minimum Intersection over Union (IoU) of [0.5:0.05:0.95], 0.5 and 0.75 ( $\mathrm{AP}, \mathrm{AP}_{50}$ and $\mathrm{AP}_{75}$, respectively). Network of epoch 0 corresponds to a special case $\left(0^{*}\right)$, where network was not yet trained with synthetic images (only with real images of nonmultirotor classes from COCO - Common Objects in Context - dataset) and was listed in order to make more salient the effect of our synthetic dataset. Network of epoch 4 (bold) was selected for experimentation due to its capability of generalization.

\begin{tabular}{ccccccccccccc}
\hline & & $\begin{array}{c}\text { Training } \\
\text { (only NC-M) }\end{array}$ & & \multicolumn{3}{c}{$\begin{array}{c}\text { Validation } \\
\text { (only NC-M) }\end{array}$} & & \multicolumn{2}{c}{$\begin{array}{c}\text { Training } \\
\text { (full) }\end{array}$} & & \multicolumn{2}{c}{$\begin{array}{c}\text { Validation } \\
\text { (full) }\end{array}$} \\
\hline Epoch & $\mathbf{A P}$ & $\mathbf{A P}_{\mathbf{5 0}}$ & $\mathbf{A P}_{\mathbf{7 5}}$ & $\mathbf{A P}$ & $\mathbf{A P}_{\mathbf{5 0}}$ & $\mathbf{A P}_{\mathbf{7 5}}$ & $\mathbf{A P}$ & $\mathbf{A P}_{\mathbf{5 0}}$ & $\mathbf{A P}_{\mathbf{7 5}}$ & $\mathbf{A P}$ & $\mathbf{A P}_{\mathbf{5 0}}$ & $\mathbf{A P}_{\mathbf{7 5}}$ \\
\hline $0^{*}$ & 0.73 & 0.98 & 0.54 & 0.24 & 0.35 & 0.30 & 0.30 & 0.58 & 0.54 & 0.59 & 0.73 \\
1 & 0.91 & 0.99 & 0.99 & 0.25 & 0.35 & 0.31 & 0.69 & 0.83 & 0.76 & 0.73 & 0.96 & 0.69 \\
2 & 0.92 & 0.99 & 0.99 & 0.63 & 0.93 & 0.70 & 0.76 & 0.88 & 0.83 & 0.75 & 0.96 & 0.87 \\
3 & 0.94 & 0.99 & 0.99 & 0.61 & 0.92 & 0.70 & 0.76 & 0.88 & 0.83 & 0.73 & 0.95 & 0.85 \\
4 & $\mathbf{0 . 9 3}$ & $\mathbf{0 . 9 9}$ & $\mathbf{0 . 9 9}$ & $\mathbf{0 . 6 4}$ & $\mathbf{0 . 9 3}$ & $\mathbf{0 . 7 5}$ & $\mathbf{0 . 8 5}$ & $\mathbf{0 . 9 5}$ & $\mathbf{0 . 9 2}$ & $\mathbf{0 . 7 5}$ & $\mathbf{0 . 9 7}$ & $\mathbf{0 . 8 9}$ \\
5 & 0.95 & 0.99 & 0.99 & 0.61 & 0.89 & 0.69 & 0.95 & 0.99 & 0.98 & 0.74 & 0.95 & 0.87 \\
6 & 0.95 & 0.99 & 0.99 & 0.60 & 0.89 & 0.68 & 0.95 & 0.98 & 0.98 & 0.73 & 0.96 & 0.88 \\
\hline
\end{tabular}

\subsubsection{Motion-Control Policy}

The reinforcement-learning problem was treated as episodic, that is, once the agent reached an absorbing state, the episode reset and both multirotors were respawned to a uniformly randomized position within the RL-M FOV boundaries. The states where the agent perceived $r_{1}$ or $r_{2}$ as its 
instantaneous reward were considered absorbing states (refer to Equation (7)). The time length of an episode is limited to 1000 time steps (considering a time step of $0.05 \mathrm{~s}$, as previously stated). The NC-M RoI was synthetically generated for training the reinforcement-learning agent by means of the 3D ground-truth position of the NC-M and the simulated camera gimbal intrinsic parameters. However, it showed similar performance when tested with the RoI generated by our multirotor detector and tracker. The NC-M was also completely static during the whole training process. This fact did not deteriorate the final performance since the distribution of states and actions experimented with either static or moving NC-M was equivalent and the actor-critic network did not take temporal structure into consideration (feed-forward neural network).

As shown in Figure 5, in order to provide a baseline for training, DDPG has been included in the study. Hence, both DDPG and PPO algorithms has been trained in the same conditions. Nevertheless, DDPG has not provided adequate results in terms of practical performance, even when the average of the accumulated reward seems to reach similar values with respect to PPO. Considering these results, only PPO has been selected for further experimentation (refer to Section 6 for more in-depth details). Both DDPG and PPO actor-critic networks have been trained with Adam optimizer, with a learning rate of $3 \times 10^{-4}$ and a minibatch size of 64 . DDPG has been trained with a soft target update rate $\tau$ of 0.001 [51]. PPO has been trained with a policy clipping value of 0.1 [53]. The agent has been trained over 4432 update steps (with trajectories of 256 experiences) for a total time of approximately $48 \mathrm{~h}$ and $16 \mathrm{~h}$ for DDPG and PPO, respectively. Each weights update step incurs in 8 epochs with the stated minibatch size. In the case of PPO, which has been the only algorithm to provide the full desired behavior, several networks have been tested from update step 3000 to update step 4300, being able to select actor-critic network of update step 4220, which has shown the best results in terms of performance for the scenarios presented in this work. Stated performance was measured with a small subset of 10 testing trials for every network pre-selected and utilizing metrics shown in Table 2. After performance-based pre-selection, the network of episode 4220 has been exhaustively tested for both simulated and real-flights.

Table 2. RoI center and area error statistics for the whole set of experiments (simulated and real-flight cases). Average, maximum and minimum values are provided.

\begin{tabular}{ccccccc}
\hline & \multicolumn{3}{c}{ Simulation } & \multicolumn{3}{c}{ Real Flights } \\
\hline $\begin{array}{c}\text { Experiment } \\
\text { scenario }\end{array}$ & \multicolumn{3}{c}{ Center error (px) } & \multicolumn{3}{c}{ Center error (px) } \\
& Avg & Max & Min & Avg & Max & Min \\
\hline X-axis & 5.79 & 24 & 0 & 15.15 & 44 & 0 \\
Y-axis & 5.72 & 27 & 0 & 22.35 & 80 & 0 \\
Z-axis & 7.58 & 39 & 0 & 20.31 & 66 & 0 \\
Arbitrary & 5.7 & 24 & 0 & 26.71 & 65 & 0 \\
\hline Experiment & \multicolumn{3}{c}{ Area error $(\%)$} & \multicolumn{3}{c}{ Area error $(\%)$} \\
scenario & Avg & Max & Min & Avg & Max & Min \\
\hline X-axis & 13.61 & 52.51 & 0.16 & 33.59 & 57.38 & 0 \\
Y-axis & 8.59 & 24.10 & 0.16 & 44.02 & 148 & 0 \\
Z-axis & 17.41 & 74.51 & 0 & 47.12 & 104 & 0 \\
Arbitrary & 10.94 & 47.56 & 0 & 63.99 & 162.8 & 0.6 \\
\hline
\end{tabular}

\subsection{Simulated and Real-Flight Experiments}

A representative set of experiments that were able to illustrate the complete performance of our approach for both simulated and real flights were designed. Furthermore, with the aim of testing the performance of our approach in every axis of space, a separate X-axis, $Y$-axis and Z-axis experiment was carried out. In order to provide complete results about the final behavior and to bring up possible coupling interferences between axes, we performed an arbitrary experiment (refer to Figures 6 and 7). Every experiment was carried out under maximum velocity conditions (that allowed RL-M to keep track of the NC-M), which were extracted from experimentation. For every performed experiment, 2D and 3D ground-truth trajectories, as well as current-target RoI center error (in pixels) and current-target RoI area error are depicted. For a complete overview of the experiment, refer to the Supplementary Material section. 


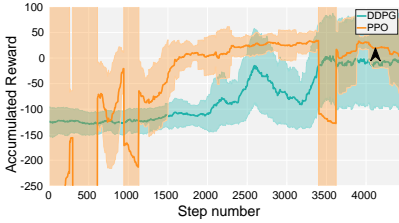

(a)

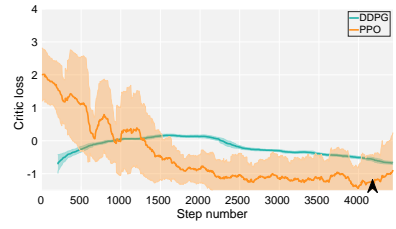

(b)

Figure 5. Moving average and standard deviation over 50 update steps of the training. (a) Accumulated Reward and (b) Critic loss function (logarithmic representation). The network corresponding to update step 4220 provided the best performance.

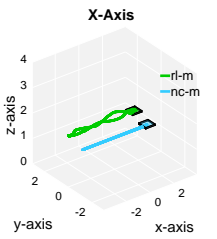

(a)

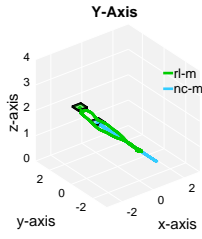

(e)

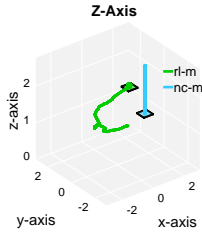

(i)

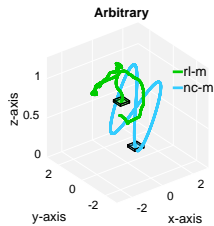

(m)

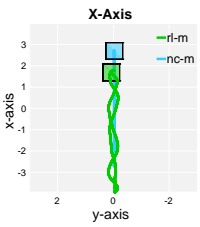

(b)

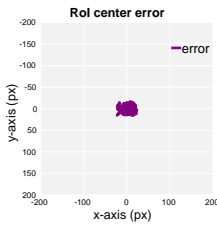

(c)

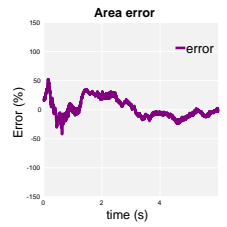

(d)

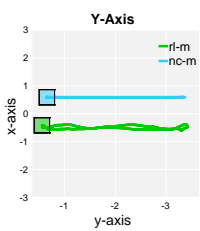

(f)

Z-Axis

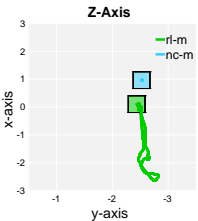

(j)

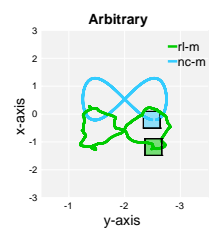

(n)

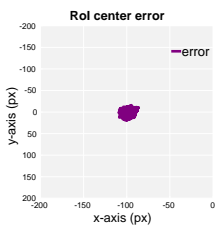

(g)

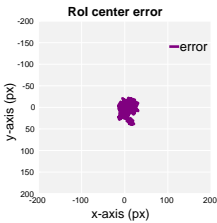

(k)

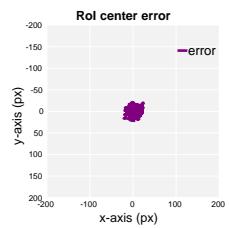

(o)

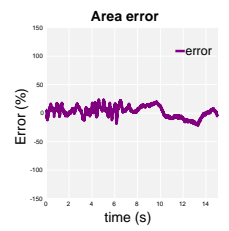

(h)

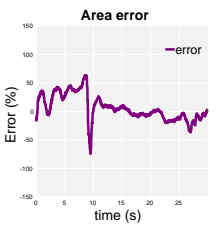

(1)

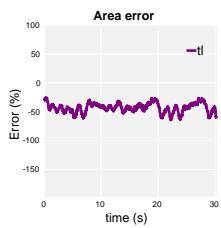

(p)

Figure 6. Simulation results corresponding to four different scenarios for our multirotor following approach. $(\mathbf{a}, \mathbf{b}, \mathbf{e}, \mathbf{f})$ Rectilinear periodic trajectory of the Non-Cooperative Multirotor (NC-M) along the 
$\mathrm{x}$ and $\mathrm{y}$ axes with a maximum velocity of $1.3 \mathrm{~m} / \mathrm{s}$ (X-Axis and $Y$-Axis, respectively). (i,j) Rectilinear periodic trajectory of the NC-M along $\mathrm{z}$ axis with a maximum velocity of $0.5 \mathrm{~m} / \mathrm{s}$ (Z-Axis). $(\mathbf{m}, \mathbf{n})$ Arbitrary non-periodic trajectory of the NC-M with a maximum velocity of $0.47 \mathrm{~m} / \mathrm{s}$ (Arbitrary). $(\mathbf{c}, \mathbf{d}, \mathbf{g}, \mathbf{h}, \mathbf{k}, \mathbf{l}, \mathbf{o}, \mathbf{p})$ Current-target RoI center and area error corresponding to each of the four stated experiments. Multirotor trajectories have been generated by a groundtruth source.

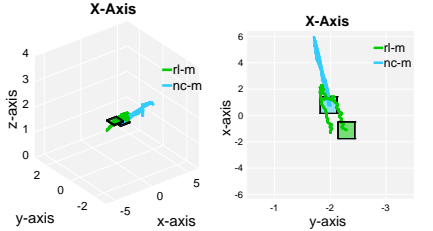

(a)

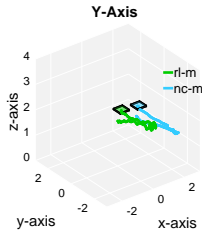

(e)

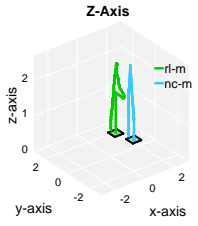

(i)

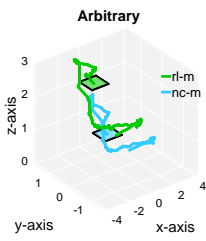

(m) (b)

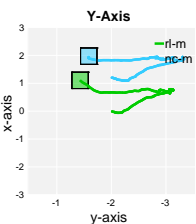

(f)

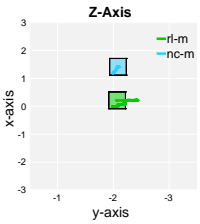

(j)

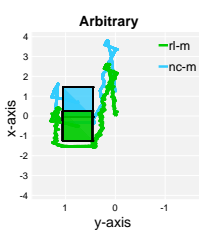

(n)

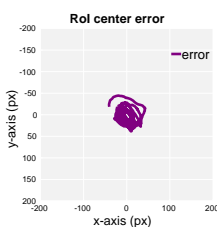

(c)

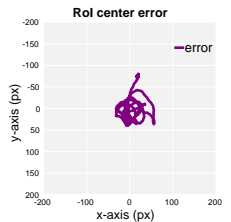

(g)

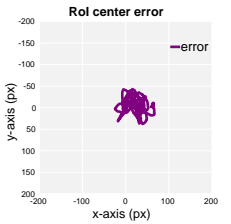

(k)

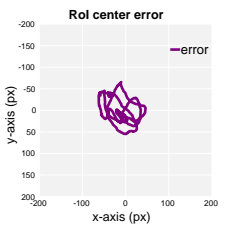

(o)

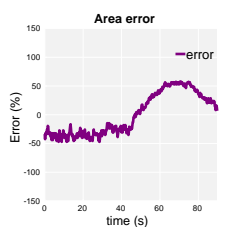

(d)

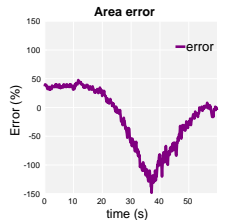

(h)

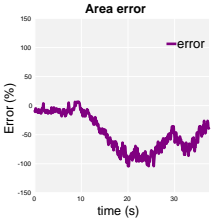

(1)

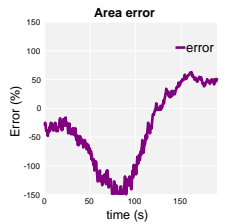

(p)

Figure 7. Real-flight results corresponding to four different scenarios for our multirotor following approach. (a,b,e, $\mathbf{f}, \mathbf{i}, \mathbf{j})$ Rectilinear periodic trajectory of Noncooperative Multirotor (NC-M) along $\mathrm{x}, \mathrm{y}$ and $\mathrm{z}$ axes with a maximum velocity of $0.3 \mathrm{~m} / \mathrm{s}$ (X-Axis, Y-Axis and Z-Axis, respectively). $(\mathbf{m}, \mathbf{n})$ Arbitrary nonperiodic trajectory of NC-M with maximum velocity of $0.3 \mathrm{~m} / \mathrm{s}$ (Arbitrary). $(\mathbf{c}, \mathbf{d}, \mathbf{g}, \mathbf{h}, \mathbf{k}, \mathbf{l}, \mathbf{o}, \mathbf{p})$ Current-target $\mathrm{RoI}$ center and area error corresponding to each of four stated experiments. Multirotor trajectories have been generated by an Extended Kalman Filter (EKF) that utilizes optical-flow velocities and measured accelerations as only input source.

\section{Discussion}

The considerable testing and validation of the presented multirotor following technique describes a proper pipeline to utilize synthetic data in the context of a challenging real-world and image-based 
application. It was tested in both simulated and real-flight environments in order to demonstrate the approach and to compare the differences between scenarios. On the one hand, the multirotor detector showed outstanding performance with untrained real multirotor images. It sufficiently generalized not only for the multirotor used in this work but for a wide variety of multirotors of the validation dataset (see Table 1). Epoch 0 of training showed inadequate network performance, while the following epochs showed an abrupt growth of the AP for both partial and full datasets. Results validated our technique and confirmed the benefits of the inclusion of our synthetic dataset. Additionally, this trend enables numerous applications based on multirotor detection and drafts a training pipeline for unavailable annotated datasets, such as the case of this study.

On the other hand, the multirotor motion-control policy, which was only trained in a simulated environment with NC-M in random and static positions, sufficiently generalized to perform in simulated scenarios where the NC-M was dynamically moving, as well as in real-flight scenarios with similar conditions. To the best of the authors' knowledge, it was the first time that the camera gimbal motion was jointly controlled with multirotor motion based on image cues. Additionally, an approach where complex and image-based behavior was trained in a synthetic environment and used in the real world was validated.

The simulated and real-world environments were similar but not perfect, such as the FC response or the multirotor dynamics. Indeed, we found a decrease in performance of the approach in real-flight experiments compared with the simulated scenarios (refer to maximum velocities in Figures 6 and 7). This can be due to differences between real and simulated multirotor dynamics, FC response and the camera gimbal model. Nevertheless, even with a decrease in performance, the transition of the approach from simulation to real world was direct, which was the overall aim of this work, taking into consideration the stated adaptation from Section 5.1.2.

Even though natural policy methods show outstanding data efficiency when compared with previous methods, such as the DDPG method, there are still sample inefficiencies that are a matter of community research (4432 update steps over $16 \mathrm{~h}$ ). However, for the PPO method, both accumulated reward performance substantially improved after a few hours of training (see Figure 5). DDPG managed to improve its performance in terms of accumulated reward but, compared to the PPO method, it resulted in higher variance, a lower average of accumulated reward and longer training times (approximately $48 \mathrm{~h}$ ). It also did not provide practical results (the variance of the agent actions was very low and usage of the camera gimbal was not present).

Regarding Figure 6, our approach showed outstanding performance in all experiment scenarios. The multirotor was able to keep track of the NC-M during the whole set of tests, being able to follow the target at a maximum velocity of $1.3 \mathrm{~m} / \mathrm{s}$ for the X-Axis and $Y$-Axis experiments. As seen in Figure 7 , the agent was able to show a similar behavior compared to the simulated environments, following the NC-M at a maximum velocity of $0.3 \mathrm{~m} / \mathrm{s}$ for the arbitrary experiment. Due to differences in the simulated and real-flight autopilot capabilities, tracking the NC-M for Z-Axis experiment was best achieved through camera gimbal actions for simulation and through RL-M altitude velocity actions for the real-flight experiments (although keeping the NC-M within the camera FOV in both experiments).

In Table 2, we can see that both the simulated and real-flight results were in a similar order of magnitude and showed suitable behavior (the RoI was being centered during the whole experiment scenario and, in a second order of priority, the area was being compensated). The processing overhead of both multirotor detector and motion-control policy was reduced and could be executed in an average CPU (motion-control policy) and GPU (multirotor detector).

\section{Conclusions and Future Work}

In this paper, the vision-based following of a noncooperative multirotor was presented. The approach was mainly based on synthetic data and utilized deep- and reinforcement-learning techniques for the multirotor detector and motion-control policy, respectively. The multirotor detector was trained with synthetic images generated by a commercial game engine and was able to generalize 
to a wide range of real-world multirotors and environments. The camera gimbal and multirotor motion control were jointly treated within a motion-control policy, fully learned in a simulated multirotor environment. The complete approach was tested in both simulated and real-flight environments providing proper results, even in the presence of virtual-to-real differences in the multirotor and camera-gimbal models.

This study could be extended by improving the performance of both the multirotor detector and the motion-control policy. On the one hand, to improve the multirotor detector, the synthetic dataset could be extended and other network structures could be tested (e.g., MobileNet-SSD and YOLO). On the other hand, in order to improve the motion-control policy, both the simulated multirotor and the camera gimbal could be more realistically modeled and end-to-end fully convolutional network structure could be included as a future line of research.

Supplementary Materials: The following are available online at http:/ / www.mdpi.com/1424-8220/19/21/4794/ s1.

Author Contributions: The contribution of each author to the article has been: conceptualization, A.R.-R.; methodology, A.R.-R. and A.A.-F.; software, A.R.-R., A.A.-F. and H.B.; validation, A.R.-R. and A.A.-F.; formal analysis, A.R.-R.; investigation, A.R.-R. and A.A.-F.; data curation, A.R.-R.; writing-original draft preparation, A.R.-R. and A.A.-F.; writing-review and editing, A.R.-R., A.A.-F. and H.B.; visualization, A.R.-R. and A.A.-F.; resources, P.C. and J.P.H.; general supervision, P.C. and J.P.H.; project management, P.C. and J.P.H.; funding procurement, P.C. and J.P.H.

Funding: This research was partially funded by the "Drone Autonomy" project with the Aerospace Controls Laboratory at MIT, headed by Prof. Jonathan How, funded by MISTI: MITInternational Science and Technology Initiatives (project period: 1 January 2018-31 August 2019), and "COMplex Coordinated Inspection and Security missions by UAVs in cooperation with UGV", funded by the Spanish Ministry of Economy and Competition (RTI2018-100847-B-C21). Start date: 2019; end date: 2021.

Acknowledgments: Thanks to Carlos Sampedro and Joaquin Zafra for their inestimable advice and help during experimentation, respectively.

Conflicts of Interest: The authors declare no conflict of interest.

\section{Abbreviations}

The following abbreviations are used in this manuscript:

$\begin{array}{ll}\text { UPM } & \text { Universidad Politécnica de Madrid } \\ \text { CSIC } & \text { Consejo Superior de Investigaciones Científicas } \\ \text { DOF } & \text { Degrees Of Freedom } \\ \text { FC } & \text { Flight Controller } \\ \text { RGB } & \text { Red Green Blue } \\ \text { UAV } & \text { Unmanned Aerial Vehicle } \\ \text { PID } & \text { Proportional Integral Derivative } \\ \text { RoI } & \text { Region of Interest } \\ \text { HOG } & \text { Histogram Of Gradients } \\ \text { LBP } & \text { Local Binary Patterns } \\ \text { CAD } & \text { Computer-Aided Design } \\ \text { CNN } & \text { Convolutional Neural Network } \\ \text { NC-M } & \text { Noncooperative Multirotor } \\ \text { RL-M } & \text { Reinforcement-Learning-based Multirotor } \\ \text { FPN } & \text { Feature Pyramid Network } \\ \text { FL } & \text { Focal Loss } \\ \text { DCF } & \text { Discriminative Correlation Filter } \\ \text { DDPG } & \text { Deep Deterministic Policy Gradients } \\ \text { TRPO } & \text { Trust-Region Policy Optimization } \\ \text { PPO } & \text { Proximal Policy Optimization } \\ \text { FOV } & \text { Field Of View } \\ \text { ROS } & \text { Robotic Operating System } \\ \text { COCO } & \text { Common Objects in COntext } \\ & \\ \end{array}$




\section{References}

1. Tan, J.; Zhang, T.; Coumans, E.; Iscen, A.; Bai, Y.; Hafner, D.; Bohez, S.; Vanhoucke, V. Sim-to-real: Learning agile locomotion for quadruped robots. arXiv 2018, arXiv:1804.10332.

2. Rodriguez-Ramos, A.; Sampedro, C.; Bavle, H.; Moreno, I.G.; Campoy, P. A Deep Reinforcement Learning Technique for Vision-Based Autonomous Multirotor Landing on a Moving Platform. In Proceedings of the 2018 IEEE/RSJ International Conference on Intelligent Robots and Systems (IROS), Madrid, Spain, 1-5 October 2018; pp. 1010-1017.

3. Hwangbo, J.; Sa, I.; Siegwart, R.; Hutter, M. Control of a quadrotor with reinforcement learning. IEEE Robot. Autom. Lett. 2017, 2, 2096-2103. [CrossRef]

4. Rozantsev, A.; Lepetit, V.; Fua, P. On rendering synthetic images for training an object detector. Comput. Vis. Image Underst. 2015, 137, 24-37. [CrossRef]

5. Tobin, J.; Fong, R.; Ray, A.; Schneider, J.; Zaremba, W.; Abbeel, P. Domain randomization for transferring deep neural networks from simulation to the real world. In Proceedings of the 2017 IEEE/RSJ International Conference on Intelligent Robots and Systems (IROS), Vancouver, BC, Canada, 24-28 September 2017.

6. Chen, Y.; Li, W.; Sakaridis, C.; Dai, D.; Van Gool, L. Domain adaptive faster r-cnn for object detection in the wild. In Proceedings of the IEEE Conference on Computer Vision and Pattern Recognition, Salt Lake, UT, USA, 18-22 June 2018; pp. 3339-3348.

7. LeCun, Y.; Bengio, Y.; Hinton, G. Deep learning. Nature 2015, 521, 436. [CrossRef] [PubMed]

8. Mnih, V.; Kavukcuoglu, K.; Silver, D.; Rusu, A.A.; Veness, J.; Bellemare, M.G.; Graves, A.; Riedmiller, M.; Fidjeland, A.K.; Ostrovski, G.; et al. Human-level control through deep reinforcement learning. Nature 2015, 518, 529. [CrossRef] [PubMed]

9. Zhang, J.; Tai, L.; Yun, P.; Xiong, Y.; Liu, M.; Boedecker, J.; Burgard, W. Vr-goggles for robots: Real-to-sim domain adaptation for visual control. IEEE Robot. Autom. Lett. 2019, 4, 1148-1155. [CrossRef]

10. Rozantsev, A.; Lepetit, V.; Fua, P. Detecting flying objects using a single moving camera. IEEE Trans. Pattern Anal. Mach. Intell. 2016, 39, 879-892. [CrossRef]

11. Li, J.; Ye, D.H.; Chung, T.; Kolsch, M.; Wachs, J.; Bouman, C. Multi-target detection and tracking from a single camera in Unmanned Aerial Vehicles (UAVs). In Proceedings of the 2016 IEEE/RSJ International Conference on Intelligent Robots and Systems (IROS), Deajeon, Korea, 9-14 October 2016; pp. 4992-4997.

12. Opromolla, R.; Fasano, G.; Accardo, D. A Vision-Based Approach to UAV Detection and Tracking in Cooperative Applications. Sensors 2018, 18, 3391. [CrossRef]

13. Zengin, U.; Dogan, A. Cooperative target pursuit by multiple UAVs in an adversarial environment. Robot. Autonom. Syst. 2011, 59, 1049-1059. [CrossRef]

14. Yamasaki, T.; Balakrishnan, S. Sliding mode based pure pursuit guidance for UAV rendezvous and chase with a cooperative aircraft. In Proceedings of the 2010 American Control Conference, Baltimore, MD, USA, 30 June-2 July 2010.

15. Alexopoulos, A.; Schmidt, T.; Badreddin, E. Cooperative pursue in pursuit-evasion games with unmanned aerial vehicles. In Proceedings of the 2015 IEEE/RSJ International Conference on Intelligent Robots and Systems (IROS), Hamburg, Germany, 28 September-3 October 2015.

16. Ma, L.; Hovakimyan, N. Vision-based cyclic pursuit for cooperative target tracking. J. Guid. Control Dyn. 2013, 36, 617-622. [CrossRef]

17. Yamasaki, T.; Enomoto, K.; Takano, H.; Baba, Y.; Balakrishnan, S. Advanced pure pursuit guidance via sliding mode approach for chase UAV. In Proceedings of the AIAA Guidance, Navigation, and Control Conference, Chicago, IL, USA, 10 August-13 August 2009.

18. Pestana, J.; Sanchez-Lopez, J.; Saripalli, S.; Campoy, P. Computer vision based general object following for gps-denied multirotor unmanned vehicles. In Proceedings of the 2014 American Control Conference, Portland, OR, USA, 4-6 June 2014.

19. Teuliere, C.; Eck, L.; Marchand, E. Chasing a moving target from a flying UAV. In Proceedings of the 2011 IEEE/RSJ International Conference on Intelligent Robots and Systems, San Francisco, CA, USA, 25-30 September 2011. 
20. Choi, H.; Kim, Y. UAV guidance using a monocular-vision sensor for aerial target tracking. Control Eng. Pract. 2014, 22, 10-19. [CrossRef]

21. Li, R.; Pang, M.; Zhao, C.; Zhou, G.; Fang, L. Monocular long-term target following on uavs. In Proceedings of the IEEE Conference on Computer Vision and Pattern Recognition Workshops, Las Vegas, NV, USA, 26 June-1 July 2016; pp. 29-37.

22. Mueller, M.; Sharma, G.; Smith, N.; Ghanem, B. Persistent aerial tracking system for uavs. In Proceedings of the 2016 IEEE/RSJ International Conference on Intelligent Robots and Systems (IROS), Deajeon, Korea, 9-14 October 2016; pp. 1562-1569.

23. Kalal, Z.; Mikolajczyk, K.; Matas, J. Tracking-learning-detection. Pattern Anal. Mach. Intell. 2011, 34, 1409-1422. [CrossRef] [PubMed]

24. Hare, S.; Golodetz, S.; Saffari, A.; Vineet, V.; Cheng, M.M.; Hicks, S.L.; Torr, P.H. Struck: Structured output tracking with kernels. IEEE Trans. Pattern Anal. Mach. Intell. 2015, 38, 2096-2109. [CrossRef] [PubMed]

25. Kassab, M.A.; Maher, A.; Elkazzaz, F.; Baochang, Z. UAV Target Tracking By Detection via Deep Neural Networks. In Proceedings of the 2019 IEEE International Conference on Multimedia and Expo (ICME), Shanghai, China, 8-12 July 2019; pp. 139-144.

26. Maher, A.; Taha, H.; Zhang, B. Realtime multi-aircraft tracking in aerial scene with deep orientation network. J. Real-Time Image Process. 2018, 15, 495-507. [CrossRef]

27. Yao, N.; Anaya, E.; Tao, Q.; Cho, S.; Zheng, H.; Zhang, F. Monocular vision-based human following on miniature robotic blimp. In Proceedings of the 2017 IEEE International Conference on Robotics and Automation (ICRA), Singapore, 29 May-3 June 2017; pp. 3244-3249.

28. Nägeli, T.; Oberholzer, S.; Plüss, S.; Alonso-Mora, J.; Hilliges, O. Flycon: Real-Time EnvironmentIndependent Multi-View Human Pose Estimation with Aerial Vehicles. In Proceedings of the SIGGRAPH Asia 2018 Technical, Tokio, Japan, 4-7 December 2018; p. 182.

29. Rafi, F.; Khan, S.; Shafiq, K.; Shah, M. Autonomous target following by unmanned aerial vehicles. In Proceedings of the Unmanned Systems Technology VIII. International Society for Optics and Photonics, San Jose, CA, USA, 20-21 January 2006.

30. Qadir, A.; Neubert, J.; Semke, W.; Schultz, R. On-Board Visual Trackingwith Unmanned Aircraft System (UAS); American Institute of Aeronautics and Astronautics: Reston, VA, USA, 2011.

31. Andrychowicz, M.; Baker, B.; Chociej, M.; Jozefowicz, R.; McGrew, B.; Pachocki, J.; Petron, A.; Plappert, M.; Powell, G.; Ray, A.; et al. Learning Dexterous in-Hand Manipulation. arXiv 2018, arXiv:1808.00177.

32. James, S.; Wohlhart, P.; Kalakrishnan, M.; Kalashnikov, D.; Irpan, A.; Ibarz, J.; Levine, S.; Hadsell, R.; Bousmalis, K. Sim-to-Real via Sim-to-Sim: Data-efficient Robotic Grasping via Randomized-to-Canonical Adaptation Networks. arXiv 2018, arXiv:1812.07252.

33. Sampedro, C.; Rodriguez-Ramos, A.; Gil, I.; Mejias, L.; Campoy, P. Image-Based Visual Servoing Controller for Multirotor Aerial Robots Using Deep Reinforcement Learning. In Proceedings of the 2018 IEEE/RSJ International Conference on Intelligent Robots and Systems (IROS), Madrid, Spain, 1-5 October 2018; pp. 979-986.

34. Kang, K.; Belkhale, S.; Kahn, G.; Abbeel, P.; Levine, S. Generalization through simulation: Integrating simulated and real data into deep reinforcement learning for vision-based autonomous flight. arXiv 2019, arXiv:1902.03701.

35. Sadeghi, F.; Levine, S. CAD2RL: Real single-image flight without a single real image. arXiv 2016, arXiv:1611.04201.

36. Wang, F.; Zhou, B.; Chen, K.; Fan, T.; Zhang, X.; Li, J.; Tian, H.; Pan, J. Intervention Aided Reinforcement Learning for Safe and Practical Policy Optimization in Navigation. arXiv 2018, arXiv:1811.06187.

37. Gökçe, F.; Üçoluk, G.; Şahin, E.; Kalkan, S. Vision-based detection and distance estimation of micro unmanned aerial vehicles. Sensors 2015, 15, 23805-23846. [CrossRef]

38. Fu, C.; Duan, R.; Kircali, D.; Kayacan, E. Onboard robust visual tracking for UAVs using a reliable global-local object model. Sensors 2016, 16, 1406. [CrossRef] [PubMed]

39. Hinterstoisser, S.; Lepetit, V.; Wohlhart, P.; Konolige, K. On pre-trained image features and synthetic images for deep learning. In Proceedings of the European Conference on Computer Vision (ECCV), Munich, Germany, 8-14 September 2018.

40. Peng, X.; Sun, B.; Ali, K.; Saenko, K. Learning deep object detectors from 3d models. In Proceedings of the IEEE International Conference on Computer Vision, Las Condes, Chile, 11-18 December 2015. 
41. Shrivastava, A.; Pfister, T.; Tuzel, O.; Susskind, J.; Wang, W.; Webb, R. Learning from simulated and unsupervised images through adversarial training. In Proceedings of the IEEE Conference on Computer Vision and Pattern Recognition, Honolulu, HI, USA, 21-26 July 2017.

42. Tremblay, J.; Prakash, A.; Acuna, D.; Brophy, M.; Jampani, V.; Anil, C.; To, T.; Cameracci, E.; Boochoon, S.; Birchfield, S. Training deep networks with synthetic data: Bridging the reality gap by domain randomization. In Proceedings of the IEEE Conference on Computer Vision and Pattern Recognition Workshops, Salt Lake City, UT, USA, 18-23 June 2018; pp. 969-977.

43. Ren, S.; He, K.; Girshick, R.; Sun, J. Faster r-cnn: Towards real-time object detection with region proposal networks. In Proceedings of the Advances in Neural Information Processing Systems, Montreal, QC, Canada, 7-12 December 2015; pp. 91-99.

44. Dai, J.; Li, Y.; He, K.; Sun, J. R-fon: Object detection via region-based fully convolutional networks. In Proceedings of the Advances in Neural Information Processing Systems, Barcelona, Spain, 5-10 December 2016; pp. 379-387.

45. He, K.; Gkioxari, G.; Dollár, P.; Girshick, R. Mask r-cnn. In Proceedings of the IEEE International Conference on Computer Vision, Venezia, Italy, 22-29 October 2017; pp. 2961-2969.

46. Alabachi, S.; Sukthankar, G.; Sukthankar, R. Customizing Object Detectors for Indoor Robots. arXiv 2019, arXiv:1902.10671.

47. Lin, T.; Goyal, P.; Girshick, R.; He, K.; Dollár, P. Focal loss for dense object detection. In Proceedings of the IEEE International Conference on Computer Vision, Venezia, Italy, 22-29 October 2017.

48. He, K.; Zhang, X.; Ren, S.; Sun, J. Deep residual learning for image recognition. In Proceedings of the IEEE Conference on Computer Vision and Pattern Recognition, Las Vegas, NV, USA, 26 June-1 July 2016.

49. Danelljan, M.; Bhat, G.; Shahbaz Khan, F.; Felsberg, M. Eco: Efficient convolution operators for tracking. In Proceedings of the IEEE Conference on Computer Vision and Pattern Recognition, Honolulu, HI, USA, 21-26 July 2017.

50. Sutton, R.S.; Barto, A.G. Reinforcement Learning: An Introduction; MIT Press: Cambridge, MA, USA, 1998.

51. Lillicrap, T.P.; Hunt, J.J.; Pritzel, A.; Heess, N.; Erez, T.; Tassa, Y.; Silver, D.; Wierstra, D. Continuous control with deep reinforcement learning. arXiv 2015, arXiv:1509.02971.

52. Schulman, J.; Levine, S.; Abbeel, P.; Jordan, M.I.; Moritz, P. Trust Region Policy Optimization. In Proceedings of the 31st International Conference on Machine Learning, Beijing, China, 21-26 June 2014.

53. Schulman, J.; Wolski, F.; Dhariwal, P.; Radford, A.; Klimov, O. Proximal policy optimization algorithms. arXiv 2017, arXiv:1707.06347.

54. Andrychowicz, M.; Wolski, F.; Ray, A.; Schneider, J.; Fong, R.; Welinder, P.; McGrew, B.; Tobin, J.; Abbeel, O.P.; Zaremba, W. Hindsight experience replay. In Proceedings of the Advances in Neural Information Processing Systems 2017, Long Beach, CA, USA, 4-9 December 2017; pp. 5048-5058.

55. Dorigo, M.; Colombetti, M. Robot Shaping: An Experiment in Behavior Engineering; MIT Press: Cambridge, MA, USA, 1998.

56. Quigley, M.; Conley, K.; Gerkey, B.; Faust, J.; Foote, T.; Leibs, J.; Wheeler, R.; Ng, A.Y. ROS: An open-source Robot Operating System. In Proceedings of the ICRA Workshop on Open Source Software, Kobe, Japan, 12-17 May 2009.

57. Furrer, F.; Burri, M.; Achtelik, M.; Siegwart, R. Rotors-A modular gazebo mav simulator framework. In Robot Operating System (ROS); Springer: Cham, Switzerland, 2016.

58. Lin, T.Y.; Maire, M.; Belongie, S.; Hays, J.; Perona, P.; Ramanan, D.; Dollár, P.; Zitnick, C.L. Microsoft coco: Common objects in context. In Proceedings of the European Conference on Computer Vision, Zurich, Switzerland, 6-12 September 2014; pp. 740-755.

(C) 2019 by the authors. Licensee MDPI, Basel, Switzerland. This article is an open access article distributed under the terms and conditions of the Creative Commons Attribution (CC BY) license (http://creativecommons.org/licenses/by/4.0/). 


\section{Publication III}

(C) 2020 IEEE. Reprinted, with permission, from:

Rodriguez-Ramos, A., Rodriguez-Vazquez, J., Sampedro, C., and Campoy, P. (2020). Adaptive Inattentional Framework for Video Object Detection With Reward-Conditional Training. IEEE Access, 8, 124451-124466. doi:10.1109/ACCESS.2020.3006191. JCR (2019): 4.670, Computer Science (miscellaneous) (Q1), Engineering (miscellaneous) (Q1). 



\title{
Adaptive Inattentional Framework for Video Object Detection With Reward-Conditional Training
}

\author{
ALEJANDRO RODRIGUEZ-RAMOS ${ }^{\circledR}$, (Graduate Student Member, IEEE), \\ JAVIER RODRIGUEZ-VAZQUEZ, CARLOS SAMPEDRO ${ }^{\circledR}$, (Member, IEEE), \\ AND PASCUAL CAMPOY ${ }^{\circledR}$ (Senior Member, IEEE) \\ Centre for Automation and Robotics, Computer Vision and Aerial Robotics Group, Universidad Politécnica de Madrid (UPM-CSIC), 28006 Madrid, Spain \\ Corresponding author: Alejandro Rodriguez-Ramos (alejandro.rramos@upm.es)
}

This work was supported in part by the Spanish Ministry of Economy and Competitivity through the project (Complex Coordinated Inspection and Security Missions by UAVs in cooperation with UGV) under Grant RTI2018-100847-B-C21, in part by the MIT International Science and Technology Initiatives (MISTI)-Spain through the project (Drone Autonomy), and in part by the Mohamed Bin Zayed International Robotics Challenge (MBZIRC) in the year 2020 (MBZIRC 2020 competition).

\begin{abstract}
Recent object detection studies have been focused on video sequences, mostly due to the increasing demand of industrial applications. Although single-image architectures achieve remarkable results in terms of accuracy, they do not take advantage of particular properties of the video sequences and usually require high parallel computational resources, such as desktop GPUs. In this work, an inattentional framework is proposed, where the object context in video frames is dynamically reused in order to reduce the computation overhead. The context features corresponding to keyframes are fused into a synthetic feature map, which is further refined using temporal aggregation with ConvLSTMs. Furthermore, an inattentional policy has been learned to adaptively balance the accuracy and the amount of context reused. The inattentional policy has been learned under the reinforcement learning paradigm, and using our novel reward-conditional training scheme, which allows for policy training over a whole distribution of reward functions and enables the selection of a unique reward function at inference time. Our framework shows outstanding results on platforms with reduced parallelization capabilities, such as CPUs, achieving an average latency reduction up to $2.09 \times$, and obtaining FPS rates similar to their equivalent GPU platform, at the cost of a $1.11 \times \mathrm{mAP}$ reduction.
\end{abstract}

INDEX TERMS Inattention, YOTO, reward-conditional training, deep learning, video object detection, reinforcement learning, CNN, LSTM, loss-conditional training.

\section{INTRODUCTION}

Recent advances in image object detection have mainly focused on the development of Convolutional Neural Network (CNN) architectures [1]-[4] to progressively increase accuracy or decrease processing times. In this regard, accuracy has been the primary concern in the majority of studies [3], [5]-[7], mostly due to the initial lack of techniques which precisely capture the variability found in the object detection task (number of classes, illumination and environmental ambiences, corner cases, etc.). Nevertheless, detection speed and power consumption are recently becoming

The associate editor coordinating the review of this manuscript and approving it for publication was Ting Wang ${ }^{(\mathbb{D}}$. key differentiator metrics [1], [8]-[13] as deep learning is being increasingly deployed in practical applications, where computing and power resources can be limited. In the context of systems with reduced computational capabilities, such as embedded platforms, substantial effort has been carried out to generate efficient architectures, such as the ones based on SqueezeNet [14], Mobilenet [15]-[18], or ShuffleNet [19], [20]. Also, other studies target efficient representations, such as Binarized Neural Networks (BNNs) [21] or Quantized Neural Networks (QNNs) [22], and low-power hardware implementations [23]-[25]. Despite significant advances in the field, the final objective of processing high-resolution images in real-time, on embedded systems and without notable accuracy loss, is still an open problem. 
However, human vision system provides intuition on how such solution can be achievable. On this basis, human vision has been found as foveal and active [26], [27]. Environment observation at high-resolution is achieved by the fovea, and only corresponds to 5 degrees of the total visual field [28]. Our complementary peripheral vision, which processes observations at a lower resolution, has a field of view of 110 degrees [29]. To track objects, our eyes perform quick saccadic movements, followed by smooth pursuit movements [30], [31]. In this context, when the focus is on an object, its peripheral surroundings can be simply ignored, a phenomenon known as inattentional blindness [32]. Hence, detecting and tracking objects is an active process, and the amount of information processed depends on the complexity and the dynamism of the scene, as well as on the attention of the observer. Besides, a fundamental advantage of human vision is that it relies on a stream of images, rather than on single images. Humans can rely on contextual cues and memory to supplement their understanding of the image. On this subject, some of the stated properties of human vision have led to novel neuromorphic sensor designs, such as Dynamic Vision Sensors (DVS), which react to events and decrease the amount of information processed. Although DVS-related research studies are still in early stages, they have provided promising results in the context of asynchronous object detection and tracking at high-speeds and low-power consumption [33], [34]. Conversely, this work, where a standard RGB camera sensor has been utilized, follows the idea of decreasing the amount of data to be processed, and examines the question of whether neural networks are similarly able to dynamically neglect image information when assisted by a memory and previous image context, providing a lower computation overhead.

An important property of video is that adjacent video frames are highly correlated, which opens up the possibility of decreasing the computation latency. During the process of object detection in a sequence, surrounding context of previous frame detection is prone to hold redundant content, which can be exploited in the current detection stage. Therefore, a simple idea is to keep a memory of previous extracted features and recompute only the ones corresponding to the region where the object was found in previous frame. To follow this idea, features from previous and current frame have to be fused in a synthetic feature map, which is used to compute the final detection. In this trend, based on the intuition that human peripheral vision may not add value while pursuing an object, our approach dynamically reuses feature context from previously detected frames to increase efficiency.

From these observations, we propose a simple but effective pipeline for efficient video object detection, illustrated in Fig. 1. Concretely, we introduce a novel inattentional framework, where peripheral detection context is dynamically reused to speed up inference latency while maintaining accuracy. The context features corresponding to keyframes are fused into a synthetic feature map, which is further refined

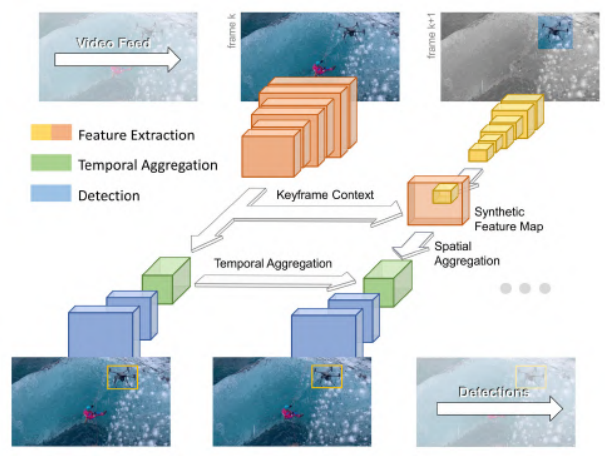

FIGURE 1. A schematic diagram of the proposed inattentional framework. The keyframe context is dynamically reused to reduce computation overhead, and temporal information is propagated by the temporal aggregator. Grey region in an image frame denotes pixels which are not being processed for the given frame.

based on its temporal structure. The temporal aggregation is carried out by a Convolutional Long-Short Term Memory (ConvLSTM) layer, and detections are generated by fusing context from previous frames with the new information of the current frame. Additionally, we show that the aggregation of our synthetic feature maps by the ConvLSTM contains within itself the information necessary to decide when the peripheral context has to be ignored. We learn an inattentional policy of when to use the full or synthetic feature map by formulating the task as a reinforcement learning problem.

In addition, the amount of attention a human observer gives to an object in a video sequence can be dynamic, modulating the inattentional blindness based on the one individual intentions. On the side of deep learning, neural networks are static structures which response commonly remains invariant during inference. This is due to the fact that during common network training stages, the parameters of a given loss function are not normally altered, narrowing the behavior of the network to a unique state of the potential distribution of loss functions. In this regard, recent advances reveal that a neural network can be trained not only over a unique loss function but over a distribution of them, as in You Only Train Once (YOTO) [35], [36]. Following this direction, the present work explores the possibility of extending this idea to the reinforcement learning paradigm, where the standard policy training involves defining a unique performance metric [37]-[39]. Under the context of the proposed application, the behavior of the inattentional policy network has been extended to dynamically provide different attention ratios, based on an inattentional parameter, which modulates the target performance metric on inference time.

While prior works, mostly flow [40]-[47] and recurrent [43], [48]-[52] methods, also provide approaches for fast video object detection with fast and slow detection stages, these approaches are based on processing the full image 
for every frame, incurring in a redundancy of computation and not exploiting the performance increase when the object appears reduced in the image. Conversely, our method takes advantage of the redundancy of context in video frames, increasing the performance when objects appear small in the image. Furthermore, approaches based on the reinforcement learning framework [49], provide policies trained over a unique performance metric i.e. reward function. Our policy network has been trained over a distribution of reward functions, providing not only a unique behavior but a distribution of them on inference time. Our method has been validated on the Imagenet VID 2015 [53] dataset and on our custom dataset, which has been made publicly available.

In summary, the contributions of the present work are as follows:

- We present a human-inspired inattentional framework for video object detection, where context features are dynamically reused in a synthetic feature map in order to reduce redundant computation, and their outputs are fused using a recurrent memory module.

- We introduce an adaptive inattentional policy where the decision over the context features computation is learned with deep reinforcement learning, which leads to a higher speed-accuracy trade-off.

- We demonstrate a successful application of YOTO [35] to the reinforcement learning paradigm, where a policy has been trained over a distribution of reward functions. To the authors knowledge, this is the first time this technique is applied to the reinforcement learning framework.

- The custom dataset used for validation of the framework has been made publicly available.

The remainder of the paper is organized as follows: Section III describes our approach and provides a detailed explanation about the inattentional fundamentals and the reward-conditional training, explaining the problem formulation and the system architecture. Section IV-B outlines the carried-out experiments and their corresponding results and Section $\mathrm{V}$ remarks on and discusses the most relevant experimentation outcomes. Finally, Section VI concludes the paper and indicates future lines of research.

\section{RELATED WORK}

This work proposes a video object detection framework and, to this aim, is aided by techniques of diverse nature, such as temporal aggregation or keyframe selection methods. As a consequence, there are several related works that are adjacent to the case under study.

\section{A. VIDEO POST-PROCESSING METHODS}

Early research for extending single-image object detection to the video domain was commonly focused on the generation of frame detection tracks, where current detection is linked to the previous ones in the track. Through these tracks, previous information is aggregated to improve the current frame object detection accuracy.

The Seq-nms algorithm proposed in [54] applies dynamic programming to find tracks and improve the confidence of weaker detections. Tubelets with Convolutional Neural Netowrks (TCNN) [55], [56] proposes a pipeline for detection propagation across frames via optical flow, and a tracking algorithm to refine scoring by finding tubelets. These initial strategies yielded accountable improvements in performance, but did not fundamentally change the basic per-frame detection techniques. In our work, we take advantage of the key idea of aiding current detection with previous detection context to decrease the computation time, while maintaining the accuracy.

\section{B. FEATURE AGGREGATION OVER TIME}

One key aspect which differentiates video from single-image detection, is the existence of encoded information that remains low-variant across several video frames. Consequently, there exist inter-frame features which can be aggregated and exploited to improve performance. In this regard, multiple techniques for stated feature aggregation have been explored in the literature.

In [40], intermmediate feature maps in a CNN were able to be temporally propagated across frames by means of optical flow. The Deep Feature Flow (DFF) framework [40] allows for feature propagation across multiple frames in order to compute detections on sparse keyframes, which alleviates the computational cost. Flow-Guided Feature Aggregation (FGFA) [41] further explored this idea by warping and averaging features from adjacent frames, which lead to acurracy improvements. Impression networks [42] provided a computation reduction by combining sparse "impression features", which stores low-variant and long-term information, with optical flow and warping aggregation. In [43] an efficient feature flow aggregation for different keyframe selection schemes is introduced. The Flow-Track framework [44] combines historical feature flows, warping, consine similarity and temporal attention for efficient feature aggregation achieving an increased performance. Some techniques, primarily meant for semantic object segmentation [45]-[47], also utilized warped feature flow maps to aid the final stages of the segmentation pipeline.

Recurrent architectures, such as Gated Recurrent Units (GRUs) or LSTM cells, have been integrated to enable the process of feature aggregation. In [43], a flow-guided GRU is proposed to effectively aggregate features on keyframes. Spatial-Temporal Memory Modules (STMMs) [48] were proposed to make better use of single-image detector weights pre-trained on large scale image datasets. ConvLSTMs variants have been more widely utilized for feature aggregation across frames [49]-[52]. Although ConvLSTM can fundamentally serve as a memory which is able to store mid-term information across frames [50], [51], it can be further extended to aggregate features from different extractors [49] or from an encoding of multiple frames [52]. 
Our ConvLSTM memory module is similar to [51], but serves an additional purpose of fusing features from real and synthetic feature maps, posing an additional challenge.

Furthermore, external memories can benefit the long-term information storage, which can be useful for feature aggregation in the video domain [57], [58]. Besides, some techniques integrate detection trackers to exploit temporal information between keyframe processing [59]-[61]. Other strategies rely on Locally-Weighted Deformable Neighbors (LWDNs) [62], Spatio-Temporal Sampling Networks (STSNs) [63], Motion History Image (MHI) [64], spatially variant convolutions [65] or cross-correlations [66] for feature propagation.

\section{ADAPTIVE KEYFRAME SELECTION}

Multiple approaches interleave processing pipelines with different computational budgets during the execution of the video object detection, in order to provide both fast and accurate solutions. This procedure commonly involves keyframe selection, where stated keyframe is processed by a more expensive but accurate pipeline. Despite keyframe selection can be carried out naively (i.e. fix rate), providing proper solutions [40], [43], [66], a more adaptive method for the case under study is potentially able to improve performance. For instance, to adapt to video input sources of variable complexity.

In [61], the optimal combination of detection and tracking stages is found in an offline trend for a specific video. In [43], a feature consistency indicator was used in a heuristic rule to select keyframes. A keyframe selection policy has been also incorporated to standard supervised learning schemes, such as the usage of low-level features for predicting rapid changes in the input [65], increase of complexity [45] or features quality [42].

Other techniques take advantage of the reinforcement learning framework for adaptive keyframe selection [47], [49], [60]. A policy-gradient reinforcement-learning approach [47] makes budget-aware processing by approximating the gradient of a non-decomposable and nondifferentiable objective. In [67], a learned policy is able to select when to update or to initiate a tracker. In [60], the reinforcement learning policy is guided to achieve a proper balance between detection and tracking. In [49] a light policy is used for balancing the execution pipeline between an expensive and a cheap feature extractor, achieving better results as compared to a random baseline. Our keyframe selection strategy has been inspired by the adaptive keyframe selection method found in [49]. In this direction, an adaptive selection policy has been learned within the reinforcement learning framework in order to select between real and synthetic feature maps (expensive and cheap pipeline, respectively) for detection. Furthermore, our complete policy has been trained over a distribution of reward functions, instead of a unique and constrained reward function, achieving proper performance throughout the whole domain.

\section{SINGLE-IMAGE ROI SELECTION METHODS}

A feed of video images opens the possibility of a potential increment of efficiency due to information redundancy across frames. Nevertheless, within the single-image context, there also exist regions which do not contain useful information to be exploited during the object detection task. In this regard, a notable amount of techniques have been explored in the literature. Stated strategies primarily aim at reducing the amount of information processed in an single image by selecting Regions of Interest (RoIs).

The generation of saliency maps can help during the RoI selection procedure. In the context of classical computer vision, log-spectrum [68], as well as a combination of image filters [69] (color, intensity, and orientation filters, among others) have been utilized for saliency computation. Additionally, RoI selection can be automated with standard supervised learning techniques. AutoFocus [70] forwards the full image through a cheap network to generate "chips" which are further processed in higher resolutions. In [71], a cropped image and its context is used in order to improve small object detection. AZ-Net [72] method zooms recursively into RoIs, which are likely to contain objects. In [73], RoIs are generated throughout an iterative process by the network, and a voting process is carried out to select the final regression. In [74], semantic and context information is used to find RoIs.

On the other hand, reinforcement learning has been notably applied to the problem of RoI selection. Dynamic zoom-in network [9] inputs a full low-resolution image and uses a reinforcement learning policy to select RoI to be further fine processed. In [75], an LSTM-based model is trained with reinforcement learning to give attention to certain RoI of the image in order to find small objects. In [76], [77], a reinforcement learning based sliding window approach finds an object in a few steps. Reinforcement learning based Region Proposal Network (RPN) [10] has also been explored to increase its efficiency of computation. In [78], [79], an agent selects the regions of the image which need to be processed at higher resolution or by a finer detector to reduce computation. In [80] RoIs are effectively selected based on the interdependence of objects with tree-structured reinforcement learning. In this work, RoIs are strongly related to the previous keyframe detection at a certain instant of time. Also, RoI size decision is based on the state of our ConvLSTM, being potentially assisted by the context of previous detected object in the sequence.

\section{THE INATTENTIONAL FRAMEWORK}

In this section, the proposed framework is described. First, the inattentional fundamentals are explained. Then, the adaptive inattentional policy as well as the reward-conditional training procedure are described in detail. The nomenclature and abbreviations used in the description of the inattentional framework are summarized in Table 1.

\section{A. INATTENTIONAL FUNDAMENTALS}

Following the intuition provided by the inattentional blindness phenomenon [32], our solution is approached as a 


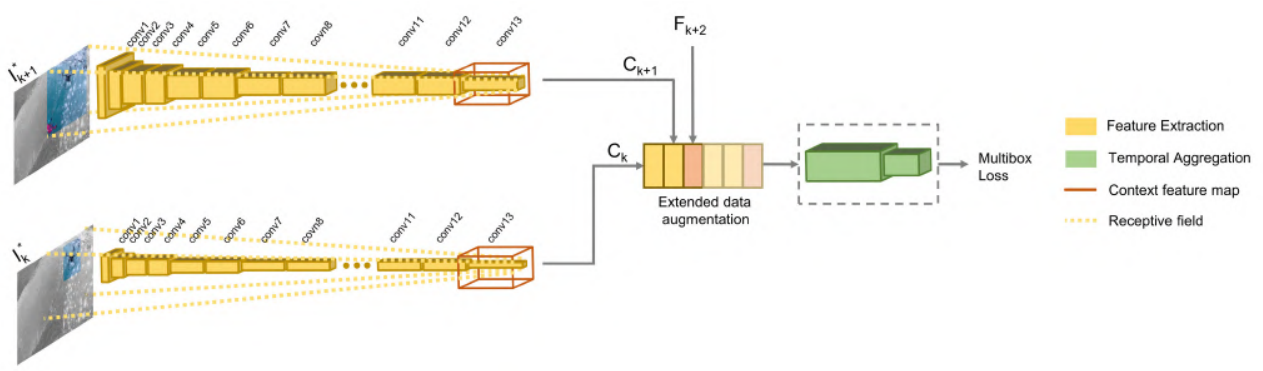

FIGURE 2. Structure of the extended data augmentation technique. The architecture shown corresponds to MobileNetV1 feature extractor. The receptive field in a deep layer of a standard CNN is usually a notable region of the input image. In order to further reduce the computation overhead, image crops $I_{k}^{*}$ have not been selected to match the required receptive field. To tackle this issue, the resulting feature maps $C_{k}$ have been included in the augmented training data for generality of the temporal aggregator.

TABLE 1. Table of abbreviations and nomenclature in the context of the proposed inattentional framework.

\begin{tabular}{ll} 
Symbol & Definition \\
\hline $\mathbf{f}$ & Feature extractor \\
$\mathbf{c}$ & Context aggregator \\
$\mathbf{a}$ & Temporal aggregator \\
$\mathbf{d}$ & Detector \\
$\mathcal{V}$ & Video sequence \\
$I_{k}$ & $k$-th image frame \\
$I_{k}^{*}$ & $k$-th inattentional image frame (image \\
$F_{k}^{c}$ & crop) \\
$F_{k}^{*}$ & Feature map context (output from f) \\
$C_{k}$ & put from $\mathbf{f}$ ) \\
$A_{k}$ & Tynthetic feature map (output from c) \\
& (output from a) \\
$D_{k}$ & Detections (output from d) \\
$\lambda_{0}$ & Reward-conditional parameter
\end{tabular}

dynamic context reuse across an image sequence $\mathcal{V}=$ $\left\{I_{0}, I_{1}, I_{2}, \ldots, I_{n}\right\}$. Indeed, the phenomenon of momentarily ignoring context, while detecting an object, can be effectively reformulated as a context reuse, since reusing previous context is equivalent to avoiding current context computation. Besides, our framework is restricted to the online setting where only $\left\{I_{0}, I_{1}, \ldots, I_{k}\right\}$ are available during the computation of the $k$-th detection.

In order to materialize this concept into the deep learning paradigm, four main components have been defined in the present framework: a feature extractor $\mathbf{f}$, a context aggregator c, a temporal aggregator a and a detector $\mathbf{d}$. The context is defined as a subset of the feature maps generated by the feature extractor $\mathbf{f}$ on a keyframe $k$. In this direction, our approach interleaves full keyframe computation and partial frame (inattentional frame) computation with context aggregation (see Fig. 2 and Fig. 3) to provide an intermmediate representation. The frame-level features are then temporally aggregated and refined using a recurrent architecture. Finally, an SSD-style [17] detection pipeline is applied on the refined feature map to obtain bounding box results.

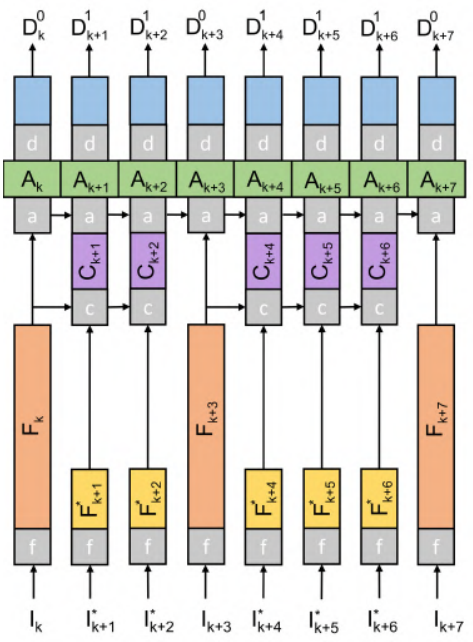

FIGURE 3. A block diagram of our adaptive keyframe selection technique with all the components of the inattentional framework included, where $f$ stands for feature extractor, $\boldsymbol{c}$ is the context aggregator, $a$ depicts the temporal aggregator and $\boldsymbol{d}$ represents the detector. For clarity, coloured blocks correspond to tensors and grey blocks correspond to components of the system. The length of the tensor denotes time consumption but it is not to scale. The context of a keyframe $F_{k}^{c}$ is not being modified throughout the pipeline of context aggregators.

Each step of the processing pipeline can be defined as a function mapping. The feature extractor $\mathbf{f}: \mathbb{R}^{I} \rightarrow \mathbb{R}^{F}$, maps the image space into an encoded feature space $\mathbb{R}^{F}$. The context aggregator $\mathbf{c}: \mathbb{R}^{F} \times \mathbb{R}^{F} \rightarrow \mathbb{R}^{C}$ optionally composes a synthetic feature map $C_{k}$, based on previous keyframe context $F_{k}^{c}$ and current reduced feature map $F_{k}^{*}$. The temporal aggregator a: $\mathbb{R}^{F} \times \mathbb{R}^{S} \rightarrow \mathbb{R}^{A} \times \mathbb{R}^{S}$ or $\mathbb{R}^{C} \times \mathbb{R}^{S} \rightarrow \mathbb{R}^{A} \times \mathbb{R}^{S}$, refines the generated feature map (either full $\mathbb{R}^{F}$ or synthetic $\mathbb{R}^{C}$ ) based on previous temporal information cues, compute its state $\mathbb{R}^{S}$ 
and outputs an updated feature space $\mathbb{R}^{A}$. The SSD detector d: $\mathbb{R}^{A} \rightarrow \mathbb{R}^{D}$, maps the aggregated feature space into final detection predictions. In order to obtain detections $D_{k}$, one may compute $A_{k}$ by $\mathbf{a}\left(\mathbf{f}\left(I_{k}\right), s_{k-1}\right)$ or $\mathbf{a}\left(\mathbf{c}\left(\mathbf{f}\left(I_{k}^{*}\right), F_{k}^{c}\right), s_{k-1}\right)$, where $s_{k-1}$ is the previous frame state and, $I_{k}$ and $I_{k}^{*}$ are the full image or cropped image for the $k$-th frame, respectively. The final detections $D_{k}$ are obtained as $D_{k}=\mathbf{d}\left(A_{k}\right)$.

The context aggregator $\mathbf{c}$ is a key component in the inattentional framework, as it is in charge of reusing context from previous keyframes, in order to provide a more efficient performance while reducing computation overhead. Specifically, reusing context requires fusing the current feature map $F_{k}^{*}$ with the context feature map $F_{k}^{c}$. Since the context is related to the object in the image space, one simple approach for fusing feature maps is to calculate the precise position of a feature map corresponding to an image crop $I_{k}^{*}$ inside a context feature map $F_{k}^{c}$ corresponding to a full keyframe $I_{k-n}$. Furthermore, in order to precisely select the context crop in the image space (image crop $I_{k}^{*}$ ), previous detection $D_{k-1}$ and the particular receptive field of $F_{k}$ have to be taken into consideration. However, the receptive field of a given layer unit is crucial to generate a feature map $F_{k}^{*}$ which can be directly placed (without distortion) in the context feature map $F_{k}^{c}$. In this respect, it is important to highlight that the receptive field size of a deep layer in a neural network normally covers a notable percentage of the input image (the receptive field size of the final layer increases with the depth and the striding of the network). At this point, a trade-off, between the image crop $I_{k}^{*}$ size and the computation overhead stands out, since in order to account the full size of the receptive field, the image crop can be increased for a certain object, incurring in more computation overhead, and vice versa.

As the present framework targets computation efficiency, the amount of aggregated context has to be maximized in order to reduce the computation overhead. This fact leads to the generation of image crops $I_{k}^{*}$ with a small size which may not be matching the required receptive field for the feature map $F_{k}$ (see Fig. 2). In this scenario, the aggregated feature map $C_{k}$ can exhibit artifacts around the area where $F_{k}^{*}$ was placed, resulting in a lower accuracy of the complete approach. To tackle this issue, the temporal aggregator a has been also trained to adapt $\mathbb{R}^{C} \rightarrow \mathbb{R}^{F}$ domains via extended feature map augmentation during training. Thus, the temporal aggregator has been trained with augmented data which aids the detection with synthetic feature maps (refer to Training Methodology in Section IV-A). In this context, the temporal aggregator not only aggregates temporal video information, but also refines feature maps from different nature (full and synthetic).

\section{B. ADAPTIVE INATTENTIONAL POLICY}

Although context aggregation at random intervals is able to provide competitive results in terms of latency reduction while maintaining accuracy (refer to Section IV-B), a natural question is whether an adaptive policy can improve these results by using the state of the system as well as the complexity of the input as information sources. In this regard, a variable amount of inattention can be provided to the context, based on the uncertainty of past detections and the object dynamism in a given sequence. Accordingly, a novel adaptive inattentional policy has been proposed, using formulation of the reinforcement learning paradigm.

In reinforcement learning, an agent interacts with an environment, seeking to find the maximum accumulated reward over time. To formulate the reinforcement learning problem, it is necessary to define an action space $\mathbb{A}$, a state space $\mathbb{S}$, and a reward function $r$. In the proposed inattentional framework, the inattentional policy has the ability of executing two actions: it can decide whether to execute the full expensive pipeline or, on the contrary, to select the faster context aggregation pipeline (with the potential loss on accuracy). In this regard, the discrete action space $\mathbb{A}$ is defined as $\mathbb{A}=a$ with $a \in\{0,1\}$.

Additionally, our proposed policy is meant to examine the temporal aggregator state in order to find insights about the uncertainty of the detection, as well as about abrupt context changes in order to perform the best possible action, leading to an optimum policy $\pi^{*}\left(s_{t} \mid \theta\right)$. Following this idea, the state space $\mathbb{S}$ is defined as

$$
\mathbb{S}=\left(c_{k}, h_{k}, c_{k}-c_{k-1}, h_{k}-h_{k-1}, \rho_{k}, b_{k-1}, \psi\right)
$$

where $c_{k}$ and $c_{k-1}$ are the current and previous ConvLSTM cell states, respectively; $h_{k}$ and $h_{k-1}$ are the current and previous ConvLSTM hidden states, respectively; $\rho_{k}$ is an action history vector and has been empirically found to work properly with size $20, b_{k-1}$ is the normalized previous $I_{k}^{*} \operatorname{RoI}$ (it is the normalized full image RoI if previous frame was a keyframe), and $\psi$ is the inattentional factor. The inclusion of $\left(c_{k}-c_{k-1}\right)$ and $\left(h_{k}-h_{k-1}\right)$ helps detecting changes in the temporal aggregator state. Also, $\rho_{k}$ and $b_{k-1}$ make the agent aware of its previous actions and context size, in order to avoid excessively running the context aggregation pipeline. The size of the state with the current architecture is 102,425 continuous variables.

The reward function $r$ is a crucial component in the reinforcement learning framework. Indeed, it can either speed up training or, conversely, a naive design can completely prevent the agent from learning. Our reward must reflect the intention of finding balance between running the context aggregation pipeline as much as possible while maintaining accuracy. In this work, the reward function has been adapted from [49] as

$$
r= \begin{cases}\min _{i}\left(L\left(D^{i}\right)\right)-L\left(D^{0}\right) & a=0 \\ \psi+\min _{i}\left(L\left(D^{i}\right)\right)-L\left(D^{1}\right) & a=1\end{cases}
$$

where $D^{0}$ and $D^{1}$ are the detection results through the expensive pipeline (full image) and cheap pipeline (context aggregation), respectively; $L(\cdot)$ is the Multibox loss [81] computation and $\psi$ corresponds to the inattentional factor. The inattentional factor $\psi$ is an important component of the reward function. It is a scalar value which can potentially 
encourage the agent taking the cheap pipeline, even when its cost remains higher. The definition of the $\psi$ value is decisive for the final behavior of the agent.

\section{REWARD-CONDITIONAL TRAINING}

Conventional methods for training machine learning algorithms define one or more differentiable cost functions to perform the gradient propagation to the input(s). Typically, cost functions include static parameters which are able to modulate training and/or inference performance, leading to a static behavior of the network at inference time. Stated cost function parameters can be tuned based on experience, trialand-error, grid search or more advanced methods. However, recent techniques have revealed a convolutional network can be trained over the whole distribution of parameterized cost functions, as shown in (3), enabling the possibility of conditioning the network on a subset of parameters at inference time (YOTO [35]).

$$
\theta^{*}=\underset{\theta}{\arg \min } \sum_{i=1}^{n} L\left(\mathbf{y}_{i}, F\left(\mathbf{x}_{\mathbf{i}}, \theta, \lambda_{\mathbf{i}}\right), \lambda_{i}\right), \quad \lambda_{i} \sim P_{\lambda}
$$

where $L$ represent one of the $n$ cost functions, $\lambda_{i}$ are the cost function(s) parameters and $\theta$ the model parameters. Also, in [35], Feature-wise Linear Modulation (FiLM) [36] is used to condition the network on the loss parameters. This technique has been applied in [35] to fully convolutional models in a supervised and semi-supervised trend, e.g. $\beta$-variational autoencoder, image compression and style transfer.

In this work, we extend the loss-conditional training framework [35] by proposing a novel reward-conditional training, under the reinforcement learning formulation. As in the supervised or semi-supervised case, a common agent in reinforcement learning is trained over a parameterized performance function, in this case a reward function, which has to be properly adjusted to encourage the desired behavior. Once the reward function and its parameters are defined, the agent behavior converges to a subset of possible behaviors within the distribution of the reward function parameters. Instead of defining a unique parameterized reward function, we propose to condition the network policy $\pi^{*}\left(s_{t} \mid \theta, \lambda_{i}\right)$ on the whole distribution of parameterized reward functions, as in (4) and (5).

$$
\begin{aligned}
\pi^{*}\left(s_{t} \mid \theta, \lambda_{i}\right) & =\underset{a_{t} \in \mathbb{A}}{\arg \max } Q\left(s_{t}, a_{t} \mid \theta, \lambda_{i}\right), \quad \lambda_{i} \sim P_{\lambda} \\
Q\left(s_{t}, a_{t} \mid \theta, \lambda_{i}\right) & =\mathbb{E}_{\pi}\left[R_{t}\left(\lambda_{i}\right)\right] \\
& =\mathbb{E}_{\pi}\left[\sum_{k=0}^{\infty} \gamma_{r}^{k} r\left(\lambda_{i}\right)_{t+k+1}\right]
\end{aligned}
$$

where $\theta$ corresponds to the model function parameters, $\lambda_{i}$ are the reward-conditional parameters, $\gamma_{r}$ is the discount factor and $r\left(\lambda_{i}\right)$ denotes the distribution of conditioned reward functions.

For the case under study, our reward function is composed of one important parameter $\psi=\lambda_{0}$, which balances the amount of accuracy an agent is able to sacrifice when running

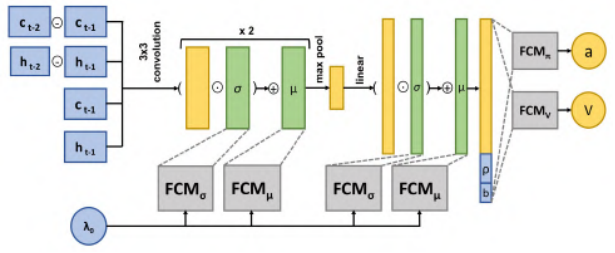

FIGURE 4. The architecture of the inattentional policy network. The state tensors have been depicted in dark blue, as well as the $\lambda_{0}$ reward-conditional parameter. Part of the state $(\rho$ and $b)$ is being appended in the last fully connected layer of the network. The reward-conditional parameter has been integrated by applying FiLM technique to the tensors represented in dark green.

both expensive and cheap pipelines. $\lambda_{0}$ has been selected as the reward-conditional parameter. Analogously to the reward expression shown in (2), the formulation of the final conditioned reward function is illustrated in (6) for clarity.

$$
r\left(\lambda_{0}\right)= \begin{cases}\min _{i}\left(L\left(D^{i}\right)\right)-L\left(D^{0}\right) & a=0 \\ \lambda_{0}+\min _{i}\left(L\left(D^{i}\right)\right)-L\left(D^{1}\right) & a=1\end{cases}
$$

where $\lambda_{0} \sim P_{\lambda}$ and $P_{\lambda}$ denotes a log uniform distribution of probability with $\lambda_{0} \in[0,2]$.

In our experiments, the policy $\pi\left(s_{t} \mid \theta, \lambda_{0}\right)$ is built on a light convolutional backbone and two Fully Connected Models (FCMs), which output both the action and the value (see Fig. 4). To condition the network on the reward parameters, we use FiLM. We have selected the convolutional layers and one fully-connected layer to be conditioned by $\lambda_{0}$. Assume a given feature map $\mathbf{f}$ of dimensions $W \times H \times C$, with $W$ and $H$ corresponding to the spatial dimensions and $C$ to the channels. The scalar value $\lambda_{0}$ is fed to two FCMs, $M_{\sigma}$ and $M_{\mu}$, to generate two vectors, $\sigma$ and $\mu$ of dimensionality $C$ each. We then multiply the feature map channel-wise by $\sigma$ and add $\mu$ to get the transformed feature map $\hat{\mathbf{f}}$, as in (7).

$$
\hat{f}_{i j k}=\sigma_{k} \circ f_{i j k}+\mu_{k}
$$

where $\sigma=M_{\sigma}\left(\lambda_{0}\right)$ and $\mu=M_{\mu}\left(\lambda_{0}\right)$, and $i j k$ correspond to the feature location within the $W \times H \times C$ scope. Following this technique, the policy can be fully learned and conditioned with $\lambda_{0}$ at inference time, providing different attention ratios while maintaining accuracy. The reward-conditional training framework has been validated for the challenging application under study. However, a complete analysis and validation of the reward-conditional framework is out of the scope of this work and have been proposed for future work.

\section{SYSTEM ARCHITECTURE}

The architecture of the system is designed with the aim of maximizing the computation efficiency. MobileNetV1 [16] and MobileNetV2 [17] architectures have been utilized as feature extractors $\mathbf{f}$ or backbones. MobileNetV1 and MobileNetV2 backbones have been slightly modified, 
to standardize their output size, with respect to the feature aggregator input size. The conv14 layer in MobileNetV1 has been removed. In MobileNetV2, the stride has been set to 1 in the 6-th bottleneck layer, and the number of channels has been reduced to 512 in the last conv layer (prior to the classification stage in the original model). For both backbones, a depth-multiplier $\alpha=1.0$ has been selected for experimentation, which allows for a baseline model capacity to extract representative features in the context of the presented datasets. With proper model capacity, the models are less biased, and the performance of our additional adaptive policy, whose primary inputs are the stated extracted features, can be adequately validated. Nevertheless, our framework provides sufficient generality to use arbitrary feature extractor designs ( $\alpha>0$, ResNet variants [82], etc.). The receptive field size of MobileNetV1 and MobileNetV2 for the last layer in each feature extractor is $219 \mathrm{px} \times 219 \mathrm{px}$ and $395 \mathrm{px} \times 395 \mathrm{px}$, respectively.

The feature aggregator $\mathbf{a}$ is composed of a ConvLSTM cell, which is in charge of aggregating both time and spatial features. The ConvLSTM cell has been implemented as in [50]. The bottleneck design of the ConvLSTM and its depth-wise separable convolutions reduce the computational costs. This fact is crucial, since the temporal aggregator has to be executed for every frame of the sequence. The size of the hidden and cell states of the ConvLSTM have been adapted based on the input image size. For the detector d, an SSDLite architecture [17] has been adopted, with separable convolutions and depth-wise channel size in all layers. Finally, the policy network $\pi\left(s_{t} \mid \theta, \lambda_{0}\right)$ has been trained with Proximal Policy Optimization (PPO) [37] algorithm, which is an actor-critic algorithm. The architecture has been shown in Table 2, without accounting the extra FCMs required for the reward-conditional training for clarity. The FCMs corresponding to the reward-conditional training are shallow fully-connected models with one hidden layer each (varying from 128 to 512 units) and one output layer (varying from 64 to 512 units). The layers corresponding to the value network, which shares weights with the policy network, have been also represented in Table 2 .

\section{EXPERIMENTS AND RESULTS}

In the following section, the proposed inattentional framework has been thoroughly tested and validated. The training methodology section describes the staged training procedure for the presented system. The results section shows the experimentation outcome corresponding to every component of our system in a wide variety of scenarios. Finally, the discussion section interrelates the results to extract insights on our framework design.

\section{A. TRAINING METHODOLOGY}

As explained in previous sections, the proposed system is composed of several components. In this context, the training is divided into three phases: feature extractor (and detector), temporal aggregator (and detector) and adaptive inattentional
TABLE 2. The proposed policy network architecture. For clarity, the layers corresponding to the reward-conditional training FCMs have not been included. The layers from conv1 to $\mathrm{fcl}$ are shared layers between the policy and the value models. The fully connected layers are relative to a $320 \times 320$ input image resolution.

\begin{tabular}{c|c|c} 
Layer & Size & Stride \\
\hline conv1 & $3 \times 3 \times 1024 \times 128$ & 1 \\
\hline conv2 & $3 \times 3 \times 128 \times 128$ & 1 \\
\hline pool1 & $2 \times 2 \times 128$ & 2 \\
\hline fc1 & $(3200+20+4) \times 512$ & - \\
\hline pi_fc1 & $512 \times 128$ & - \\
\hline pi_fc2 & $128 \times 128$ & - \\
\hline pi_fc3 & $128 \times 128$ & - \\
\hline pi_fc4 & $128 \times 1$ & - \\
\hline v_fc1 & $512 \times 128$ & - \\
\hline v_fc2 & $128 \times 128$ & - \\
\hline v_fc3 & $128 \times 128$ & - \\
\hline v_fc4 & $128 \times 1$ & - \\
\hline
\end{tabular}

policy training. Although the complete system can potentially be trained end-to-end, we approached a staged training pipeline in order to avoid convergence issues. The feature extractor, the temporal aggregator and detector have been trained in PyTorch [83], while the adaptive inattentional policy has been trained in Tensorflow [84]. The GPU used for training has been an Nvidia GeForce RTX 2080 Ti. All the models have been trained on a subset of 260,844 images from Imagenet VID 2015 dataset [53] and on 29,500 images from a custom Multirotor Aerial Vehicles VID ${ }^{1}$ (MAV-VID) dataset, which has been made publicly available (validation sets are composed of 3,139 and 10,732 images, respectively). The Imagenet VID 2015 dataset has been reduced by selecting the 7-th smallest classes in terms of bounding boxes area (varying from $18 \%$ to $30 \%$ of the full-size image). The resulting experiments provide the generality of the public Imagenet dataset, and additionally represent the ultimate goal of the proposed framework, which is reusing context features when images context is notable across frames. Furthermore, our custom MAV-VID dataset constitutes the target application domain for the case under study, where the object to be detected appears normally small-sized in the image plane, such as the case of flying multirotor vehicles.

\section{1) FEATURE EXTRACTOR}

In the first stage, the feature extractor has been trained in conjunction with the SSD detector header, in order to extract robust features to be used by the following stages. Random single images from the video sequences and a batch of 16 images have been used. Adam has been selected as the optimizer, with a learning rate of $10^{-4}$. We use an input resolution of $320 \times 320$ and reduce on plateau learning rate scheduler. We include hard negative mining and data augmentation as described in [81]. The original hard negative mining approach has been adjusted by allowing a ratio of 10 negative examples for each positive while scaling each negative loss by

\footnotetext{
${ }^{1}$ https://www.kaggle.com/alejodosr/multirotor-aerial-vehicle-vidmavvid-dataset
} 
0.3. The validation phase is carried out through the whole set of validation sequences of the datasets.

\section{2) TEMPORAL AGGREGATOR}

In the second stage, the feature extractor weights have been frozen during training. For MobileNetV1, the conv14 layer has been removed in order to inject the ConvLSTM layer. Again, the temporal aggregator is trained along with the SSD detection headers. We sample random sequences (of 10 frames each) from the training set for training. We unroll the LSTM to 10 frames in which we apply the same data augmentation techniques as in previous stage. The same data augmentation transformation is applied to every frame of each sequence in order to avoid missing the correlation between consecutive frames. We use a batch of 50 sequences and an image input size of $320 \times 320$.

Additionally, an extended data augmentation technique has been included to account for the structure of the synthetic feature maps. As explained in Section III-A, due to the procedure of context reuse, where a reduced feature map is placed in a context feature map, artifacts can appear close to the placement volume. In order to tackle this effect, during training, the feature map which results from the feature extraction stage is randomly modified with the ground-truth context of previous frame. We set a 0.01 probability for a feature map to be modified by this technique. Adam optimizer has been utilized with a learning rate of $10^{-4}$ and reduce on plateau learning rate scheduler. The validation is carried out through the validation sequences in each dataset.

\section{3) ADAPTIVE INATTENTIONAL POLICY}

The adaptive inattetional policy has been trained using PPO algorithm. PPO is a sample-efficient and actor-critic deep reinforcement learning algorithm. A clip range of 0.1 , trajectories of 128 steps and minibatches of 4 elements have been utilized. The policy network has been trained with Adam and a learning rate of $2.5 \cdot 10^{-4}$ (refer to the code repository Supplementary Material section for remaining hyperparameters definition). The observation state has been normalized in the environment based on its mean and variance through time (at a certain number of steps, normalization parameters calculation is stopped). The reward has not been normalized, since its original design is meant for relative performance signaling. $\lambda_{0}$ parameter has been sampled from a log-uniform distribution every 10 sequences. Training has been carried out with the validation set of each dataset. In order to provide enough variability and avoid overfitting, a random transform as in [81] has been applied to every sequence. During testing, the random transform is not applied to allow for comparison.

\section{B. RESULTS}

For all results, the standard Imagenet VID accuracy metric is reported, mean Average Precision mAP@0.5 IOU. The latency of the networks in milliseconds (ms) and the average Frames per Second (FPS) of the complete approach is provided. The number of parameters is reported as benchmarks for efficiency. Also, the power consumption in Watts (W) and the energy efficiency in FPS/W are included for every experiment, as reported in [11]-[13].

The validation tests have been performed in two different GPUs, an Nvidia GeForce RTX 2080 Ti desktop GPU and an Nvidia Volta embedded GPU inside an Nvidia Jetson AGX Xavier platform. Also, since the latency reduction greatly stands out when the parallelization capacity is decreased, such as the case of desktop and embedded CPUs, the proposed system has been further tested and validated in an Intel Core i7-9700K@3.60GHz desktop CPU and a ARMv8.2@1.377GHz embedded CPU. All the tests have been performed in Python 3.6 and Ubuntu 18.04 operating system. The code has been made publicly available (see Supplementary Material).

In Table 3 and Table 4, the results corresponding to MobileNetV1 feature extractor for both Imagenet VID 2015 and MAV-VID dataset are shown. In addition, in Table 5 and Table 6 , the results corresponding to MobileNetV2 feature extractor for both Imagenet VID 2015 and MAV-VID dataset are also depicted.

In order to further validate the performance of the proposed inattentional policy, a comparison with a random baseline for a wide variety of scenarios has been carried out. In Fig. 5 the trade-off between the number of inattentional frames executed and the resulting mAP is illustrated for both datasets and feature extractors. For every value of the reward-conditional parameter $\lambda_{0}$, there is an overall percentage of inattentional frames executed. In order to be able to compare it to a random baseline, a random policy has been executed the same exact amount of inattentional frames. Also, the original $\mathrm{mAP}$ (with no inattentional frames involved) is included. Every test has been performed 5 times each, for both policies, and the average results have been plotted.

Finally, four application cases have been illustrated in Fig. 6. Two complex scenarios, due to object high-motion speed within the image plane or environmental complexity (camera pointing to the sun); and two simple (or static) scenarios, where the object stays almost static within the image plane, have been additionally depicted.

\section{DISCUSSION}

The thorough testing and validation of the proposed inattentional system describes a proper framework to adaptively reduce computation overhead in the context of video object detection. A wide variety of scenarios have been tested for two feature extractors (MobileNetV1 and MobileNetV2) and two datasets (Imagenet VID 2015 and MAV-VID). Regarding Table 3, 4, 5 and 6, both architectures provide state-of-the-art mAP@0.5IOU in the Imagenet VID 2015 dataset, resulting in 0.5236 and 0.4924 for MobileNetV1 and MobileNetV2 feature extractors, respectively. Also, the mAP results in our custom MAV-VID dataset are notable, with 0.9398 and 0.9453 for MobileNetV1 and MobileNetV2 feature extractors, respectively. MobileNetV1 provided higher mAP in 
TABLE 3. Results using the MobileNetV1 feature extractor on the ImageNet VID 2015 dataset. The mean Average Precision (mAP), KeyFrame (KF) and Inattentional Frame (IF) latency, as well as the average runtime FPS are provided. Also, the number of parameters of the models has been included. The FPS ratio with respect to the base FPS value (without the inattentional policy) has been also included for clarity. Additionally, power consumption and energy efficiency have been provided for every experiment.

\begin{tabular}{|c|c|c|c|c|c|c|c|c|c|}
\hline Model & Platform & $\mathrm{mAP}$ & $\begin{array}{l}\mathrm{KF} \\
(\mathrm{ms})\end{array}$ & $\begin{array}{c}\text { IF } \\
(\mathrm{ms})\end{array}$ & FPS & $\begin{array}{l}\text { FPS } \\
\text { Ratio }\end{array}$ & $\begin{array}{c}\text { Params } \\
(\mathrm{M})\end{array}$ & $\begin{array}{c}\text { Power } \\
(\mathrm{W})\end{array}$ & $\begin{array}{c}\text { Efficiency } \\
\text { (FPS/W) }\end{array}$ \\
\hline MobileNetV1-SSDLite & $\begin{array}{l}\text { GPU (Desktop) } \\
\text { GPU (Xavier) } \\
\text { CPU (Desktop) } \\
\text { CPU (Xavier) }\end{array}$ & 0.4824 & $\begin{array}{c}20.67 \\
84.53 \\
159.73 \\
7018.31\end{array}$ & $\begin{array}{l}- \\
- \\
-\end{array}$ & $\begin{array}{l}48.37 \\
11.83 \\
26.26 \\
0.142\end{array}$ & $\begin{array}{l}- \\
- \\
- \\
-\end{array}$ & 4.48 & $\begin{array}{l}128.3 \\
4.19 \\
99.1 \\
2.91\end{array}$ & $\begin{array}{c}0.377 \\
2.82 \\
0.264 \\
0.048\end{array}$ \\
\hline MobileNetV1-ConvLSTM-SSDLite & $\begin{array}{l}\text { GPU (Desktop) } \\
\text { GPU (Xavier) } \\
\text { CPU (Desktop) } \\
\text { CPU (Xavier) }\end{array}$ & 0.5236 & $\begin{array}{c}19.33 \\
83.73 \\
39.53 \\
6378.85 \\
\end{array}$ & $\begin{array}{l}- \\
- \\
- \\
-\end{array}$ & $\begin{array}{l}51.73 \\
11.94 \\
25.29 \\
0.156 \\
\end{array}$ & $\begin{array}{l}- \\
- \\
- \\
-\end{array}$ & 3.3 & $\begin{array}{l}126.8 \\
3.82 \\
98.9 \\
2.87 \\
\end{array}$ & $\begin{array}{c}0.407 \\
3.12 \\
0.255 \\
0.054 \\
\end{array}$ \\
\hline MobileNetV1-ConvLSTM-SSDLite $\left[\lambda_{0}=0.01\right]$ & $\begin{array}{l}\text { GPU (Desktop) } \\
\text { GPU (Xavier) } \\
\text { CPU (Desktop) } \\
\text { CPU (Xavier) }\end{array}$ & 0.5235 & $\begin{array}{c}19.59 \\
83.33 \\
39.62 \\
6512.13\end{array}$ & $\begin{array}{c}18.78 \\
76.63 \\
25.01 \\
4132.13\end{array}$ & $\begin{array}{c}51.91 \\
12.4 \\
29.65 \\
0.1801\end{array}$ & $\begin{array}{l}1.0 \\
1.03 \\
1.17 \\
1.15\end{array}$ & 5.5 & $\begin{array}{c}141.5 \\
4.25 \\
103.1 \\
3.03\end{array}$ & $\begin{array}{c}0.366 \\
2.91 \\
0.287 \\
0.059\end{array}$ \\
\hline MobileNetV1-ConvLSTM-SSDLite $\left[\lambda_{0}=0.5\right]$ & $\begin{array}{l}\text { GPU (Desktop) } \\
\text { GPU (Xavier) } \\
\text { CPU (Desktop) } \\
\text { CPU (Xavier) }\end{array}$ & 0.4964 & $\begin{array}{c}19.78 \\
82.73 \\
39.12 \\
6532.76\end{array}$ & $\begin{array}{c}18.64 \\
77.36 \\
25.13 \\
4189.74\end{array}$ & $\begin{array}{c}52.67 \\
12.66 \\
34.06 \\
0.2041\end{array}$ & $\begin{array}{c}1.0 \\
1.06 \\
1.34 \\
1.3\end{array}$ & 5.5 & $\begin{array}{c}141.5 \\
4.25 \\
103.1 \\
3.03\end{array}$ & $\begin{array}{c}0.372 \\
2.97 \\
0.33 \\
0.067\end{array}$ \\
\hline MobileNetV1-ConvLSTM-SSDLite $\left[\lambda_{0}=1.2\right]$ & $\begin{array}{l}\text { GPU (Desktop) } \\
\text { GPU (Xavier) } \\
\text { CPU (Desktop) } \\
\text { CPU (Xavier) }\end{array}$ & 0.4781 & $\begin{array}{c}19.99 \\
83.1 \\
39.33 \\
6580.88\end{array}$ & $\begin{array}{c}18.68 \\
75.15 \\
25.07 \\
4063.28\end{array}$ & $\begin{array}{c}52.85 \\
13.01 \\
36.11 \\
0.2209\end{array}$ & $\begin{array}{l}1.0 \\
1.08 \\
1.42 \\
1.41\end{array}$ & 5.5 & $\begin{array}{c}141.5 \\
4.25 \\
103.1 \\
3.03\end{array}$ & $\begin{array}{c}0.373 \\
3.06 \\
0.35 \\
0.072 \\
\end{array}$ \\
\hline MobileNetV1-ConvLSTM-SSDLite $\left[\lambda_{0}=2.0\right]$ & $\begin{array}{l}\text { GPU (Desktop) } \\
\text { GPU (Xavier) } \\
\text { CPU (Desktop) } \\
\text { CPU (Xavier) }\end{array}$ & 0.4373 & $\begin{array}{c}19.91 \\
83.46 \\
39.89 \\
6593.12\end{array}$ & $\begin{array}{c}18.23 \\
76.36 \\
25.45 \\
4028.2\end{array}$ & $\begin{array}{c}\mathbf{5 4 . 4 8} \\
13.15 \\
37.73 \\
0.2372\end{array}$ & \begin{tabular}{c|}
1.05 \\
1.1 \\
1.49 \\
1.52
\end{tabular} & 5.5 & $\begin{array}{c}141.5 \\
4.25 \\
103.1 \\
3.03\end{array}$ & \begin{tabular}{c|c|}
0.385 \\
3.09 \\
0.365 \\
0.078
\end{tabular} \\
\hline
\end{tabular}

TABLE 4. Results using the MobileNetV1 feature extractor on the MAV-VID dataset. The mean Average Precision (mAP), KeyFrame (KF) and Inattentional Frame (IF) latency, as well as the average runtime FPS are provided. The FPS ratio with respect to the base FPS value (without the inattentional policy) has been also included for clarity. Additionally, power consumption and energy efficiency have been provided for every experiment.

\begin{tabular}{|c|c|c|c|c|c|c|c|c|}
\hline Model & Platform & mAP & $\begin{array}{c}\mathrm{KF} \\
(\mathrm{ms})\end{array}$ & $\begin{array}{c}\text { IF } \\
(\mathrm{ms})\end{array}$ & FPS & $\begin{array}{l}\text { FPS } \\
\text { Ratio }\end{array}$ & $\begin{array}{c}\text { Power } \\
\text { (W) }\end{array}$ & $\begin{array}{c}\text { Efficiency } \\
\text { (FPS/W) }\end{array}$ \\
\hline MobileNetV1-SSDLite & $\begin{array}{l}\text { GPU (Desktop) } \\
\text { GPU (Xavier) } \\
\text { CPU (Desktop) } \\
\text { CPU (Xavier) }\end{array}$ & 0.9183 & $\begin{array}{c}18.85 \\
90.66 \\
122.58 \\
6406.07\end{array}$ & $\begin{array}{l}- \\
- \\
- \\
-\end{array}$ & $\begin{array}{c}53.05 \\
11.03 \\
8.15 \\
0.156\end{array}$ & $\begin{array}{l}- \\
- \\
-\end{array}$ & $\begin{array}{l}128.2 \\
4.16 \\
99.0 \\
2.93\end{array}$ & $\begin{array}{c}0.413 \\
2.65 \\
0.082 \\
0.053\end{array}$ \\
\hline MobileNetV1-ConvLSTM-SSDLite & $\begin{array}{l}\text { GPU (Desktop) } \\
\text { GPU (Xavier) } \\
\text { CPU (Desktop) } \\
\text { CPU (Xavier) }\end{array}$ & 0.9398 & $\begin{array}{c}17.84 \\
85.54 \\
85.75 \\
5375.18\end{array}$ & $\begin{array}{l}- \\
- \\
- \\
-\end{array}$ & $\begin{array}{l}56.05 \\
11.69 \\
11.66 \\
0.186\end{array}$ & $\begin{array}{l}- \\
- \\
- \\
-\end{array}$ & $\begin{array}{c}126.7 \\
3.79 \\
99.0 \\
2.91\end{array}$ & $\begin{array}{c}0.442 \\
3.08 \\
0.117 \\
0.063\end{array}$ \\
\hline MobileNetV1-ConvLSTM-SSDLite $\left[\lambda_{0}=0.01\right]$ & $\begin{array}{l}\text { GPU (Desktop) } \\
\text { GPU (Xavier) } \\
\text { CPU (Desktop) } \\
\text { CPU (Xavier) }\end{array}$ & 0.9382 & $\begin{array}{c}17.89 \\
85.58 \\
85.81 \\
5354.33\end{array}$ & $\begin{array}{c}16.12 \\
70.78 \\
27.62 \\
3967.07\end{array}$ & $\begin{array}{c}55.91 \\
11.69 \\
11.68 \\
0.1869\end{array}$ & $\begin{array}{c}0.99 \\
1.0 \\
1.0 \\
1.0\end{array}$ & $\begin{array}{c}141.4 \\
4.19 \\
103.0 \\
3.15\end{array}$ & $\begin{array}{c}0.395 \\
2.83 \\
0.116 \\
0.061\end{array}$ \\
\hline MobileNetV1-ConvLSTM-SSDLite $\left[\lambda_{0}=0.5\right]$ & $\begin{array}{l}\text { GPU (Desktop) } \\
\text { GPU (Xavier) } \\
\text { CPU (Desktop) } \\
\text { CPU (Xavier) }\end{array}$ & 0.9377 & $\begin{array}{c}17.95 \\
84.77 \\
85.73 \\
5256.23\end{array}$ & $\begin{array}{c}16.38 \\
71.2 \\
26.94 \\
3845.12\end{array}$ & $\begin{array}{c}55.93 \\
11.88 \\
12.03 \\
0.1925\end{array}$ & $\begin{array}{l}0.99 \\
1.01 \\
1.03 \\
1.03\end{array}$ & $\begin{array}{c}141.4 \\
4.19 \\
103.0 \\
3.15\end{array}$ & $\begin{array}{c}0.395 \\
2.78 \\
0.116 \\
0.061\end{array}$ \\
\hline MobileNetV1-ConvLSTM-SSDLite $\left[\lambda_{0}=1.2\right]$ & $\begin{array}{l}\text { GPU (Desktop) } \\
\text { GPU (Xavier) } \\
\text { CPU (Desktop) } \\
\text { CPU (Xavier) }\end{array}$ & 0.8845 & $\begin{array}{c}17.96 \\
85.21 \\
85.9 \\
5312.47\end{array}$ & $\begin{array}{c}16.39 \\
71.04 \\
27.91 \\
3834.12\end{array}$ & $\begin{array}{c}58.41 \\
12.88 \\
18.25 \\
0.2212\end{array}$ & $\begin{array}{l}1.04 \\
1.1 \\
1.56 \\
1.18\end{array}$ & $\begin{array}{c}141.4 \\
4.19 \\
103.0 \\
3.15\end{array}$ & $\begin{array}{c}0.413 \\
3.07 \\
0.177 \\
0.07\end{array}$ \\
\hline MobileNetV1-ConvLSTM-SSDLite $\left[\lambda_{0}=2.0\right]$ & $\begin{array}{l}\text { GPU (Desktop) } \\
\text { GPU (Xavier) } \\
\text { CPU (Desktop) } \\
\text { CPU (Xavier) }\end{array}$ & 0.8403 & $\begin{array}{c}17.96 \\
86.58 \\
85.8 \\
5214.32\end{array}$ & $\begin{array}{c}16.68 \\
70.99 \\
26.74 \\
3956.01\end{array}$ & $\begin{array}{l}58.86 \\
13.37 \\
24.39 \\
0.234\end{array}$ & $\begin{array}{l}1.05 \\
1.14 \\
2.09 \\
1.25\end{array}$ & $\begin{array}{c}141.4 \\
4.19 \\
103.0 \\
3.15\end{array}$ & \begin{tabular}{c|c|}
0.416 \\
3.19 \\
0.236 \\
0.074
\end{tabular} \\
\hline
\end{tabular}

Imagenet VID 2015 dataset, whereas MobileNetV2 yielded higher $\mathrm{mAP}$ in the MAV-VID dataset.

The mAP gets reduced when the reward-conditional parameter $\lambda_{0}$ increases, since the policy is encouraged to execute more inattentional frames at the cost of accuracy. Nevertheless, it maintains competitive values throughout the whole range, with a minimum accuracy of 0.4373 and 0.8403 for Imagenet VID 2015 and MAV-VID dataset, respectively. As shown in Table 5 and 6, MobileNetV2-ConvLSTMSSDLite has remained as the model with lower mAP degradation.

The proposed inattentional framework has achieved a considerable latency reduction, increasing the runtime FPS in every platform tested, with minimal accuracy drop. 
TABLE 5. Results using the MobileNetV2 feature extractor on the ImageNet VID 2015 dataset. The mean Average Precision (mAP), KeyFrame (KF) and Inattentional Frame (IF) latency, as well as the average runtime FPS are provided. Also, the number of parameters of the models has been included. The FPS ratio with respect to the base FPS value (without the inattentional policy) has been also included for clarity. Additionally, power consumption and energy efficiency have been provided for every experiment.

\begin{tabular}{|c|c|c|c|c|c|c|c|c|c|}
\hline Model & Platform & $\mathrm{mAP}$ & $\begin{array}{c}\mathrm{KF} \\
(\mathrm{ms})\end{array}$ & $\begin{array}{c}\mathrm{IF} \\
(\mathrm{ms})\end{array}$ & FPS & $\begin{array}{l}\text { FPS } \\
\text { Ratio }\end{array}$ & $\begin{array}{c}\text { Params } \\
\text { (M) }\end{array}$ & $\begin{array}{c}\text { Power } \\
(\mathrm{W})\end{array}$ & $\begin{array}{c}\text { Efficiency } \\
\text { (FPS/W) }\end{array}$ \\
\hline MobileNetV2-SSDLite & $\begin{array}{l}\text { GPU (Desktop) } \\
\text { GPU (Xavier) } \\
\text { CPU (Desktop) } \\
\text { CPU (Xavier) }\end{array}$ & 0.4775 & $\begin{array}{c}35.79 \\
117.34 \\
297.88 \\
9001.32\end{array}$ & $\begin{array}{l}- \\
- \\
-\end{array}$ & $\begin{array}{c}27.94 \\
8.52 \\
3.35 \\
0.1111\end{array}$ & $\begin{array}{l}- \\
- \\
- \\
-\end{array}$ & 4.84 & $\begin{array}{l}127.3 \\
4.31 \\
98.2 \\
3.01\end{array}$ & $\begin{array}{c}0.219 \\
1.97 \\
0.034 \\
0.036\end{array}$ \\
\hline MobileNetV2-ConvLSTM-SSDLite & $\begin{array}{l}\text { GPU (Desktop) } \\
\text { GPU (Xavier) } \\
\text { CPU (Desktop) } \\
\text { CPU (Xavier) }\end{array}$ & 0.4924 & $\begin{array}{c}36.91 \\
126.82 \\
261.62 \\
8822.3 \\
\end{array}$ & $\begin{array}{l}- \\
- \\
- \\
-\end{array}$ & $\begin{array}{c}27.09 \\
7.7 \\
3.82 \\
0.1133\end{array}$ & $\begin{array}{l}- \\
- \\
- \\
-\end{array}$ & 3.3 & $\begin{array}{c}128.4 \\
5.03 \\
99.1 \\
3.12 \\
\end{array}$ & $\begin{array}{c}0.21 \\
1.53 \\
0.038 \\
0.036 \\
\end{array}$ \\
\hline MobileNetV2-ConvLSTM-SSDLite $\left[\lambda_{0}=0.01\right]$ & $\begin{array}{l}\text { GPU (Desktop) } \\
\text { GPU (Xavier) } \\
\text { CPU (Desktop) } \\
\text { CPU (Xavier) }\end{array}$ & 0.491 & $\begin{array}{c}35.64 \\
126.66 \\
263.126 \\
8852.42\end{array}$ & $\begin{array}{c}34.81 \\
118.53 \\
190.95 \\
6267.98\end{array}$ & $\begin{array}{c}28.14 \\
7.96 \\
3.94 \\
0.1176\end{array}$ & $\begin{array}{l}1.03 \\
1.03 \\
1.03 \\
1.03\end{array}$ & 5.5 & $\begin{array}{c}142.5 \\
5.17 \\
102.9 \\
3.23\end{array}$ & $\begin{array}{c}0.197 \\
1.53 \\
0.038 \\
0.036 \\
\end{array}$ \\
\hline MobileNetV2-ConvLSTM-SSDLite $\left[\lambda_{0}=0.5\right]$ & $\begin{array}{l}\text { GPU (Desktop) } \\
\text { GPU (Xavier) } \\
\text { CPU (Desktop) } \\
\text { CPU (Xavier) }\end{array}$ & 0.4818 & $\begin{array}{c}36.83 \\
126.32 \\
264.02 \\
8752.36\end{array}$ & $\begin{array}{c}34.39 \\
118.79 \\
163.84 \\
6189.12\end{array}$ & $\begin{array}{c}28.03 \\
8.14 \\
4.62 \\
0.1312\end{array}$ & $\begin{array}{c}1.03 \\
1.05 \\
1.2 \\
1.15\end{array}$ & 5.5 & $\begin{array}{c}142.5 \\
5.17 \\
102.9 \\
3.23\end{array}$ & $\begin{array}{c}0.196 \\
1.57 \\
0.044 \\
0.04\end{array}$ \\
\hline MobileNetV2-ConvLSTM-SSDLite $\left[\lambda_{0}=1.2\right]$ & $\begin{array}{l}\text { GPU (Desktop) } \\
\text { GPU (Xavier) } \\
\text { CPU (Desktop) } \\
\text { CPU (Xavier) }\end{array}$ & 0.4704 & $\begin{array}{c}36.67 \\
126.08 \\
264.14 \\
8952.78\end{array}$ & $\begin{array}{c}34.71 \\
119.1 \\
152.34 \\
6104.78\end{array}$ & $\begin{array}{c}28.24 \\
8.22 \\
5.2 \\
0.1406\end{array}$ & $\begin{array}{l}1.04 \\
1.06 \\
1.36 \\
1.24\end{array}$ & 5.5 & $\begin{array}{c}142.5 \\
5.17 \\
102.9 \\
3.23\end{array}$ & $\begin{array}{c}0.198 \\
1.58 \\
0.05 \\
0.043\end{array}$ \\
\hline MobileNetV2-ConvLSTM-SSDLite $\left[\lambda_{0}=2.0\right]$ & $\begin{array}{l}\text { GPU (Desktop) } \\
\text { GPU (Xavier) } \\
\text { CPU (Desktop) } \\
\text { CPU (Xavier) }\end{array}$ & 0.4622 & $\begin{array}{c}36.08 \\
126.25 \\
264.22 \\
8876.23\end{array}$ & $\begin{array}{c}34.19 \\
118.68 \\
144.99 \\
6123.43\end{array}$ & $\begin{array}{c}28.89 \\
8.3 \\
5.83 \\
0.1485\end{array}$ & $\begin{array}{l}1.06 \\
1.07 \\
1.52 \\
1.31\end{array}$ & 5.5 & $\begin{array}{c}142.5 \\
5.17 \\
102.9 \\
3.23\end{array}$ & \begin{tabular}{c|c|}
0.202 \\
1.6 \\
0.056 \\
0.045
\end{tabular} \\
\hline
\end{tabular}

TABLE 6. Results using the MobileNetV2 feature extractor on the MAV-VID dataset. The mean Average Precision (mAP), KeyFrame (KF) and Inattentional Frame (IF) latency, as well as the average runtime FPS are provided. The FPS ratio with respect to the base FPS value (without the inattentional policy) has been also included for clarity. Additionally, power consumption and energy efficiency have been provided for every experiment.

\begin{tabular}{|c|c|c|c|c|c|c|c|c|}
\hline Model & Platform & mAP & $\begin{array}{c}\mathrm{KF} \\
(\mathrm{ms})\end{array}$ & $\begin{array}{c}\text { IF } \\
(\mathrm{ms})\end{array}$ & FPS & $\begin{array}{l}\text { FPS } \\
\text { Ratio }\end{array}$ & $\begin{array}{c}\text { Power } \\
(\mathrm{W})\end{array}$ & $\begin{array}{l}\text { Efficiency } \\
\text { (FPS/W) }\end{array}$ \\
\hline \multirow{4}{*}{ MobileNetV2-SSDLite } & GPU (Desktop) & \multirow{4}{*}{0.9218} & 25.819 & - & 38.73 & - & 127.4 & 0.304 \\
\hline & GPU (Xavier) & & 120.82 & - & 8.27 & - & 4.32 & 1.91 \\
\hline & CPU (Desktop) & & 222.92 & - & 4.48 & - & 98.1 & 0.045 \\
\hline & CPU (Xavier) & & 7645.68 & - & 0.1308 & - & 3.16 & 0.041 \\
\hline \multirow{3}{*}{ MobileNetV2-ConvLSTM-SSDLite } & GPU (Desktop) & \multirow{3}{*}{0.9453} & 29.78 & - & 33.57 & - & 128.5 & 0.261 \\
\hline & GPU (Xavier) & & 123.15 & - & 8.12 & - & 5.14 & 1.57 \\
\hline & CPU (Xavier) & & 8002.68 & - & 0.125 & - & 3.13 & 0.039 \\
\hline \multirow{4}{*}{ MobileNetV2-ConvLSTM-SSDLite $\left[\lambda_{0}=0.01\right]$} & GPU (Desktop) & \multirow{4}{*}{0.9453} & 29.2 & - & 34.24 & 1.02 & 142.6 & 0.24 \\
\hline & GPU (Xavier) & & 123.02 & - & 8.12 & 1.0 & 5.11 & 1.58 \\
\hline & CPU (Desktop) & & 231.25 & - & 4.32 & 1.0 & 102.8 & 0.042 \\
\hline & CPU (Xavier) & & 7903.04 & - & 0.1265 & 1.0 & 3.13 & 0.04 \\
\hline \multirow{3}{*}{ MobileNetV2-ConvLSTM-SSDLite $\left[\lambda_{0}=0.5\right]$} & GPU (Desktop) & \multirow{3}{*}{0.9452} & 29.13 & 28.14 & 34.33 & 1.02 & 142.6 & 0.24 \\
\hline & GPU (Xavier) & & 122.47 & 115.23 & 8.16 & 1.0 & 5.11 & 1.59 \\
\hline & CPU (Desktop) & & 230.91 & 50.75 & 4.33 & 1.0 & 102.8 & 0.042 \\
\hline \multirow{4}{*}{ MobileNetV2-ConvLSTM-SSDLite $\left[\lambda_{0}=1.2\right]$} & GPU (Desktop) & \multirow{4}{*}{0.86} & 29.08 & 28.47 & 34.78 & 1.03 & 142.6 & 0.243 \\
\hline & GPU (Xavier) & & 123.11 & 115.1 & 8.41 & 1.03 & 5.11 & 1.64 \\
\hline & CPU (Desktop) & & 232.09 & 51.77 & 7.43 & 1.71 & 102.8 & 0.07 \\
\hline & CPU (Xavier) & & 8009.22 & 3243.22 & 0.1842 & 1.47 & 3.13 & 0.058 \\
\hline \multirow{4}{*}{ MobileNetV2-ConvLSTM-SSDLite $\left[\lambda_{0}=2.0\right]$} & GPU (Desktop) & \multirow{4}{*}{0.8364} & 28.88 & 28.49 & 34.93 & 1.04 & 142.6 & 0.244 \\
\hline & GPU (Xavier) & & 123.15 & 114.65 & 8.51 & 1.04 & 5.11 & 1.66 \\
\hline & CPU (Desktop) & & 232.04 & 51.22 & 8.92 & 2.06 & 102.8 & 0.086 \\
\hline & CPU (Xavier) & & 7865.12 & 3500.33 & 0.2013 & 1.61 & 3.13 & 0.064 \\
\hline
\end{tabular}

Nevertheless, the amount of computation reduction is highly dependent on the platform where the system is executed, as well as on the average object size within the image plane. Considering the GPU platforms, with a higher parallelization capacity, the average FPS increase ratio has ranged from 1.0 to 1.14 for MobileNetV1 and from 1.0 to 1.07 for MobileNetV2. However, regarding the CPU platforms, where the parallelization capacity is limited, the average FPS increase ratio has ranged from 1.0 to 2.09 for MobileNetV1 and from 1.0 to 2.06 for MobileNetV2. These results lead to an average FPS on the desktop CPU platform of 37.73 , which is in the order of magnitude of the base runtime 

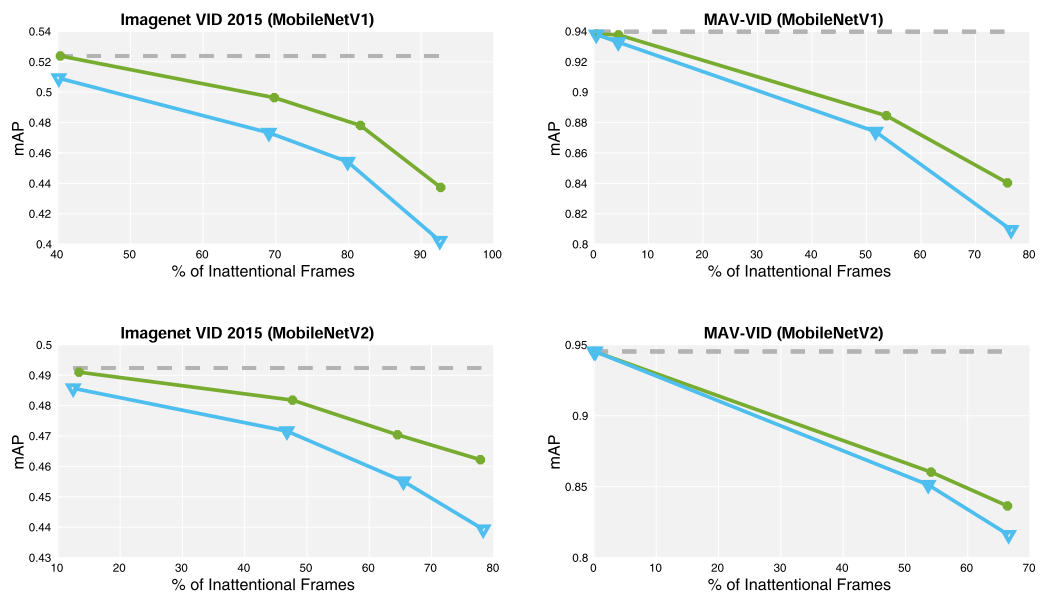

FIGURE 5. Representation of the trade-off between the mAP and the percentage of inattentional frames executed. Four cases with two feature extractors (MobileNetV1 and MobileNetV2) and two datasets (Imagenet VID 2015 and MAV-VID) have been evaluated. The random baseline policy is depicted in pale blue (circles) and the proposed inattentional policy in pale green (triangles). The original mAP (with no inattentional frames involved) is represented with a grey dashed line. The inattentional policy predominates allover the tests, resulting in a higher mAP for every percentage of inattentional frames. Also, the inattentional policy incurs less accuracy degradation even at extreme ratios of inattentional frames, suggesting that our method is superior at capturing the temporal dynamics inherent to videos. Every test has been performed 5 times each, for both policies, and the average results have been plotted.

FPS on the desktop GPU platform of 51.91, at the cost of $0.086 \mathrm{mAP}$ reduction, for MobileNetV1 in Imagenet VID 2015 dataset. The maximum FPS increase ratio has been 2.09 for MobileNetV1 on the desktop CPU, achieving a runtime FPS of 24.39 at the cost of $0.09 \mathrm{mAP}$ reduction, in MAV-VID dataset (where the object size is on average smaller in the image plane). These results suggest that the inattentional framework is able to increase computation efficiency when the effective parallelization capacity is limited. The effective parallelization capacity is influenced by several variables and it is relative to the input images, the model size and the platform specifications. Qualitatively, parallelization is low in average CPUs, in applications where the input images are at high resolution or in applications where the models have notably more parameters than our MobileNetV1/V2-ConvLSTM-SSDLite models. These facts yield to an open field of research, where the proposed inattentional framework can be applied. Nevertheless, such extensive study is out of the scope of the present work.

Regarding power consumption and energy efficiency, the proposed inattentional framework provides proper results in terms of both global power consumption and energy efficiency increase. Power consumption is notably higher in desktop GPU/CPUs, with on average $35 \times$ more power consumption than the embedded Xavier platform. The lowest energy consumption has been $2.87 \mathrm{~W}$ for MobileNetV1-ConvLSTM-SSDLite base model (no inattentional policy) in Imagenet VID 2015 dataset. Energy efficiency varies across platforms, being the GPUs more energy efficient due to their parallelization capabilities. The maximum energy efficiency has resulted in 3.19 FPS/W $(1.12 \times)$ for MobileNetV1 on the Xavier GPU, achieving a runtime FPS of 13.37 at the cost of $4.19 \mathrm{~W}$, in MAV-VID dataset. It has to be noted that, regarding relative energy efficiency increase with respect to the base energy efficiency (no inattentional frames), the results follows approximately the same ratios as in the FPS case, resulting in a maximum energy efficiency increase of $2.09 \times$ for MobileNetV1 on the desktop CPU, and achieving a runtime FPS of 24.39 at the cost of $0.09 \mathrm{mAP}$ reduction, in MAV-VID dataset.

Considering Fig. 5, in comparison to the random baseline, our inattentional policy predominates allover the tests, providing a higher mAP for every percentage of inattentional frames executed. Furthermore, our inattentional policy shows lower mAP degradation even at extreme percentage of inattentional frames, suggesting that our method is superior at capturing the temporal dynamics inherent to videos. The highest mAP distance to the random baseline has been provided by the MobileNetV1-ConvLSTM-SSDLite in the Imagenet VID 2015 dataset, with a distance of 0.4 in mAP at $92 \%$ of inattentional frames executed. Another emergent property is that, for the case of MobileNetV1 in Imagenet VID 2015 dataset, the inattentional policy is able to match the original mAP even when executing a $40 \%$ of inattentional frames, which suggests there is a redundancy of information that does not add value to the final accuracy. In addition, thanks to our novel reward-conditional training scheme, the policy can be conditioned at inference time, providing 


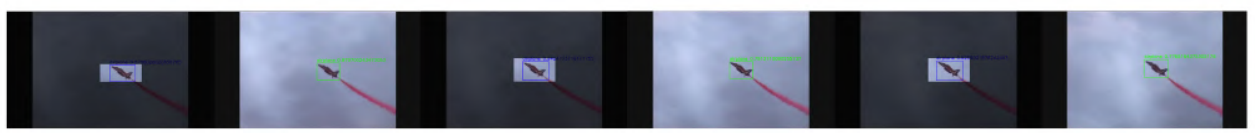

(a)

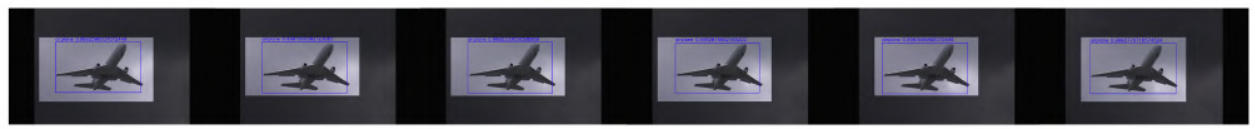

(b)

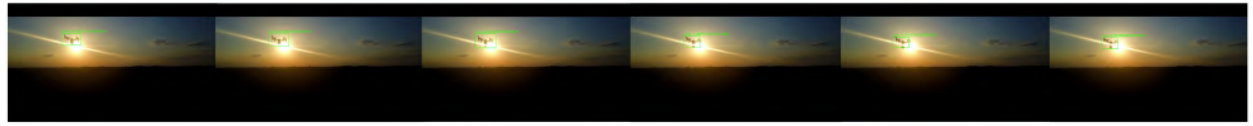

(c)

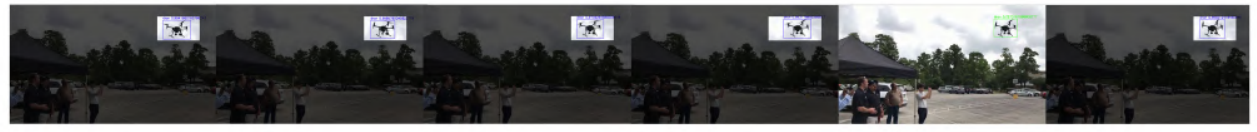

(d)

FIGURE 6. Images corresponding to four different scenarios for both datasets. Every image example has been processed by MobileNetV1-ConvLSTM-SSDLite $\left[\lambda_{0}=1.2\right]$ model (MobileNetV2-ConvLSTM-SSDLite provides a similar behavior). Shaded regions correspond to non-processed pixels. (a)-(b) and (c)-(d) image examples correspond to Imagenet VID 2015 and MAV-VID dataset, respectively. (a) and (c) show complex detection examples, corresponding to an object with high-motion speed within the image plane, and to an increased environmental complexity (camera pointing to the sun), respectively. In this context, the inattentional policy executes a lower percentage of inattentional frames. (b) and (d) illustrate objects which remain more static throughout the sequence. In this scenario, the number of inattentional frames is higher.

promising results in a wide variety of sequences with varying backgrounds, changes in illumination, object size, etc. and allowing for real-time performance modulation in the context of the required application (see supplementary video ${ }^{2}$ ).

Finally, at the cost of $2.2 \mathrm{M}$ parameters, a learned inattentional policy can provide adaptiveness to the video dynamics, as shown in Fig. 6. In this figure, two complex and two simple scenarios, in terms of detection difficultness, are illustrated. In the complex scenarios, where the object is moving fast within the image plane, or there are ambient conditions which difficult detection, the inattentional policy performs a higher rate of full keyframes in order to maintain the detection accuracy of the object through the sequence. Nevertheless, when the scenarios are simpler, such as the case an object with slow motion in the image plane, the inattentional policy tends to neglect context to speedup computation, without missing accuracy. This adaptive behavior can be very promising in a wide variety of video object detection applications.

\section{CONCLUSION}

In this work, the inattentional framework has been studied. The presented framework aims at reducing the computation overhead, in the frame of video object detection, by reusing redundant context in video images. An inattentional policy has been learned, under the reinforcement learning paradigm, to select the amount of frames where the context is reused.

\footnotetext{
${ }^{2}$ https://vimeo.com/426725929
}

Furthermore, a novel reward-conditional training has been presented, where a policy can be trained on a distribution of reward functions and conditioned on one unique function at inference time. The inattentional framework provided an average latency reduction in CPUs up to 2.09 times the original latency, and obtaining FPS rates similar to their equivalent GPU platform, at the cost of a mAP reduction of 1.11 times.

This study could be extended by evaluating the inattentional framework in other scenarios of low parallelization capacity, such as the case of high resolution input images or mobile devices. Also, optimization techniques and shallower architectures can be further applied, such as half-precision inference, quantization or MobileNets with $\alpha<1$. Regarding the reward-conditional training, a complete study of this method with a diverse set of reward functions and reinforcement learning algorithms has been left as future work.

\section{SUPPLEMENTARY MATERIAL}

The code has been made publicly available at https:// github.com/alejodosr/adaptive-inattention. Also, a short video demonstration can be found at https://vimeo.com/ 426725929. The MAV-VID dataset can be downloaded at https://www.kaggle.com/alejodosr/multirotoraerial-vehiclevid-mavvid-dataset.

\section{ACKNOWLEDGMENT}

The authors would like to acknowledge Estefanía Carolina Asimbaya Shuguli for the exhaustive annotations of the images for the generation of the public dataset. 


\section{REFERENCES}

[1] A. Bochkovskiy, C.-Y. Wang, and H.-Y. Mark Liao, "YOLOv4: Optimal speed and accuracy of object detection," 2020, arXiv:2004.10934 [Online]. Available: http://arxiv.org/abs/2004.10934

[2] S. Liu, D. Huang, and Y. Wang, "Learning spatial fusion for singleshot object detection," 2019, arXiv:1911.09516. [Online]. Available: http://arxiv.org/abs/1911.09516

[3] T.-Y. Lin, P. Goyal, R. Girshick, K. He, and P. Dollár, "Focal loss for dense object detection," in Proc. IEEE Int. Conf. Comput. Vis., Oct. 2017, pp. 2980-2988.

[4] M. Tan, R. Pang, and Q. V. Le, "EfficientDet: Scalable and efficient object detection," 2019, arXiv:1911.09070. [Online]. Available: http://arxiv.org/abs/1911.09070

[5] X. Zhou, D. Wang, and P. Krähenbühl, "Objects as points," 2019, arXiv:1904.07850. [Online]. Available: http://arxiv.org/abs/1904.07850

[6] J. Redmon and A. Farhadi, "YOLOv3: An incremental improvement," 2018, arXiv:1804.02767. [Online]. Available: http://arxiv.org/abs/ 1804.02767

[7] R. Girshick, "Fast R-CNN," in Proc. IEEE Int. Conf. Comput. Vis., Dec. 2015, pp. 1440-1448.

[8] R. Huang, J. Pedoeem, and C. Chen, "YOLO-LITE: A real-time object detection algorithm optimized for non-GPU computers," in Proc. IEEE Int. Conf. Big Data (Big Data), Dec. 2018, pp. 2503-2510.

[9] M. Gao, R. Yu, A. Li, V. I. Morariu, and L. S. Davis, "Dynamic zoomin network for fast object detection in large images," in Proc. IEEE Conf Comput. Vis. Pattern Recognit., Jun. 2018, pp. 6926-6935.

[10] A. Pirinen and C. Sminchisescu, "Deep reinforcement learning of region proposal networks for object detection," in Proc. IEEE Conf. Comput. Vis. Pattern Recognit., Jun. 2018, pp. 6945-6954.

[11] K. Rungsuptaweekoon, V. Visoottiviseth, and R. Takano, "Evaluating the power efficiency of deep learning inference on embedded GPU systems," in Proc. 2nd Int. Conf. Inf. Technol. (INCIT), Nov. 2017, pp. 1-5.

[12] H. Mao, S. Yao, T. Tang, B. Li, J. Yao, and Y. Wang, "Towards realtime object detection on embedded systems," IEEE Trans. Emerg. Topics Comput., vol. 6, no. 3, pp. 417-431, Sep. 2018.

[13] J. Yu, K. Guo, Y. Hu, X. Ning, J. Qiu, H. Mao, S. Yao, T. Tang, B. Li, Y. Wang, and H. Yang, "Real-time object detection towards high power efficiency," in Proc. Design, Autom. Test Eur. Conf. Exhib. (DATE), Mar. 2018, pp. 704-708.

[14] F. N. Iandola, S. Han, M. W. Moskewicz, K. Ashraf, W. J. Dally, and K. Keutzer, "SqueezeNet: AlexNet-level accuracy with 50x fewer parameters and <0.5 MB model size," 2016, arXiv:1602.07360. [Online]. Available: http://arxiv.org/abs/1602.07360

[15] A. Howard, M. Sandler, B. Chen, W. Wang, L.-C. Chen, M. Tan, G. Chu, V. Vasudevan, Y. Zhu, R. Pang, H. Adam, and Q. Le, "Searching for MobileNetV3," in Proc. IEEE Int. Conf. Comput. Vis., Oct. 2019, pp. 1314-1324.

[16] A. G. Howard, M. Zhu, B. Chen, D. Kalenichenko, W. Wang, T. Weyand M. Andreetto, and H. Adam, "MobileNets: Efficient convolutional neural networks for mobile vision applications," 2017, arXiv:1704.04861 [Online]. Available: http://arxiv.org/abs/1704.04861

[17] M. Sandler, A. Howard, M. Zhu, A. Zhmoginov, and L.-C. Chen, "MobileNetV2: Inverted residuals and linear bottlenecks," in Proc. IEEE Conf. Comput. Vis. Pattern Recognit., Jun. 2018, pp. 4510-4520.

[18] M. Tan, B. Chen, R. Pang, V. Vasudevan, and Q. V. Le, "MnasNet Platform-aware neural architecture search for mobile," in Proc. IEEE/CVF Conf. Comput. Vis. Pattern Recognit. (CVPR), Jun. 2018, pp. 2815-2823.

[19] X. Zhang, X. Zhou, M. Lin, and J. Sun, "ShuffleNet: An extremely efficient convolutional neural network for mobile devices," in Proc. IEEE Conf. Comput. Vis. Pattern Recognit., Jun. 2018, pp. 6848-6856.

[20] N. Ma, X. Zhang, H.-T. Zheng, and J. Sun, "ShuffleNet V2: Practical guidelines for efficient $\mathrm{CNN}$ architecture design," in Proc. Eur. Conf. Comput. Vis. (ECCV), 2018, pp. 116-131.

[21] I. Hubara, M. Courbariaux, D. Soudry, R. El-Yaniv, and Y. Bengio, "Binarized neural networks," in Proc. Adv. Neural Inf. Process. Syst., 2016, pp. 4107-4115.

[22] I. Hubara, M. Courbariaux, D. Soudry, R. El-Yaniv, and Y. Bengio, "Quantized neural networks: Training neural networks with low precision weights and activations," J. Mach. Learn. Res., vol. 18, no. 1, pp. 6869-6898, 2017.

[23] P. Bacchus, R. Stewart, and E. Komendantskaya, "Accuracy, training time and hardware efficiency trade-offs for quantized neural networks on FPGAs," in Proc. Int. Symp. Appl. Reconfigurable Comput. Toledo, Spain: Springer, 2020, pp. 121-135.
[24] C. Ding, S. Wang, N. Liu, K. Xu, Y. Wang, and Y. Liang, "REQ-YOLO: A resource-aware, efficient quantization framework for object detection on FPGAs," in Proc. ACM/SIGDA Int. Symp. Field-Programmable Gate Arrays, Feb. 2019, pp. 33-42.

[25] Y. Kim, M. Imani, and T. S. Rosing, "Image recognition accelerator design using in-memory processing," IEEE Micro, vol. 39, no. 1, pp. 17-23, Jan. 2019.

[26] J. Aloimonos, I. Weiss, and A. Bandyopadhyay, "Active vision," Int. J. Comput. Vis., vol. 1, no. 4, pp. 333-356, 1988.

[27] J. M. Findlay and I. D. Gilchrist, Active Vision: The Psychology of Looking and Seeing, no. 37. London, U.K.: Oxford Univ. Press, 2003.

[28] H. Kolb, "Simple anatomy of the retina," in Webvision: The Organization of the Retina and Visual System. Salt Lake City, UT, USA: The Organization of the Retina and Visual System, 1995, pp. 13-36.

[29] H. Strasburger, I. Rentschler, and M. Jüttner, "Peripheral vision and pattern recognition: A review," J. Vis., vol. 11, no. 5, p. 13, 2011.

[30] W. Kienzle, M. O. Franz, B. Schölkopf, and F. A. Wichmann, "Centersurround patterns emerge as optimal predictors for human saccade targets," J. Vis., vol. 9, no. 5, p. 7, May 2009.

[31] D. Purves, G. J. Augustine, D. Fitzpatrick, W. Hall, A.-S. LaMantia, J. O. McNamara, and L. White, Neuroscience. Sunderland, MA, USA: Sinauer Associates, 2001.

[32] A. Mack and I. Rock, Inattentional Blindness. Cambridge, MA, USA: MIT Press, 1998.

[33] J. Li, F. Shi, W. Liu, D. Zou, Q. Wang, P.-K. J. Park, and H. E. Ryu, "Adaptive Temporal Pooling for Object Detection using Dynamic Vision Sensor," in Proc. Brit. Mach. Vis. Conf. (BMVC), T.-K. Kim, S. Zafeiriou, G. Brostow, and K. Mikolajczyk, Eds. BMVA Press, Sep. 2017, pp. 40.1-40.12. [Online]. Available: https://dx.doi.org/10.5244/C.31.40, doi: $10.5244 /$ C. 31.40

[34] N. F. Y. Chen, "Pseudo-labels for supervised learning on dynamic vision sensor data, applied to object detection under ego-motion," in Proc. IEEE Conf. Comput. Vis. Pattern Recognit. Workshops, Jun. 2018, pp. 644-653.

[35] A. Dosovitskiy and J. Djolonga, "You only train once: Loss-conditional training of deep networks," in Proc. Int. Conf. Learn. Represent., 2020.

[36] E. Perez, F. Strub, H. D. Vries, V. Dumoulin, and A. Courville, "Film: Visual reasoning with a general conditioning layer," in Proc. 32nd AAAI Conf. Artif. Intell., 2018, pp. 3942-3951.

[37] J. Schulman, F. Wolski, P. Dhariwal, A. Radford, and O. Klimov, "Proximal policy optimization algorithms," 2017, arXiv:1707.06347. [Online]. Available: http://arxiv.org/abs/1707.06347

[38] T. P. Lillicrap, J. J. Hunt, A. Pritzel, N. Heess, T. Erez, Y. Tassa, D. Silver, and D. Wierstra, "Continuous control with deep reinforcement learning," 2015, arXiv:1509.02971. [Online]. Available: http://arxiv.org/abs/1509.02971

[39] J. Schulman, S. Levine, P. Abbeel, M. Jordan, and P. Moritz, "Trust region policy optimization," in Proc. Int. Conf. Mach. Learn., 2015, pp. 1889-1897.

[40] X. Zhu, Y. Xiong, J. Dai, L. Yuan, and Y. Wei, "Deep feature flow for video recognition," in Proc. IEEE Conf. Comput. Vis. Pattern Recognit., Jul. 2017, pp. 2349-2358

[41] X. Zhu, Y. Wang, J. Dai, L. Yuan, and Y. Wei, "Flow-guided feature aggregation for video object detection," in Proc. IEEE Int. Conf. Comput. Vis., Oct. 2017, pp. 408-417.

[42] C. Hetang, H. Qin, S. Liu, and J. Yan, "Impression network for video object detection," 2017, arXiv:1712.05896. [Online]. Available: http://arxiv.org/abs/1712.05896

[43] X. Zhu, J. Dai, X. Zhu, Y. Wei, and L. Yuan, "Towards high performance video object detection for mobiles," 2018, arXiv:1804.05830. [Online]. Available: http://arxiv.org/abs/1804.05830

[44] Z. Zhu, W. Wu, W. Zou, and J. Yan, "End-to-end flow correlation tracking with spatial-temporal attention," in Proc. IEEE Conf. Comput. Vis. Pattern Recognit., Jun. 2018, pp. 548-557.

[45] L. Zhang, Z. Lin, J. Zhang, H. Lu, and Y. He, "Fast video object segmentation via dynamic targeting network," in Proc. IEEE Int. Conf. Comput. Vis., Oct. 2019, pp. 5582-5591.

[46] S. Jain, X. Wang, and J. E. Gonzalez, "Accel: A corrective fusion network for efficient semantic segmentation on video," in Proc. IEEE Conf. Comput. Vis. Pattern Recognit. (CVPR), Jun. 2019, pp. 8866-8875.

[47] B. Mahasseni, S. Todorovic, and A. Fern, "Budget-aware deep semantic video segmentation," in Proc. IEEE Conf. Comput. Vis. Pattern Recognit., Jul. 2017, pp. 1029-1038.

[48] F. Xiao and Y. J. Lee, "Video object detection with an aligned spatialtemporal memory," in Proc. Eur. Conf. Comput. Vis. (ECCV), pp. 485-501, 2018. 
[49] M. Liu, M. Zhu, M. White, Y. Li, and D. Kalenichenko, "Looking fast and slow: Memory-guided mobile video object detection," 2019, arXiv:1903.10172. [Online]. Available: http://arxiv.org/abs/1903.10172

[50] M. Zhu and M. Liu, "Mobile video object detection with temporallyaware feature maps," in Proc. IEEE Conf. Comput. Vis. Pattern Recognit., Jun. 2018, pp. 5686-5695

[51] X. Chen, J. Yu, and Z. Wu, "Temporally identity-aware SSD with attentional LSTM," IEEE Trans. Cybern., vol. 50, no. 6, pp. 2674-2686, Jun. 2020

[52] S. S. Nabavi, M. Rochan, and W. Yang, "Future semantic segmentation with convolutional LSTM,” 2018, arXiv:1807.07946. [Online]. Available: http://arxiv.org/abs/1807.07946

[53] O. Russakovsky, J. Deng, H. Su, J. Krause, S. Satheesh, S. Ma, Z. Huang, A. Karpathy, A. Khosla, M. Bernstein, A. C. Berg, and L. Fei-Fei, "ImageNet large scale visual recognition challenge," Int. J. Comput. Vis., vol. 115 , no. 3, pp. 211-252, Dec. 2015.

[54] W. Han, P. Khorrami, T. Le Paine, P. Ramachandran, M. Babaeizadeh, H. Shi, J. Li, S. Yan, and T. S. Huang, "Seq-NMS for video object detection," 2016, arXiv:1602.08465. [Online]. Available: http://arxiv. org/abs/1602.08465

[55] K. Kang, H. Li, J. Yan, X. Zeng, B. Yang, T. Xiao, C. Zhang, Z. Wang, R. Wang, X. Wang, and W. Ouyang, "T-CNN: Tubelets with convolutional neural networks for object detection from videos," IEEE Trans. Circuits Syst. Video Technol., vol. 28, no. 10, pp. 2896-2907, Oct. 2018.

[56] K. Kang, W. Ouyang, H. Li, and X. Wang, "Object detection from video tubelets with convolutional neural networks," in Proc. IEEE Conf. Comput. Vis. Pattern Recognit., Jun. 2016, pp. 817-825.

[57] H. Deng, Y. Hua, T. Song, Z. Zhang, Z. Xue, R. Ma, N. Robertson, and H. Guan, "Object guided external memory network for video object detection," in Proc. IEEE Int. Conf. Comput. Vis., Oct. 2019, pp. 6678-6687.

[58] L. Yang, Y. Fan, and N. Xu, "Video instance segmentation," in Proc. IEEE Int. Conf. Comput. Vis., Oct. 2019, pp. 5188-5197.

[59] H. Mao, T. Kong, and W. J. Dally, "CaTDet: Cascaded tracked detector fo efficient object detection from video," 2018, arXiv:1810.00434. [Online] Available: http://arxiv.org/abs/1810.00434

[60] H. Luo, W. Xie, X. Wang, and W. Zeng, "Detect or track: Towards cost-effective video object detection/tracking," in Proc. AAAI Conf. Artif. Intell., vol. 33, 2019, pp. 8803-8810.

[61] C. Feichtenhofer, A. Pinz, and A. Zisserman, "Detect to track and track to detect," in Proc. IEEE Int. Conf. Comput. Vis., Oct. 2017, pp. 3038-3046.

[62] Z. Jiang, P. Gao, C. Guo, Q. Zhang, S. Xiang, and C. Pan, "Video object detection with locally-weighted deformable neighbors," in Proc. AAAI Conf. Artif. Intell., vol. 33, 2019, pp. 8529-8536.

[63] G. Bertasius, L. Torresani, and J. Shi, "Object detection in video with spatiotemporal sampling networks," in Proc. Eur. Conf. Comput. Vis. (ECCV), 2018, pp. 331-346.

[64] K. Chen, J. Wang, S. Yang, X. Zhang, Y. Xiong, C. C. Loy, and D. Lin "Optimizing video object detection via a scale-time lattice," in Proc. IEEE Conf. Comput. Vis. Pattern Recognit., Jun. 2018, pp. 7814-7823.

[65] Y. Li, J. Shi, and D. Lin, "Low-latency video semantic segmentation," in Proc. IEEE Conf. Comput. Vis. Pattern Recognit., Jun. 2018, pp. 5997-6005

[66] C. Ying and K. Fragkiadaki, "Depth-adaptive computational policies for efficient visual tracking," in Proc. Int. Workshop Energy Minimization Methods Comput. Vis. Pattern Recognit. Venice, Italy: Springer, 2017, pp. $109-122$

[67] J. Supancic and D. Ramanan, "Tracking as online decision-making: Learning a policy from streaming videos with reinforcement learning," in Proc. IEEE Int. Conf. Comput. Vis., Oct. 2017, pp. 322-331.

[68] X. Hou and L. Zhang, "Saliency detection: A spectral residual approach," in Proc. IEEE Conf. Comput. Vis. Pattern Recognit., Jun. 2007, pp. 1-8.

[69] L. Itti, C. Koch, and E. Niebur, "A model of saliency-based visual attention for rapid scene analysis," IEEE Trans. Pattern Anal. Mach. Intell., vol. 20, no. 11 , pp. $1254-1259,1998$.

[70] M. Najibi, B. Singh, and L. Davis, "AutoFocus: Efficient multi-scale inference," in Proc. IEEE Int. Conf. Comput. Vis., Oct. 2019, pp. 9745-9755.

[71] C. Chen, M.-Y. Liu, O. Tuzel, and J. Xiao, "R-CNN for small object detection," in Proc. Asian Conf. Comput. Vis. Taipei, Taiwan: Springer, 2016, pp. 214-230.

[72] Y. Lu, T. Javidi, and S. Lazebnik, "Adaptive object detection using adjacency and zoom prediction," in Proc. IEEE Conf. Comput. Vis. Pattern Recognit., Jun. 2016, pp. 2351-2359.

[73] S. Gidaris and N. Komodakis, "Object detection via a multi-region and semantic segmentation-aware CNN model," in Proc. IEEE Int. Conf. Comput. Vis., Dec. 2015, pp. 1134-1142.
[74] Y. Zhu, R. Urtasun, R. Salakhutdinov, and S. Fidler, "SegDeepM: Exploiting segmentation and context in deep neural networks for object detection," in Proc. IEEE Conf. Comput. Vis. Pattern Recognit., Jun. 2015, pp. 4703-4711

[75] J. Ba, V. Mnih, and K. Kavukcuoglu, "Multiple object recognition with visual attention," 2014, arXiv:1412.7755. [Online]. Available: http://arxiv.org/abs/1412.7755

[76] S. Mathe, A. Pirinen, and C. Sminchisescu, "Reinforcement learning for visual object detection," in Proc. IEEE Conf. Comput. Vis. Pattern Recognit., Jun. 2016, pp. 2894-2902.

[77] J. C. Caicedo and S. Lazebnik, "Active object localization with deep reinforcement learning," in Proc. IEEE Int. Conf. Comput. Vis., Dec. 2015, pp. $2488-2496$

[78] B. Uzkent and S. Ermon, "Learning when and where to zoom with deep reinforcement learning," 2020, arXiv:2003.00425. [Online]. Available: http://arxiv.org/abs/2003.00425

[79] B. Uzkent, C. Yeh, and S. Ermon, "Efficient object detection in large images using deep reinforcement learning," in Proc. IEEE Winter Conf. Appl. Comput. Vis., Mar. 2020, pp. 1824-1833.

[80] Z. Jie, X. Liang, J. Feng, X. Jin, W. Lu, and S. Yan, "Tree-structured reinforcement learning for sequential object localization," in Proc. Adv Neural Inf. Process. Syst., 2016, pp. 127-135.

[81] W. Liu, D. Anguelov, D. Erhan, C. Szegedy, S. Reed, C.-Y. Fu, and A. C. Berg, "SSD: Single shot multibox detector," in Proc. Eur. Conf. Comput. Vis. Amsterdam, The Netherlands: Springer, 2016, pp. 21-37.

[82] K. He, X. Zhang, S. Ren, and J. Sun, "Deep residual learning for image recognition," in Proc. IEEE Conf. Comput. Vis. Pattern Recognit., Jun. 2016, pp. 770-778.

[83] A. Paszke, S. Gross, F. Massa, A. Lerer, J. Bradbury, G. Chanan, T. Killeen, Z. Lin, N. Gimelshein L. Antiga, and A. Desmaison, "PyTorch: An imperative style, high-performance deep learning library," in Proc. Adv. Neural Inf. Process. Syst., 2019, pp. 8024-8035.

[84] M. Abadi et al., "TensorFlow: A system for large-scale machine learning," in Proc. 12th USENIX Symp. Oper. Syst. Design Implement. (OSDI), 2016, pp. $265-283$.

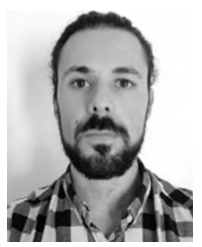

ALEJANDRO RODRIGUEZ-RAMOS (Graduate Student Member, IEEE) received the M.Sc. degree in telecommunication engineering (major in electronics and micro-electronics) from the Universidad Politécnica de Madrid (UPM), Madrid, Spain, in 2015, where he is currently pursuing the Ph.D. degree with the Centre for Automation and Robotics, Computer Vision and Aeria Robotics (CVAR) Group. H was a Visiting Researcher with the Aerospace Controls Laboratory (ACL), Massachusetts Institute of Technology (MIT), from October to December 2018, for three months. He worked for more than a year in the aerospace sector, contributing to projects from the European Space Agency (ESA). He is currently a Researcher with the Computer Vision and Aerial Robotics (CVAR) Group, Centre for Automation and Robotics, Universidad Politécnica de Madrid. His research interests include deep reinforcement learning techniques applied to aerial robotics, deep learning, aerial robotics, and image processing. He has received several international prizes in UAV competitions, such as IMAV2016, IMAV 2017, and MBZIRC 2020 (Team Leader).

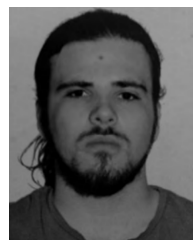

JAVIER RODRIGUEZ-VAZQUEZ received the B.Sc. degree in computer engineering (double major in hardware engineering and computer science) and the M.Sc. degree in systems engineering and computing research from the Universidad de Cádiz (UCA), Cádiz, Spain, in July 2015 and March 2017, respectively. He is currently pursuing the Ph.D. degree in artificial intelligence with the Universidad Politécnica de Madrid (UPM). His research interest includes deep learning methods for solving computer vision tasks, with a special interest in object detection and image segmentation. He has received an international prize in the MBZIRC 2020 Competition. 


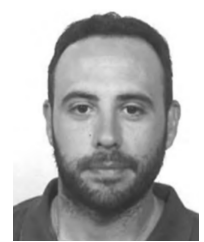

CARLOS SAMPEDRO (Member, IEEE) received the B.Sc. degree in industrial engineering (major in industrial electronics), obtaining the best marks degree award, and the master's degree in automation and robotics from the Universidad Politécnica de Madrid (UPM), Madrid, Spain, in July 2011 and 2014, respectively, and the Ph.D. degree (cum laude) in automation and robotics, in December 2019. He was a Visiting Researcher with Arizona State University, AZ, USA, from September 2015 to December 2015 . He is actively involved in the development of deep reinforcement learning algorithms for autonomous navigation and control of unmanned aerial vehicles (UAVs). His research interest includes the applications of learning-based techniques for solving computer vision problems, with a special interest in object detection and recognition using deep learning techniques. He received the Predoctoral Grant from the Universidad Politécnica de Madrid, in January 2017.

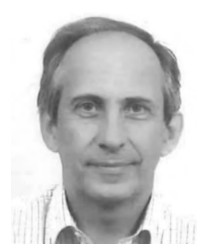

PASCUAL CAMPOY (Senior Member, IEEE) has been a Visiting Professor with Tongii University, Shanghai, China, and QUT, Australia. He is currently a Lecturer in control, machine learning, and computer vision. He is also a Full Professor in automation and robotics with the Universidad Politécnica de Madrid (UPM), Madrid, Spain, and a Visiting Professor with TU Delft, The Netherlands. He is leading the Centre for Automation and Robotics (CAR), Computer Vision and Aerial Robotics (CVAR) Research Group. He has been the Head Director of over 40 research and development projects, including research and development European projects, national research and development projects, and over 25 technological transfer projects directly contracted with the industry. $\mathrm{He}$ is the author of over 200 international scientific publications. He holds nine patents, three of them registered internationally. He has received several international prizes in unmanned aerial vehicle (UAV) competitions, such as IMAV 2012, IMAV 2013, IARC 2014, IMAV2016, IMAV 2017, and MBZIRC 2020. 

Appendices 



\section{Appendix 1}

\section{International Competitions}

Competition results pose an important complementary contribution of the present dissertation. The research and development for the eventual participation in several competitions have led to an enormous knowledge acquired and an outstanding outcome, which has fed other lines of research and industrial projects. In this regard, the present appendix presents a brief explanation of the most relevant results from the competitions which the author of the present doctoral thesis has contributed to. In this dissertation, the competition results are considered to achieve TRL-6, since the whole stack of hardware and software technologies has been tested in a real relevant environment, which is the competition field.

\section{A.1 2020 Mohamed Bin Zayed International Robotics Com- petition (MBZIRC)}

The 2020 Mohamed Bin Zayed International Robotics Competition (MBZIRC) has taken place in Abu Dhabi, United Arab Emirates (UAE), and was composed of four challenges: Challenge 1, 2, 3, and the Grand Challenge. The team, which the author of the present dissertation has been part of as the main lead role, participated in Challenge 1 and got classified for Grand Challenge.

Challenge 1 consisted of an autonomous ball catching from a non-cooperative UAV which was moving at high-speeds. The ball was tethered to the target UAV, which was performing a 2D lemniscate trajectory within a tilted plane. Additionally, a complementary task was proposed where the UAV was required to burst balloons which were tethered to the ground. The complete task was challenging since it involved complex hardware and software development, testing, and validation.

Hence, a complete hardware and software stack was designed and implemented for the challenge. The base UAV platform was DJI Matrice 210 V2 RTK. The UAV was provided with several sensory components, such as GPS, Real-Time Kinematic (RTK), two IMUs, a Zenmuze X3s camera gimbal, two VGA stereo pairs, and one top-looking wide-angular camera. Additionally, a complete gripper was designed and integrated to autonomously catch a tethered object at high-speeds. In Fig. A.1a the complete UAV and its hardware components have been illustrated. Also, in Fig. A.1b, the CAD model of the designed 


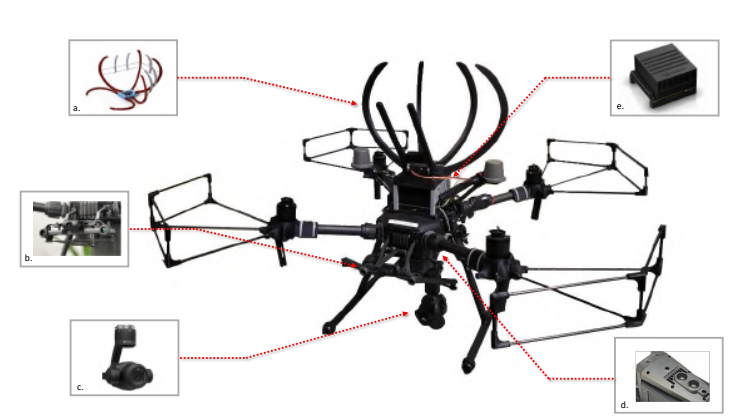

(a)

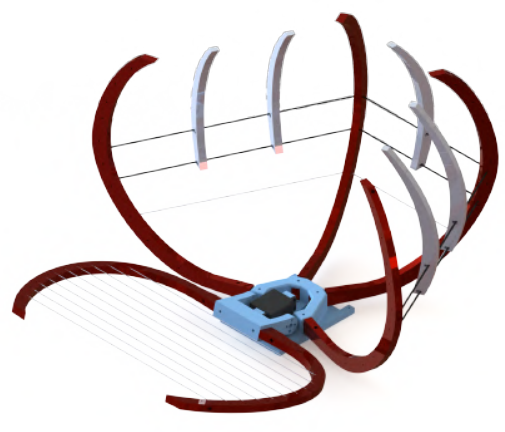

(b)

Figure A.1: (a) Designed UAV platform with hardware components highlighted. (b) Gripper CAD model.

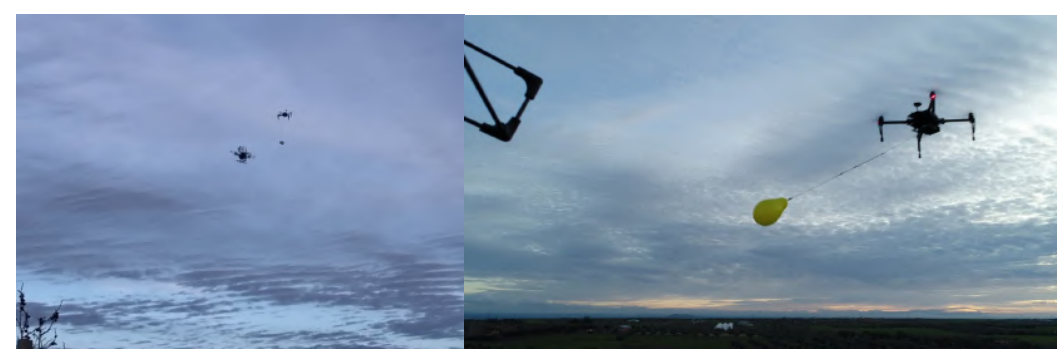

(a)

(b)

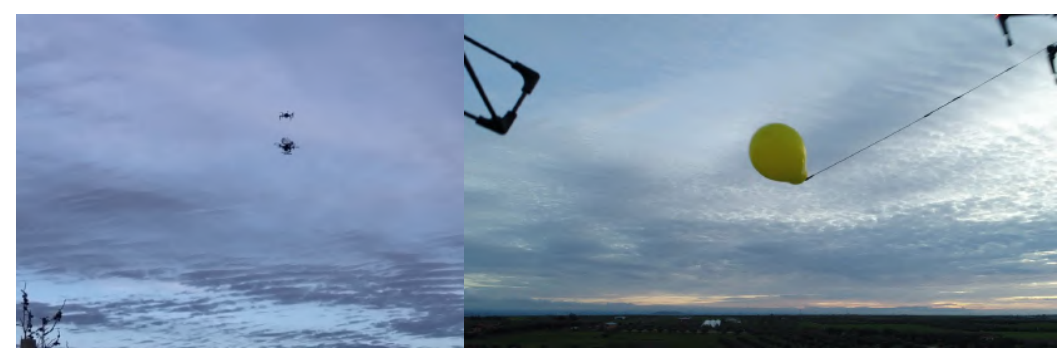

(c)

(d)

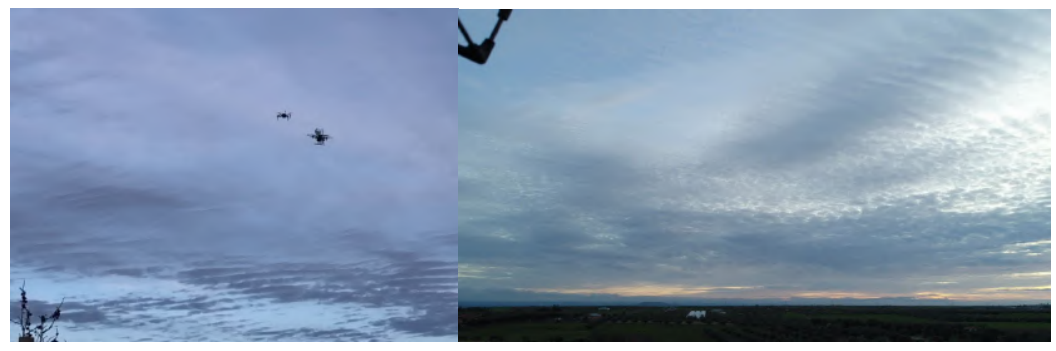

(e)

(f)

Figure A.2: Results from an autonomous grasping maneuver test in an outdoor experimentation environment. (a)(c)(e) Sequence view of the aerial vehicle grasping maneuver. (b) (d) (f) Images from the on-board camera used for detection of the target UAV and ball.

gripper has been depicted. 


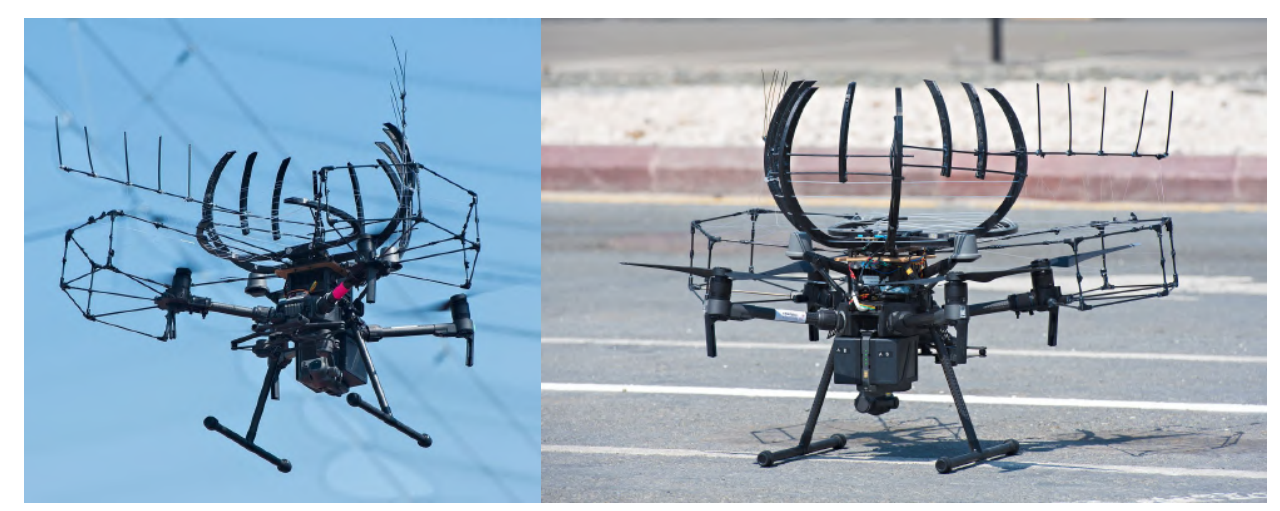

(a)

(b)

Figure A.3: (a) (b) UAV in the real competition environment.

Several simulated and real-flight experiments have been carried out in order to validate the approach in a representative outdoor environment. Among other results, in Fig. A.2, a successful autonomous grasping maneuver has been represented. The UAV was required to perform the full mission which involved search, follow, approach, and grasp maneuvers. The complete hardware and software stack was developed, leading to outstanding results in the real competition environment. In Fig. A.3, the proposed UAV within the real competition environment has been illustrated. The team ended up in $3^{\text {rd }}$ position for the Grand Challenge ${ }^{1}$, which is a parallel combination of the three proposed challenges. A video of the competition has been provided?

\section{A.2 2018 IROS Autonomous Drone Race (IROS ADR) Com- petition}

The 2018 International Conference on Intelligent Robots and Systems (IROS) Autonomous Drone Race (ADR) has taken place in Madrid, Spain, and was composed of one challenge: an autonomous race through different gates. The team, which the author of the present dissertation has been part of as a co-leader role, participated in the stated competition.

The challenge consisted of several gates which the UAVs were required to cross in the shortest possible time. For this particular competition, the available time and human resources were limited. In this context, a simple approach with a consumer multirotor (Parrot Bebop 2) was designed. Indeed, the whole approach was computed on-board with an Intel Up-Board and the algorithms developed were completely vision-based (see Fig. A.4.

Thus, numerous amount of experiments were performed in both simulated and realflight environments. In Fig. A.5a a simulated replica of the competitive environment has been generated. In Fig. A.5b a real replica of the environment was built in a controlled laboratory area. The thorough experimentation provided successful results in the laboratory environment. However, the performance in the competition was poor, ending up in $7^{\text {th }}$ position ${ }^{3}$.

\footnotetext{
${ }_{1}^{1}$ https://www.mbzirc.com/winning-teams/2020/challenge4

2 https://vimeo.com/399137661

3 https://www.iros2018.org/competitions
} 


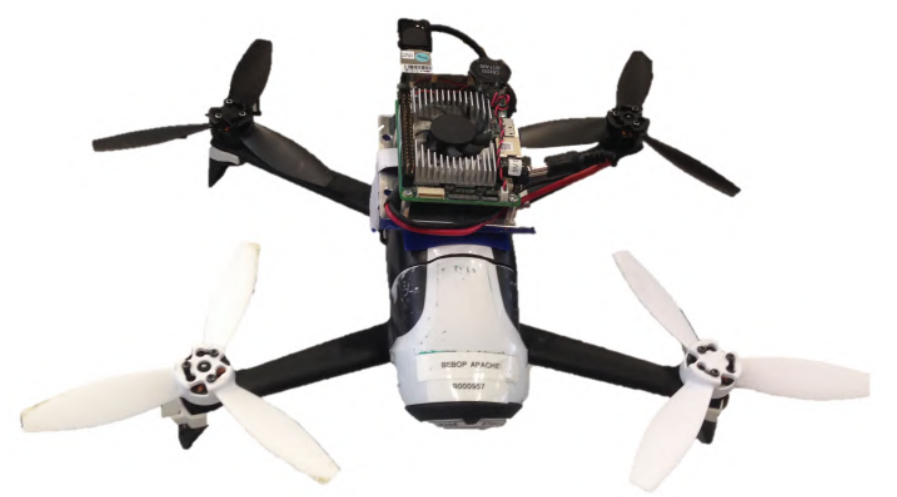

Figure A.4: Utilized UAV platform (Parrot Bebop 2) with hardware components (Intel UpBoard).

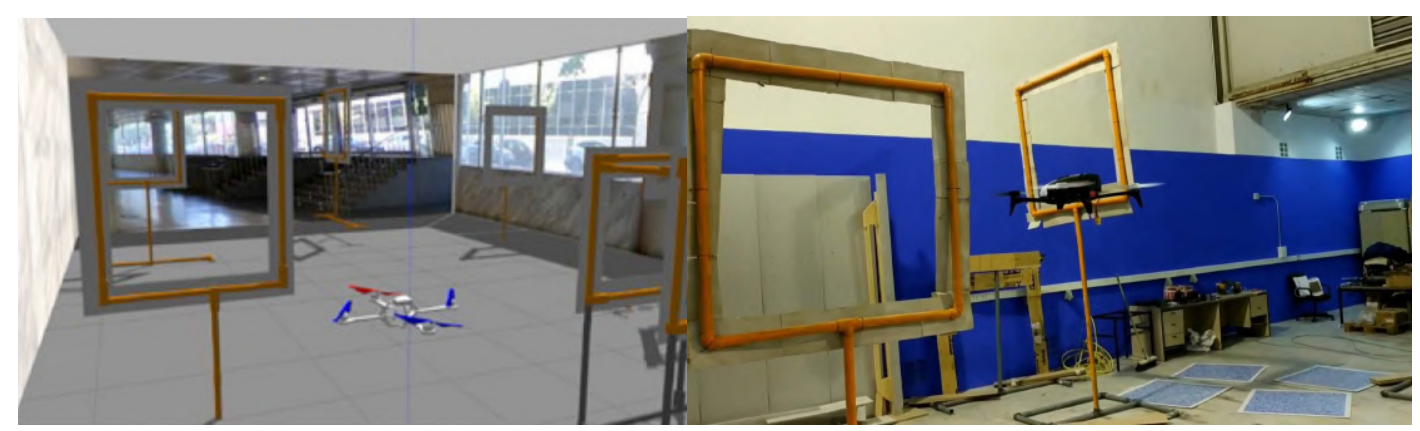

(a)

(b)

Figure A.5: Simulated and real replica of the IROS ADR 2018 environment (a) Simulated environment. (b) Real environment.

\section{A.3 2017 International Micro Aerial Vehicles (IMAV) Com- petition}

The 2017 IMAV competition has taken place in Toulouse, France, and was also composed of two challenges: an indoor and an outdoor challenge. Again, the team, which the author of the present dissertation has been part of as a co-leader role, participated in the indoor challenge.

The indoor challenge consisted of an unstructured indoor environment with several tasks to be autonomously carried out. The tasks involved autonomous window/pipe crossing, column-cluttered environment crossing, vegetation area navigation, wind gusts area crossing, and landing on a moving platform, which was solely moving in orientation (3DOF). In this case, the competition was approached in a simpler yet effective trend, with a consumer UAV platform (Parrot Bebop 2) and an off-the-shelf SLAM-based stereo camera pair (Parrot Slam Dunk). The stated hardware configuration has been illustrated in Fig. A.6.

A swarm of UAVs was designed to perform several tasks of the challenge in parallel, in order to reduce performance times and maximize the points achieved. Extensive experimentation was carried out in a replica of the competition environment, built in a controlled laboratory area. In Fig. A.7 an autonomous window crossing of one of the deployed UAVs is depicted. The extensive testing carried out allowed for successful performance in the 


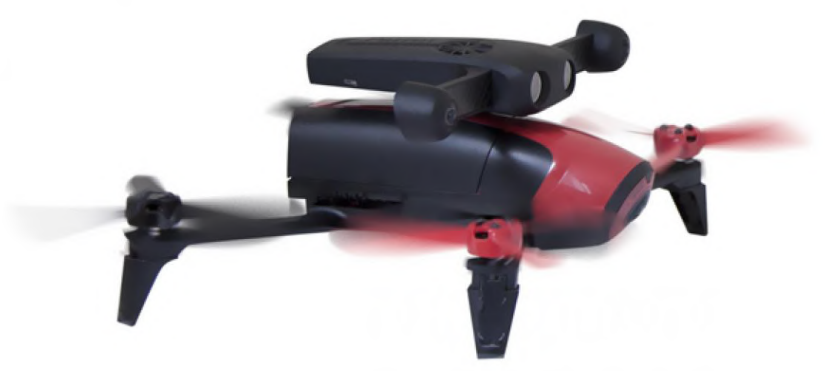

Figure A.6: Utilized UAV platform (Parrot Bebop 2) with hardware components (Parrot Slam Dunk).

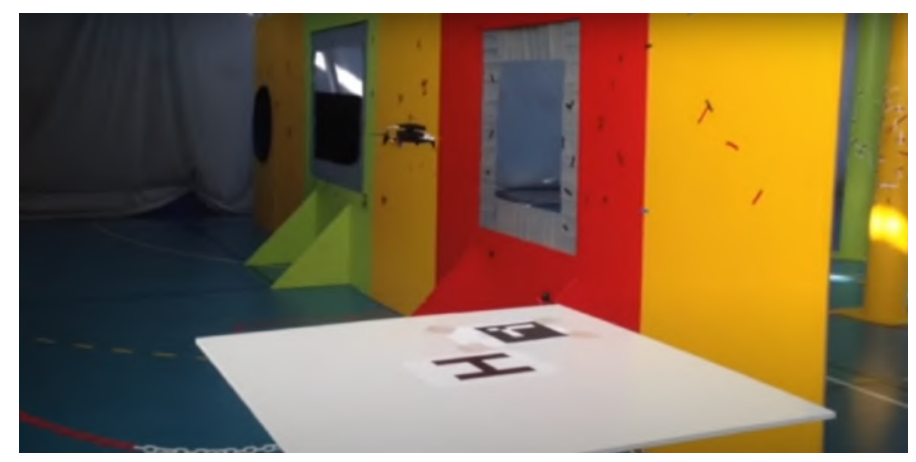

Figure A.7: Real competition environment for the IMAV 2017 indoor competition.

real competition environment, achieving the $2^{\text {nd }}$ position for the indoor competition ${ }^{4} \mathrm{~A}$ summary video has been provided 5 .

\section{A.4 2016 International Micro Aerial Vehicles (IMAV) Com- petition}

The 2016 International Micro Aerial Vehicles (IMAV) has taken place in Beijing, China, and was composed of two challenges: an indoor and an outdoor challenge. The team, which the author of the present dissertation has been part of, participated in the indoor challenge.

The indoor challenge consisted of an unstructured indoor environment where the UAV was required to autonomously navigate in order to pick and release certain objects which were randomly located inside. The UAV could optionally enter, in order of complexity, through the door, the window, or the chimney. Additionally, the UAV was proposed to autonomous land on a moving platform which was located in the surroundings of the indoor environment. A fully custom UAV was designed for the challenge (see Fig. A.8) and onboard sensors were integrated. The on-board computer consisted of an Intel NUC6i5SYK featuring a $2.9 \mathrm{GHz}$ Intel Core i5-6260U CPU. The avionics of the UAV was managed by a Pixhawk Meier et al. (2011) autopilot, which integrated an IMU, a barometer, and

${ }^{4}$ http: //www . imavs .org/2017/index .html@p=410.html

https://ww . youtube .com/watch?v=HOR8kDi8EDI 


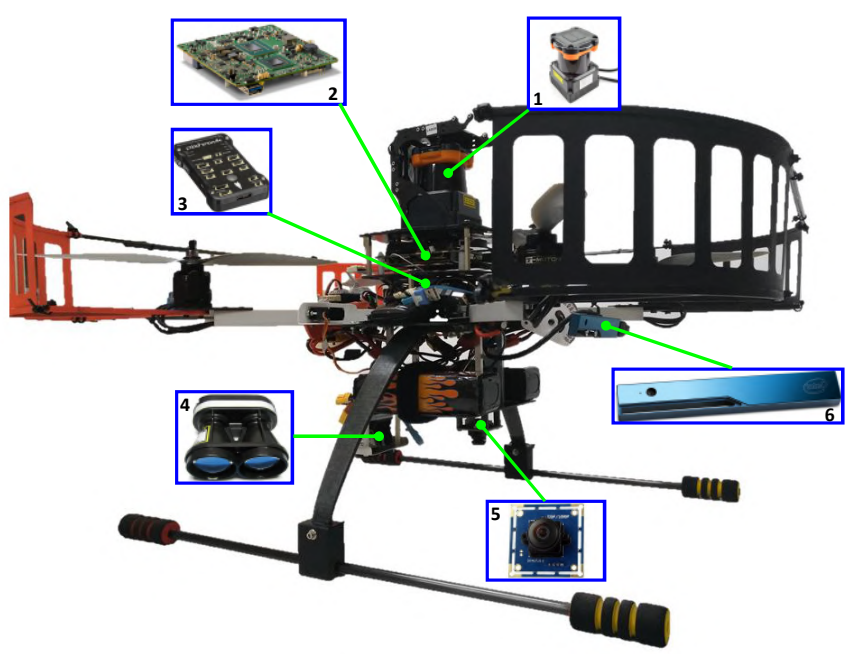

Figure A.8: Designed UAV platform with hardware components highlighted.

a magnetometer. The exteroceptive sensors mounted on-board consisted of a Hokuyo laser rangefinder UTM-30LX with a horizontal field of view of $270^{\circ}$, an Intel Realsense R200 camera, a standard RGB $180^{\circ}$ fisheye-lens bottom-looking camera, and a Lightware SF10/A altimeter with a maximum range of $25 \mathrm{~m}$. Stated sensory and computational resources allowed for performing the mission in autonomous mode with all the processing carried out on-board.

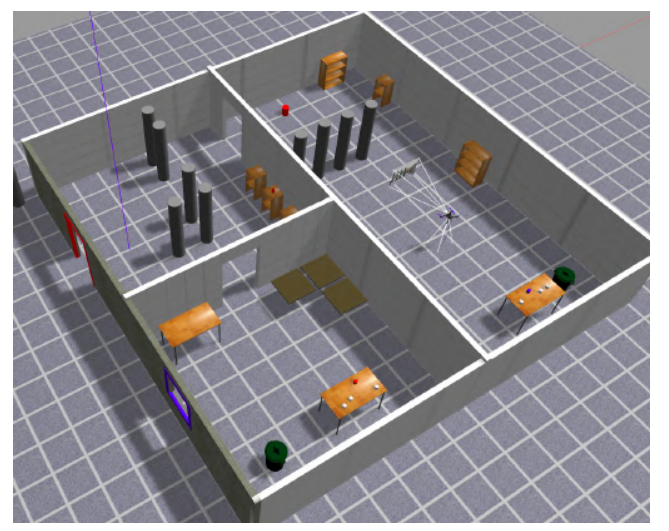

Figure A.9: Simulated replica of the IMAV 2016 indoor competition environment.

A simulated replica of the IMAV 2016 indoor competition environment was generated in Gazebo in order to perform several experiments in a Software-In-The-Loop (SITL) simulation (see Fig. A.9p. The simulation allowed for the early debugging of the algorithms and the continuous development of new components or the improvement of the existing ones.

Additionally, a real partial replica of the IMAV 2016 indoor environment was built to perform thorough experimentation in relevant conditions. An unstructured and cluttered indoor environment and a moving platform, among others, were generated to the stated aim (see Fig. A.10). A notable amount of experiments were carried out for validating the approach, such as autonomous entering/exiting to/from the indoor environment, navigation in cluttered areas, the release of items in predefined buckets, and landing on top of a moving platform. The landing technique utilized for landing on top of a moving platform 


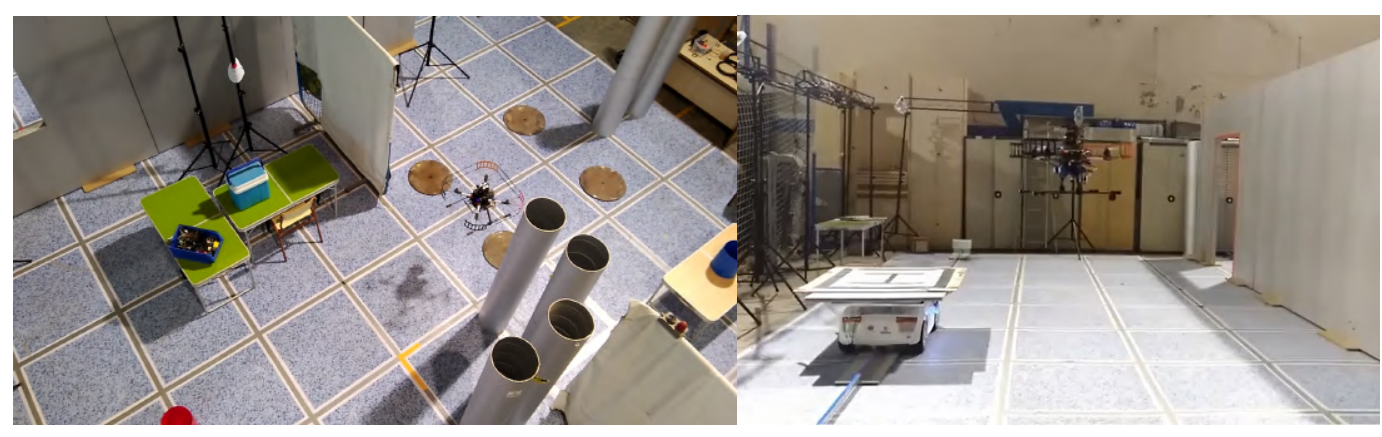

(a)

(b)

Figure A.10: Real partial replica of the IMAV 2016 indoor environment (a) The UAV is autonomously navigating through an unstructured and cluttered indoor environment. (b) The UAV is autonomously landing on a moving platform, utilizing technique provided in Section 4.3 .2 .

corresponds to the method detailed in Section 4.3.2. The extensive testing carried out in both simulated and real environments allowed for successful performance in the real competition environment, achieving the $4^{\text {th }}$ position for the indoor competition 6 Three summary videos have been provided服.

\footnotetext{
6 http://www .imavs.org/2016/documents/Result.pdf

'https ://www . youtube.com/watch?v=qon8UK0f vwk

${ }^{\varepsilon}$ https://www. youtube.com/watch?v=Xhw03w2bxFA

https : //www . youtube.com/watch?v=9_06Gt04-70
} 



\section{3}

\section{Industrial Project}

Although the primary aim of this thesis was to develop novel machine learning techniques for aerial robotics, other innovative methods that do not include learning strategies have also been able to solve real-world industrial problems. In this scenario, the author of this dissertation has contributed to several adjacent research projects, such as windmill inspection or fuel tank inspection. Also, a relevant project which the author of this thesis, together with Bavle (2019), has contributed to, is the autonomous UAV inspection of a power plant boiler. On this basis, and in order to provide additional TRL-6 relevant results, a brief explanation of this successful project for autonomous UAV power plant inspection has been included in this appendix.

\section{B.1 Autonomous UAV Power Plant Inspection}

Power plants, and in particular, coal-based power plants include a boiler, where the coal is burned to initiate the energy transformation process. The aforementioned boilers suffer from erosion, deterioration, fractures, etc. and periodic inspection and maintenance are normally required. The usual method for inspection is human-based, with climbers who can visually inspect key zones from the interior of the boiler. In this context, the development of autonomous UAVs for the inspection of a boiler from a power plant provides several benefits in terms of time, economic, and safety reasons. For the case of this particular project, the UAV (see Fig. B.1) was meant to validate successful autonomous navigation inside a boiler from a power plant. The dimensions of the boiler were approximately 18 $\mathrm{m} \times 18 \mathrm{~m} \times 60 \mathrm{~m}$. The project was considered completed when the UAV was able to perform autonomous navigation within the boiler volume and to record a video of a special element, the burners.

The UAV (DJI Matrice 100) was equipped with a DJI Manifold on-board computer, a Hokuyo UTM-30LX 2D-LIDAR, a Lightware SF/30C 1D-LIDAR altimeter, on-board illumination and a high-resolution Zenmuse Z3 RGB camera gimbal (see Fig. B.2). Covering protection was also included in order to properly perform the entrance maneuver to the boiler, which had to be carried out from the bottom of the volume and involved risks (see 


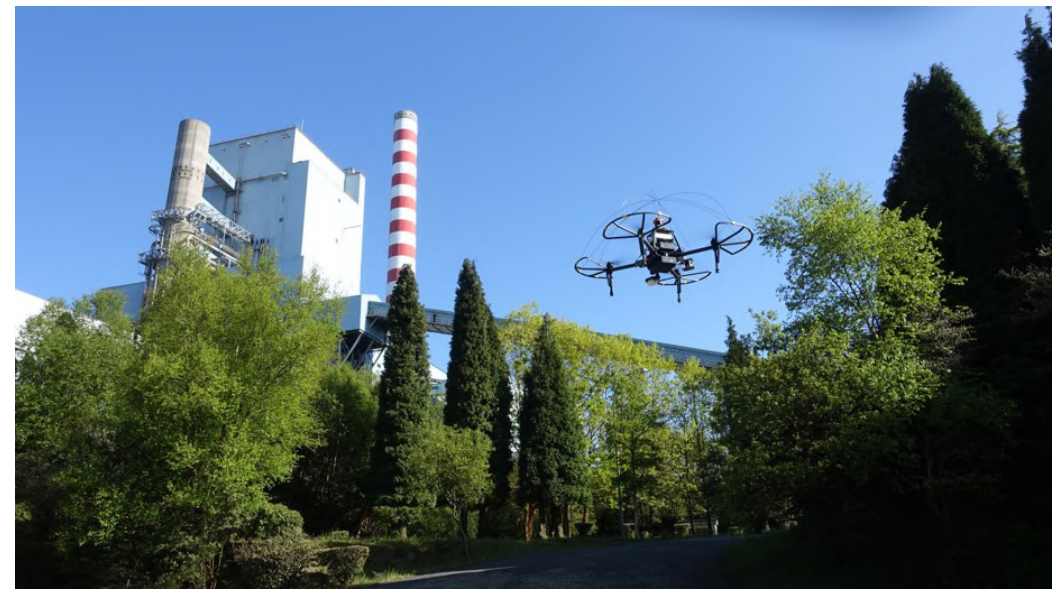

Figure B.1: Representative image of the developed UAV platform (DJI Matrice 100) in the outdoor surroundings of the power plant.

Fig. B.1 and B.3b. The software architecture was based on Aerostack 1 , which is an opensource multi-purpose software framework for the development of autonomous multi-robot unmanned aerial systems created by the Computer Vision and Aerial Robotics (CVAR) group. The software development for this particular project involved the implementation or integration of an altitude estimator, a 2D-SLAM algorithm, a path planning algorithm, a navigation interface, a mission planner, and a motion controller, among others. A summary video of the project has been provided ${ }^{2}$

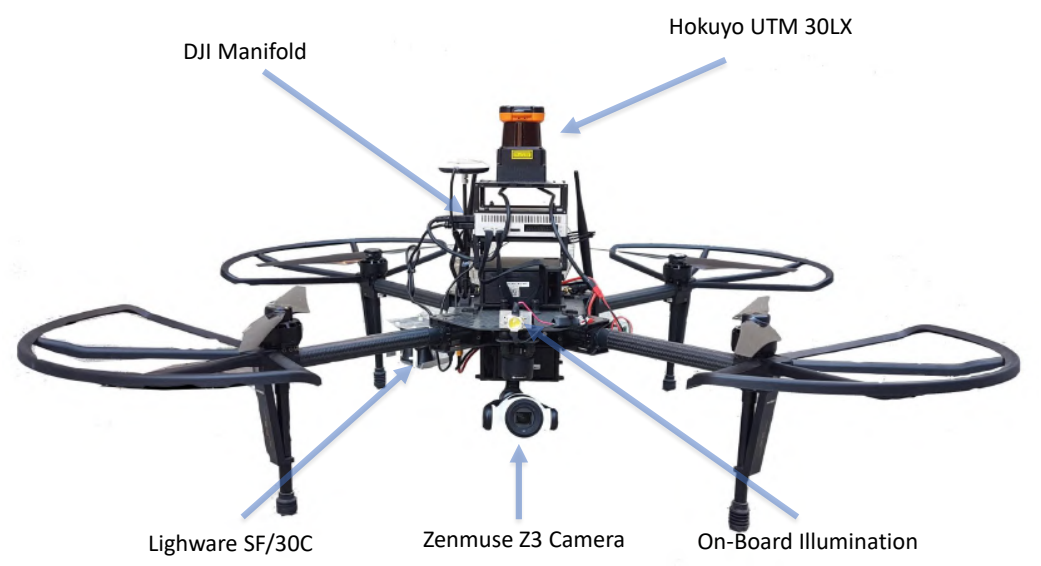

Figure B.2: Implemented UAV platform with hardware components.

As an initial approximation to the project, a simulated environment was generated in Gazebo simulator (see Fig. B.3a) in order to solve early issues and to gather information about the complete challenge. All the modules were debugged at this stage and allowed to perform real laboratory tests. In a latter stage, the UAV was validated in the Meirama Power Plant, which is a relevant industrial environment (see Fig. B.3b. An autonomous UAV navigation was carried out and the burners were recorded.

The project ended up successfully with autonomous UAV navigation inside the boiler

1 www.aerostack.org

2 https ://vimeo.com/269340493 


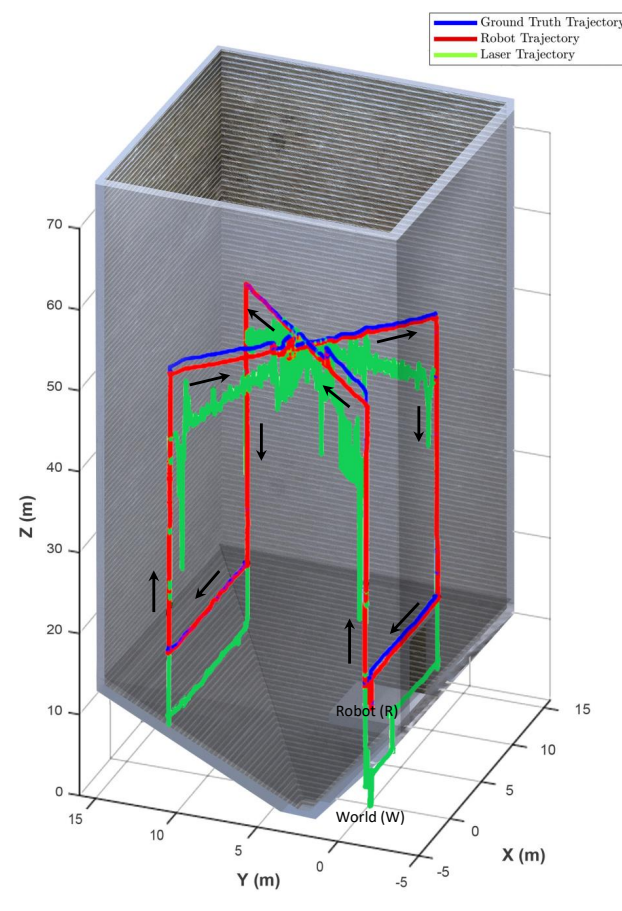

(a)

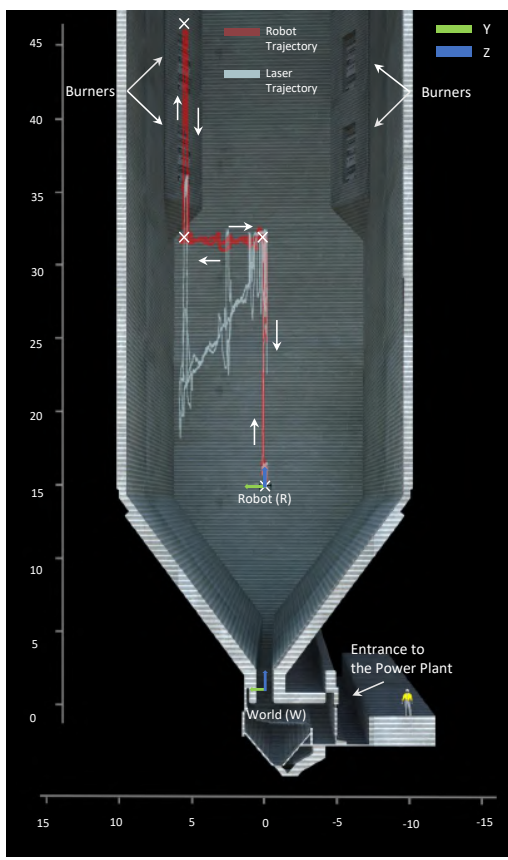

(b)

Figure B.3: Simulated and real-flight experiments of the autonomous UAV navigation in a power plant boiler. (a) Simulated trajectory of the UAV (ground-truth and estimated) and 1D-LIDAR altimeter measurements. (b) Real-flight trajectory of the UAV (estimated) and 1D-LIDAR altimeter measurements.

limits in order to record the burners located in one of the boiler corners. The UAV was able to perform the maneuver in the stated hazardous environment (limited light, suspended dust, uneven surfaces, etc.). Although the complete experiment was adequately carried out, a higher level of autonomy is required for in-production boiler inspection. The entrance and exit to/from the boiler were performed in manual mode and the potential obstacles within the volume were not considered at this stage. As an immediate future work, a structured-light visual odometry algorithm can be implemented in order to be able to infer localization even in the absence of light, in the surrounding of highly uneven surfaces, and with obstacles included in the volume. 



\section{Appendix}

\section{Scientific Dissemination}

\section{C.1 Journal Publications}

2020

Molina, M., Carrera, A., Camporredondo, A., Bavle, H., Rodriguez-Ramos, A., and Campoy, P. (2020). Building the executive system of autonomous aerial robots using the Aerostack open-source framework. International Journal of Advanced Robotic Systems, 17(3), 1729881420925000. JCR (2018): 1.223

Rodriguez-Ramos, A., Rodriguez-Vazquez, J., Sampedro, C., and Campoy, P. (2020). Adaptive Inattentional Framework for Video Object Detection With Reward-Conditional Training. IEEE Access, 8, 124451-124466. JCR (2019): 4.670

2019

Molina, M., Camporredondo, A., Bavle, H., Rodriguez-Ramos, A., and Campoy, P. (2019). An execution control method for the Aerostack aerial robotics framework. Frontiers of Information Technology \& Electronic Engineering, 20(1), 60-75.

Rodriguez-Ramos, A., Alvarez-Fernandez, A., Bavle, H., Campoy, P., and How, J. P. (2019). Vision-based multirotor following using synthetic learning techniques. Sensors, 19(21), 4794. JCR (2019): 3.275

Sampedro, C., Rodriguez-Ramos, A., Bavle, H., Carrio, A., de la Puente, P., and Campoy, P. (2019). A fully-autonomous aerial robot for search and rescue applications in indoor environments using learning-based techniques. Journal of Intelligent \& Robotic Systems, 95(2), 601-627. JCR (2018): 2.020

Sampedro, C., Rodriguez-Vazquez, J., Rodriguez-Ramos, A., Carrio, A., and Campoy, P. (2019). Deep learning-based system for automatic recognition and diagnosis of electrical insulator strings. IEEE Access, 7, 101283-101308. JCR (2018): 4.098 
Rodriguez-Ramos, A., Sampedro, C., Bavle, H., De La Puente, P., and Campoy, P. (2018). A deep reinforcement learning strategy for UAV autonomous landing on a moving platform. Journal of Intelligent \& Robotic Systems, 93(1-2), 351-366. JCR (2018): 2.020

Bavle, H., Sanchez-Lopez, J. L., De la Puente, P., Rodriguez-Ramos, A., Sampedro, C., and Campoy, P. (2018). Fast and robust flight altitude estimation of multirotor UAVs in dynamic unstructured environments using 3D point cloud sensors. Aerospace, 5(3), 94. CiteScore (SCOPUS): 1.58

\section{7}

Carrio, A., Sampedro, C., Rodriguez-Ramos, A., and Campoy, P. (2017). A review of deep learning methods and applications for unmanned aerial vehicles. Journal of Sensors, 2017. JCR (2017): 2.057

\section{C.2 Conference Publications}

\section{8}

Sampedro, C., Rodriguez-Ramos, A., Gil, I., Mejias, L., and Campoy, P. (2018, October). Image-based visual servoing controller for multirotor aerial robots using deep reinforcement learning. In 2018 IEEE/RSJ International Conference on Intelligent Robots and Systems (IROS) (pp. 979-986). IEEE.

Rodriguez-Ramos, A., Sampedro, C., Bavle, H., Moreno, I. G., and Campoy, P. (2018, October). A Deep Reinforcement Learning Technique for Vision-Based Autonomous Multirotor Landing on a Moving Platform. In 2018 IEEE/RSJ International Conference on Intelligent Robots and Systems (IROS) (pp. 1010-1017). IEEE.

Sampedro, C., Bavle, H., Rodriguez-Ramos, A., de la Puente, P., and Campoy, P. (2018, October). Laser-based reactive navigation for multirotor aerial robots using deep reinforcement learning. In 2018 IEEE/RSJ International Conference on Intelligent Robots and Systems (IROS) (pp. 1024-1031). IEEE.

Bavle, H., Manthe, S., de la Puente, P., Rodriguez-Ramos, A., Sampedro, C., and Campoy, P. (2018, October). Stereo visual odometry and semantics based localization of aerial robots in indoor environments. In 2018 IEEE/RSJ International Conference on Intelligent Robots and Systems (IROS) (pp. 1018-1023). IEEE.

\section{7}

Sampedro, C., Bavle, H., Rodríguez-Ramos, A., Carrio, A., Fernández, R. A. S., Sanchez-Lopez, J. L., and Campoy, P. (2017, June). A fully-autonomous aerial robotic solution for the 2016 international micro air vehicle competition. In 2017 International conference on unmanned aircraft systems (ICUAS) (pp. 989-998). IEEE.

Bavle, H., Sanchez-Lopez, J. L., Rodriguez-Ramos, A., Sampedro, C., and Campoy, P. (2017, June). A flight altitude estimator for multirotor UAVs in dynamic and unstructured indoor environments. In 2017 International Conference on Unmanned Aircraft Systems 
(ICUAS) (pp. 1044-1051). IEEE.

Rodriguez-Ramos, A., Sampedro, C., Bavle, H., Milosevic, Z., Garcia-Vaquero, A., and Campoy, P. (2017, June). Towards fully autonomous landing on moving platforms for rotary unmanned aerial vehicles. In 2017 International Conference on Unmanned Aircraft Systems (ICUAS) (pp. 170-178). IEEE.

\section{6}

Sampedro, C., Bavle, H., Sanchez-Lopez, J. L., Fernández, R. A. S., Rodríguez-Ramos, A., Molina, M., and Campoy, P. (2016, June). A flexible and dynamic mission planning architecture for uav swarm coordination. In 2016 International Conference on Unmanned Aircraft Systems (ICUAS) (pp. 355-363). IEEE.

Rodríguez-Ramos, A., Sampedro, C., Carrio, A., Bavle, H., Fernández, R. A., Milo?evi?, Z., and Campoy, P. (2016, October). A monocular pose estimation strategy for uav autonomous navigation in gnss-denied environments. In Proceedings of the International Micro Air Vehicle Conference and Flight Competition, Beijing, China (pp. 17-22). 


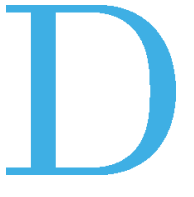

\section{Unpublished Results}

Parallel unpublished studies have been carried out towards one of the main objectives of this dissertation, which is the exploration of techniques that make use of synthetic information to facilitate the development of learning-based algorithms within the robotics context. In this line, photorealistic simulators offer the possibility of synthetic images generation and labeling on a wide scale. Nevertheless, although the generated images can be realistic, they could not achieve the required level of complexity of reality, in terms of illumination, object types, textures, defects, etc. In this appendix, a successful strategy to overcome the stated challenges due to differences between the synthetic and real domains is explored for the particular application of collision detection. Furthermore, preliminary results are provided to validate the proposed approach.

\section{D.1 Domain Adaptation for Collision Detection}

Commonly, in order to solve an application case with standard supervised learning methods, a representative amount of annotated data is required. However, annotated data can be economically costly or, in some scenarios, it can be completely unavailable. For a case where the application involves the usage of images, photorealistic simulators or game engines can be utilized to speed up the dataset generation or to augment the already-available real dataset. However, the synthetic domain is normally far from the real domain, and hence, the images (although realistic) cannot be directly used for a successful training pipeline. In this context, several techniques have been explored in the literature (see Section 2 and P.II). In this appendix, to fulfill the virtual-to-reality gap and to make use of pure synthetic images for training, a Cycle GAN (cGAN) architecture Zhu et al. (2017) has been proposed to adapt the synthetic domain to the real one, and vice versa.

The cGAN architecture is focused on the case of two unpaired image domains, where there exist examples of images in both domains but in completely arbitrary scenarios (see Fig. D.1). For the case under study, where the main goal is to generate the probability of collision in a structured environment (such as roads, corridors, etc.) based solely on images, two types of images are required in every domain: one corresponding to a safe situation and another one corresponding to a likely collision. The real image domain $X$ corresponds 
to a public dataset ${ }^{1}$ and the synthetic image domain $Y$ corresponds to a custom dataset generated under the Unreal2 game engine.

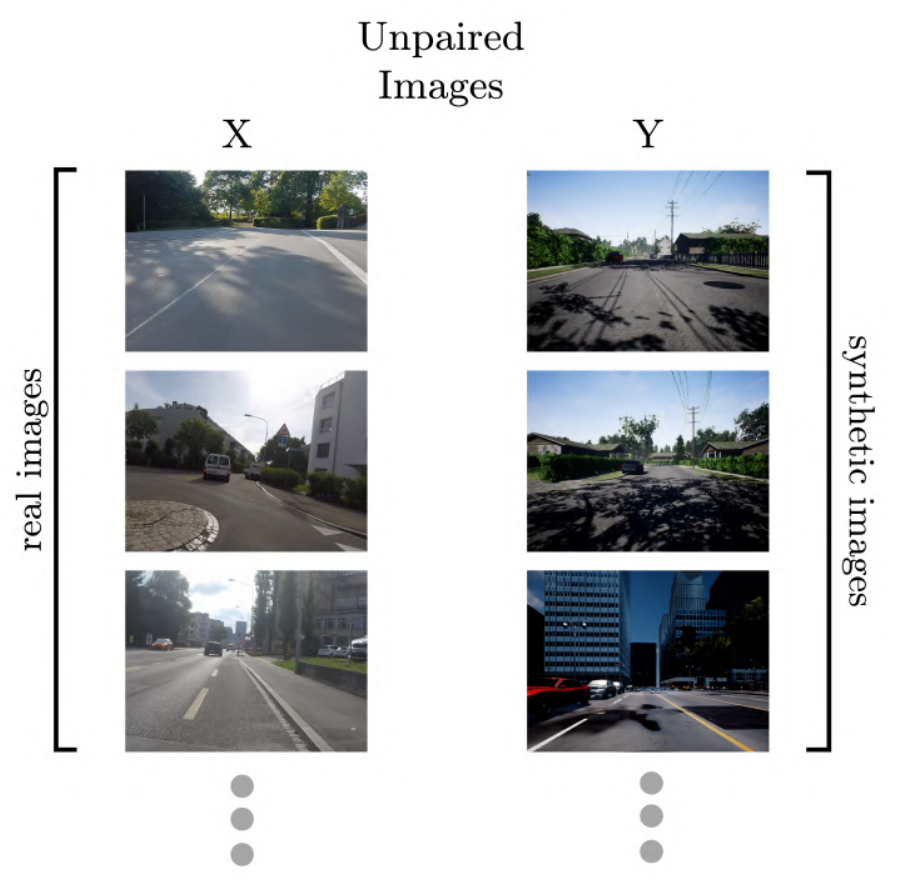

Figure D.1: Unpaired training images from two domains. $X$ corresponds to the realdomain images extracted from a public dataset. $Y$ corresponds to the synthetic-domain images generated under Unreal game engine.

The cGAN framework involves training two mapping functions (generators) $G$ and $F$, and two discriminators $D_{X}$ and $D_{Y}$ (see Fig. D.2). $D_{Y}$ encourages $G$ to translate $X$ into outputs indistinguishable from domain $Y$, and vice versa for $D_{X}$ and $F$. To further regularize the mappings, two cycle-consistency losses were introduced, which capture the intuition that whether one domain is translated to the other and back again it should arrive at where it started. A forward cycle-consistency loss $x \rightarrow G(x) \rightarrow F(G(x)) \approx x$, and backward cycle-consistency loss $y \rightarrow F(y) \rightarrow G(F(y)) \approx y$ were introduced for training purposes.

All of the mapping functions and discriminator were trained as in Zhu et al. (2017) and $F$ generator was isolated to adapt the complete synthetic dataset $X$ to the real domain (see Fig. D.3), leading to an adapted dataset $\hat{X}$. As explained, in order to validate the domain adaptation technique in a real robotic application, a collision detector has been proposed. The selected collision detector architecture has been the Dronet model architecture, as in Loquercio et al. (2018), due to its versatility and reduced number of parameters. Additionally, the selection of Dronet enabled the direct comparison of our proposed approach to their original model, which was trained with the real dataset.

The experimentation consisted of training Dronet with three different datasets: real $X$, synthetic $Y$ and domain-adapted $\hat{X}$ datasets. The evaluation of the models has been carried out with the same testing dataset of the real domain. The accuracy metric has been chosen for evaluation in this preliminary stage of experimentation. This metric is sufficient to evaluate the benefits of the approach. In Table D.1, the accuracy metric is shown for

\footnotetext{
${ }_{1}^{1}$ http://rpg.ifi.uzh.ch/data/collision.zip

2 https://ww .unrealengine.com/en-US/
} 


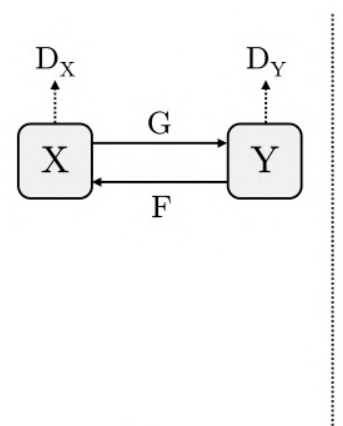

(a)

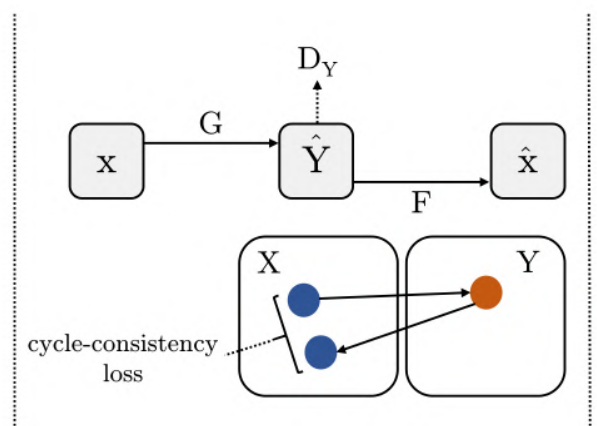

(b)

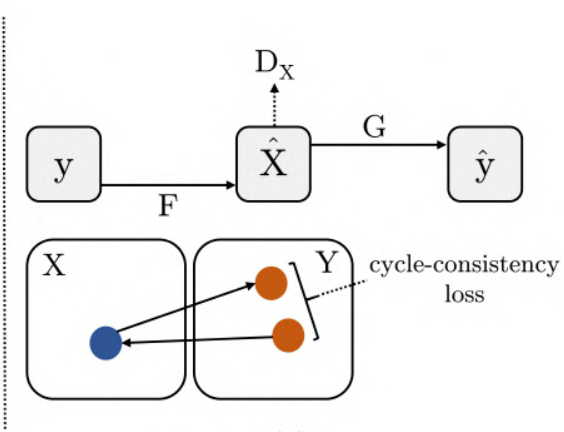

(c)

Figure D.2: Domain adaptation model architecture. The model contains two mapping functions $G: X \rightarrow Y$ and $F: Y \rightarrow X$, and associated discriminators $D_{Y}$ and $D_{X}$. $D_{Y}$ encourages $G$ to translate $X$ into outputs indistinguishable from domain $Y$, and vice versa for $D_{X}$ and $F$. To further regularize the mappings, two cycle consistency losses were introduced, which capture the intuition that whether one domain is translated to the other and back again it should arrive at where it started: (b) forward cycle-consistency loss: $x \rightarrow$ $G(x) \rightarrow F(G(x)) \approx x$, and (c) backward cycle-consistency loss: $y \rightarrow F(y) \rightarrow G(F(y)) \approx y$.

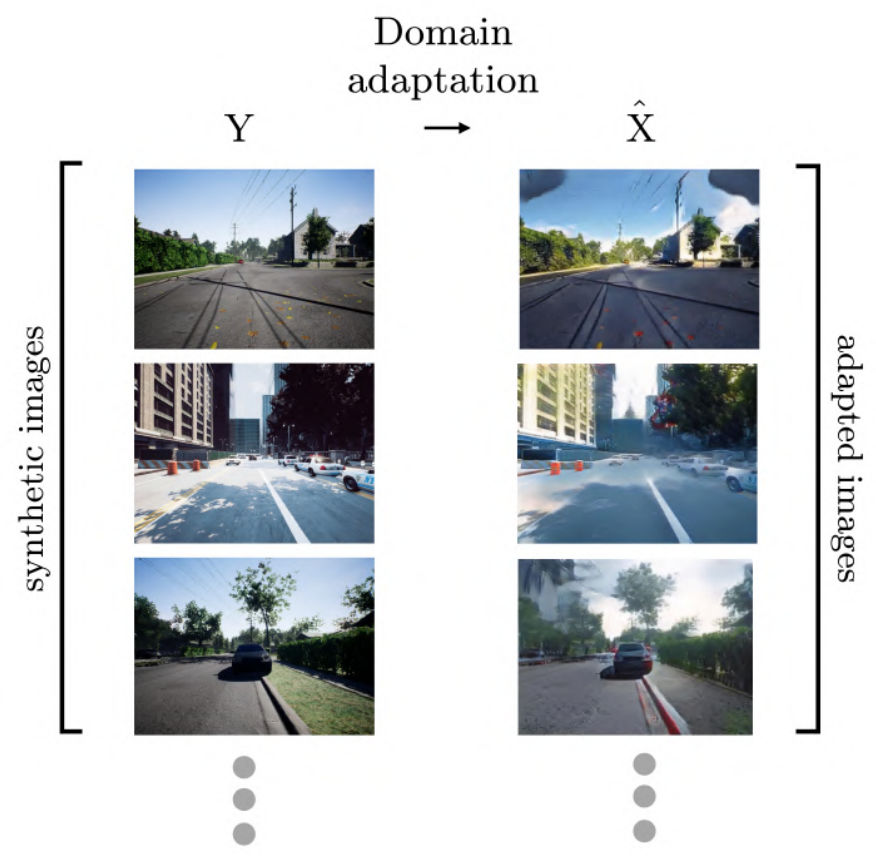

Figure D.3: Examples of domain-adapted images. The synthetic images have been translated into the real domain in order to train a model for collision detection.

the three dataset scenarios. As shown, the synthetic dataset provided a poor performance of 0.62 in accuracy, whereas the domain-adapted dataset yielded a performance of 0.94 in accuracy. In comparison to the real dataset domain which provided a 0.954 in accuracy, the domain-adapted dataset has shown an equivalent performance. A video of several real scenarios of both safe and collision environments (running the network which was trained with the domain-adapted dataset) has been provided ${ }^{3}$. Finally, it has to be noted that the

\footnotetext{
$\sqrt[3]{\text { https : //vimeo.com/454798507/08e3c57ed0 }}$
} 
Table D.1: Accuracy results on the test dataset. The real dataset corresponds to a public dataset. The synthetic and domain-adapted dataset were generated based on Unreal game engine.

\begin{tabular}{c|c}
\hline Accuracy (real) & 0.954 \\
\hline Accuracy (synthetic) & 0.62 \\
\hline Accuracy (domain-adapted) & 0.94 \\
\hline
\end{tabular}

synthetic dataset has been automatically labeled and involved a few days of development, whereas the real dataset has involved real sensors mounted on a vehicle (car and bicycle), with large predefined routes which were required to be followed by the stated vehicle in order to store a representative amount of images for the dataset. This fact highlights one of the main benefits of this technique, where a vision-based application can be solved with pure synthetic data, providing state-of-the-art performance, at scale, and reducing the employed resources. Nevertheless, these techniques require the formal evaluation of possible scenarios where the real domain is farther than in the presented application, or the case of more complex applications. 



\section{Bibliography}

Aker, C. and Kalkan, S. Using deep networks for drone detection. In 2017 14th IEEE International Conference on Advanced Video and Signal Based Surveillance (AVSS), pages 1-6. IEEE, 2017.

Akhloufi, M. A., Arola, S. and Bonnet, A. Drones chasing drones: Reinforcement learning and deep search area proposal. Drones, vol. 3(3), page 58, 2019.

Albuquerque, P. L. F. Domain Adaptation in Unmanned Aerial Vehicles Landing Using Reinforcement Learning. PhD Thesis, University of Nebraska-Lincoln, 2019.

Ananthakrishnan, U., Akshay, N., Manikutty, G. and Bhavani, R. R. Control of quadrotors using neural networks for precise landing maneuvers. In Artificial Intelligence and Evolutionary Computations in Engineering Systems, pages 103-113. Springer, 2017.

Anderson, K. and Gaston, K. J. Lightweight unmanned aerial vehicles will revolutionize spatial ecology. Frontiers in Ecology and the Environment, vol. 11(3), pages 138-146, 2013.

Andrychowicz, O. M., Baker, B., Chociej, M., Jozefowicz, R., McGrew, B., Pachocki, J., Petron, A., Plappert, M., Powell, G., Ray, A. et al. Learning dexterous in-hand manipulation. The International Journal of Robotics Research, vol. $39(1)$, pages 3-20, 2020.

Araar, O., Aouf, N. and Vitanov, I. Vision based autonomous landing of multirotor uav on moving platform. Journal of Intelligent \& Robotic Systems, vol. 85(2), pages 369-384, 2017.

Ba, J., Mnin, V. and Kavukcuoglu, K. Multiple object recognition with visual attention. arXiv preprint arXiv:1412.7755, 2014.

Baca, T., Stepan, P. and Saska, M. Autonomous landing on a moving car with unmanned aerial vehicle. In 2017 European Conference on Mobile Robots (ECMR), pages 1-6. IEEE, 2017.

Bahill, A. T. and Gissing, B. Re-evaluating systems engineering concepts using systems thinking. IEEE Transactions on Systems, Man, and Cybernetics, Part C (Applications and Reviews), vol. 28(4), pages 516-527, 1998.

Bähnemann, R., Pantic, M., Popović, M., Schindler, D., Tranzatto, M., Kamel, M., Grimm, M., Widauer, J., Siegwart, R. and Nieto, J. The ethmav team in the mbz international robotics challenge. Journal of Field Robotics, vol. 36(1), pages 78-103, 2019. 
Bansal, S., Akametalu, A. K., Jiang, F. J., Laine, F. and Tomlin, C. J. Learning quadrotor dynamics using neural network for flight control. In 2016 IEEE 55th Conference on Decision and Control (CDC), pages 4653-4660. IEEE, 2016.

BAVle, H. Positioning and mapping for aerial robots using on-board perception for autonomous missions, 2019.

Bellver, M., Giró-i Nieto, X., Marqués, F. and Torres, J. Hierarchical object detection with deep reinforcement learning. arXiv preprint arXiv:1611.03718, 2016.

Beul, M., Houben, S., Nieuwenhuisen, M. and Behnke, S. Fast autonomous landing on a moving target at mbzirc. In 2017 European Conference on Mobile Robots (ECMR), pages 1-6. IEEE, 2017.

Beul, M., Nieuwenhuisen, M., Quenzel, J., Rosu, R. A., Horn, J., Pavlichenko, D., Houben, S. and Behnke, S. Team nimbro at mbzirc 2017: Fast landing on a moving target and treasure hunting with a team of micro aerial vehicles. Journal of Field Robotics, vol. 36(1), pages 204-229, 2019.

Bischoff, R. From research to products: The development of the kuka light-weight robot. In 40th International Symposium on Robotics, Barcelona, Spain. 2009.

BochkovskiY, A., Wang, C.-Y. and LiaO, H.-Y. M. YOLOv4: Optimal speed and accuracy of object detection. arXiv preprint arXiv:2004.10934, 2020.

Boenm, B. W. A spiral model of software development and enhancement. Computer, vol. 21(5), pages 61-72, 1988.

Borowczyk, A., Nguyen, D.-T., Phu-Van Nguyen, A., Nguyen, D. Q., Saussié, D. and LE NY, J. Autonomous landing of a multirotor micro air vehicle on a high velocity ground vehicle. Ifac-Papersonline, vol. 50(1), pages 10488-10494, 2017.

Brockman, G., Cheung, V., Pettersion, L., Schneider, J., Schulman, J., Tang, J. and Zaremba, W. Openai gym. arXiv preprint arXiv:1606.01540, 2016.

Brown, T. B., Mann, B., Ryder, N., Subbiah, M., Kaplan, J., Dhariwal, P., Neelakantan, A., Shyam, P., Sastry, G., Askell, A. et Al. Language models are few-shot learners. arXiv preprint arXiv:2005.14165, 2020.

Caicedo, J. C. and Lazebnik, S. Active object localization with deep reinforcement learning. In Proceedings of the IEEE international conference on computer vision, pages 2488-2496. 2015.

Cantelli, L., Guastella, D., Melita, C., Muscato, G., Battiato, S., D’urso, F., Farinella, G., Ortis, A. and Santoro, C. Autonomous landing of a uav on a moving vehicle for the mbzirc. In Proceedings of 20th International Conference on Climbing and Walking Robots and the Support Technologies for Mobile Machines (CLAWAR). World Scientific, 2017.

Chen, X., Phang, S. K., Shan, M. and Chen, B. M. System integration of a visionguided uav for autonomous landing on moving platform. In 2016 12th IEEE International Conference on Control and Automation (ICCA), pages 761-766. IEEE, 2016. 
Chen, Y., Li, W., Sakaridis, C., Dai, D. and Van Gool, L. Domain adaptive faster $\mathrm{r}$-cnn for object detection in the wild. In Proceedings of the IEEE conference on computer vision and pattern recognition, pages 3339-3348. 2018.

Chor, H. and Kim, Y. Uav guidance using a monocular-vision sensor for aerial target tracking. Control Engineering Practice, vol. 22, pages 10-19, 2014.

Christnacher, F., Hengy, S., Laurenzis, M., Matwyschuk, A., Naz, P., Schertzer, S. and Schmitt, G. Optical and acoustical uav detection. In ElectroOptical Remote Sensing X, vol. 9988, page 99880B. International Society for Optics and Photonics, 2016.

Coumans, E. and BAI, Y. Pybullet, a python module for physics simulation for games, robotics and machine learning. 2016.

Dai, J., Li, Y., He, K. and Sun, J. R-fcn: Object detection via region-based fully convolutional networks. In Advances in neural information processing systems, pages 379-387. 2016.

Danelluan, M., Bhat, G., Shahbaz Khan, F. and Felsberg, M. Eco: Efficient convolution operators for tracking. In Proceedings of the IEEE conference on computer vision and pattern recognition, pages 6638-6646. 2017.

Delhaisse, B., Rozo, L. and Caldwell, D. G. Pyrobolearn: A python framework for robot learning practitioners. In Conference on Robot Learning, pages 1348-1358. 2020.

Deutsch, C., Meneghini, C., Mermut, O. and Lefort, M. Measuring technology readiness to improve innovation management. In Proc. of The XXI ISPIM Conference. 2010 .

Dosovitskiy, A. and DjolongA, J. You only train once: Loss-conditional training of deep networks. In International Conference on Learning Representations. 2019.

Ertel, W., Schneider, M., Cubek, R. and Tokic, M. The teaching-box: A universal robot learning framework. In 2009 International Conference on Advanced Robotics, pages 1-6. IEEE, 2009.

Falanga, D., Zanchettin, A., Simovic, A., Delmerico, J. and Scaramuzza, D. Vision-based autonomous quadrotor landing on a moving platform. In 2017 IEEE International Symposium on Safety, Security and Rescue Robotics (SSRR), pages 200-207. IEEE, 2017.

Fan, L., Zhu, Y., Zhu, J., Liu, Z., Zeng, O., Gupta, A., Creus-Costa, J., Savarese, S. and FeI-FeI, L. Surreal: Open-source reinforcement learning framework and robot manipulation benchmark. In Conference on Robot Learning, pages 767-782. 2018.

Feng, S., Whitman, E., Xinjilefu, X. and Atkeson, C. G. Optimization based full body control for the atlas robot. In 2014 IEEE-RAS International Conference on Humanoid Robots, pages 120-127. IEEE, 2014.

Ferigo, D., Traversaro, S., Metta, G. and Pucci, D. Gym-ignition: Reproducible robotic simulations for reinforcement learning. In 2020 IEEE/SICE International Symposium on System Integration (SII), pages 885-890. IEEE, 2020. 
Fernandez, R. A. S., Parrar, E. A., Milosevic, Z., Dominguez, S. and Rossi, C. Design, modeling and control of a spherical autonomous underwater vehicle for mine exploration. In 2018 IEEE/RSJ International Conference on Intelligent Robots and Systems (IROS), pages 1513-1519. IEEE, 2018.

Fukushima, K. Neocognitron: A hierarchical neural network capable of visual pattern recognition. Neural networks, vol. 1(2), pages 119-130, 1988.

Gao, M., Yu, R., Li, A., Morariu, V. I. and Davis, L. S. Dynamic zoom-in network for fast object detection in large images. In Proceedings of the IEEE Conference on Computer Vision and Pattern Recognition, pages 6926-6935. 2018.

Garrido-Jurado, S., Munoz-Salinas, R., Madrid-Cuevas, F. J. and MedinaCARnicer, R. Generation of fiducial marker dictionaries using mixed integer linear programming. Pattern Recognition, vol. 51, pages 481-491, 2016.

Gautam, A., Sujit, P. and Saripalli, S. Application of guidance laws to quadrotor landing. In 2015 International Conference on Unmanned Aircraft Systems (ICUAS), pages 372-379. IEEE, 2015.

GiRshick, R. Fast r-cnn. In Proceedings of the IEEE international conference on computer vision, pages 1440-1448. 2015.

Giusti, A., Guzzi, J., Cireşan, D. C., He, F.-L., Rodríguez, J. P., Fontana, F., Faessler, M., Forster, C., Schmidhuber, J., Di Caro, G. et Al. A machine learning approach to visual perception of forest trails for mobile robots. IEEE Robotics and Automation Letters, vol. 1(2), pages 661-667, 2015.

GökÇE, F., ÜÇOluk, G., ŞAhin, E. and Kalkan, S. Vision-based detection and distance estimation of micro unmanned aerial vehicles. Sensors, vol. 15(9), pages 23805-23846, 2015.

Goodfellow, I. J., Pouget-Abadie, J., Mirza, M., Xu, B., Warde-Farley, D., Ozair, S., Courville, A. and Bengio, Y. Generative adversarial networks. 2014.

Goodrich, M. A., Morse, B. S., Engh, C., Cooper, J. L. and Adams, J. A. Towards using unmanned aerial vehicles (uavs) in wilderness search and rescue: Lessons from field trials. Interaction Studies, vol. 10(3), pages 453-478, 2009.

Greatwood, C. and Richards, A. G. Reinforcement learning and model predictive control for robust embedded quadrotor guidance and control. Autonomous Robots, vol. 43(7), pages 1681-1693, 2019.

Gupta, A., Vedaldi, A. and Zisserman, A. Synthetic data for text localisation in natural images. In Proceedings of the IEEE conference on computer vision and pattern recognition, pages 2315-2324. 2016.

Hare, S., Golodetz, S., Saffari, A., Vineet, V., Cheng, M.-M., Hicks, S. L. and TorR, P. H. Struck: Structured output tracking with kernels. IEEE transactions on pattern analysis and machine intelligence, vol. 38(10), pages 2096-2109, 2015.

He, K., Gkioxari, G., Dollár, P. and Girshick, R. Mask r-cnn. In Proceedings of the IEEE international conference on computer vision, pages 2961-2969. 2017. 
He, K., Zhang, X., Ren, S. and Sun, J. Deep residual learning for image recognition. In Proceedings of the IEEE conference on computer vision and pattern recognition, pages 770-778. 2016.

Hebert, M. H., Thorpe, C. E. and Stentz, A. Intelligent unmanned ground vehicles: autonomous navigation research at Carnegie Mellon, vol. 388. Springer Science \& Business Media, 2012.

Hinterstoisser, S., Lepetit, V., Wohlhart, P. and Konolige, K. On pre-trained image features and synthetic images for deep learning. In Proceedings of the European Conference on Computer Vision (ECCV), pages 0-0. 2018.

Hinton, G. E. ET AL. Learning distributed representations of concepts. In Proceedings of the eighth annual conference of the cognitive science society, vol. 1, page 12. Amherst, MA, 1986.

Hoffmann, G., Waslander, S. and Tomlin, C. Quadrotor helicopter trajectory tracking control. In AIAA guidance, navigation and control conference and exhibit, page 7410 . 2008 .

Howard, A. G., Zhu, M., Chen, B., Kalenichenko, D., Wang, W., Weyand, T., Andreetto, M. and Adam, H. Mobilenets: Efficient convolutional neural networks for mobile vision applications. arXiv preprint arXiv:1704.04861, 2017.

Hu, B., Lu, L. and Mishra, S. Fast, safe and precise landing of a quadrotor on an oscillating platform. In 2015 American Control Conference (ACC), pages 3836-3841. IEEE, 2015.

Huang, R., Pedoeem, J. and Chen, C. Yolo-lite: a real-time object detection algorithm optimized for non-gpu computers. In 2018 IEEE International Conference on Big Data (Big Data), pages 2503-2510. IEEE, 2018.

Hutter, M., Gehring, C., Jud, D., Lauber, A., Bellicoso, C. D., Tsounis, V., Hwangbo, J., Bodie, K., Fankhauser, P., Bloesch, M. et Al. Anymal-a highly mobile and dynamic quadrupedal robot. In 2016 IEEE/RSJ International Conference on Intelligent Robots and Systems (IROS), pages 38-44. IEEE, 2016.

Hwangbo, J., Lee, J., Dosovitskiy, A., Bellicoso, D., Tsounis, V., Koltun, V. and Hutter, M. Learning agile and dynamic motor skills for legged robots. Science Robotics, vol. 4(26), 2019.

Hwangbo, J., Lee, J. and Hutter, M. Per-contact iteration method for solving contact dynamics. IEEE Robotics and Automation Letters, vol. 3(2), pages 895-902, 2018.

Hwangbo, J., Sa, I., Siegwart, R. and Hutter, M. Control of a quadrotor with reinforcement learning. IEEE Robotics and Automation Letters, vol. 2(4), pages 20962103, 2017.

James, S., Wohlhart, P., Kalakrishnan, M., Kalashnikov, D., Irpan, A., Ibarz, J., Levine, S., Hadsell, R. and Bousmalis, K. Sim-to-real via sim-to-sim: Dataefficient robotic grasping via randomized-to-canonical adaptation networks. In Proceedings of the IEEE Conference on Computer Vision and Pattern Recognition, pages 1262712637. 2019. 
Jie, Z., Liang, X., Feng, J., Jin, X., Lu, W. and Yan, S. Tree-structured reinforcement learning for sequential object localization. In Advances in Neural Information Processing Systems, pages 127-135. 2016.

Juliani, A., Berges, V.-P., Vekay, E., Gao, Y., Henry, H., Mattar, M. and LANGE, D. Unity: A general platform for intelligent agents. arXiv preprint arXiv:1809.02627, 2018.

Junell, J. L., Van Kampen, E.-J., De Visser, C. C. and Chu, Q. P. Reinforcement learning applied to a quadrotor guidance law in autonomous flight. In AIAA Guidance, Navigation, and Control Conference, page 1990. 2015.

Kai, W., Chunzhen, S. and Yi, J. Research on adaptive guidance technology of uav ship landing system based on net recovery. Procedia Engineering, vol. 99, pages 1027-1034, 2015 .

Kalal, Z., MikolajczyK, K. and Matas, J. Tracking-learning-detection. IEEE transactions on pattern analysis and machine intelligence, vol. 34(7), pages 1409-1422, 2011.

Kang, K., Belkhale, S., Kahn, G., Abbeel, P. and Levine, S. Generalization through simulation: Integrating simulated and real data into deep reinforcement learning for vision-based autonomous flight. CoRR, vol. abs/1902.03701, 2019a.

Kang, K., Belkhale, S., Kahn, G., Abbeel, P. and Levine, S. Generalization through simulation: Integrating simulated and real data into deep reinforcement learning for vision-based autonomous flight. In 2019 International Conference on Robotics and Automation (ICRA), pages 6008-6014. IEEE, 2019b.

Kim, D. K. and Chen, T. Deep neural network for real-time autonomous indoor navigation. arXiv preprint arXiv:1511.04668, 2015.

Kim, J., Jung, Y., LeE, D. and Shim, D. H. Landing control on a mobile platform for multi-copters using an omnidirectional image sensor. Journal of Intelligent $\mathcal{E}$ Robotic Systems, vol. 84(1-4), pages 529-541, 2016.

Kirtas, M., Tsampazis, K., Passalis, N. and Tefas, A. Deepbots: A webots-based deep reinforcement learning framework for robotics. In IFIP International Conference on Artificial Intelligence Applications and Innovations, pages 64-75. Springer, 2020.

Klein, G. and Murray, D. Parallel tracking and mapping for small AR workspaces. In Proc. Sixth IEEE and ACM International Symposium on Mixed and Augmented Reality (ISMAR'07). Nara, Japan, 2007.

Koch, W., Mancuso, R., West, R. and Bestavros, A. Reinforcement learning for uav attitude control. ACM Transactions on Cyber-Physical Systems, vol. 3(2), pages $1-21,2019$.

Kong, X., Xin, B., Wang, Y. and HuA, G. Collaborative deep reinforcement learning for joint object search. In Proceedings of the IEEE Conference on Computer Vision and Pattern Recognition, pages 1695-1704. 2017.

Kossaifi, J., Bulat, A., Tzimiropoulos, G. and Pantic, M. T-net: Parametrizing fully convolutional nets with a single high-order tensor. In Proceedings of the IEEE Conference on Computer Vision and Pattern Recognition, pages 7822-7831. 2019. 
Kothari, C. R. Research methodology: Methods and techniques. New Age International, 2004.

KoubÂA, A. and Qureshi, B. Dronetrack: Cloud-based real-time object tracking using unmanned aerial vehicles over the internet. IEEE Access, vol. 6, pages 13810-13824, 2018.

Krizhevsky, A., Sutskever, I. and Hinton, G. E. Imagenet classification with deep convolutional neural networks. In Advances in neural information processing systems, pages 1097-1105. 2012.

Lambert, N. O., Drew, D. S., Yaconelli, J., Levine, S., Calandra, R. and Pister, K. S. Low-level control of a quadrotor with deep model-based reinforcement learning. IEEE Robotics and Automation Letters, vol. 4(4), pages 4224-4230, 2019.

LeCun, Y., Bengio, Y. and Hinton, G. Deep learning. nature, vol. 521(7553), pages 436-444, 2015.

LeCun, Y., Boser, B., Denker, J. S., Henderson, D., Howard, R. E., Hubbard, W. and JACKEL, L. D. Backpropagation applied to handwritten zip code recognition. Neural computation, vol. 1(4), pages 541-551, 1989.

LeCun, Y., Bottou, L., Bengio, Y. and Haffner, P. Gradient-based learning applied to document recognition. Proceedings of the IEEE, vol. 86(11), pages 2278-2324, 1998.

LeE, D., Ryan, T. and Kim, H. J. Autonomous landing of a vtol uav on a moving platform using image-based visual servoing. In 2012 IEEE international conference on robotics and automation, pages 971-976. IEEE, 2012.

LeE, H., Jung, S. and Shim, D. H. Vision-based uav landing on the moving vehicle. In 2016 International conference on unmanned aircraft systems (ICUAS), pages 1-7. IEEE, 2016.

Li, J., Ye, D. H., Chung, T., Kolsch, M., Wachs, J. and Bouman, C. Multi-target detection and tracking from a single camera in unmanned aerial vehicles (uavs). In 2016 IEEE/RSJ International Conference on Intelligent Robots and Systems (IROS), pages 4992-4997. IEEE, 2016a.

Li, R., Pang, M., Zhao, C., Zhou, G. and Fang, L. Monocular long-term target following on uavs. In Proceedings of the IEEE Conference on Computer Vision and Pattern Recognition Workshops, pages 29-37. 2016b.

Li, S., Liu, T., Zhang, C., Yeung, D.-Y. and Shen, S. Learning unmanned aerial vehicle control for autonomous target following. arXiv preprint arXiv:1709.08233, 2017.

Liang, X., Lee, L. and XING, E. P. Deep variation-structured reinforcement learning for visual relationship and attribute detection. In Proceedings of the IEEE conference on computer vision and pattern recognition, pages 848-857. 2017.

Lillicrap, T. P., Hunt, J. J., Pritzel, A., Heess, N., Erez, T., Tassa, Y., Silver, D. and Wierstra, D. Continuous control with deep reinforcement learning. arXiv preprint arXiv:1509.02971, 2015. 
Lin, T.-Y., Goyal, P., Girshick, R., He, K. and Dollár, P. Focal loss for dense object detection. In Proceedings of the IEEE international conference on computer vision, pages 2980-2988. 2017.

Lin, T.-Y., Maire, M., Belongie, S., Hays, J., Perona, P., Ramanan, D., Dollár, P. and Zitnick, C. L. Microsoft coco: Common objects in context. In European conference on computer vision, pages 740-755. Springer, 2014.

Ling, K., Chow, D., Das, A. and Waslander, S. L. Autonomous maritime landings for low-cost vtol aerial vehicles. In 2014 Canadian Conference on Computer and Robot Vision, pages 32-39. IEEE, 2014.

LinnainmaA, S. The representation of the cumulative rounding error of an algorithm as a taylor expansion of the local rounding errors. Master's Thesis (in Finnish), Univ. Helsinki, pages 6-7, 1970.

Liu, M., Zhu, M., White, M., Li, Y. and Kalenichenko, D. Looking fast and slow: Memory-guided mobile video object detection. arXiv preprint arXiv:1903.10172, 2019a.

Liu, P., Chen, A. Y., Huang, Y.-N., Han, J.-Y., Lai, J.-S., Kang, S.-C., Wu, T.H., Wen, M.-C., Tsai, M.-H. ET AL. A review of rotorcraft unmanned aerial vehicle (uav) developments and applications in civil engineering. Smart Structures and Systems, vol. 13(6), pages 1065-1094, 2014.

Liu, S., HuAng, D. and WAng, Y. Learning spatial fusion for single-shot object detection. arXiv preprint arXiv:1911.09516, 2019b.

Liu, W., Anguelov, D., Erhan, D., Szegedy, C., Reed, S., Fu, C.-Y. and Berg, A. C. Ssd: Single shot multibox detector. In European conference on computer vision, pages 21-37. Springer, 2016a.

Liu, W., Liu, J. and Luo, B. Can synthetic data improve object detection results for remote sensing images? arXiv preprint arXiv:2006.05015, 2020.

Liu, X., Liang, W., Wang, Y., Li, S. and Pei, M. 3d head pose estimation with convolutional neural network trained on synthetic images. In 2016 IEEE International Conference on Image Processing (ICIP), pages 1289-1293. IEEE, 2016b.

Long, J., Shelhamer, E. and Darrell, T. Fully convolutional networks for semantic segmentation. In Proceedings of the IEEE conference on computer vision and pattern recognition, pages 3431-3440. 2015.

Loquercio, A., Maqueda, A. I., Del-Blanco, C. R. and Scaramuzza, D. Dronet: Learning to fly by driving. IEEE Robotics and Automation Letters, vol. 3(2), pages 1088-1095, 2018.

Luo, H., Xie, W., Wang, X. and Zeng, W. Detect or track: Towards cost-effective video object detection/tracking. In Proceedings of the AAAI Conference on Artificial Intelligence, vol. 33, pages 8803-8810. 2019.

Macés-Hernández, J. A., Defaÿ, F. and Chauffaut, C. Autonomous landing of an uav on a moving platform using model predictive control. In 2017 11th Asian Control Conference (ASCC), pages 2298-2303. IEEE, 2017. 
Mahasseni, B., Todorovic, S. and Fern, A. Budget-aware deep semantic video segmentation. In Proceedings of the IEEE Conference on Computer Vision and Pattern Recognition, pages 1029-1038. 2017.

MaO, H., Yao, S., Tang, T., Li, B., Yao, J. and Wang, Y. Towards real-time object detection on embedded systems. IEEE Transactions on Emerging Topics in Computing, vol. 6(3), pages 417-431, 2016.

Marani, G., Choi, S. K. and Yuh, J. Underwater autonomous manipulation for intervention missions auvs. Ocean Engineering, vol. 36(1), pages 15-23, 2009.

Mathe, S., Pirinen, A. and Sminchisescu, C. Reinforcement learning for visual object detection. In Proceedings of the IEEE Conference on Computer Vision and Pattern Recognition, pages 2894-2902. 2016.

Matthies, L., Kelly, A., Litwin, T. and Tharp, G. Obstacle detection for unmanned ground vehicles: A progress report. In Robotics Research, pages 475-486. Springer, 1996.

McCarthy, J., Minsky, M. L., Rochester, N. and Shannon, C. E. A proposal for the dartmouth summer research project on artificial intelligence, august 31, 1955. AI magazine, vol. 27(4), pages 12-12, 2006.

McCulloch, W. S. and Pitts, W. A logical calculus of the ideas immanent in nervous activity. The bulletin of mathematical biophysics, vol. 5(4), pages 115-133, 1943.

MCNeEla, D. The reinforce algorithm aka monte-carlo policy differentiation. 2019.

Meier, L., Tanskanen, P., Fraundorfer, F. and Pollefeys, M. Pixhawk: A system for autonomous flight using onboard computer vision. In 2011 IEEE International Conference on Robotics and Automation, pages 2992-2997. IEEE, 2011.

Michael, N., Mellinger, D., Lindsey, Q. and Kumar, V. The grasp multiple microuav testbed. IEEE Robotics \& Automation Magazine, vol. 17(3), pages 56-65, 2010.

Michel, O. Cyberbotics ltd. webots: professional mobile robot simulation. International Journal of Advanced Robotic Systems, vol. 1(1), page 5, 2004.

Mnih, V., Kavukcuoglu, K., Silver, D., Graves, A., Antonoglou, I., Wierstra, D. and Riedmiller, M. Playing atari with deep reinforcement learning. arXiv preprint arXiv:1312.5602, 2013.

Mnih, V., Kavukcuoglu, K., Silver, D., Rusu, A. A., Veness, J., Bellemare, M. G., Graves, A., Riedmiller, M., Fidjeland, A. K., Ostrovski, G. et al. Human-level control through deep reinforcement learning. nature, vol. 518(7540), pages $529-533,2015$.

Molchanov, A., Chen, T., Hönig, W., Preiss, J. A., Ayanian, N. and Sukhatme, G. S. Sim-to-(multi)-real: Transfer of low-level robust control policies to multiple quadrotors. arXiv preprint arXiv:1903.04628, 2019.

Mondragón, I. F., Campoy, P., Olivares-Mendez, M. A. and Martinez, C. 3d object following based on visual information for unmanned aerial vehicles. In IX Latin American Robotics Symposium and IEEE Colombian Conference on Automatic Control, 2011 IEEE, pages 1-7. IEEE, 2011. 
Mou, X. Artificial intelligence: Investment trends and selected industry uses. International Finance Corporation, pages 1-8, 2019.

Mueller, M., Sharma, G., Smith, N. and Ghanem, B. Persistent aerial tracking system for uavs. In 2016 IEEE/RSJ International Conference on Intelligent Robots and Systems (IROS), pages 1562-1569. IEEE, 2016.

Nagabandi, A., Konolige, K., Levine, S. and Kumar, V. Deep dynamics models for learning dexterous manipulation. In Conference on Robot Learning, pages 1101-1112. 2020 .

Nägeli, T., Oberholzer, S., Plüss, S., Alonso-Mora, J. and Hilliges, O. Flycon: real-time environment-independent multi-view human pose estimation with aerial vehicles. ACM Transactions on Graphics (TOG), vol. 37(6), pages 1-14, 2018.

Nebehay, G. and Pflugfelder, R. Consensus-based matching and tracking of keypoints for object tracking. In IEEE Winter Conference on Applications of Computer Vision, pages 862-869. IEEE, 2014.

Nissen, S. ET AL. Implementation of a fast artificial neural network library (fann). Report, Department of Computer Science University of Copenhagen (DIKU), vol. 31, page 29, 2003.

Nuin, Y. L. E., Lopez, N. G., Moral, E. B., Juan, L. U. S., Rueda, A. S., Vilches, V. M. and KoJCEv, R. Ros2learn: a reinforcement learning framework for ros 2. arXiv preprint arXiv:1903.06282, 2019.

Olivares-Mendez, M. A., Mondragon, I., Campoy Cervera, P., Mejias, L. and Martinez, C. Aerial object following using visual fuzzy servoing. In Proceedings of the 1st Workshop on Research, Development and Education on Unmanned Aerial Systems (RED-UAS 2011), pages 61-70. Centro Avanzado de Tecnologías Aeroespaciales (CATEC), 2011.

Opromolla, R., Fasano, G. and Accardo, D. A vision-based approach to uav detection and tracking in cooperative applications. Sensors, vol. 18(10), page 3391, 2018.

Pellissier, R. Business research made easy. Juta and Company Ltd, 2008.

Peng, X., Sun, B., Ali, K. and Saenko, K. Learning deep object detectors from 3d models. In Proceedings of the IEEE International Conference on Computer Vision, pages 1278-1286. 2015.

PÉREZ, C. S. Learning-based perception, control, and navigation for autonomous missions in aerial robotics, 2019.

Pestana, J., Sanchez-Lopez, J. L., Saripalli, S. and Campoy, P. Computer vision based general object following for gps-denied multirotor unmanned vehicles. In 2014 American Control Conference, pages 1886-1891. IEEE, 2014.

Pi, C.-H., Hu, K.-C., Cheng, S. and Wu, I.-C. Low-level autonomous control and tracking of quadrotor using reinforcement learning. Control Engineering Practice, vol. 95, page 104222, 2020. 
Pirinen, A. and Sminchisescu, C. Deep reinforcement learning of region proposal networks for object detection. In Proceedings of the IEEE Conference on Computer Vision and Pattern Recognition, pages 6945-6954. 2018.

Quigley, M., Conley, K., Gerkey, B., Faust, J., Foote, T., Leibs, J., Wheeler, R. and NG, A. Y. Ros: an open-source robot operating system. In ICRA workshop on open source software, vol. 3, page 5. Kobe, Japan, 2009.

Rabelo, M. F. S., Brandao, A. S. and Sarcinelli-Filho, M. Landing a uav on static or moving platforms using a formation controller. IEEE Systems Journal, 2020.

Rad, M., Oberweger, M. and Lepetit, V. Feature mapping for learning fast and accurate $3 \mathrm{~d}$ pose inference from synthetic images. In Proceedings of the IEEE Conference on Computer Vision and Pattern Recognition, pages 4663-4672. 2018.

Rajpura, P. S., Bojinov, H. and Hegde, R. S. Object detection using deep cnns trained on synthetic images. arXiv preprint arXiv:1706.06782, 2017.

Rao, K., Harris, C., Irpan, A., Levine, S., Ibarz, J. and Khansari, M. Rl-cyclegan: Reinforcement learning aware simulation-to-real. In Proceedings of the IEEE/CVF Conference on Computer Vision and Pattern Recognition, pages 11157-11166. 2020.

Redmon, J. and Farhadi, A. Yolo9000: better, faster, stronger. In Proceedings of the IEEE conference on computer vision and pattern recognition, pages 7263-7271. 2017.

Redmon, J. and FARHAdi, A. Yolov3: An incremental improvement. arXiv, 2018.

Ren, S., He, K., Girshick, R. and Sun, J. Faster r-cnn: Towards real-time object detection with region proposal networks. In Advances in neural information processing systems, pages 91-99. 2015.

Rodriguez-Ramos, A., Sampedro, C., Bavle, H., Milosevic, Z., GarciaVAquero, A. and CAMPOY, P. Towards fully autonomous landing on moving platforms for rotary unmanned aerial vehicles. In 2017 International Conference on Unmanned Aircraft Systems (ICUAS), pages 170-178. IEEE, 2017.

Rodriguez-Ramos, A., Sampedro, C., Bavle, H., Moreno, I. G. and Campoy, P. A deep reinforcement learning technique for vision-based autonomous multirotor landing on a moving platform. In 2018 IEEE/RSJ International Conference on Intelligent Robots and Systems (IROS), pages 1010-1017. IEEE, 2018.

Romero-Ramirez, F. J., Muñoz-Salinas, R. and Medina-Carnicer, R. Speeded up detection of squared fiducial markers. Image and vision Computing, vol. 76, pages $38-47,2018$.

Ros, G., Sellart, L., Materzynska, J., Vazquez, D. and Lopez, A. M. The synthia dataset: A large collection of synthetic images for semantic segmentation of urban scenes. In Proceedings of the IEEE conference on computer vision and pattern recognition, pages 3234-3243. 2016.

Rozantsev, A. Vision-based detection of aircrafts and uavs. Technical report, EPFL, 2017. 
Rozantsev, A., Lepetit, V. and Fua, P. Flying objects detection from a single moving camera. In Proceedings of the IEEE Conference on Computer Vision and Pattern Recognition, pages 4128-4136. 2015a.

Rozantsev, A., Lepetit, V. and Fua, P. On rendering synthetic images for training an object detector. Computer Vision and Image Understanding, vol. 137, pages 24-37, 2015 b.

Rozantsev, A., Lepetit, V. and Fua, P. Detecting flying objects using a single moving camera. IEEE transactions on pattern analysis and machine intelligence, vol. 39(5), pages 879-892, 2016.

Rucco, A., Sujit, P., Aguiar, A. P., De Sousa, J. B. and Pereira, F. L. Optimal rendezvous trajectory for unmanned aerial-ground vehicles. IEEE Transactions on Aerospace and Electronic Systems, vol. 54(2), pages 834-847, 2017.

Rungsuptaweekoon, K., Visoottiviseth, V. and Takano, R. Evaluating the power efficiency of deep learning inference on embedded gpu systems. In 2017 2nd International Conference on Information Technology (INCIT), pages 1-5. IEEE, 2017.

Russakovsky, O., Deng, J., Su, H., Krause, J., Satheesh, S., Ma, S., Huang, Z., Karpathy, A., Khosla, A., Bernstein, M., Berg, A. C. and Fei-Fei, L. ImageNet Large Scale Visual Recognition Challenge. International Journal of Computer Vision (IJCV), vol. 115(3), pages 211-252, 2015.

Sadeghi, F. and Levine, S. Cad2rl: Real single-image flight without a single real image. arXiv preprint arXiv:1611.04201, 2016.

Sadin, S. R., Povinelli, F. P. and Rosen, R. The nasa technology push towards future space mission systems. In Space and Humanity, pages 73-77. Elsevier, 1989.

Salakhutdinov, R. and Hinton, G. Deep boltzmann machines. In Artificial intelligence and statistics, pages 448-455. 2009.

Sampedro, C., Bavle, H., Rodriguez-Ramos, A., de la Puente, P. and Campoy, $\mathrm{P}$. Laser-based reactive navigation for multirotor aerial robots using deep reinforcement learning. In 2018 IEEE/RSJ International Conference on Intelligent Robots and Systems (IROS), pages 1024-1031. IEEE, 2018a.

Sampedro, C., Rodriguez-Ramos, A., Bavle, H., Carrio, A., de la Puente, P. and CAMPoY, P. A fully-autonomous aerial robot for search and rescue applications in indoor environments using learning-based techniques. Journal of Intelligent 63 Robotic Systems, vol. 95(2), pages 601-627, 2019.

Sampedro, C., Rodriguez-Ramos, A., Gil, I., Mejias, L. and Campoy, P. Imagebased visual servoing controller for multirotor aerial robots using deep reinforcement learning. In 2018 IEEE/RSJ International Conference on Intelligent Robots and Systems (IROS), pages 979-986. IEEE, 2018b.

Sanchez-lopez, J. L., Fernández, R. A. S., Bavle, H., Sampedro, C., Molina, M., Pestana, J. and Campoy, P. Aerostack: An architecture and open-source software framework for aerial robotics. In 2016 International Conference on Unmanned Aircraft Systems (ICUAS), pages 332-341. IEEE, 2016. 
Sandler, M., Howard, A., Zhu, M., Zhmoginov, A. and Chen, L.-C. Mobilenetv2: Inverted residuals and linear bottlenecks. In Proceedings of the IEEE conference on computer vision and pattern recognition, pages 4510-4520. 2018.

Schulman, J., Levine, S., Abbeel, P., Jordan, M. and Moritz, P. Trust region policy optimization. In International conference on machine learning, pages 1889-1897. 2015 .

Schulman, J., Wolski, F., Dhariwal, P., Radford, A. and Klimov, O. Proximal policy optimization algorithms. arXiv preprint arXiv:1707.0634\%, 2017.

Schumann, A., Sommer, L., Klatte, J., Schuchert, T. and Beyerer, J. Deep cross-domain flying object classification for robust uav detection. In 2017 14th IEEE International Conference on Advanced Video and Signal Based Surveillance (AVSS), pages 1-6. IEEE, 2017.

Shah, S., Dey, D., Lovett, C. and Kapoor, A. Airsim: High-fidelity visual and physical simulation for autonomous vehicles. In Field and service robotics, pages 621635. Springer, 2018.

Shaker, M., Smith, M. N., Yue, S. and Duckett, T. Vision-based landing of a simulated unmanned aerial vehicle with fast reinforcement learning. In 2010 International Conference on Emerging Security Technologies, pages 183-188. IEEE, 2010.

Shimoda, S., Kuroda, Y. and Ingnemma, K. Potential field navigation of high speed unmanned ground vehicles on uneven terrain. In Proceedings of the 2005 IEEE International Conference on Robotics and Automation, pages 2828-2833. IEEE, 2005.

Shrivastava, A., Pfister, T., Tuzel, O., Susskind, J., Wang, W. and Webb, R. Learning from simulated and unsupervised images through adversarial training. In Proceedings of the IEEE conference on computer vision and pattern recognition, pages 21072116. 2017.

Silver, D., Lever, G., Heess, N., Degris, T., Wierstra, D. and Riedmiller, M. Deterministic policy gradient algorithms. 2014.

Simonyan, K. and Zisserman, A. Very deep convolutional networks for large-scale image recognition. arXiv preprint arXiv:1409.1556, 2014.

Singla, A., Padakandla, S. and Bhatnagar, S. Memory-based deep reinforcement learning for obstacle avoidance in uav with limited environment knowledge. IEEE Transactions on Intelligent Transportation Systems, 2019.

Supancic III, J. and Ramanan, D. Tracking as online decision-making: Learning a policy from streaming videos with reinforcement learning. In Proceedings of the IEEE International Conference on Computer Vision, pages 322-331. 2017.

Sutton, R. S. and Barto, A. G. Reinforcement learning: An introduction. MIT press, 2018.

Szegedy, C., Liu, W., Jia, Y., Sermanet, P., Reed, S., Anguelov, D., Erhan, D., Vanhoucke, V. and Rabinovich, A. Going deeper with convolutions. In Proceedings of the IEEE conference on computer vision and pattern recognition, pages 1-9. 2015. 
Szegedy, C., Vanhoucke, V., Ioffe, S., Shlens, J. and Wojna, Z. Rethinking the inception architecture for computer vision. In Proceedings of the IEEE conference on computer vision and pattern recognition, pages 2818-2826. 2016.

Tan, J., Zhang, T., Coumans, E., Iscen, A., Bai, Y., Hafner, D., Bohez, S. and VANHOUCKE, V. Sim-to-real: Learning agile locomotion for quadruped robots. arXiv preprint arXiv:1804.10332, 2018.

Tan, M., Pang, R. and Le, Q. V. Efficientdet: Scalable and efficient object detection. arXiv preprint arXiv:1911.09070, 2019.

Teuliere, C., Eck, L. and Marchand, E. Chasing a moving target from a flying uav. In 2011 IEEE/RSJ International Conference on Intelligent Robots and Systems, pages 4929-4934. IEEE, 2011.

Tobin, J., Fong, R., Ray, A., Schneider, J., Zaremba, W. and Abbeel, P. Domain randomization for transferring deep neural networks from simulation to the real world. In 2017 IEEE/RSJ International Conference on Intelligent Robots and Systems (IROS), pages 23-30. IEEE, 2017.

Tremblay, J., Prakash, A., Acuna, D., Brophy, M., Jampani, V., Anil, C., To, T., Cameracci, E., Boochoon, S. and Birchfield, S. Training deep networks with synthetic data: Bridging the reality gap by domain randomization. In Proceedings of the IEEE Conference on Computer Vision and Pattern Recognition Workshops, pages 969-977. 2018.

Uhlenbeck, G. E. and Ornstein, L. S. On the theory of the brownian motion. Physical review, vol. 36(5), page 823, 1930.

Unlu, E., Zenou, E. and Riviere, N. Using shape descriptors for uav detection. Electronic Imaging, vol. 2018(9), pages 128-1, 2018.

Uzkent, B. and ERmon, S. Learning when and where to zoom with deep reinforcement learning. In Proceedings of the IEEE/CVF Conference on Computer Vision and Pattern Recognition, pages 12345-12354. 2020.

Uzkent, B., Yeh, C. and Ermon, S. Efficient object detection in large images using deep reinforcement learning. In The IEEE Winter Conference on Applications of Computer Vision, pages 1824-1833. 2020.

Valavanis, K. P. and Vachtsevanos, G. J. Handbook of unmanned aerial vehicles, vol. 1. Springer, 2015.

Vankadari, M. B., Das, K., Shinde, C. and Kumar, S. A reinforcement learning approach for autonomous control and landing of a quadrotor. In 2018 International Conference on Unmanned Aircraft Systems (ICUAS), pages 676-683. IEEE, 2018.

Vassilo, K., Heatwole, C., Taha, T. and Mehmood, A. Multi-step reinforcement learning for single image super-resolution. In Proceedings of the IEEE/CVF Conference on Computer Vision and Pattern Recognition Workshops, pages 512-513. 2020.

Vaswani, A., Shazeer, N., Parmar, N., Uszkoreit, J., Jones, L., Gomez, A. N., Kaiser, Ł. and Polosukhin, I. Attention is all you need. In Advances in neural information processing systems, pages 5998-6008. 2017. 
Vlantis, P., Marantos, P., Bechlioulis, C. P. and Kyriakopoulos, K. J. Quadrotor landing on an inclined platform of a moving ground vehicle. In 2015 IEEE International Conference on Robotics and Automation (ICRA), pages 2202-2207. IEEE, 2015.

VRba, M., Heřt, D. and SAska, M. Onboard marker-less detection and localization of non-cooperating drones for their safe interception by an autonomous aerial system. IEEE Robotics and Automation Letters, vol. 4(4), pages 3402-3409, 2019.

Wang, C., Wang, J., Wang, J. and Zhang, X. Deep reinforcement learning-based autonomous uav navigation with sparse rewards. IEEE Internet of Things Journal, 2020 .

Wang, F., Zhou, B., Chen, K., Fan, T., Zhang, X., Li, J., Tian, H. and Pan, J. Intervention aided reinforcement learning for safe and practical policy optimization in navigation. arXiv preprint arXiv:1811.06187, 2018.

Wang, Z., Bapst, V., Heess, N., Mnih, V., Munos, R., Kavukcuoglu, K. and DE Freitas, N. Sample efficient actor-critic with experience replay. arXiv preprint arXiv:1611.01224, 2016.

Wenzel, K. E., Masselli, A. and Zell, A. Automatic take off, tracking and landing of a miniature uav on a moving carrier vehicle. Journal of intelligent $\mathcal{G}$ robotic systems, vol. 61(1-4), pages 221-238, 2011.

Wu, T.-C., Tseng, S.-Y., LAI, C.-F., Ho, C.-Y. and LAI, Y.-H. Navigating assistance system for quadcopter with deep reinforcement learning. In 2018 1st International Cognitive Cities Conference (IC3), pages 16-19. IEEE, 2018.

Wynn, R. B., Huvenne, V. A., le Bas, T. P., Murton, B. J., Connelly, D. P., Bett, B. J., Ruhl, H. A., Morris, K. J., Peakall, J., Parsons, D. R. et al. Autonomous underwater vehicles (auvs): Their past, present and future contributions to the advancement of marine geoscience. Marine Geology, vol. 352, pages 451-468, 2014.

Xia, F., Zamir, A. R., He, Z., Sax, A., Malik, J. and Savarese, S. Gibson env: Real-world perception for embodied agents. In Proceedings of the IEEE Conference on Computer Vision and Pattern Recognition, pages 9068-9079. 2018.

Yao, N., Anaya, E., Tao, Q., Cho, S., Zheng, H. and Zhang, F. Monocular visionbased human following on miniature robotic blimp. In 2017 IEEE International Conference on Robotics and Automation (ICRA), pages 3244-3249. IEEE, 2017.

Ye, D. H., Li, J., Chen, Q., Wachs, J. and Bouman, C. Deep learning for moving object detection and tracking from a single camera in unmanned aerial vehicles (uavs). Electronic Imaging, vol. 2018(10), pages 466-1, 2018.

Yu, J., Guo, K., Hu, Y., Ning, X., Qiu, J., Mao, H., Yao, S., Tang, T., Li, B., WANG, Y. ET AL. Real-time object detection towards high power efficiency. In 2018 Design, Automation \&S Test in Europe Conference $\mathcal{G}$ Exhibition (DATE), pages 704-708. IEEE, 2018.

ZAFrA, J. F. Sistema uav de seguimiento visual basado en aprendizaje por refuerzo y aleatorización de dominio, 2020. 
Zamora, I., Lopez, N. G., Vilches, V. M. and Cordero, A. H. Extending the openai gym for robotics: a toolkit for reinforcement learning using ros and gazebo. arXiv preprint arXiv:1608.05742, 2016.

Zhang, J., Tai, L., Yun, P., Xiong, Y., Liu, M., Boedecker, J. and Burgard, W. Vr-goggles for robots: Real-to-sim domain adaptation for visual control. IEEE Robotics and Automation Letters, vol. 4(2), pages 1148-1155, 2019.

Zhang, Q.-X., Lin, G.-H., Zhang, Y.-M., Xu, G. and Wang, J.-J. Wildland forest fire smoke detection based on faster $\mathrm{r}$-cnn using synthetic smoke images. Procedia engineering, vol. 211, pages 441-446, 2018.

Zhang, T., Kahn, G., Levine, S. and Abbeel, P. Learning deep control policies for autonomous aerial vehicles with mpc-guided policy search. In 2016 IEEE international conference on robotics and automation (ICRA), pages 528-535. IEEE, 2016.

Zhao, W., Zhang, S., Guan, Z., Zhao, W., Peng, J. and Fan, J. Learning deep network for detecting $3 \mathrm{~d}$ object keypoints and $6 \mathrm{~d}$ poses. In Proceedings of the IEEE/CVF Conference on Computer Vision and Pattern Recognition, pages 14134-14142. 2020.

Zhou, X., Wang, D. and KrÄhenbÜHL, P. Objects as points. arXiv preprint arXiv:1904.07850, 2019.

Zhu, J.-Y., PArk, T., Isola, P. and Efros, A. A. Unpaired image-to-image translation using cycle-consistent adversarial networks. In Proceedings of the IEEE international conference on computer vision, pages 2223-2232. 2017. 